\title{
Burst Spinal Cord Stimulation in a Rat Model of Chronic Neuropathic pain
}

Citation for published version (APA):

Meuwissen, K. (2019). Burst Spinal Cord Stimulation in a Rat Model of Chronic Neuropathic pain: Spinal and Supraspinal Mechanisms. [Doctoral Thesis, Maastricht University]. Gildeprint Drukkerijen. https://doi.org/10.26481/dis.20191205kw

Document status and date:

Published: 01/01/2019

DOI:

$10.26481 /$ dis.20191205kw

Document Version:

Publisher's PDF, also known as Version of record

\section{Please check the document version of this publication:}

- A submitted manuscript is the version of the article upon submission and before peer-review. There can be important differences between the submitted version and the official published version of record.

People interested in the research are advised to contact the author for the final version of the publication, or visit the DOI to the publisher's website.

- The final author version and the galley proof are versions of the publication after peer review.

- The final published version features the final layout of the paper including the volume, issue and page numbers.

Link to publication

\footnotetext{
General rights rights.

- You may freely distribute the URL identifying the publication in the public portal. please follow below link for the End User Agreement:

www.umlib.nl/taverne-license

Take down policy

If you believe that this document breaches copyright please contact us at:

repository@maastrichtuniversity.nl

providing details and we will investigate your claim.
}

Copyright and moral rights for the publications made accessible in the public portal are retained by the authors and/or other copyright owners and it is a condition of accessing publications that users recognise and abide by the legal requirements associated with these

- Users may download and print one copy of any publication from the public portal for the purpose of private study or research.

- You may not further distribute the material or use it for any profit-making activity or commercial gain

If the publication is distributed under the terms of Article $25 \mathrm{fa}$ of the Dutch Copyright Act, indicated by the "Taverne" license above, 
(C) 2019 Koen Meuwissen, Maastricht 2019

\section{Cover design Koen Meuwissen}

ISBN 978-94-6323-760-4

Printed by: Gildeprint

All rights reserved. No part of this publication may be produced or transmitted in any form or byany means, electronic or mechanical, including photocopy, recording or otherwise, without written permission of the author. 
Burst Spinal Cord Stimulation in a Rat Model of Chronic Neuropathic Pain: Spinal and Supraspinal Mechanisms.

\section{PROEFSCHRIFT}

Ter verkrijging van de graad van doctor aan de Universiteit Maastricht, op gezag van de Rector Magnificus, Prof. dr. Rianne M. Letschert volgens het besluit van het College van Decanen in het openbaar te verdedigen op donderdag 5 december 2019 om 10:00 uur

$$
\text { door }
$$

Koen P.V. Meuwissen 


\section{Promotoren:}

Prof. Dr. E.A.J. Joosten

Prof. Dr. M. van Kleef

\section{Beoordelingscommissie:}

Prof. Dr. J. Prickaerts (voorzitter)

Prof. Dr. G. van Koeveringe

Prof. Dr. B. Linderoth (Karolinska Institutet, Sweden)

Prof. Dr. Y. Temel

Prof. Dr. K. Vissers (Radboud UMC, Nijmegen)

Financial support for the completion of this thesis was provided in the form of a research grant from:

\section{Scientific}




\section{Table of Contents}

Chapter 1

Chapter 2

Chapter 3

Chapter 4

Chapter 5

Burst and Tonic Spinal Cord Stimulation in the Mechanical

Conflict-Avoidance System: Cognitive-Motivational Aspects

Accepted. Meuwissen et al. Neuromodulation: Technology at the Neural Interface. 2019

Chapter 6

The Supraspinal Mechanisms of Burst Spinal Cord Stimulation: a Functional Magnetic Resonance Study in Peripherally Injured Chronic Neuropathic Rats

Submitted

Chapter 7

Summary, discussion, and conclusion

Valorization

Curriculum Vitae

List of publications

Acknowledgements 


\section{Preface}

Neuropathic pain is pain that arises as a direct consequence of a lesion or diseases affecting the somatosensory system [1]. Despite the abundant availability of drugs and guidelines for the treatment of neuropathic pain, it remains a disorder often under- or untreated. Over the past 5 decades, spinal cord stimulation (SCS) has become a safe and effective, lastresort, therapeutic tool for the treatment of intractable neuropathic pain conditions. Literature shows that Tonic (or conventional) SCS is clinically effective for the management of failed back surgery syndrome (FBSS) and complex regional pain syndrome (CRPS); two neuropathic pain disorders infamous for their resistance to pharmacological treatment. Although effective, roughly $50-70 \%$ of patients implanted with a SCS-device experiences adequate pain relief, there still remains a need for improvement, as $30-50 \%$ of patients do not achieve adequate pain relief and/or experiences loss of efficacy over time. Unfortunately, the reported success rate of Tonic SCS in treatment of FBSS and CRPS has improved little over the past decade and SCS remains an indispensable, though suboptimal, treatment method for chronic neuropathic pain. Additionally, the presence of paresthesia, a tingling sensation experienced in the stimulated limb or area with Tonic SCS, has hindered the design of double-blind placebo-controlled study designs.

In an effort to improve the efficacy of SCS, several alternative stimulation paradigms have been developed over the last decade. One such example is the Burst paradigm, a SCSmode that uses periodic bursts of pulses rather than continuous pulses as seen with Tonic SCS. Burst stimulation is paresthesia-free and suggested to induce additional pain relief over Tonic stimulation. Literature suggests that the distinct analgesic effect of Burst SCS is due to the modulation of cognitive-emotional aspects of pain. Initial studies on Burst SCS indicate great potential for this novel stimulation paradigm, yet, also address a need for further optimization. To this end, experimental studies should be performed in order to acquire more Insight into the underlying mechanism and, along the way, uncover the full potential of Burst stimulation in the treatment of neuropathic pain. 
CHAPTER 1 GENERAL INTRODUCTION

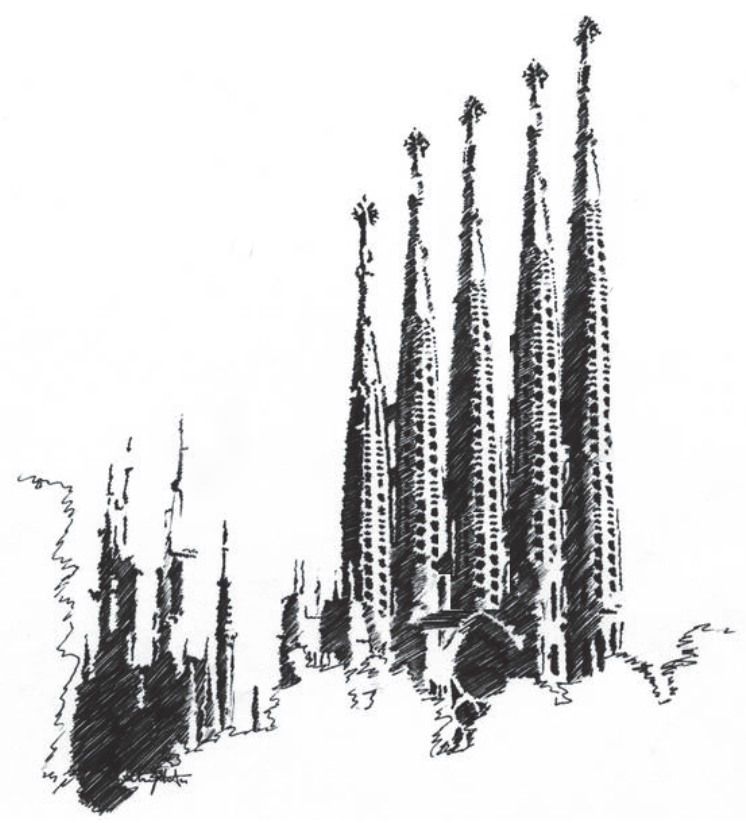


In view of the physiological and neurological aspects involved in neuropathic pain, this chapter will firstly summarize the pathophysiological and epidemiological characteristics. (1.1) This is followed by a short summary on two intractable neuropathic pain disorders frequently treated with spinal cord stimulation (SCS) (1.2). Next, treatment of neuropathic pain with Tonic SCS will be described (1.3). Novel SCS paradigms will be introduced (1.4). Clinical and experimental findings on Burst SCS will be discussed (1.5). Lastly, the aims, outline, and research questions of this academic thesis will be presented (1.6).

\subsection{Neuropathic pain and pathophysiology}

Neuropathic pain (NPP) has been described as 'the most terrible of all tortures, which a nerve wound may inflict' [2]. NPP is characterized by sensory abnormalities such as abnormal unpleasant sensation (dysesthesia), an increased sensitivity to painful stimuli (hyperalgesia) and pain in response to normally innocuous stimuli (allodynia) [3, 4]. Over the years, various definitions of NPP have been used. Yet, the most recent and widely accepted definition is stated by the International Association for the Study of Pain (IASP) [5]:

"Pain caused by a lesion or diseases of the somatosensory system".

The somatosensory system allows for the perception of touch, pressure, pain, temperature, movement and vibration. Nerves endings from the somatosensory nerves arise in the skin, muscles, joints and fascia and include nociceptors, mechanoreceptors and chemoreceptors. When stimulated, these receptors send signals to the spinal cord and eventually to the brain for further processing. Lesions or diseases that affect the somatosensory nervous system can lead to altered or disordered transmission to the central nervous system. Distorted signal transmission and processing can, if not treated promptly, lead to the development of NPP. At that point, the distorted processing of somatosensory signals goes beyond normal plasticity and enters an aberrant state. Nerve lesions or diseases that lead to the development of NPP can have various etiologies, such as infection, peripheral nerve injury, nerve compression, failed back surgery, diabetic neuropathy, chemotherapy, and amputation. Based on the location of the injury or disease, in the peripheral of central nervous system, NPP is classified as either peripheral NPP or central NPP. However, it is likely that at a progressed, chronic, state NPP involves both peripheral and central mechanisms. Importantly, NPP is seen as mechanistically dissimilar to other chronic pain 
conditions such as inflammatory pain where the primary cause is inflammation with altered chemical signaling at the site of inflammation [6].

Patients diagnosed with NPP typically experience a distinct set of symptoms, such as burning, stabbing or electric shock-like sensations [7]. These experiences can either be of a spontaneous nature or triggered by an external stimulus. Stimulus-evoked pain can be classified as pain in response to non-painful stimuli: allodynia, or as an increased response to painful stimuli: hyperalgesia. Generally, NPP-patients experience both allodynia and hyperalgesia together. The aforementioned collection of symptoms makes that NPP is associated with a significant decrease in patients' health related quality of life (HRQol) [8, 9]. Furthermore, studies on NPP-patient populations report an increase in drug prescriptions and visits to health care providers $[8,10]$. Sleep disturbances, anxiety and depression are frequent comorbidities in patients with NPP, and quality of life is more impaired in patients with chronic neuropathic pain than in those with chronic nonneuropathic pain that does not originate from damaged or irritated nerves [3].

\subsubsection{Epidemiology of neuropathic pain}

Making an adequate estimation of the incidence and prevalence of NPP has always been difficult due to the lack of simple diagnostic criteria for large epidemiological surveys in the general population. Hence, the prevalence of NPP in the population has mainly been estimated on the basis of studies $[3,11]$ conducted by specialized centers that focus on specific NPP-conditions; such as failed back surgery syndrome (FBSS) [12-15], complex regional pain syndrome (CRPS) [16-19], postherpetic neuralgia [20, 21], painful diabetic polyneuropathy [22-24], post-surgery neuropathic pain [25], spinal cord injury [26], stroke [27] and cancer [28, 29].

Chronic NPP tends to be more frequent in women ( $8 \%$ versus $5.7 \%$ in men) and in patients over 50 years of age ( $8.9 \%$ versus $5.6 \%$ in those $<49$ years of age). It most commonly affects the lower back and lower limbs, and neck [30]. Lumbar and cervical painful radiculopathies are estimated to be the most frequent cause of chronic NPP. In line with this, a survey of $>12,000$ patients with chronic pain with both nociceptive and neuropathic pain types, referred to pain specialists in Germany, revealed that approximately $40 \%$ of all patients experienced at least some characteristics of NPP (such as burning sensations, stabbing sensations, numbness and tingling). Patients with chronic back pain and radiculopathy were particularly affected by the aforementioned symptoms [31]. The incidence rate of NPP is 8.2 per 1000 [32], and is expected to significantly increase in the near future as the Western 
population is aging. Treatment remains challenging as it is often refractory to first-line pharmacological treatment [3, 4, 8, 32-34] and NPP-management should always be placed in context of its underlying cause and aberrant physiological mechanisms. As pharmaceutical treatment of neuropathic pain disorders such as CRPS and FBSS is often limited and accompanied by severe side effects, interventional neuromodulation techniques, such as Tonic SCS, have become a well-established last resort treatment option for the aforementioned intractable neuropathic pain disorders [35-48].

\subsubsection{Failed Back Surgery Syndrome (FBSS)}

FBSS is a term used to describe a clinical entity subsequent to the advent of spinal surgery [49]. The term FBSS is used to describe a constellation of conditions characterized by persistent or recurring low back pain, with or without sciatica following spine surgery. [50]. Follett described FBSS in 1993 as the "surgical end stage after one or several interventions on the lumbar neuraxis indicated to relieve lower back pain, radicular pain or the combination of both, without effect" ( Follett, 1983). [51]. FBSS has a prevalence of approximately $0.61 \%$ of the general population, which makes up approximately 45 million people worldwide [12]. With the number of spine surgeries steadily increasing, the prevalence of FBSS is only expected to rise $[52,53]$. Symptoms usually include a sharp, regular or recurring pain shooting or radiating down one or both legs, potentially accompanied by, partial, muscular dysfunction. The pharmacological treatment of neuropathic pain symptoms in FBSS patients follows a standardized treatment algorithm, and may include a wide variety of commercially available pain killers (such as paracetamol), antidepressants, anticonvulsants, N-methyl-D-aspartate antagonists, and opioids [34, 54]. Although these drugs have proven to alleviate up to $50 \%$ of neuropathic pain symptoms in FBSS patients, pharmacological treatment is often accompanied by (severe) side effects, such as sedation, cardiac arrhythmias, postural hypotension, and behavioral changes [5557]. Furthermore, a subset of FBSS patients do not receive adequate pain relief (less than $50 \%$ ) with pharmacological treatment. Therefore, more invasive and expensive treatment options like stimulation of the dorsal columns of the spinal cord (SCS) are frequently applied $[38,50,58,59]$. 


\subsubsection{Complex regional pain syndrome (CRPS)}

CRPS type 2, or causalgia, is a condition that generally results from (surgical) injury to somatosensory nerves [60]. The most common triggers are fractures, sprains/strains, soft tissue injury, limb immobilization (due to a cast), surgery, or even minor medical procedures such as needle prick. Yet, it is not uncommon that the precipitating event cannot clearly be identified. CRPS is an aberrant response that magnifies the normal physiological response to an injury and is characterized by a rapid onset (usually 24 hours) of spontaneous constant burning pain that is exacerbated by several factors such as light touch (allodynia), changes in temperature and/or movement of the affected limb [61, 62]. Apart from the aforementioned neuropathic symptoms, studies have also proposed neurogenic inflammation as an underlying mechanism for a subset of CRPS-symptoms [63, 64]. CRPS syndrome can be divided into two types:

CRPS Type 1; absence of any form of nerve injury

CRPS Type 2; lesion to a major nerve has been identified

The reported incidence of CRPS is highly variable. Among the Dutch population the estimated incidence of CRPS was reported to be 26.2 per 100,000 [16]. Like most syndromes that are neuropathic in nature, CRPS type I responds poorly to conventional pharmacological analgesic therapy. Besides conventional pain medication, physical therapy, sympathetic blocks, and transcutaneous electrical stimulation of nerves have all been applied in an attempt to reduce the intensity of pain, generally with suboptimal results. Only one in five affected patients is able to return to a normal level of functioning [65]. As a consequence, adequate treatment of CRPS often requires more invasive treatment options like SCS [43]. 


\subsection{Tonic Spinal Cord Stimulation in neuropathic pain}

Spinal cord stimulation requires surgical placement of electrodes on top of the dorsal columns of the spinal cord. Electrodes are carefully inserted, either transcutaneous or via laminectomy, in the epidural space on top of the dura mater surrounding the spinal cord. Then, electrical pulses are administered to the dorsal columns of the spinal cord, via an implantable pulse generator or an external stimulation device. Tonic, conventional, SCS (Tonic SCS) settings vary within a range of $30-80 \mathrm{~Hz}, 100-500 \mu$ s pulse width, and amplitude above sensory threshold $[66,67]$. The concept of Tonic SCS emerged as a direct spin-off from the Gate Control Theory, formulated by Melzack and Wall in 1965 [68, 69]. From this, it was postulated by Shealy, Mortimer and Reswick, that antidromic stimulation of the nonnociceptive $A \beta$-fibers in the dorsal columns could close the 'spinal gate', located in the dorsal horn of the spinal cord. Meanwhile, orthodromic stimulation of the A $\beta$-fibers in the dorsal columns also caused paresthesia (i.e., abnormal tingling sensation) in the area innervated by the stimulated fibers (figure 1). Nowadays, during surgical implantation of the SCS-lead the physician makes sure the paresthesia overlaps the painful area and provides adequate pain relief [70]. Closing of the 'spinal gate' is facilitated by inhibitory interneurons located in the dorsal horn. In line with the Gate Control Theory those inhibitory interneurons, when antidromically activated by Tonic SCS, modulate the nociceptive signal through the release of gamma-amino-butyric acid (GABA). Indeed, experimental research has demonstrated that Tonic SCS decreased intracellular GABAimmunoreactivity in the dorsal horn of chronic neuropathic rats [71]. At the same time, spinal microdialysis in Tonic SCS stimulated chronic neuropathic rats has demonstrated that SCS increases extracellular GABA levels in the dorsal horn of chronic neuropathic rats [7274]. Thus, enhanced GABA release in the dorsal horn seems to be a vital aspect of the mechanisms underlying Tonic SCS. The mechanism of segmental GABA release was further elucidated by the administration of pharmacological agents in chronic neuropathic rats that received Tonic SCS. Local perfusion with a $\mathrm{GABA}_{\mathrm{B}}$-receptor antagonist in the dorsal horn transiently abolished the stimulation-induced effect in neuropathic rats [74], and rats not receiving adequate pain relief with Tonic SCS (non-responders), were turned into responders by administration of the $\mathrm{GABA}_{B}$-receptor agonist baclofen [75]. The aforementioned preclinical findings were successfully translated to the clinic where neuropathic pain patients not responding to Tonic SCS were turned into responders with additional intrathecal administration of subeffective doses of baclofen [76]. 
Experimentally, it has been demonstrated by Smits and colleagues that Tonic SCS results in a much better pain relieving effect when administered at the level where the injured fibers enter the spinal dorsal horn, as compared to SCS administered at more rostral levels [77]. This emphasized the fact that Tonic SCS in treatment of neuropathic pain primarily acts through a segmental, spinal, site of action (see Figure 1).Yet, besides the segmental mode of action, Tonic SCS also invokes activation of supraspinal mechanisms, via orthodromic activation of the $A \beta$-fibers (via the so-called supraspinal feedback loop). Once activated, these supraspinal cell regions modulate the incoming nociceptive signals at the spinal level via descending fiber projections. Brainstem nuclei such as the locus coeruleus and the raphe nucleus, are activated by Tonic SCS and in their turn modulate the spinal pain signal via descending adrenergic and serotonergic projections respectively [78, 79]. Moreover, it has been shown that SCS can modulate activation patterns in brain areas at a subcortical and cortical level. Early clinical fMRI-work on the supraspinal effects of Tonic SCS has demonstrated modulation of brain regions associated with the lateral spinothalamic tract (STT) [80-82]. The lateral STT is responsible for the transmission of objective pain-aspects such as the intensity and location of the painful stimulus. This lateral STT pathway projects from the dorsal horn, via the thalamus, to cortical areas such as the somatosensory cortex $[83,84]$. An fMRI study performed in eight patients receiving Tonic SCS demonstrated that Tonic stimulation increased Blood Oxygen Level-Dependent (BOLD)-signals in somatosensory cortices, the sensorimotor cortex and the insula [81]. Furthermore, a more recent fMRI-study with twenty patients who received Tonic-SCS as treatment for FBSS, reported deactivation of the bilateral medial thalamus and its connections to the rostral and caudal cingulate cortex, and the insula [80]. In conclusion, over the years, literature on Tonic SCS has provided evidence for spinal as well as supraspinal mechanisms. Yet, the principal mechanism of action seems to be situated at a segmental, spinal, level [77]. 


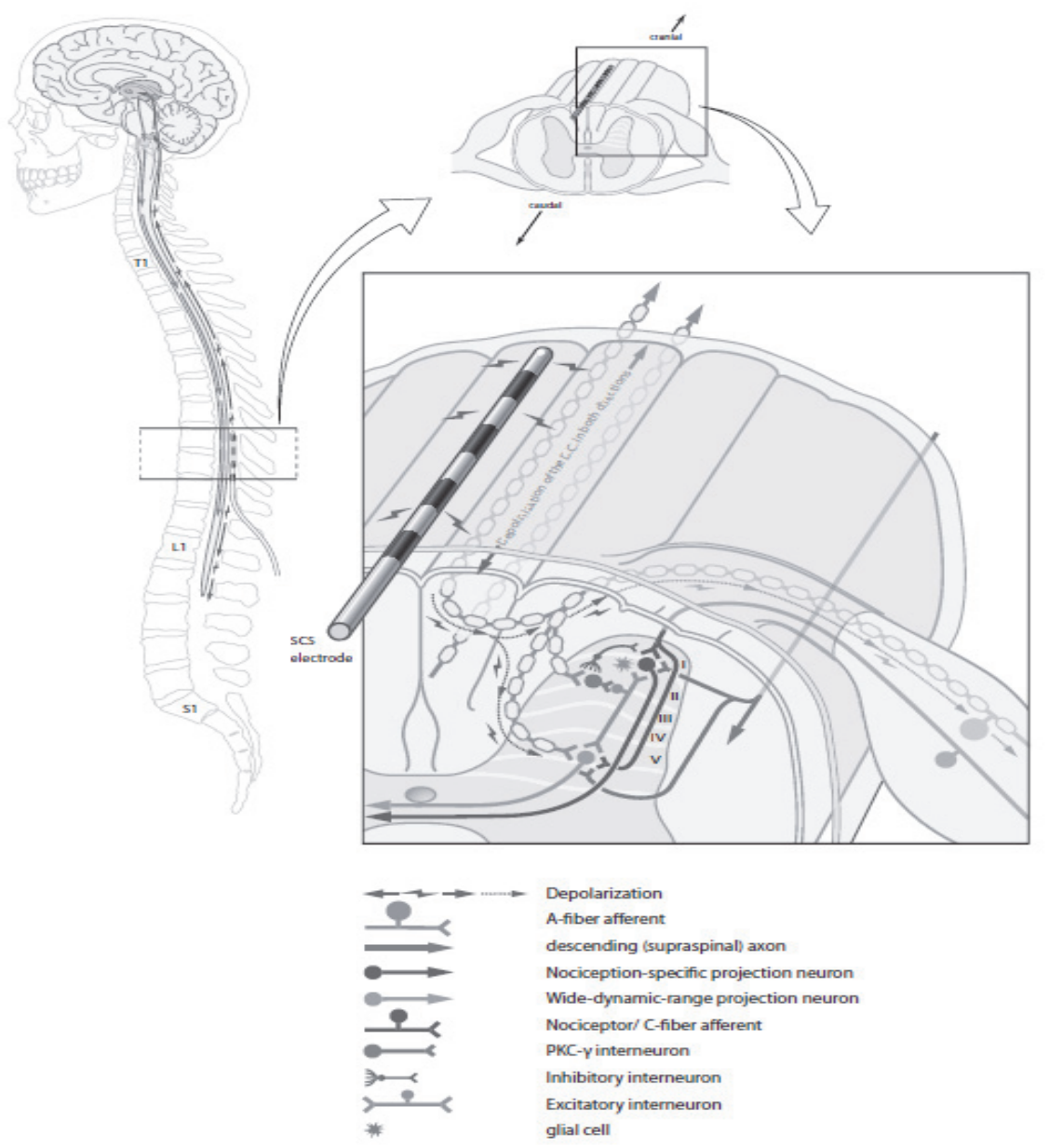

Figure 1: A representation of the spinal nociceptive network and mechanism of Spinal Cord Stimulation (SCS). The dorsal horn of the spinal cord contains two major types of projection neurons: the Nociceptive-Specific projection neurons located in the superficial laminae I, and the Wide Dynamic Range projection neurons located in the deeper laminae (IV, V and VI). These projection neurons receive input from primary afferents, descending (aminergic) pathways, and spinal interneurons. The spinal nociceptive network contains numerous interneurons, both of excitatory and inhibitory (gamma-amino-butyric acid [GABA]-ergic) nature, which modulate the processing of pain signals at the "gate" to the brain ("Gate-Control theory"). Electrical stimulation of the DCs results in an action potential propagating in both directions: orthodromically in rostral direction to supraspinal areas, and antidromically via $A \beta$ fibers back into the spinal cord nociceptive network. Antidromic stimulation of the $A \beta$ fibers results in changed (decreased) release of glutamate and at the same time an increased release of the inhibitory neurotransmitter GABA. From Smits et al. (2012) with permission [85]. 


\subsection{Novel Spinal cord stimulation paradigms}

Despite the many successes that have been achieved with Tonic-SCS, including treatment of various chronic neuropathic pain conditions such as FBSS and CRPS, up to $30 \%$ of patients remain unresponsive to SCS treatment, or experience loss of adequate pain relief over time [35-37, 40, 42, 44-47, 86-93]. In 2012, prospective long-term follow-up data on SCS in CRPSI revealed that $63 \%$ of patients still received adequate pain relief with SCS-treatment 12 years after implantation of the device [45]. However, $59 \%$ of patients experienced less than $30 \%$ of pain relief at the last follow-up measurement as compared to pain scores reported at first intake. For FBSS, two Randomized Clinical Trials (RCT's) have reported that $58 \%$ and $47 \%$ of patients who received SCS-treatment for FBSS experienced adequate pain relief at 24 and 36 months follow-up respectively $[58,59]$. In general, the reported success rate for Tonic SCS has remained suboptimal, with roughly $50 \%$ pain relief on the VAS/NRS score for approximately $50-70 \%$ of the patients $[90,94]$. As a direct consequence, the interventional SCS-field started to investigate novel targets and novel SCS-paradigms that might further improve or sustain pain relief.

A recently introduced novel target for SCS treatment is the dorsal root ganglion (DRG). In contrast to dorsal column SCS, the electrode is steered toward the lateral epidural space near the DRG. It has been found that DRG stimulation offers several potential benefits over Tonic dorsal column SCS, including a more targeted approach with paresthesia and the ability to stimulate areas that are difficult to reach with dorsal column SCS, such as the low back and foot [95-99].

Next to novel physiological targets for SCS, novel SCS-paradigms were introduced in order to bridge to gap between current and desired pain relief. Two prominent examples, High Frequency SCS (HF SCS) and Burst SCS, were recently introduced in order to optimize the efficacy of SCS-treatment for chronic neuropathic pain [67, 89, 100-105]. Both Burst SCS and HF SCS are generally applied at stimulation amplitudes below sensory threshold, which means the patient does not experience paresthesia during stimulation. This has offered researchers, the opportunity to perform double-blind placebo-controlled clinical studies, for the first time since SCS was introduced in 1967 [41, 106-108]. HF SCS is generally applied at a frequency above $1000 \mathrm{~Hz}$, up until $10 \mathrm{kHz}$, with a pulse width at approximately 30 microseconds ( $\mu \mathrm{s})$ and amplitude typically 1-5 mA [109]. Hypotheses about the underlying mechanism of HF SCS vary. It is suggested that 1 ) temporal summation could play a role, where multiple pulses build on each other to achieve neuronal activation; 2 ) depolarization blockade could occur, where propagating action potentials are blocked by the high 
frequency stimulation; and 3) desynchronization could result in pseudo-spontaneous or stochastic neuronal activity in the spinal "gate" [110-112]. Till today, the optimal frequency for HF SCS has not yet been determined, and clinical evidence suggests that different HFfrequencies, ranging from $1000 \mathrm{~Hz}$ to $10 \mathrm{Khz}$, can yield clinically significant pain relief [100, $102,108,109,113-115]$.

The Burst-paradigm was introduced in 2010 by de Ridder and colleagues as 'BurstDR'SCS $[103,104,116]$. The BurstDR-waveform consists of five closely spaced monophasic spikes administered at $40 \mathrm{~Hz}$ inter-burst mode and $500 \mathrm{~Hz}$ intraburst frequency, with a pulse width of $1 \mathrm{~ms}$ and $1 \mathrm{~ms}$ interspike interval, delivered in constant current mode. The cumulative charge of the five $1 \mathrm{~ms}$ spikes is balanced during the $5 \mathrm{~ms}$ after the spikes, in a so called passive recharge phase, which differentiates it from HF SCS and Tonic SCS, in which each pulse is immediately charge balanced after each spike, in a so called active recharge phase $[104,116]$. The BurstDR-pattern was chosen because it supposedly mimics naturally occurring neural bursting patterns in the central nervous system. Indeed, neurons responsible for encoding aspects of pain signaling from peripheral neurons [117-119] and the thalamus [120-122] have been reported to fire in bursting patterns. However, it is important to mention that "BurstDR ${ }^{\mathrm{TM}}$ " is a registered trademark and only one of an infinite number of possible Burst waveforms [123]. Interestingly, like HF SCS, the Burst paradigm has been reported to produce pain relief without inducing paresthesia in the majority of patients. Paresthesia is the sensation that results from activation of sensory-specific dorsal column fibers [124], so absence of paresthesia suggests that stimulation is not activating dorsal column $A \beta$-fibers $[125,126]$. However, even though stimulation at low amplitude may be subthreshold with respect to neuronal activation, and sub-perception with respect to the patient's experience, large amounts of charge are still delivered to dorsal horn fibers, providing the pulse width and/or frequency are sufficiently large [112]. This could potentially set in motion, additional, dorsal horn mechanisms that are not activated with suprathreshold Tonic SCS. Yet, the key difference between Tonic-SCS and Burst SCS is thought to be located higher up the neuraxis, at a supraspinal level. Clinical evidence suggests that Burst SCS, unlike Tonic SCS, activates brain areas associated with the processing of cognitive-emotional aspects of pain such as the amygdala, the anterior cingulate cortex, and the insula. The aforementioned brain areas are linked to the medial STT [116, 127] (Figure 2). In 2013, a small clinical study demonstrated that patients who received BurstDR SCS improved on pain aspects like 'the amount of attention the patients pay to pain' and 'changes in pain' in a statistically significant way, as measured by the Pain Vigilance and Awareness Questionnaire (PVAQ) [116]. Meanwhile, patients who received 
Tonic or placebo stimulation showed no significant effect on the aforementioned pain aspects. Subsequently, a source-localized electroencephalography study in patients who received Burst or Tonic SCS showed that Burst SCS was able to normalize the pain supporting/ suppressing balance by having a greater effect on the dorsal anterior cingulate cortex [127]. This further supports the notion that Burst SCS has an effect on brain areas associated with the processing of cognitive-emotional aspects of pain.

Although the aforementioned studies have helped shed light on the (supraspinal) mechanisms underlying Burst SCS, the preponderance of mechanisms underlying the pain relieving effect of Burst SCS is still largely unknown. It is a firm belief that it is pivotal that more insight is acquired into the role of supraspinal brain areas associated with the pain relieving effect of (Burst and Tonic) SCS.

\subsubsection{Burst SCS: clinical evidence}

Early clinical studies have demonstrated that BurstDR stimulation is able to provide clinically relevant pain relief, which, in a subset of patients, may surpass the pain relief provided by Tonic stimulation $[41,116,128,129]$. Although additional pain reduction during BurstDR stimulation was found to be only marginally greater as compared with Tonic stimulation, BurstDR stimulation was preferred by the majority of patients. The preference for BurstDR stimulation is most likely correlated to the lack of paresthesia. It has been demonstrated that BurstDR SCS produces paresthesia only in a subset of patients, approximately $17-25 \%$, and anecdotal reports indicate that paresthesia during BurstDR stimulation may be perceived as qualitatively different from paresthesia experienced during Tonic SCS [104, $128,129]$. Additionally, there are studies that suggest the ability of BurstDR SCS to recover patients that no longer achieve adequate pain relief with Tonic SCS anymore, as well as the ability to reduce the affective aspects of chronic neuropathic pain $[104,116,130]$. Yet, these early studies evaluating Burst SCS enrolled relatively small cohorts of patients, over a short period of time, and there remained a lack of randomized, placebo-controlled clinical trials. Therefore, a larger controlled study was necessary in order to demonstrate the safety and efficacy of BurstDR stimulation, compared to Tonic stimulation. The primary objective of the 'success using neuromodulation with Burst' (SUNBURST) study was to establish the noninferiority of pain intensity after three months of Burst SCS compared to three months of Tonic SCS [131]. The SUNBURST study demonstrated that BurstDR SCS is indeed noninferior to Tonic SCS, and superiority of BurstDR SCS was also achieved. In addition, significantly more subjects (70.8\%) preferred BurstDR stimulation over Tonic stimulation, and this preference was sustained throughout the first year: $68.2 \%$ of subjects preferred 
BurstDR stimulation, $23.9 \%$ of subjects preferred Tonic stimulation, and $8.0 \%$ of subjects had no preference. At the same time, evidence suggests that pain relief with BurstDR SCS is not necessarily better than with Tonic-SCS, and different SCS-modalities might be preferred with different indications $[131,132]$. Such contradictory findings suggest that Burst SCS still remains to be optimized and to this end more research into the underlying mechanisms is required. Parameter variations within the Burst waveform, that would require specific deviations from the patented BurstDR-waveform, could potentially generate additional pain relief. A few studies have attempted to elucidate the mechanisms, and/or optimize the parameters, of Burst SCS. With respect to the mechanisms underlying BurstDR SCS potential immunomodulatory properties were investigated in a small cohort of CRPS-patients [133]. It was found that BurstDR SCS, but also Tonic SCS and HF SCS attenuates T-cell activation, improves peripheral tissue oxygenation and decreases anti-angiogenetic activity. The possible immunomodulatory effects of SCS could bring new therapeutic possibilities in diseases that involve the immune system, and deserves further research [133]. Havenbergh and colleagues investigated the effect of parameter changes on the analgesic efficacy of BurstDR SCS [134]. Fifteen patients who were being treated with Burst SCS for failed back surgery syndrome participated to verify whether their pain suppression could be further improved by changing the Burst pattern. Burst stimulation with packets of five electrical pulses delivered at $500 \mathrm{~Hz}$ with $1000-\mu \mathrm{sec}$ pulse width 40 times per second (BurstDR) was compared to an alternate Burst mode delivering five spikes at $1000 \mathrm{~Hz}$ with 500 - $\mu \mathrm{sec}$ pulse width 40 times a second. No statistically significant differences in pain relief were found between the two modes of Burst stimulation. The authors therefore concluded that increasing the frequency from 500 to $1000 \mathrm{~Hz}$ does not add any extra benefit in suppressing pain. Further studies should verify whether adjusting other parameters within the Burst design might have an (beneficial) effect on its efficacy. Currently, studies are being performed in which patients receive an alternate Burst mode which differs from BurstDRSCS in that charge-balancing is performed directly, during the intraburst period, in a socalled active recharge manner. At this point in time, no clear consensus has been reached regarding the optimal Burst-paradigm, and no data from clinical or preclinical studies support the claim that either passive recharge Burst SCS (BurstDR) or active recharge Burst SCS is superior $[123,135]$. Therefore, further research into, and exploration of, new and innovative Burst paradigms is required and might help pave the way towards better pain relief. 


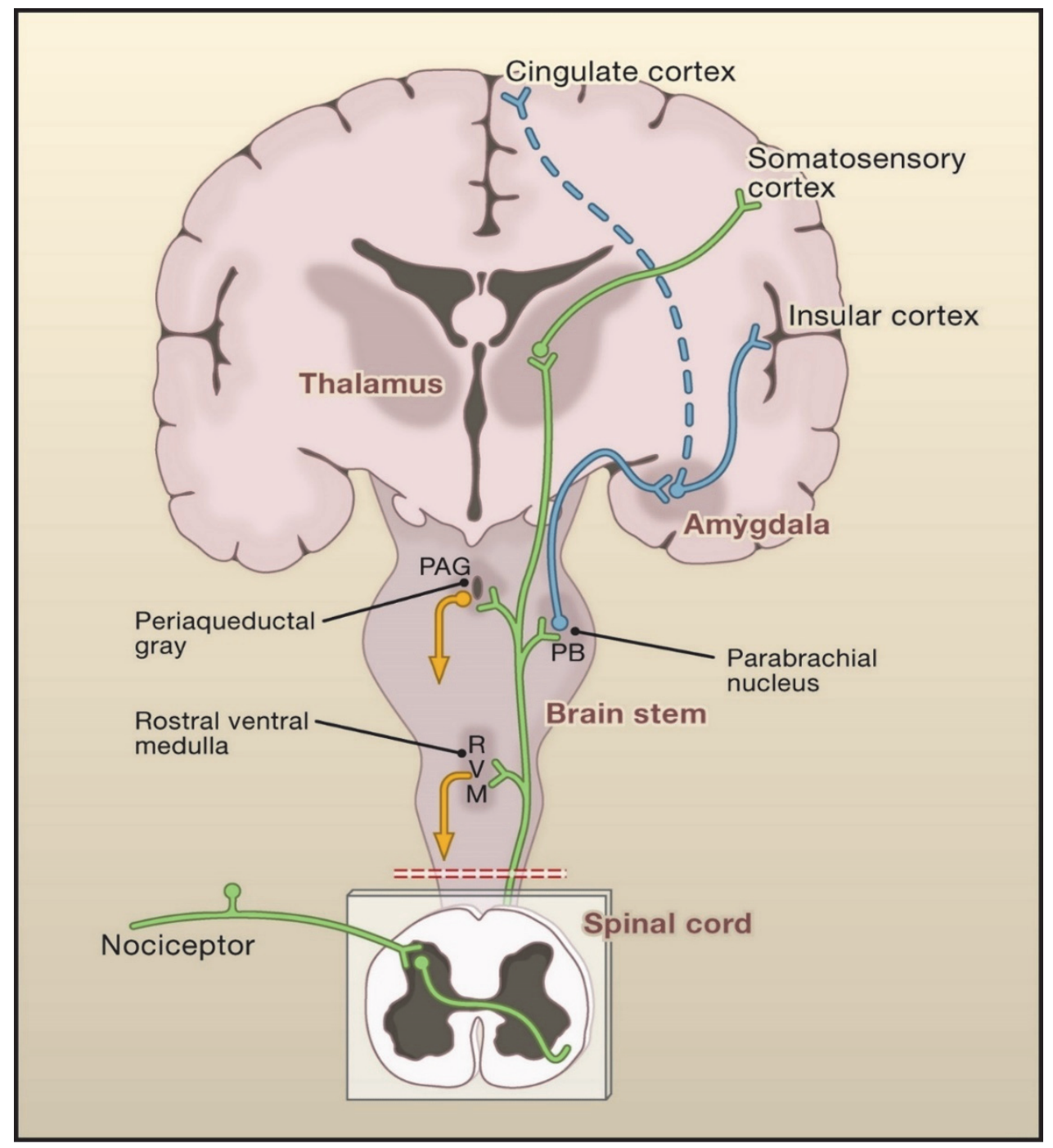

Figure 2. Anatomy of the Pain Pathways: Primary afferent nociceptors transmit noxious information to projection neurons in the dorsal horn of the spinal cord. A subset of these projection neurons transmits information via the lateral spinothalamic tract (depicted in green) via the thalamus to the somatosensory cortex, providing information about the location and intensity of the painful stimulus. Other projection neurons send nociceptive signals along the medial spinothalamic tract (depicted in blue), which runs via connections in the brainstem (parabrachial nucleus) to the cingulate and insular cortices, and the amygdala, contributing to the affective component of the pain experience. Meanwhile, ascending information also activates neurons of the rostral ventral medulla and midbrain periaqueductal gray, which results in descending feedback able to regulate nociceptive processing in the spinal cord. From Basbaum et al. (2009) with permission ([84]. 


\subsubsection{Burst Spinal Cord Stimulation: experimental}

A few experimental studies have aimed to investigate the mechanisms underlying the analgesic effects of Burst SCS in neuropathic pain. In a preclinical setting, it was shown that the presence of a GABA ${ }_{B}$-receptor antagonist CGP35348 blocked attenuation of dorsal horn neuronal firing during Tonic SCS (see section 1.3) but not Burst SCS. From this, the authors concluded that Burst SCS might not act via spinal GABAergic mechanisms [136]. However, it should be mentioned that the aforementioned study was performed not only in a most uncommon rat model for chronic neuropathic pain, the Painful Cervical Nerve Root Compression model, but these experiments were also terminal and thus no behavioral testing during the conscious state of the animals was performed [31]. This makes interpretation of these data in the context of understanding the role of spinal GABA in a chronic neuropathic pain model difficult, as most experimental data on pain relief and the spinal GABAergic mechanism underlying Tonic SCS have been documented and studied in the well-described and validated partial sciatic nerve ligation (PSNL) model or a similar nerve injury model for peripheral mononeuropathy. Therefore, the question remains if and how Burst SCS acts on GABAergic mechanisms at the spinal level when using a well-validated and widely used chronic neuropathic pain model that allows for comparison with past literature (demonstrating the role of spinal GABA release in the mechanism of Tonic SCS) $[71,74,137]$. In 1990, Seltzer and colleagues presented a behavioral model of causalgia in rats: the partial ligation of the sciatic nerve (PSNL). The PSNL-model was originally introduced as a behavioral model of CRPS (causalgia) (see section 1.2.2), though it was soon after acknowledged as an animal model for FBSS (see section 1.2.1). The model shows many of the symptoms that characterize neuropathic pain in humans, including rapid onset of allodynia to touch, hyperalgesia, spontaneous occurrence of pain, and mirror pain. The plantar surface of the animals' paw is hyperesthetic to non-noxious and noxious stimuli, and the hyperestheticy is evenly distributed. These symptoms are characterized by a sharp decrease in the withdrawal thresholds in response to repetitive Von Frey hair stimulation at the plantar side. Light touch with innocuous stimuli elicit aversive responses, which suggests allodynia to touch, and suprathreshold noxious stimuli elicit an exaggerated response unilaterally, which suggests hyperalgesia [62]. Furthermore, Seltzer and colleagues saw that none of the animals showed signs of autotomy, which was a major improvement as compared to existing animal models for chronic NPP at that time [2,138, 139]. Based on this, it was concluded that the PSNL-model was a valid model for syndromes of the causalgiform variety that are triggered by partial nerve injury and maintained by sympathetic activity. Since its introduction in 1990, the PSNL-model has become a 
renowned and widely recognized animal model for CRPS and FBSS which is reflected by its use inside, and outside, the SCS-field [2, 71, 74, 77, 138, 140-143].

Experimental electrophysiological studies indicate that Burst SCS can be optimized by adjusting stimulation parameters closely related to the energy delivered to the spinal cord, such as amplitude and pulse width, with reports that the energy delivered to the spinal cord was found to be correlated with a reduction of 'wide dynamic range neuronal' firing in the dorsal horn following noxious stimuli [126]. Other studies have shown that Burst-SCS and Tonic SCS have a different effect on the suppression of lumbosacral neural responses when applied with a high-intensity ( $90 \% \mathrm{MT})$ when compared with standard-intensity (60\% MT) [125]. However, the aforementioned studies did not assess the behavioral effect of parameter variations on the pain relieving efficacy of Burst SCS. In general, on a behavioral level, the literature remains rather restricted, with only one study demonstrating that BurstSCS reduces hyperalgesia and restores physical activity levels more so than Tonic SCS in the spared nerve injury model[144]. Hence, a better understanding of the behavioral antinociceptive effect of Burst SCS as compared to Tonic SCS in an experimental neuropathic pain model is needed. This is important in view of further optimization of the Burst-SCS paradigm and then also might have serious implications for its clinical use in the treatment of neuropathic pain. Furthermore, literature shows that behavioral analysis and thus assessment of the pain relieving effect is known to be the most important indicator of a treatment's translational value [138]. Therefore, it is imperative that parameter variations are always assessed by means of behavioral testing before translation towards the clinical setting is considered.

Testing of reflexes such as paw withdrawal testing is well established as the standard for evaluating nociceptive behavior and its modulation in preclinical animal studies [145]. Concerns about the extensive use of, and overreliance on, this approach have been dismissed for practical reasons: reflex-based testing requires no training of the animals, and the method is simple to instrument. Yet, many are frustrated with the lack of translational progress in the pain field, in which huge gains in basic science knowledge obtained using animal models have not led to the development of proportionate new clinically effective compounds [138]. It has been suggested that the use of operant testing methods, such as place preference and escape testing, in addition to reflex-based assessment methods could aid translation to the clinic $[145,146]$ : comparison of studies that applied either reflexbased testing or operant escape measures as assessment method for pain showed that 
results of operant testing measures were more often consistent with clinical evidence [145]. Though, to this day, the majority of preclinical SCS studies still rely on reflex-based von Frey analysis, a technique unable to assess supraspinal aspects of pain [77, 141-144, 147-150].

Meanwhile, the preclinical pain-field has ventured into the domain of operant testing. Learned escape from electric footshock in shuttle boxes for example is a very old technique [151], often used in studies of learning and memory, yet, also used to study pain itself. Conflicts between positive and negative reinforcement have long been exploited for operant testing methods, with modern versions featuring painful stimuli or positioning of inflamed or neuropathic body parts as the price for food or water [152-154]. Self-administration of analgesic drugs has been used as an indirect measure of pain [155], and conditioned place preference (to an analgesic in the presence of pain159) or conditioned place aversion strategies have been applied to investigate pain behavior [156158]. Recently, an operant testing method was introduced which assesses cognitive and motivational aspects of pain in animals: the Mechanical Conflict-Avoidance System (MCAS) [159]. With the MCAS the animal is placed in a brightly lit compartment which leads to a passage with a height-adjustable array of nociceptive probes. The animal needs to cross the nociceptive probes to enter the innately preferred dark area. The "lesser of two evils principle" forces the animal to choose between two opposing motivational drives: to stay in the aversive, yet non-noxious, brightly lit compartment, or, to cross the noxious probes, which is rewarded by the innately preferred dark compartment. In order to resolve this conflict it is hypothesized that the animal applies a "cost-benefit" analysis including the level of ongoing pain, the height of the probes, and the averseness of the light (= negative reinforcement) [160]. In general, as ongoing pain intensity and/ or probe height increases, animals require more time to exit the light chamber. Latency to exit the light chamber (defined as time from light being turned on to having all four paws on the probe bed), as a behavioral representation of cognitive-motivational aspects of pain, has been shown to be a stimulus-dependent measure in the Coy-MCAS system. Chronic neuropathic pain, induced by chronic constriction of the sciatic nerve, has been shown to affect latency to exit the bright compartment in the MCAS [161]. However, other neuropathic pain models still remain to be validated in the MCAS-setup. The MCAS operant testing system could be an important step towards improved translation in the experimental pain field. Furthermore, with the introduction of SCS-paradigms able to modulate supraspinal properties, it is of great interest to the preclinical SCS-field to assess whether the MCAS can shed light on the supraspinal mechanisms of Burst and Tonic SCS by addressing the cognitive-motivational 
aspects of pain that are becoming increasingly important for the evaluation of novel SCS waveforms.

In a recent review it was hypothesized that HF SCS $(10 \mathrm{kHz})$ and Burst SCS might be fundamentally similar neurostimulation designs [162]. It was stated that clinical data, quantitative sensory testing, cellular investigations, and comparative animal and human studies all point in the same direction, namely that HF SCS and Burst SCS might both modulate the medial STT, and thus, have fundamentally similar working mechanisms. In line with this, a computer modeling study indicated that SCS frequency (at least partly) determines the firing of wide dynamic range (WDR) neurons projecting to the brain [163]. Analysis showed that SCS with frequencies above $100 \mathrm{~Hz}$ does not result in inhibition of WDR signaling; this strongly suggests that higher SCS frequencies, as applied with HF SCS and Burst SCS, induce pain relief via alternative mechanisms. In another study, it was reported that the orthodromic activation of $A \beta$-fibers in the dorsal columns might differ between Burst and Tonic SCS: Burst SCS was found not to increase spontaneous activity of gracile nucleus neurons in the brainstem, whereas Tonic SCS did [125], further supporting the notion that different mechanisms are recruited with different SCS-paradigms. Moreover, a behavioral study reported a delayed wash-in of the analgesic effect of HF SCS (500 Hz), as compared with standard Tonic SCS $(50 \mathrm{~Hz})$, in animals with chronic painful diabetic polyneuropathy [150]. Building on the hypothesis that HF SCS and Burst SCS might be fundamentally similar neurostimulation designs, the delayed onset effect could reflect the activation of the 'slower' medial STT pathway. Investigating the onset effect of Burst SCS by assessment of its analgesic effect over time could shed more light on the aforementioned hypothesis. Interestingly, a potential difference in effect onset for Burst SCS and Tonic SCS would be in line with unpublished clinical anecdotes. 


\subsection{Aims and Research questions}

The primary aim of this academic thesis is to improve the analgesic efficacy of Burst SCS in an experimental neuropathic pain model, based on an improved understanding of the underlying mechanism.

\section{RESEARCH QUESTIONS (RQ)}

Based on the aim of this academic thesis, the following research questions are formulated:

1. What is the effect of amplitude on the analgesic efficacy of Burst- and Tonic SCS in an experimental animal model of chronic neuropathic pain?

As clinical observations suggested a delayed wash-in effect of Burst SCS, and in order to further optimize the Burst SCS paradigm and understand the underlying mechanism, it is important to understand the pain relieving effect in detail, both in time and in magnitude as compared to Tonic SCS. Therefore, we formulated the following RQ:

\section{Does the anti-nociceptive effect over time differ for Burst SCS and Tonic SCS?}

From a mechanistic point of view, and in light of the Gate Control Theory, it is important to elucidate the role of GABA in the modulation of the pain with Burst SCS in a wellvalidated and widely used animal model of chronic neuropathic pain. Hence, we formulated the next RQ:

\section{Is GABA involved in the spinal pain relieving mechanism of Burst SCS?}

With the introduction of novel SCS-paradigms such a Burst SCS which are suggested to modulate supraspinal (brain) regions, it is of great interest to the preclinical SCS-field to analyze the role of supraspinal regions, and brain areas involved in the pain relieving effect of SCS. It is therefore that we formulated the following research questions:

4. Is it possible to discriminate between Burst and Tonic SCS on cognitive-motivational aspects of pain relief, with use of MCAS operant testing?

5. Which brain areas are involved in the pain relieving effect of Burst SCS, as compared to Tonic SCS, in an animal model of chronic neuropathic pain? 
In order to acquire more insight into the relation between amplitude, a pivotal SCS parameter, and the therapeutic analgesic effect of Burst SCS, we studied behavioral effects of chronic neuropathic animals in response to various amplitudes of Burst SCS and Tonic SCS. This study will be addressed in Chapter 2 (see RQ 1 ).

With RQ2 we investigated the effect of Burst SCS and Tonic SCS on mechanical hypersensitivity during stimulation (wash-in and maximum effect; 60 minutes), and 30 minutes post stimulation (wash-out effect). The mechanisms responsible for the difference in delayed wash-in of the pain relieving effect observed for Burst SCS as compared to Tonic SCS is described in Chapter 3.

Insight into the spinal GABAergic mechanisms of Burst SCS will be provided in Chapter 4 (see RQ3). By use of quantitative immunohistochemical analysis of intracellular GABA-levels in the dorsal spinal cord of chronic neuropathic rats, and intrathecal administration of pharmacological agents (phaclofen and baclofen) prior to stimulation, we were able to investigate the spinal GABAergic mechanisms responsible for the analgesic effect of Burst SCS and Tonic SCS. These experiments were performed in a well-established chronic neuropathic animal model, and are further described in Chapter 4.

The behavioral effect of Burst SCS and Tonic SCS on cognitive-motivational aspects of pain were investigated in a recently developed operant testing-system known as the Mechanical Conflict Avoidance (MCAS) setup (RQ4). Use of Burst SCS or Tonic SCS in the MCAS-setup allowed us to study its effect on escape latencies; a behavioral measurement of cognitivemotivational pain processing. This study will be addressed in Chapter 5 .

In order to investigate which brain areas are involved in the establishment of the pain relieving effect of Burst SCS, as compared to Tonic SCS (see RQ5), we used fMRI in order to assess the Blood-Oxygenation-Level Dependent (BOLD) levels in the brains of chronic neuropathic animals that received SCS in the scanner. The results are presented in Chapter 6. 


\section{References}

1. Charlton, J.E., Neuropathic Pain Core Curriculum for Professional Education in Pain, 2005. IASP Press, Seattle.

2. Jaggi, A.S., V. Jain, and N. Singh, Animal models of neuropathic pain. Fundam Clin Pharmacol, 2011. 25(1): p. 1-28.

3. Colloca, L., et al., Neuropathic pain. Nat Rev Dis Primers, 2017. 3: p. 17002.

4. Woolf, C.J. and R.J. Mannion, Neuropathic pain: aetiology, symptoms, mechanisms, and management. Lancet, 1999. 353(9168): p. 1959-64.

5. Jensen, T.S., et al., A new definition of neuropathic pain. Pain, 2011. 152(10): p. 2204-5.

6. Costigan, M., J. Scholz, and C.J. Woolf, Neuropathic pain: a maladaptive response of the nervous system to damage. Annu Rev Neurosci, 2009. 32: p. 1-32.

7. Backonja, M.M. and B.S. Galer, Pain assessment and evaluation of patients who have neuropathic pain. Neurol Clin, 1998. 16(4): p. 775-90.

8. Attal, N., et al., The specific disease burden of neuropathic pain: results of a French nationwide survey. Pain, 2011. 152(12): p. 2836-43.

9. Meyer-Rosberg, K., et al., Peripheral neuropathic pain--a multidimensional burden for patients. Eur J Pain, 2001. 5(4): p. 379-89.

10. Torrance, N., et al., The epidemiology of chronic pain of predominantly neuropathic origin. Results from a general population survey. J Pain, 2006. 7(4): p. 281-9.

11. van Hecke, O., et al., Neuropathic pain in the general population: a systematic review of epidemiological studies. Pain, 2014. 155(4): p. 654-62.

12. Thomson, S. and L. Jacques, Demographic characteristics of patients with severe neuropathic pain secondary to failed back surgery syndrome. Pain Pract, 2009. 9(3): p. 206-15.

13. Thomson, S., Failed back surgery syndrome - definition, epidemiology and demographics. Br J Pain, 2013. 7(1): p. 56-9.

14. Inoue, S., et al., Prevalence, characteristics, and burden of failed back surgery syndrome: the influence of various residual symptoms on patient satisfaction and quality of life as assessed by a nationwide Internet survey in Japan. J Pain Res, 2017. 10: p. 811-823.

15. Taylor, R.S. and R.J. Taylor, The economic impact of failed back surgery syndrome. Br J Pain, 2012. 6(4): p. 174-81.

16. de Mos, M., et al., The incidence of complex regional pain syndrome: a population-based study. Pain, 2007. 129(1-2): p. 12-20.

17. Kim, H., et al., Epidemiology of complex regional pain syndrome in Korea: An electronic population health data study. PLoS One, 2018. 13(6): p. e0198147.

18. Elsharydah, A., et al., Complex regional pain syndrome type 1 predictors - Epidemiological perspective from a national database analysis. J Clin Anesth, 2017. 39: p. 34-37.

19. Murphy, K.R., et al., Prevalence of Specific Types of Pain Diagnoses in a Sample of United States Adults. Pain Physician, 2017. 20(2): p. E257-E268.

20. Bouhassira, D., et al., Patient perspective on herpes zoster and its complications: an observational prospective study in patients aged over 50 years in general practice. Pain, 2012. 153(2): p. 342-9.

21. Helgason, S., et al., Prevalence of postherpetic neuralgia after a first episode of herpes zoster: prospective study with long term follow up. BMJ, 2000. 321(7264): p. 794-6.

22. Daousi, C., et al., Chronic painful peripheral neuropathy in an urban community: a controlled comparison of people with and without diabetes. Diabet Med, 2004. 21(9): p. 976-82.

23. Davies, M., et al., The prevalence, severity, and impact of painful diabetic peripheral neuropathy in type 2 diabetes. Diabetes Care, 2006. 29(7): p. 1518-22.

24. Bouhassira, D., M. Letanoux, and A. Hartemann, Chronic pain with neuropathic characteristics in diabetic patients: a French cross-sectional study. PLoS One, 2013. 8(9): p. e74195. 
25. Jensen, M.P., M.J. Chodroff, and R.H. Dworkin, The impact of neuropathic pain on healthrelated quality of life: review and implications. Neurology, 2007. 68(15): p. 1178-82.

26. Siddall, P.J., et al., A longitudinal study of the prevalence and characteristics of pain in the first 5 years following spinal cord injury. Pain, 2003. 103(3): p. 249-57.

27. Klit, H., et al., Central poststroke pain: a population-based study. Pain, 2011. 152(4): p. 818-24.

28. Rayment, C., et al., Neuropathic cancer pain: prevalence, severity, analgesics and impact from the European Palliative Care Research Collaborative-Computerised Symptom Assessment study. Palliat Med, 2013. 27(8): p. 714-21.

29. Bennett, M.I., et al., Prevalence and aetiology of neuropathic pain in cancer patients: a systematic review. Pain, 2012. 153(2): p. 359-65.

30. Bouhassira, D., et al., Prevalence of chronic pain with neuropathic characteristics in the general population. Pain, 2008. 136(3): p. 380-7.

31. Freynhagen, R., et al., Screening of neuropathic pain components in patients with chronic back pain associated with nerve root compression: a prospective observational pilot study (MIPORT). Curr Med Res Opin, 2006. 22(3): p. 529-37.

32. Dieleman, J.P., et al., Incidence rates and treatment of neuropathic pain conditions in the general population. Pain, 2008. 137(3): p. 681-8.

33. Dworkin, R.H., et al., Recommendations for the pharmacological management of neuropathic pain: an overview and literature update. Mayo Clin Proc, 2010. 85(3 Suppl): p. S3-14.

34. Finnerup, N.B., et al., Algorithm for neuropathic pain treatment: an evidence based proposal. Pain, 2005. 118(3): p. 289-305.

35. Hou, S., K. Kemp, and M. Grabois, A Systematic Evaluation of Burst Spinal Cord Stimulation for Chronic Back and Limb Pain. Neuromodulation, 2016. 19(4): p. 398-405.

36. Sdrulla, A.D., Y. Guan, and S.N. Raja, Spinal Cord Stimulation: Clinical Efficacy and Potential Mechanisms. Pain Pract, 2018. 18(8): p. 1048-1067.

37. Reig, E. and D. Abejon, Spinal cord stimulation: a 20-year retrospective analysis in 260 patients. Neuromodulation, 2009. 12(3): p. 232-9.

38. Kumar, K., et al., Spinal cord stimulation versus conventional medical management for neuropathic pain: a multicentre randomised controlled trial in patients with failed back surgery syndrome. Pain, 2007. 132(1-2): p. 179-88.

39. Health Quality, O., Spinal cord stimulation for neuropathic pain: an evidence-based analysis. Ont Health Technol Assess Ser, 2005. 5(4): p. 1-78.

40. Poree, L., et al., Spinal cord stimulation as treatment for complex regional pain syndrome should be considered earlier than last resort therapy. Neuromodulation, 2013. 16(2): p. 12541.

41. Schu, S., et al., A prospective, randomised, double-blind, placebo-controlled study to examine the effectiveness of burst spinal cord stimulation patterns for the treatment of failed back surgery syndrome. Neuromodulation, 2014. 17(5): p. 443-50.

42. Kemler, M.A., et al., Spinal cord stimulation in patients with chronic reflex sympathetic dystrophy. N Engl J Med, 2000. 343(9): p. 618-24.

43. Grabow, T.S., P.K. Tella, and S.N. Raja, Spinal cord stimulation for complex regional pain syndrome: an evidence-based medicine review of the literature. Clin J Pain, 2003. 19(6): p. 37183.

44. Taylor, R.S., J.P. Van Buyten, and E. Buchser, Spinal cord stimulation for complex regional pain syndrome: a systematic review of the clinical and cost-effectiveness literature and assessment of prognostic factors. Eur J Pain, 2006. 10(2): p. 91-101.

45. Geurts, J.W., et al., Spinal cord stimulation for complex regional pain syndrome type l: a prospective cohort study with long-term follow-up. Neuromodulation, 2013. 16(6): p. 523-9; discussion 529. 
46. Kemler, M.A., et al., Effect of spinal cord stimulation for chronic complex regional pain syndrome Type I: five-year final follow-up of patients in a randomized controlled trial. J Neurosurg, 2008. 108(2): p. 292-8.

47. Kemler, M.A., et al., The cost-effectiveness of spinal cord stimulation for complex regional pain syndrome. Value Health, 2010. 13(6): p. 735-42.

48. Urits, I., et al., Complex Regional Pain Syndrome, Current Concepts and Treatment Options. Curr Pain Headache Rep, 2018. 22(2): p. 10.

49. Daniell, J.R. and O.L. Osti, Failed Back Surgery Syndrome: A Review Article. Asian Spine J, 2018. 12(2): p. 372-379.

50. North, R.B., et al., Failed back surgery syndrome: 5-year follow-up in 102 patients undergoing repeated operation. Neurosurgery, 1991. 28(5): p. 685-90; discussion 690-1.

51. Lucas, A.J., Failed back surgery syndrome: whose failure? Time to discard a redundant term. $\mathrm{Br}$ J Pain, 2012. 6(4): p. 162-5.

52. Gray, D.T., et al., Population-based trends in volumes and rates of ambulatory lumbar spine surgery. Spine (Phila Pa 1976), 2006. 31(17): p. 1957-63; discussion 1964.

53. Deyo, R.A., et al., United States trends in lumbar fusion surgery for degenerative conditions. Spine (Phila Pa 1976), 2005. 30(12): p. 1441-5; discussion 1446-7.

54. Jensen, T.S., et al., New perspectives on the management of diabetic peripheral neuropathic pain. Diab Vasc Dis Res, 2006. 3(2): p. 108-19.

55. Rathur, H.M. and A.J. Boulton, Recent advances in the diagnosis and management of diabetic neuropathy. J Bone Joint Surg Br, 2005. 87(12): p. 1605-10.

56. Cohen, K., et al., Pharmacological treatment of diabetic peripheral neuropathy. P T, 2015. 40(6): p. 372-88.

57. Gimbel, J.S., P. Richards, and R.K. Portenoy, Controlled-release oxycodone for pain in diabetic neuropathy: a randomized controlled trial. Neurology, 2003. 60(6): p. 927-34.

58. North, R.B., et al., Spinal cord stimulation versus repeated lumbosacral spine surgery for chronic pain: a randomized, controlled trial. Neurosurgery, 2005. 56(1): p. 98-106; discussion 106-7.

59. Kumar, K., et al., The effects of spinal cord stimulation in neuropathic pain are sustained: a 24month follow-up of the prospective randomized controlled multicenter trial of the effectiveness of spinal cord stimulation. Neurosurgery, 2008. 63(4): p. 762-70; discussion 770.

60. Schwartzman, R.J., K.L. Erwin, and G.M. Alexander, The natural history of complex regional pain syndrome. Clin J Pain, 2009. 25(4): p. 273-80.

61. Thurlow, G. and B. Gray, Complex regional pain syndrome. Int J Orthop Trauma Nurs, 2018. 30: p. 44-47.

62. Seltzer, Z., R. Dubner, and Y. Shir, A novel behavioral model of neuropathic pain disorders produced in rats by partial sciatic nerve injury. Pain, 1990. 43(2): p. 205-18.

63. Birklein, F., et al., The important role of neuropeptides in complex regional pain syndrome. Neurology, 2001. 57(12): p. 2179-84.

64. Bharwani, K.D., et al., Elevated Plasma Levels of sIL-2R in Complex Regional Pain Syndrome: $A$ Pathogenic Role for T-Lymphocytes? Mediators Inflamm, 2017. 2017: p. 2764261.

65. Subbarao, J. and G.K. Stillwell, Reflex sympathetic dystrophy syndrome of the upper extremity: analysis of total outcome of management of 125 cases. Arch Phys Med Rehabil, 1981. 62(11): p. 549-54.

66. Meyerson, B.A. and B. Linderoth, Mode of action of spinal cord stimulation in neuropathic pain. J Pain Symptom Manage, 2006. 31(4 Suppl): p. S6-12.

67. Shechter, R., et al., Conventional and kilohertz-frequency spinal cord stimulation produces intensity- and frequency-dependent inhibition of mechanical hypersensitivity in a rat model of neuropathic pain. Anesthesiology, 2013. 119(2): p. 422-32. 
68. Linderoth, B. and R.D. Foreman, Conventional and Novel Spinal Stimulation Algorithms: Hypothetical Mechanisms of Action and Comments on Outcomes. Neuromodulation, 2017. 20(6): p. 525-533.

69. Melzack, R. and P.D. Wall, Pain mechanisms: a new theory. Science, 1965. 150(3699): p. 9719.

70. North, R.B., et al., Spinal cord stimulation for chronic, intractable pain: superiority of "multichannel" devices. Pain, 1991. 44(2): p. 119-30.

71. Janssen, S.P., et al., Decreased intracellular GABA levels contribute to spinal cord stimulationinduced analgesia in rats suffering from painful peripheral neuropathy: the role of KCC2 and GABA(A) receptor-mediated inhibition. Neurochem Int, 2012. 60(1): p. 21-30.

72. Linderoth, B., et al., Gamma-aminobutyric acid is released in the dorsal horn by electrical spinal cord stimulation: an in vivo microdialysis study in the rat. Neurosurgery, 1994. 34(3): p. 484-8; discussion 488-9.

73. Stiller, C.O., et al., Release of gamma-aminobutyric acid in the dorsal horn and suppression of tactile allodynia by spinal cord stimulation in mononeuropathic rats. Neurosurgery, 1996. 39(2): p. 367-74; discussion 374-5.

74. Cui, J.G., et al., Spinal cord stimulation attenuates augmented dorsal horn release of excitatory amino acids in mononeuropathy via a GABAergic mechanism. Pain, 1997. 73(1): p. 87-95.

75. Cui, J.G., et al., Effect of spinal cord stimulation on tactile hypersensitivity in mononeuropathic rats is potentiated by simultaneous $G A B A(B)$ and adenosine receptor activation. Neurosci Lett, 1998. 247(2-3): p. 183-6.

76. Lind, G., et al., Baclofen-enhanced spinal cord stimulation and intrathecal baclofen alone for neuropathic pain: Long-term outcome of a pilot study. Eur J Pain, 2008. 12(1): p. 132-6.

77. Smits, H., M. van Kleef, and E.A. Joosten, Spinal cord stimulation of dorsal columns in a rat model of neuropathic pain: evidence for a segmental spinal mechanism of pain relief. Pain, 2012. 153(1): p. 177-83.

78. Tazawa, T., et al., Spinal cord stimulation modulates supraspinal centers of the descending antinociceptive system in rats with unilateral spinal nerve injury. Mol Pain, 2015. 11: p. 36.

79. Saade, N.E., et al., The role of the dorsolateral funiculi in the pain relieving effect of spinal cord stimulation: a study in a rat model of neuropathic pain. Exp Brain Res, 2015. 233(4): p. 104152.

80. Moens, M., et al., Spinal cord stimulation modulates cerebral function: an fMRI study. Neuroradiology, 2012. 54(12): p. 1399-407.

81. Stancak, A., et al., Functional magnetic resonance imaging of cerebral activation during spinal cord stimulation in failed back surgery syndrome patients. Eur J Pain, 2008. 12(2): p. 137-48.

82. Kiriakopoulos, E.T., et al., Functional magnetic resonance imaging: a potential tool for the evaluation of spinal cord stimulation: technical case report. Neurosurgery, 1997. 41(2): p. 5014.

83. Kulkarni, B., et al., Attention to pain localization and unpleasantness discriminates the functions of the medial and lateral pain systems. Eur J Neurosci, 2005. 21(11): p. 3133-42.

84. Basbaum, A.I., et al., Cellular and molecular mechanisms of pain. Cell, 2009. 139(2): p. 267-84.

85. Smits, H., et al., Experimental spinal cord stimulation and neuropathic pain: mechanism of action, technical aspects, and effectiveness. Pain Pract, 2013. 13(2): p. 154-68.

86. Verrills, P., C. Sinclair, and A. Barnard, A review of spinal cord stimulation systems for chronic pain. Journal of Pain Research, 2016. 9: p. 481-492.

87. Tracy Cameron, Safety and efficacy of spinal cord stimulation for the treatment of chronic pain: a 20-year literature review. Journal of Neurosurgery: Spine, 2004. 100(3): p. 254-267.

88. Levy, R.M., Progress in the Technology of Neuromodulation: The Emperor's New Clothes? Neuromodulation: Technology at the Neural Interface, 2013. 16(4): p. 285-291. 
89. Annemans, L., et al., Cost effectiveness of a novel $10 \mathrm{kHz}$ high-frequency spinal cord stimulation system in patients with failed back surgery syndrome (FBSS). J Long Term Eff Med Implants, 2014. 24(2-3): p. 173-83.

90. Taylor, R.S., et al., Predictors of pain relief following spinal cord stimulation in chronic back and leg pain and failed back surgery syndrome: a systematic review and meta-regression analysis. Pain Pract, 2014. 14(6): p. 489-505.

91. Huge, V., et al., Complex interaction of sensory and motor signs and symptoms in chronic CRPS. PLoS One, 2011. 6(4): p. e18775.

92. Guthmiller, K.B. and M. Varacallo, Complex Regional Pain Syndrome (CRPS), Reflex Sympathetic Dystrophy (RSD), in StatPearls. 2018: Treasure Island (FL).

93. Visnjevac, O., et al., A Comprehensive Outcome-Specific Review of the Use of Spinal Cord Stimulation for Complex Regional Pain Syndrome. Pain Pract, 2017. 17(4): p. 533-545.

94. Kumar, K., M. Abbas, and S. Rizvi, The use of spinal cord stimulation in pain management. Pain Manag, 2012. 2(2): p. 125-34.

95. Deer, T.R., et al., A prospective study of dorsal root ganglion stimulation for the relief of chronic pain. Neuromodulation, 2013. 16(1): p. 67-71; discussion 71-2.

96. Deer, T.R., et al., Dorsal root ganglion stimulation yielded higher treatment success rate for complex regional pain syndrome and causalgia at 3 and 12 months: a randomized comparative trial. Pain, 2017. 158(4): p. 669-681.

97. Huygen, F., et al., Stimulation of the L2-L3 Dorsal Root Ganglia Induces Effective Pain Relief in the Low Back. Pain Pract, 2018. 18(2): p. 205-213.

98. Liem, L., et al., One-year outcomes of spinal cord stimulation of the dorsal root ganglion in the treatment of chronic neuropathic pain. Neuromodulation, 2015. 18(1): p. 41-8; discussion 489.

99. Liem, L., et al., A multicenter, prospective trial to assess the safety and performance of the spinal modulation dorsal root ganglion neurostimulator system in the treatment of chronic pain. Neuromodulation, 2013. 16(5): p. 471-82; discussion 482.

100. Van Buyten, J.P., et al., High-frequency spinal cord stimulation for the treatment of chronic back pain patients: results of a prospective multicenter European clinical study. Neuromodulation, 2013. 16(1): p. 59-65; discussion 65-6.

101. Song, Z., B.A. Meyerson, and B. Linderoth, High-Frequency (1 kHz) Spinal Cord Stimulation-Is Pulse Shape Crucial for the Efficacy? A Pilot Study. Neuromodulation: Technology at the Neural Interface, 2015. 18(8): p. 714-720.

102. Al-Kaisy, A., et al., Sustained effectiveness of $10 \mathrm{kHz}$ high-frequency spinal cord stimulation for patients with chronic, low back pain: 24-month results of a prospective multicenter study. Pain Med, 2014. 15(3): p. 347-54.

103. Kriek, N., et al., Successful burst spinal cord stimulation in a CRPS patient with failed conventional stimulation. Neuromodulation, 2013. 16.

104. De Ridder, D., et al., Burst spinal cord stimulation: toward paresthesia-free pain suppression. Neurosurgery, 2010. 66(5): p. 986-90.

105. De Ridder, D. and S. Vanneste, Burst and Tonic Spinal Cord Stimulation: Different and Common Brain Mechanisms. Neuromodulation: Technology at the Neural Interface, 2016. 19(1): p. 4759.

106. Kriek, N., et al., Preferred frequencies and waveforms for spinal cord stimulation in patients with complex regional pain syndrome: A multicentre, double-blind, randomized and placebocontrolled crossover trial. Eur J Pain, 2016.

107. Perruchoud, C., et al., Analgesic efficacy of high-frequency spinal cord stimulation: a randomized double-blind placebo-controlled study. Neuromodulation, 2013. 16(4): p. 363-9; discussion 369. 
108. Al-Kaisy, A., et al., Prospective, Randomized, Sham-Control, Double Blind, Crossover Trial of Subthreshold Spinal Cord Stimulation at Various Kilohertz Frequencies in Subjects Suffering From Failed Back Surgery Syndrome (SCS Frequency Study). Neuromodulation, 2018. 21(5): p. 457-465.

109. Kapural, L., et al., Novel 10-kHz High-frequency Therapy (HF10 Therapy) Is Superior to Traditional Low-frequency Spinal Cord Stimulation for the Treatment of Chronic Back and Leg Pain: The SENZA-RCT Randomized Controlled Trial. Anesthesiology, 2015. 123(4): p. 851-60.

110. Lempka, S.F., et al., Computational analysis of kilohertz frequency spinal cord stimulation for chronic pain management. Anesthesiology, 2015. 122(6): p. 1362-76.

111. Kilgore, K.L. and N. Bhadra, Reversible nerve conduction block using kilohertz frequency alternating current. Neuromodulation, 2014. 17(3): p. 242-54; discussion 254-5.

112. Miller, J.P., et al., Parameters of Spinal Cord Stimulation and Their Role in Electrical Charge Delivery: A Review. Neuromodulation, 2016. 19(4): p. 373-84.

113. Al-Kaisy, A., et al., The use of 10-kilohertz spinal cord stimulation in a cohort of patients with chronic neuropathic limb pain refractory to medical management. Neuromodulation, 2015. 18(1): p. 18-23; discussion 23.

114. Kapural, L. and S. Harandi, Long-term efficacy of 1-1.2 kHz subthreshold spinal cord stimulation following failed traditional spinal cord stimulation: a retrospective case series. Reg Anesth Pain Med, 2019. 44(1): p. 107-110.

115. Thomson, S.J., et al., Effects of Rate on Analgesia in Kilohertz Frequency Spinal Cord Stimulation: Results of the PROCO Randomized Controlled Trial. Neuromodulation, 2018. 21(1): p. 67-76.

116. De Ridder, D., et al., Burst spinal cord stimulation for limb and back pain. World Neurosurg, 2013. 80(5): p. 642-649 e1.

117. Wu, G., et al., Early onset of spontaneous activity in uninjured $C$-fiber nociceptors after injury to neighboring nerve fibers. J Neurosci, 2001. 21(8): p. RC140.

118. Wu, G., et al., Degeneration of myelinated efferent fibers induces spontaneous activity in uninjured C-fiber afferents. J Neurosci, 2002. 22(17): p. 7746-53.

119. Amir, R., M. Michaelis, and M. Devor, Burst discharge in primary sensory neurons: triggered by subthreshold oscillations, maintained by depolarizing afterpotentials. J Neurosci, 2002. 22(3): p. 1187-98.

120. Emmers, R., Thalamic mechanisms that process a temporal pulse code for pain. Brain Res, 1976. 103(3): p. 425-41.

121. Radhakrishnan, V., et al., A comparison of the burst activity of lateral thalamic neurons in chronic pain and non-pain patients. Pain, 1999. 80(3): p. 567-75.

122. Lenz, F.A., et al., Neuronal activity in the region of the thalamic principal sensory nucleus (ventralis caudalis) in patients with pain following amputations. Neuroscience, 1998. 86(4): p. 1065-81.

123. Meuwissen, K.P.V., et al., Response to: Fundamental Differences in Burst Stimulation Waveform Design: Eliminating Confusion in the Marketplace. Neuromodulation, 2018. 21(7): p. 721-722.

124. Parker, J.L., et al., Compound action potentials recorded in the human spinal cord during neurostimulation for pain relief. Pain, 2012. 153(3): p. 593-601.

125. Tang, R., et al., Comparison of burst and tonic spinal cord stimulation on spinal neural processing in an animal model. Neuromodulation, 2014. 17(2): p. 143-51.

126. Crosby, N.D., et al., Stimulation parameters define the effectiveness of burst spinal cord stimulation in a rat model of neuropathic pain. Neuromodulation, 2015. 18(1): p. 1-8; discussion 8.

127. De Ridder, D. and S. Vanneste, Burst and Tonic Spinal Cord Stimulation: Different and Common Brain Mechanisms. Neuromodulation, 2016. 19(1): p. 47-59. 
128. de Vos, C.C., et al., Burst spinal cord stimulation evaluated in patients with failed back surgery syndrome and painful diabetic neuropathy. Neuromodulation, 2014. 17(2): p. 152-9.

129. Courtney, P., et al., Improved Pain Relief With Burst Spinal Cord Stimulation for Two Weeks in Patients Using Tonic Stimulation: Results From a Small Clinical Study. Neuromodulation, 2015. 18(5): p. 361-6.

130. De Ridder, D., et al., A 2-center comparative study on tonic versus burst spinal cord stimulation: amount of responders and amount of pain suppression. Clin J Pain, 2015. 31(5): p. 433-7.

131. Deer, T., et al., Success Using Neuromodulation With BURST (SUNBURST) Study: Results From a Prospective, Randomized Controlled Trial Using a Novel Burst Waveform. Neuromodulation, 2018. 21(1): p. 56-66.

132. Kriek, N., et al., Preferred frequencies and waveforms for spinal cord stimulation in patients with complex regional pain syndrome: A multicentre, double-blind, randomized and placebocontrolled crossover trial. Eur J Pain, 2017. 21(3): p. 507-519.

133. Kriek, N., et al., Spinal Cord Stimulation in Patients With Complex Regional Pain Syndrome: A Possible Target for Immunomodulation? Neuromodulation, 2018. 21(1): p. 77-86.

134. Van Havenbergh, T., et al., Spinal cord stimulation for the treatment of chronic back pain patients: $500-\mathrm{Hz}$ vs. $1000-\mathrm{Hz}$ burst stimulation. Neuromodulation, 2015. 18(1): p. 9-12; discussion 12.

135. Falowski, S.M., Fundamental Differences in Burst Stimulation Waveform Design: Eliminating Confusion in the Marketplace. Neuromodulation, 2018. 21(3): p. 320.

136. Crosby, N.D., et al., Burst and Tonic Spinal Cord Stimulation Differentially Activate GABAergic Mechanisms to Attenuate Pain in a Rat Model of Cervical Radiculopathy. IEEE Trans Biomed Eng, 2015. 62(6): p. 1604-13.

137. Cui, J.G., B. Linderoth, and B.A. Meyerson, Effects of spinal cord stimulation on touch-evoked allodynia involve GABAergic mechanisms. An experimental study in the mononeuropathic rat. Pain, 1996. 66(2-3): p. 287-95.

138. Mogil, J.S., Animal models of pain: progress and challenges. Nat Rev Neurosci, 2009. 10(4): p. 283-94.

139. Barrot, M., Tests and models of nociception and pain in rodents. Neuroscience, 2012. 211: p. 39-50.

140. Janssen, S.P., et al., Differential GABAergic disinhibition during the development of painful peripheral neuropathy. Neuroscience, 2011. 184: p. 183-94.

141. Truin, M., et al., Increased efficacy of early spinal cord stimulation in an animal model of neuropathic pain. Eur J Pain, 2011. 15(2): p. 111-7.

142. Truin, M., et al., Successful pain relief in non-responders to spinal cord stimulation: The combined use of ketamine and spinal cord stimulation. European Journal of Pain, 2011. 15(10).

143. Smits, H., et al., Effect of spinal cord stimulation in an animal model of neuropathic pain relates to degree of tactile "allodynia". Neuroscience, 2006. 143(2): p. 541-6.

144. Gong, W.Y., L.M. Johanek, and K.A. Sluka, A Comparison of the Effects of Burst and Tonic Spinal Cord Stimulation on Hyperalgesia and Physical Activity in an Animal Model of Neuropathic Pain. Anesth Analg, 2016.

145. Vierck, C.J. and R.P. Yezierski, Comparison of operant escape and reflex tests of nociceptive sensitivity. Neurosci Biobehav Rev, 2015. 51: p. 223-42.

146. Vierck, C.J., P.T. Hansson, and R.P. Yezierski, Clinical and pre-clinical pain assessment: are we measuring the same thing? Pain, 2008. 135(1-2): p. 7-10.

147. Sato, K.L., et al., Spinal cord stimulation reduces hypersensitivity through activation of opioid receptors in a frequency-dependent manner. Eur J Pain, 2013. 17(4): p. 551-61.

148. Meuwissen, K.P.V., et al., Burst Spinal Cord Stimulation in Peripherally Injured Chronic Neuropathic Rats: A Delayed Effect. Pain Pract, 2018. 
149. Meuwissen, K.P.V., et al., Conventional-SCS vs. Burst-SCS and the Behavioral Effect on Mechanical Hypersensitivity in a Rat Model of Chronic Neuropathic Pain: Effect of Amplitude. Neuromodulation, 2018. 21(1): p. 19-30.

150. van Beek, M., et al., Spinal cord stimulation in experimental chronic painful diabetic polyneuropathy: Delayed effect of High-frequency stimulation. Eur J Pain, 2016.

151. Weiss, B. and V.G. Laties, Fractional escape and avoidance on a titration schedule. Science, 1958. 128(3338): p. 1575-6.

152. Martin, T.J., et al., Effects of laparotomy on spontaneous exploratory activity and conditioned operant responding in the rat: a model for postoperative pain. Anesthesiology, 2004. 101(1): p. 191-203.

153. Neubert, J.K., et al., Use of a novel thermal operant behavioral assay for characterization of orofacial pain sensitivity. Pain, 2005. 116(3): p. 386-95.

154. Thut, P.D., et al., An operant conditioning model to assess changes in feeding behavior associated with temporomandibular joint inflammation in the rat. J Orofac Pain, 2007. 21(1): p. 7-18.

155. Martin, T.J. and E. Ewan, Chronic pain alters drug self-administration: implications for addiction and pain mechanisms. Exp Clin Psychopharmacol, 2008. 16(5): p. 357-66.

156. Sufka, K.J., Conditioned place preference paradigm: a novel approach for analgesic drug assessment against chronic pain. Pain, 1994. 58(3): p. 355-66.

157. Johansen, J.P., H.L. Fields, and B.H. Manning, The affective component of pain in rodents: direct evidence for a contribution of the anterior cingulate cortex. Proc Natl Acad Sci U S A, 2001. 98(14): p. 8077-82.

158. LaBuda, C.J. and P.N. Fuchs, A behavioral test paradigm to measure the aversive quality of inflammatory and neuropathic pain in rats. Exp Neurol, 2000. 163(2): p. 490-4.

159. Harte, S.E., et al., Mechanical Conflict System: A Novel Operant Method for the Assessment of Nociceptive Behavior. PLoS One, 2016. 11(2): p. e0150164.

160. Coy-Laboratory, Coy Mechanical Conflict-Avoidance System for rats (Harte, Morrow method): Reference Manual. 2013.

161. Lau, D., et al., Herpes simplex virus vector-mediated expression of interleukin-10 reduces below-level central neuropathic pain after spinal cord injury. Neurorehabil Neural Repair, 2012. 26(7): p. 889-97.

162. De Ridder, D., S. Perera, and S. Vanneste, Are $10 \mathrm{kHz}$ Stimulation and Burst Stimulation Fundamentally the Same? Neuromodulation, 2017. 20(7): p. 650-653.

163. Zhang, T.C., J.J. Janik, and W.M. Grill, Modeling effects of spinal cord stimulation on widedynamic range dorsal horn neurons: influence of stimulation frequency and GABAergic inhibition. J Neurophysiol, 2014. 112(3): p. 552-67. 



\section{CHAPTER 2}

Conventional-SCS vs. Burst-SCS and the Behavioral Effect on Mechanical Hypersensitivity in a Rat Model of Chronic Neuropathic Pain: Effect of Amplitude.

Koen P.V. Meuwissen, Jianwen Wendy Gu, Tianhe C. Zhang, Elbert A.J. Joosten.

Neuromodulation: Technology at the Neural Interface 2018; 21: 19-30.

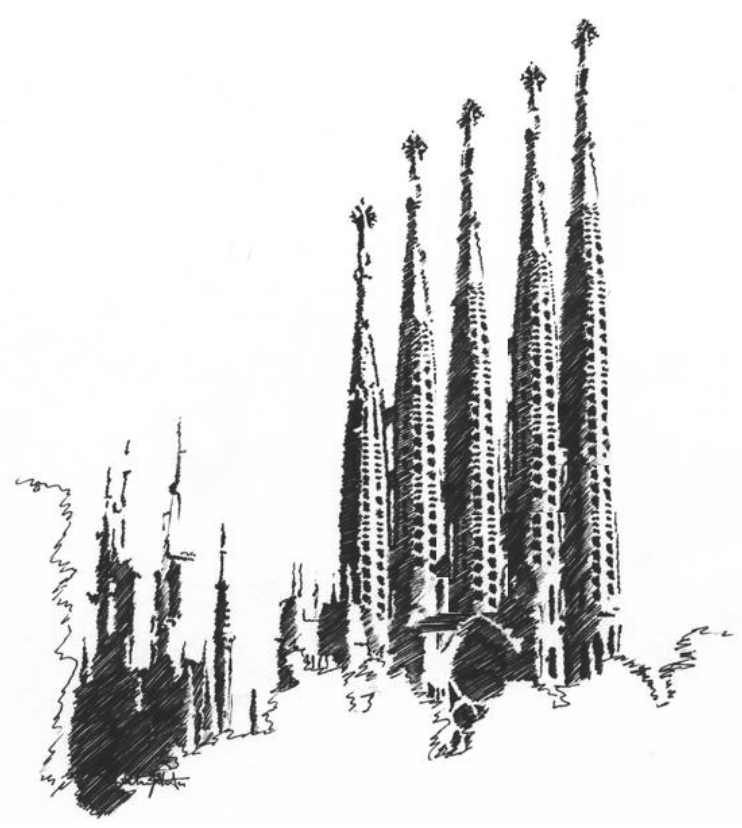




\begin{abstract}
OBJECTIVE: Various spinal cord stimulation (SCS) modes are used in the treatment of chronic neuropathic pain disorders. Conventional (Con) and Burst-SCS are hypothesized to exert analgesic effects through different stimulation-induced mechanisms. Preclinical electrophysiological findings suggest that stimulation intensity is correlated with the effectiveness of Burst-SCS [1]. Therefore, we aimed to investigate the relation between amplitude (charge per second) and behavioral effects in a rat model of chronic neuropathic pain, for both Con-SCS and biphasic Burst-SCS.
\end{abstract}

METHODS: Animals ( $n=12$ rats) received a unilateral partial sciatic nerve ligation, after which they were implanted with quadripolar electrodes in the epidural space at thoracic level 13. Mechanical hypersensitivity was assessed using paw withdrawal thresholds (WTs) to von Frey monofilaments, at various SCS intensities (amplitudes) and multiple time points during 60 minutes of stimulation and 30 minutes post stimulation.

RESULTS: Increasing amplitude was shown to improve the efficacy of Con-SCS, whereas the efficacy of Burst-SCS showed a non-monotonic relation with amplitude. Con-SCS at 66\% MT $(n=5)$ and Burst-SCS at 50\% MT (n=6) were found to be equally effective in normalizing mechanical hypersensitivity. However, in the assessed time period Burst-SCS required significantly more mean charge per second to do so $(p<0.01)$. When applied at comparable mean charge per second, Con-SCS resulted in a superior behavioral outcome $(p<0.01)$, compared to Burst-SCS.

CONCLUSIONS: Biphasic Burst-SCS requires significantly more mean charge per second in order to achieve similar pain relief, as compared with Con-SCS, in an experimental model of chronic neuropathic pain. 


\section{Introduction}

Over the last four decades, spinal cord stimulation (SCS) has become a safe and effective therapeutic tool for the treatment of various neuropathic pain disorders that fail to respond to pharmacological intervention [2-4]. Conventional (Con-) SCS delivers tonic pulses of electrical stimulation to the dorsal columns of the spinal cord, typically using frequencies of $40-60 \mathrm{~Hz}[5,6]$ and pulse widths of $200-450 \mu \mathrm{Sec}[7,8]$. However, despite considerable improvements, the therapeutic effect of Con-SCS remains moderate, providing approximately $50 \%$ pain relief for $50 \%$ of patients $[9,10]$. Consequently, in an effort to improve the efficacy of SCS, several alternative SCS-modes have been developed, including high frequency spinal cord stimulation (HF-SCS) (>500 Hz; most often $10 \mathrm{kHz}$ ) [11-13] and burst spinal cord stimulation (Burst-SCS) $[14,15]$. Unlike Con-SCS, Burst-SCS and HF-SCS provide pain relief without eliciting paresthesia in the painful area. However, the mechanisms underlying the analgesic effects of the various SCS-modes continue to remain unclear. Preclinical studies suggest that Burst-SCS does not act via spinal GABAergic mechanisms in which Con-SCS acts, and might rely on different biochemical pathways [16]. Additional electrophysiological preclinical studies indicate that Burst-SCS can be optimized by adjusting stimulation parameters closely related to the energy delivered to the spinal cord, such as amplitude and pulse width, with reports that the energy delivered to the spinal cord was found to be correlated with a reduction of 'wide dynamic range neuronal' firing in the dorsal horn following noxious stimuli [1]. Other studies have shown that BurstSCS and Con-SCS have a different effect on the suppression of lumbosacral neural responses when applied with a high-intensity ( $90 \% \mathrm{MT})$ when compared with standard-intensity $(60 \%$ MT) [17]. To elucidate the relation between current delivery and the therapeutic effects of Burst-SCS, we studied the relation between amplitude and mean current delivered (charge per second), and the behavioral effect on mechanical hypersensitivity in a rat model of chronic neuropathic pain. 


\section{Methods}

\section{Ethics Statement}

All experiments were performed in accordance with the European Directive for the Protection of Vertebrate Animals Used for Experimental and Other Scientific Purposes (86/609/EU). The protocol was approved by the Animal Research Committee of the Maastricht University Medical Centre (DEC-protocol 2014-086).

\section{Animals}

All experiments were performed using male Sprague Dawley rats $(n=12)$, which were classified as young-adult (5 weeks of age) at the start of the experiment (150-200 g). Animals were housed in groups of 2 in filter-top polycarbonate cages situated within a climate-controlled vivarium $\left(21 \pm 1^{\circ} \mathrm{C}\right)$ with relative humidity $(55 \pm 15 \%)$, artificial lighting (12:12 light/dark cycle). Distilled water and rodent food were available ad libitum. The vivarium was equipped with a mobile radio, continuously producing background music at 45 decibel, in order to desensitize the animals for translocation and experimenter related noise. All procedures were conducted between 09:00 and 16:00 hours.

\section{Neuropathic Pain model}

A unilateral ligation of the left sciatic nerve was performed as described by Seltzer et al. 1990 [18] according to the standard protocol used in our institution $[19,20]$. Animals were anesthetized with 3-5\% isoflurane (Abbott Laboratories Ltd., Kent, U.K.) and air enriched with $100 \%$ oxygen at a constant flow rate of $250 \mathrm{ml} / \mathrm{min}$. Body temperature was maintained at $37.5^{\circ} \mathrm{C}$ using an automatic heating pad. The sciatic nerve from the left hind paw was exposed by blunt dissection. Upon discovery the nerve was carefully freed from surrounding connective tissue and partially (approximately $1 / 3$ ) ligated by an $8 / 0$ non-absorbable silk suture with a reverse cutting micro needle. Post ligation, the wound was closed with a $4 / 0$ silk suture, and the development of tactile hypersensitivity (mechanical allodynia) was monitored over the following 14 days. The presence of mechanical hypersensitivity was confirmed at 14 days post-surgery, only if the log (50\% withdrawal threshold (WT)) was decreased by 0.2 units compared to baseline (day 0) [21]. 


\section{Assessment of mechanical hypersensitivity (von Frey assay)}

Mechanical hypersensitivity was assessed using von Frey filaments. Von Frey testing was always conducted in the same room, which was isolated from all external sound and light sources, maintained under controlled temperature $\left(21 \pm 1^{\circ} \mathrm{C}\right)$ and relative humidity $(55 \pm 15 \%)$. Prior to testing, rats were placed in the behavioral set-up and were given $15 \mathrm{~min}$. to acclimate to the new surroundings. The test-room was equipped with a mobile radio, continuously producing background music at 45 decibels. WT to von Frey filaments was assessed using the 'up-down method' [22] whereby von Frey filaments with approximately linearly incrementing stiffness on a logarithmic scale (bending forces 0.6, 1.2, 2.0, 3.6, 5.5, $8.5,15.1 \mathrm{~g}$ and $28.84 \mathrm{~g}$ ) were applied to the plantar surface of the hind paws of the rats for 5 seconds. Following a negative response (the hind paw was not withdrawn), the next filament with greater bending force was applied, whereas a positive response (the hind paw was withdrawn) was followed with application of a previous filament with lower bending force. After completion of a sequence of six consecutive responses the $50 \%$ WT was calculated [22]. A predetermined cut-off value was set at $28.84 \mathrm{~g}$. For statistical analysis the $50 \%$ WTs were logarithmically transformed to yield a linear scale.

\section{Implantation of spinal cord stimulation device}

The implantation of the SCS device was performed according to the standard protocol used in our institution [20, 23-25], and is identical to the method originally developed at the Karolinska Institute [26]. In short, a small laminectomy was made at level Thoracic 13 (Th13), after which the spinal cord was exposed by use of a surgical rotary tool. A custommade cylindrical 4-contact lead $(0.72 \mathrm{~mm}$ diameter; Boston Scientific Neuromodulation, Valencia, CA, USA) was inserted in the epidural space, in the caudal direction. Electrode configuration was set at alternating cathode and anode settings (rostral to caudal: + - + -). Subsequently, the electrode was secured to a spinous process with tissue adhesive (Histoacryl ${ }^{\circledR}$, B Braun Medical BV, Oss, the Netherlands) to prevent electrode migration. The electrode wires were tunneled subcutaneously to the animal's neck and the stimulatorconnectors were attached to the skin. After implantation of the electrodes, the rats were given 3 days for recovery prior to the initiation of SCS.

\section{Spinal cord stimulation}

For stimulation of the dorsal columns, an A-M systems stimulator (MultiStim: Programmable 8-Channel Stimulator (Model 3800) $220 \mathrm{~V} / 50 \mathrm{~Hz}$ ) fitted with an additional stimulus Isolator (Model 3820 for A-M Systems MultiStim). For all experiments, the 
stimulator was set to deliver constant current biphasic stimulation for both the Conventional and Burst modes. Con-SCS motor threshold (MT) was determined at the following settings: pulse width of $200 \mu \mathrm{S}$ and frequency of $2 \mathrm{~Hz}$. Burst-SCS MT was determined using a pulse width of $1000 \mu \mathrm{S}$, where five pulses (449 Hz intraburst frequency) were administered at an inter-burst frequency of $2 \mathrm{~Hz}$. The amplitude was gradually increased until symmetrical contractions of the lower trunk and/or hind limbs were visually observed or perceived by hand. Then, either a Con-SCS-modes (frequency $50 \mathrm{~Hz}$, pulse width $200 \mu \mathrm{S}$ ) [20], or a Burst-SCS modes (inter-burst frequency $40 \mathrm{~Hz}, 5$ pulses at $449 \mathrm{~Hz}$ intraburst, pulse width $1000 \mu \mathrm{S}$ ) (Fig.1), was applied for 60 minutes [20, 27]. In all experiments, the WT to von Frey filaments was assessed before the start of SCS treatment, at $15^{\prime}, 30^{\prime}, 45^{\prime}$ and $60^{\prime}$ min after stimulation was turned on, and at 30 min after stimulation was turned off (90 min after the start of SCS). Stimulation was applied for 60 minutes per day on three consecutive days. A different amplitude ( $66 \%, 50 \%$ or $33 \% \mathrm{MT})$ was used each day, and the order was randomized. The investigator was blinded to the stimulation condition during behavioral testing. Furthermore, after the experiment, 3 animals of the Burst-group as well as 3 animals of the Con-SCS group were administered SCS at an amplitude in order to provide a comparable delivery of current (Coulomb per second). Mean current was calculated, as charge per second, by use of the following formula: $I_{\text {mean }}=$ amplitude $x$ pulse width $x$ frequency.

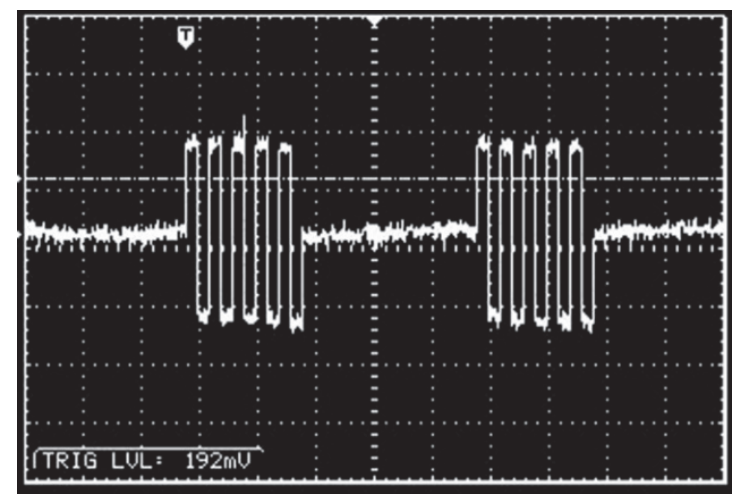

Fig.1: Constant current biphasic burst mode $(\mu \mathrm{A})$ : 1-ms biphasic spikes with a 1.1-ms spike interval (449- $\mathrm{Hz}$ spike mode) at $40 \mathrm{~Hz}(40-\mathrm{Hz}$ burst mode). Stimulation delivered by the A-M systems stimulator (MultiStim: Programmable 8-Channel Stimulator (Model 3800). 


\section{Timeline of experiments}

After acclimatization to the vivarium, 12 animals underwent a partial sciatic nerve ligation (PSNL) (as described in section 2.3). During the subsequent 14-day observation period, animals received Von Frey measurements in order to assess the development of tactile hypersensitivity (see section 2.3). All 12 animals successfully developed tactile hypersensitivity and received implantation of an SCS-electrode at day post ligation (DPL) 14, as described in section 2.5. Animals for which the SCS-electrode was successfully implanted $(n=11)$ were given two days to recover from the surgery before the Motor-Threshold (MT) was assessed at DPL17. All implanted animals showed a robust MT ( $n=11)$. At DPL18, 19, 20 animals received SCS for 60 minutes (Fig.2). Mechanical hypersensitivity was assessed based on WT to von Frey filaments, at 15, 30, 45 and 60 minutes of stimulation, and 30 minutes post stimulation.

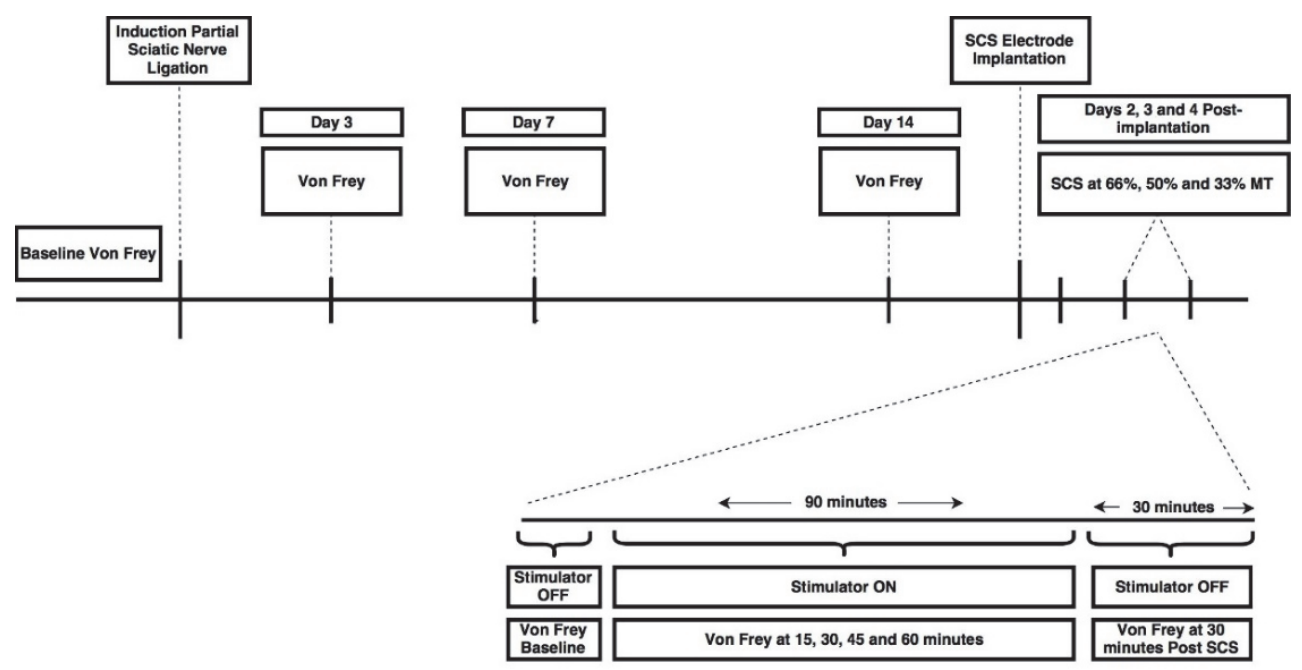

Fig.2: Timeline of experiments.

\section{Data-analysis}

The WTs to von Frey filaments are presented as mean \pm standard error of the mean (SEM). For statistical analysis von Frey data were logarithmically transformed to account for Weber's Law [28]. For analysis of differences in the withdrawal thresholds over time, a oneway repeated measures ANOVA was conducted and followed by a Dunnett's multiple comparisons test. For the analysis of differences in the withdrawal thresholds between 
groups within stimulation modes (Burst- or Con-SCS), a two-way repeated measures ANOVA was conducted and followed by Tukey's multiple comparisons test for each individual time point. For the analysis of differences in the withdrawal thresholds between groups and stimulation modes a two-way ANOVA was applied, followed by Tukey's multiple comparisons test. All statistical analyses were performed with $\alpha=0.05$ using IBM SPSS statistics 23.

\section{Results}

\section{General observations}

No significant differences were observed between ipsilateral pre-SCS withdrawal thresholds (WTs) between amplitude groups, within modes of stimulation (Con-SCS groups: $p=0.7308$; Burst-SCS groups: $p=0.5920$ ) or between modes of stimulation (Con-SCS vs. Burst-SCS groups: $p=0.4227$ ). The order of amplitudes (percentages of MT) had no significant effects on the Con-SCS group ( $p=0.7770)$, the Burst-SCS group $(p=0.8423)$, or the Burst-SCS groups compared with Con-SCS groups $(p=0.2020)$. The average motor thresholds for Con-CS (120 $\pm 3.8 \mu \mathrm{A})$ and Burst-SCS $(49 \pm 2.7 \mu \mathrm{A})$ were statistically different $(\mathrm{p}<0.007)$ (Fig.3).

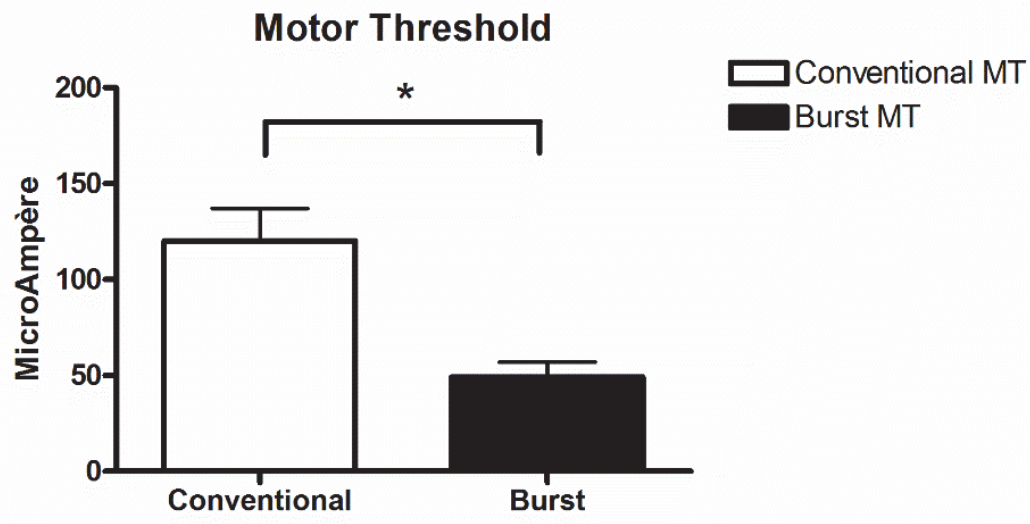

Fig.3: Motor threshold (MT) assessed by means of Conventional and Burst stimulation. Con-SCS MT was determined at the following settings: pulse width of $200 \mu \mathrm{S}$ administered at a frequency of $2 \mathrm{~Hz}$. Burst-SCS MT was determined at the following settings: pulse width of $1000 \mu \mathrm{S}$, five pulses $(500 \mathrm{~Hz}$ intraburst frequency) administered at an interburst-frequency of $2 \mathrm{~Hz}$. (* $p<0.05$ for Con-MTs versus Burst-MTs). 


\section{Development of tactile hypersensitivity}

Pre-nerve injury (baseline) mean withdrawal threshold of the ipsilateral hind paws were comparable to the contralateral hind paws (ipsilateral 11.2 $\pm 0.3 \mathrm{~g}$ vs contralateral $10.1 \pm$ $0.4 \mathrm{~g} ; p=0.3777$ ) in all animals (Fig. 4). Fourteen days after the partial ligation of the sciatic nerve, the mean withdrawal threshold of the ipsilateral paw $(1.4 \pm 0.02 \mathrm{~g})$ was significantly lower than baseline $(p<0.001)$. All 12 animals qualified as hypersensitive to mechanical stimulation by von Frey filaments (see Methods section 2.3) and were therefore included for further analysis (Fig.2+4). No significant effect of the ipsilateral ligation on contralateral paw withdrawal thresholds could be detected $(10.1 \pm 0.4 \mathrm{~g}$ pre-surgery vs $12.5 \pm 0.5 \mathrm{~g}$ post-surgery; $p=0.1963$ ). The implantation of the SCS electrode at DPL14 did not affect the withdrawal thresholds of the ipsilateral hind paws (DPL14; $1.4 \pm 0.02 \mathrm{~g}$ vs DPL17; $1.3 \pm 0.05 \mathrm{~g} ; p=0.4227$ ).

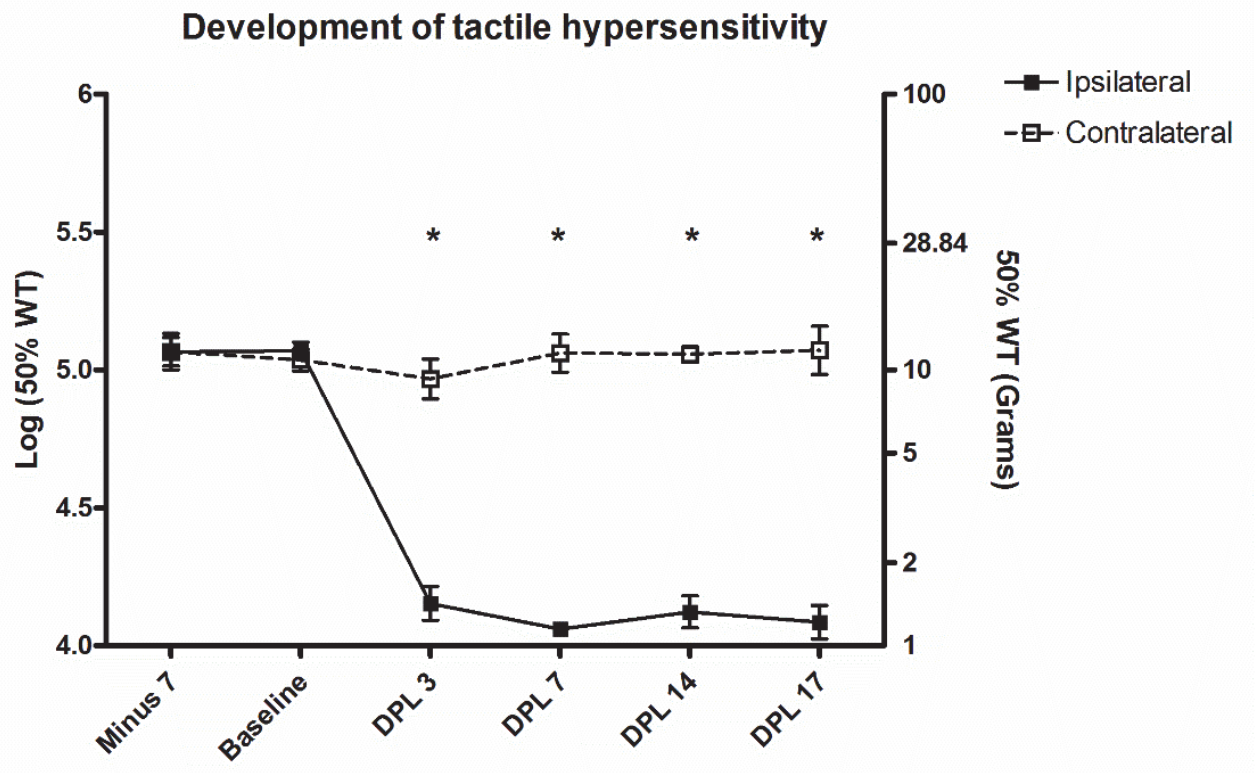

Fig.4: Development of tactile hypersensitivity, based on paw withdrawal threshold s (WTs) based on sensitivity to von Frey filaments, after unilateral sciatic nerve ligation in adult rat $(n=12)$. ( $^{*} p<0.05$ for ipsilateral versus contralateral WT's). 


\section{Within modes of stimulation}

Stimulation was delivered at $66 \%, 50 \%$ and $33 \%$ of MT for the Con-SCS group $(n=5)$ and Burst-SCS group $(n=6)$. In order to assess differences in the withdrawal thresholds over time a one-way repeated measures ANOVA was applied for each individual amplitude group within one SCS mode (Conventional or Burst SCS). After 15 minutes of SCS in the Con-SCS groups, only the $66 \% \mathrm{MT}$ group significantly differed from baseline $(2.0 \pm 0.3 \mathrm{~g}$ vs $8.4 \pm 1.5 \mathrm{~g}$; $p=0.0031$ ) (Fig.5A). Thirty minutes of SCS significantly increased WTs compared to baseline for both the $66 \%$ MT and $50 \%$ MT groups ( $50 \% \mathrm{MT} ; 1.8 \pm 0.3 \mathrm{~g}$ vs $7.2 \pm 1.2 \mathrm{~g} ; p=0.002,66 \%$ MT; $2.0 \pm 0.3 \mathrm{~g}$ vs $11.4 \pm 0 \mathrm{~g} ; p=0.0013$ ), and both groups remained significantly different (from baseline) until $60 \mathrm{~min}$ of SCS (see Table 1). This was corroborated by a significant main effect of time for Con-SCS applied at 50\% MT ( $p=0.0003)$, and 66\% MT $(p<0.0001)$. Post SCS, 30 minutes after the stimulator was turned off, only the $66 \%$ MT group was still significantly different from baseline $(2.0 \pm 0.3 \mathrm{~g}$ vs $6.0 \pm 0.6 \mathrm{~g} ; p=0.008)$. The $33 \% \mathrm{MT}$ group did not demonstrate an overall analgesic effect and was only significantly different from baseline at 45 minutes of SCS $(1.6 \pm 0.2 \mathrm{~g}$ vs $2.9 \pm 0.2 \mathrm{~g} ; p=0.0125)$. This was consistent with absence of a (significant) main effect of time for Con-SCS applied at 33\% of the MT ( $p=$ 0.1025) (Fig.5A).

After 15 minutes of SCS in the Burst-SCS groups, none of the groups significantly differed from baseline. In contrast, all three groups significantly differed from baseline after 30 minutes of SCS (33\% MT; $1.5 \pm 0.2 \mathrm{~g}$ vs $5.0 \pm 0.6 \mathrm{~g} ; p=0.0056,50 \% \mathrm{MT} ; 1.4 \pm 0.2 \mathrm{~g}$ vs $10.1 \pm$ $0.9 \mathrm{~g} ; p=0.0001,66 \% \mathrm{MT} ; 1.2 \pm 0.2 \mathrm{~g}$ vs $6.3 \pm 0.7 \mathrm{~g} ; p=0.0025)$, and remained significantly different (from baseline) until $60 \mathrm{~min}$ of SCS (see Table 1). This was in line with significant main effect of time for Burst-SCS applied at 33\%MT ( $p<0.0001), 50 \% \mathrm{MT}(p<0.0001)$, and $66 \%$ MT $(p<0.0001)$. (Fig.5B). Post SCS, 30 minutes after the stimulator was turned off, both the $66 \% \mathrm{MT}$ and $50 \% \mathrm{MT}$ group were still significantly different from baseline $(50 \%$ $\mathrm{MT} ; 1.4 \pm 0.2 \mathrm{~g}$ vs $3.1 \pm 0.4 \mathrm{~g} ; p=0.0074,66 \% \mathrm{MT} ; 1.2 \pm 0.2 \mathrm{~g}$ vs $2.4 \pm 0.3 \mathrm{~g} ; p=0.0121$ ). 

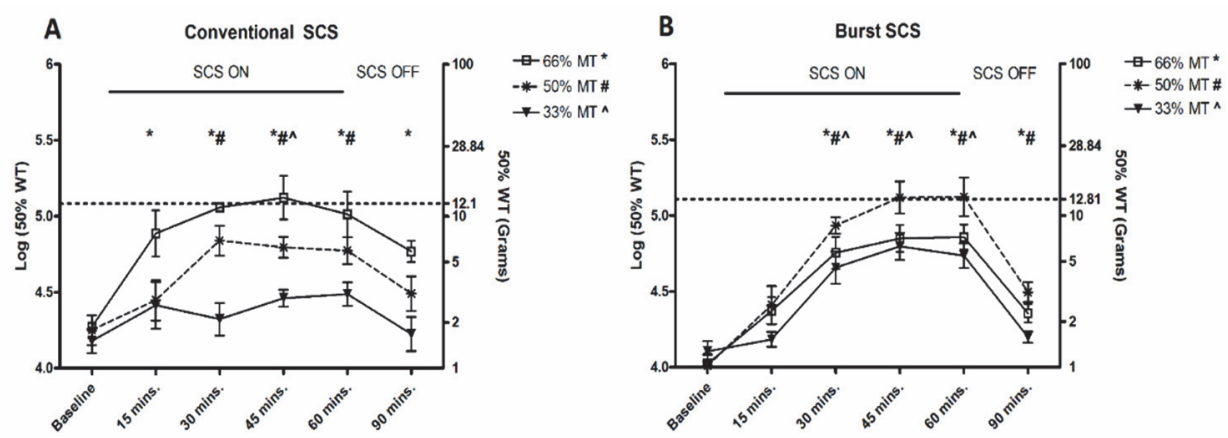

Fig 5. $(A+B)$ : The effect of Con-SCS $(n=5)$ and Burst-SCS $(n=6)$ on the WTs based on sensitivity to von Frey filaments. WTs were assessed at 15, 30, 45, and 60 mins. of stimulation and 30 mins. post stimulation. The dotted line represents the average WT baseline prior to sciatic nerve ligation. $\left(^{*} p<\right.$ 0.05 for $66 \%$ MT time points compared to pre-SCS baseline WTs, \# $p<0.05$ for $50 \%$ MT time points compared to pre-SCS baseline WTs, ^ $\mathrm{p}<0.05$ for $33 \% \mathrm{MT}$ time points compared to pre-SCS baseline WTs).

\section{Maximum effect of stimulation modes}

Con-SCS at $66 \%$ MT amplitude stimulation resulted in the most optimal behavioral outcome for Con-SCS, significantly increasing ipsilateral WTs from $2.0 \pm 0.3 \mathrm{~g}$ (pre-SCS baseline withdrawal thresholds) to $14.1 \pm 2.6 \mathrm{~g}$ after 45 minutes of SCS ( $p=0.0024)$. WTs of $66 \%$ MT Con-SCS after 45 minutes also significantly differed from the WTs of Con-SCS at $50 \%$ and $33 \%$ MT after 45 minutes of SCS (two-way repeated measures ANOVA, followed by Tukey's multiple comparisons test: $66 \%$ vs $50 \% ; p=0.0015,66 \%$ vs $33 \% ; p<0.0001$ ) (Fig.5A). BurstSCS at 50\% MT resulted in the most optimal behavioral outcome for Burst-SCS, significantly increasing ipsilateral WTs from 1.4 $\pm 0.2 \mathrm{~g}$ (pre-SCS baseline withdrawal threshold) to $14.8 \pm$ $2.6 \mathrm{~g}$ after 60 minutes of SCS $(p=0.0013)$, reaching pre-surgery WTs. WTs of 50\% MT BurstSCS after 60 minutes was also significantly different from the WTs of Burst-SCS at $66 \%$ and $33 \% \mathrm{MT}$ after 60 minutes of SCS (two-way repeated measures ANOVA, followed by Tukey's multiple comparisons test:50\% vs 66\%; $p<0.0123$, 50\% vs 33\%; $p<0.0001$ ) (Fig. 5B). Interestingly, Burst- SCS at 50\% MT after 60 minutes was not significantly different from Con-SCS at $66 \%$ MT after 45 minutes of SCS (Burst $14.8 \pm 2.6 \mathrm{~g}$ vs Con $14.1 \pm 2.6 \mathrm{~g} ; p=0,9744$ ), which indicates that Burst-SCS and Con-SCS are equally effective in normalizing mechanical hypersensitivity. 


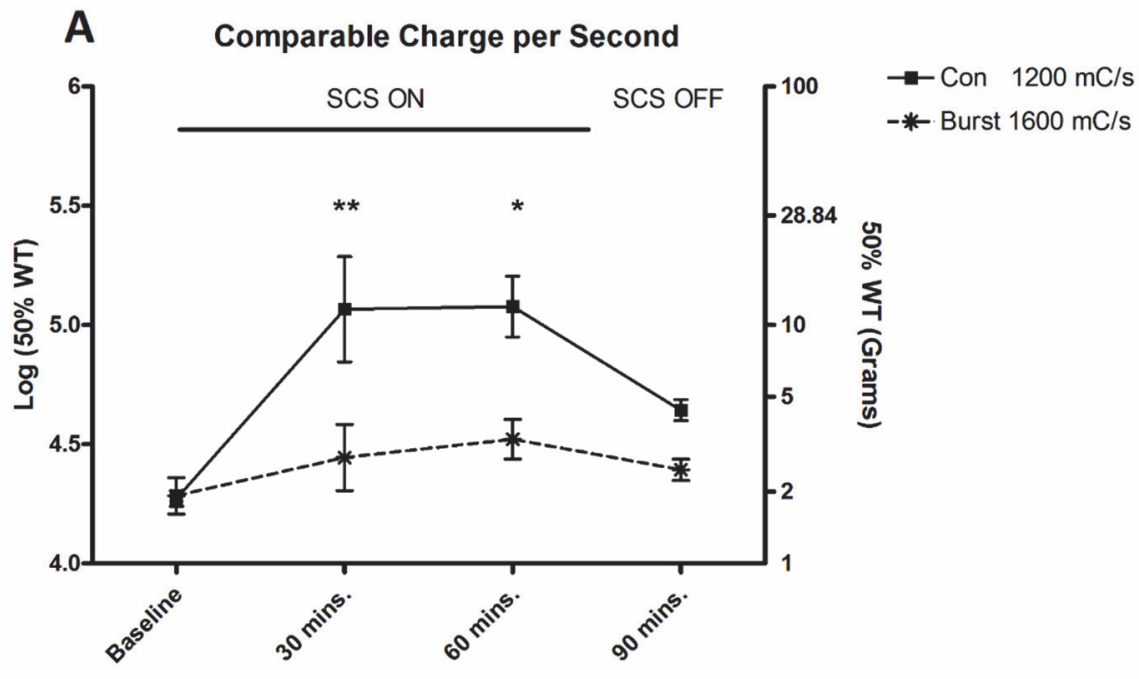

\section{B Comparable charge per second}

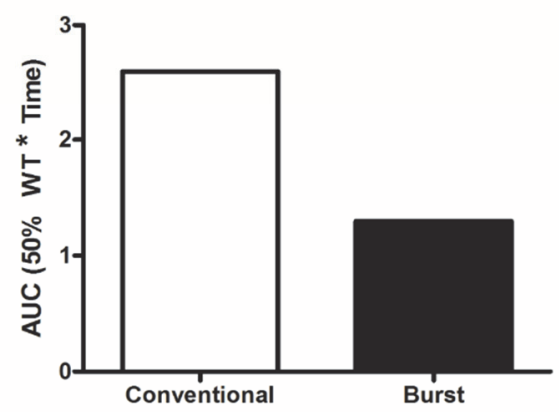

Fig. 6A: The effect of Con-SCS and Burst-SCS on WTs, at comparable charge per second (Con: 1200 $\mathrm{mC} / \mathrm{s}$; Burst: $1600 \mathrm{mC} / \mathrm{s}),(\mathrm{n}=3$ per group). WTs were assessed at 30, and 60 mins. of stimulation and 30 mins. post stimulation. ( ${ }^{*} p<0.05$ for Con-SCS WTs versus Burst-SCS WTs per time point, ${ }^{* *} p<0.01$ for Con-SCS WTs versus Burst-SCS WTs per time point). B: Area under the curve (AUC) for WTs of ConSCS and Burst-SCS, at comparable mean charge per second (Con: $1200 \mathrm{mC} / \mathrm{s}$, Burst: $1600 \mathrm{mC} / \mathrm{s}),(\mathrm{n}=$ 3 per group). 


\section{Between modes of stimulation}

WTs of the Conventional 66\% MT group significantly differed from the Burst $66 \%$ MT group $(p<0.001)$. Tukey's multiple comparisons test for specific time points shows that the Conventional 66\% MT group and Burst 66\% MT group significantly differ after 15 minutes of SCS ( $8.4 \pm 1.5 \mathrm{~g}$ vs $2.4 \pm 0.2 \mathrm{~g} ; p<0.0001)$, after 30 minutes of SCS $(11.4 \pm 0 \mathrm{~g}$ vs $6.3 \pm 0.7 \mathrm{~g}$; $p=0.0397)$, and 30 minutes post SCS $(6.0 \pm 0.6 \mathrm{~g}$ vs $2.4 \pm 0.2 \mathrm{~g} p=0.0003)$. Overall the WTs of the Conventional $50 \%$ MT group did not significantly differ from the Burst $50 \%$ MT group ( $p=0.2893$ ). WTs of the Conventional $33 \% \mathrm{MT}$ group significantly differed overall from the Burst 33\% MT group $(p<0.001)$. Tukey's multiple comparisons test for specific time points shows that the Conventional 33\% MT group and Burst 33\% MT group significantly differed after 30 minutes of SCS ( $2.4 \pm 0.7 \mathrm{~g}$ vs $4.9 \pm 0.6 \mathrm{~g} ; p=2710.0028)$, and after 45 minutes of SCS $(2.9 \pm 0.2 \mathrm{~g}$ vs $6.2 \pm 0.5 \mathrm{~g} ; p=0.0082)$. The most optimal amplitudes for both modes, Conventional 66\% MT and Burst 50\% MT group did not significantly differ overall ( $p=$ 0.1203). However, Tukey's multiple comparisons test for specific time points showed that WTs of the Conventional 66\% MT group significantly differed from the Burst $50 \%$ MT group after 15 minutes of stimulation ( $8.4 \pm 1.5 \mathrm{~g}$ vs $3.2 \pm 0.6 \mathrm{~g} ; p<0.0002)$, and 30 minutes post stimulation $(6.0 \pm 0.6 \mathrm{~g}$ vs $3.0 \pm 0.4 \mathrm{~g} ; p<0.0277)$. This suggests a slower wash-in of the analgesic effect for Burst-SCS. Interestingly, when Burst-SCS $(n=3)$ and Con-SCS $(n=3)$ were applied at comparable charge per second (Con-SCS: $1200 \mathrm{mC} / \mathrm{s}$, Burst-SCS: $1600 \mathrm{mC} / \mathrm{s}$ ), ConSCS resulted in a superior behavioral outcome (30 minutes: Con-SCS:14.9 $\pm 5.5 \mathrm{~g}$ vs BurstSCS: $3.1 \pm 0.7 g ; p<0.01,60$ minutes: Con-SCS:13.0 \pm 3.0 g vs Burst-SCS: $3.4 \pm 0.5 g ; p<0.05$ ) (Fig. 6A).

Mean current was calculated, as charge per second, by use of the following formula: $I_{\text {mean }}$ $=$ amplitude $\mathrm{x}$ pulse width $\mathrm{x}$ frequency. Mean charge per second for Con-SCS was significantly lower as compared with Burst-SCS for 33\% MT ( $p<0.01), 50 \%$ MT $(p<0.01)$, and $66 \% \mathrm{MT}(p<0.01)$ (Fig. 7). 


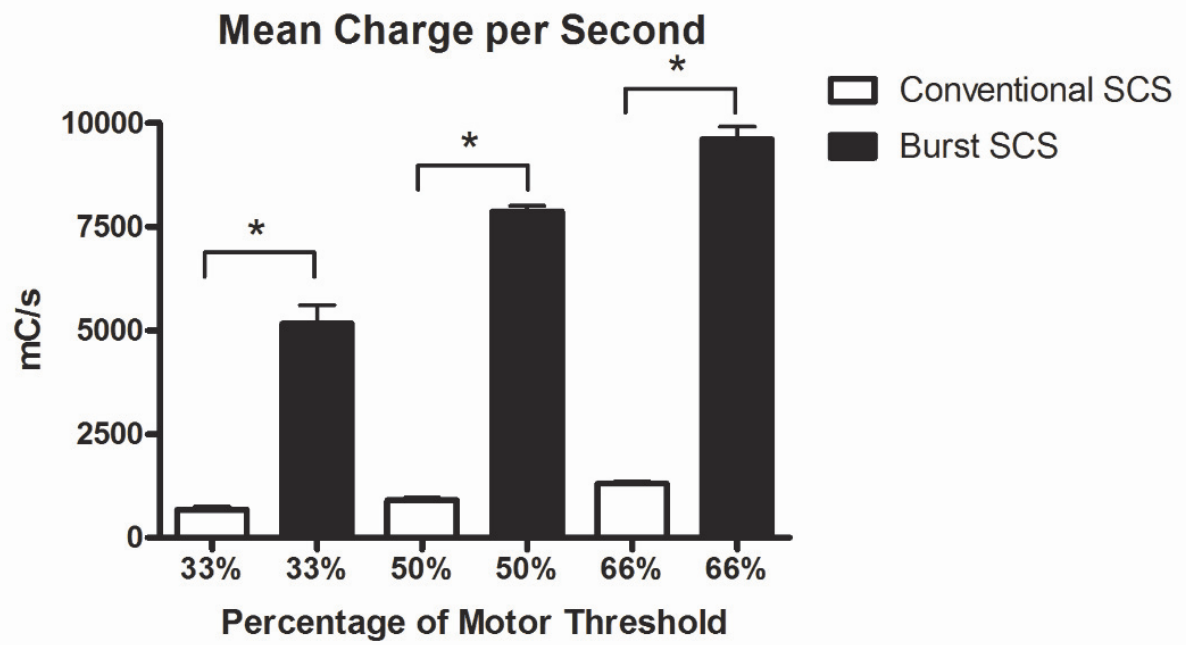

Fig. 7: The mean charge per second delivered to the spinal cord, in milliCoulomb, calculated per percentage of motor threshold for Con-SCS and Burst-SCS. $\left({ }^{*} p<0.05\right.$ for mean charge per second for Con-SCS vs. Burst-SCS).

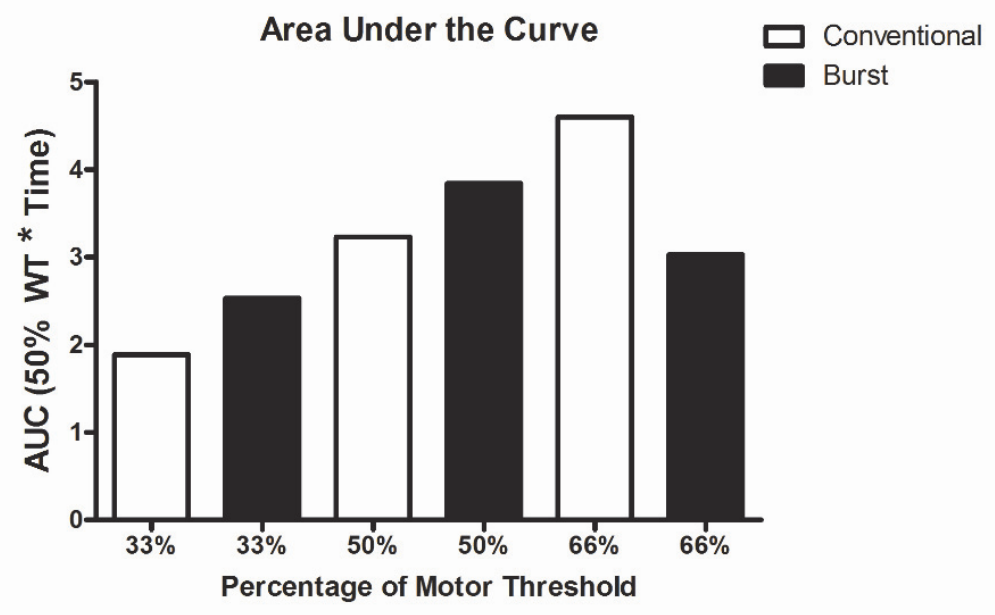

Fig.8: Area under the curve for the withdrawal thresholds per amplitude for Con-CS and Burst-SCS. 
Conventional SCS vs. Burst SCS in a Rat Model of Chronic Neuropathic Pain: Effect of Amplitude

\begin{tabular}{|c|c|c|c|c|c|c|}
\hline \multirow{2}{*}{ Time point } & \multicolumn{3}{|c|}{ Conventional SCS } & \multicolumn{3}{|c|}{ Burst SCS } \\
\hline & $33 \% \mathrm{MT}$ & $50 \% \mathrm{MT}$ & $66 \% \mathrm{MT}$ & $33 \% \mathrm{MT}$ & $50 \% \mathrm{MT}$ & $66 \%$ MT \\
\hline Baseline & $1.6 \pm 0.2 \mathrm{~g}$ & $1.8 \pm 0.3 \mathrm{~g}$ & $2.0 \pm 0.3 \mathrm{~g}$ & $1.5 \pm 0.2 \mathrm{~g}$ & $1.4 \pm 0.2 \mathrm{~g}$ & $1.2 \pm 0.2 \mathrm{~g}$ \\
\hline 15 min. SCS & $2.8 \pm 0.6 \mathrm{~g}$ & $3.2 \pm 0.7 \mathrm{~g}$ & $\begin{array}{c}8.4 \pm 1.5 \mathrm{~g} \\
* * \\
p=0.0031\end{array}$ & $1.5 \pm 0.1 \mathrm{~g}$ & $3.2 \pm 0.6 \mathrm{~g}$ & $2.4 \pm 0.2 \mathrm{~g}$ \\
\hline $30 \mathrm{~min} . \mathrm{SCS}$ & $2.4 \pm 0.7 \mathrm{~g}$ & $\begin{array}{c}7.2 \pm 1.2 \mathrm{~g} \\
* * \\
p=0.002\end{array}$ & $\begin{array}{c}11.4 \pm 0 \mathrm{~g} \\
* * \\
p=0.0013\end{array}$ & $\begin{array}{c}4.9 \pm 0.6 \mathrm{~g} \\
\quad * * \\
p=0.0056\end{array}$ & $\begin{array}{c}10.1 \pm 0.9 \mathrm{~g} \\
* * * \\
p=0.0001\end{array}$ & $\begin{array}{c}6.3 \pm 0.7 \mathrm{~g} \\
\quad * * \\
p=0.0025\end{array}$ \\
\hline $45 \mathrm{~min}$. SCS & $\begin{array}{c}2.9 \pm 0.2 \mathrm{~g} \\
\quad * \\
p=0.0125\end{array}$ & $\begin{array}{c}6.4 \pm 0.6 \mathrm{~g} \\
* * * \\
\rho=0.0006\end{array}$ & $\begin{array}{c}14.1 \pm 2.6 \mathrm{~g} \\
\quad * * \\
p=0.0024\end{array}$ & $\begin{array}{c}6.2 \pm 0.5 \mathrm{~g} \\
* * * \\
p=0.0006\end{array}$ & $\begin{array}{c}13.5 \pm 2.2 \mathrm{~g} \\
* * * \\
p=0.0008\end{array}$ & $\begin{array}{c}7.7 \pm 1.2 \mathrm{~g} \\
\quad * * \\
p=0.004\end{array}$ \\
\hline $60 \mathrm{~min}$. SCS & $\begin{array}{c}3.2 \pm 0.4 \mathrm{~g} \\
*\end{array}$ & $\begin{array}{c}6.2 \pm 0.8 \mathrm{~g} \\
\quad * * \\
p=0.0024\end{array}$ & $\begin{array}{c}11.4 \pm 2.5 \mathrm{~g} \\
\quad * * \\
p=0.0075\end{array}$ & $\begin{array}{c}5.9 \pm 0.7 \mathrm{~g} \\
\quad * * \\
p=0.0058\end{array}$ & $\begin{array}{c}14.8 \pm 2.6 \mathrm{~g} \\
\quad * * \\
p=0.0013\end{array}$ & $\begin{array}{c}8.4 \pm 1.0 \mathrm{~g} \\
* * * * \\
p=0.0001\end{array}$ \\
\hline $90 \mathrm{~min}$. SCS & $1.9 \pm 0.4 \mathrm{~g}$ & $3.4 \pm 0.6 \mathrm{~g}$ & $\begin{array}{c}6.0 \pm 0.6 \mathrm{~g} \\
\quad * * \\
p=0.0080\end{array}$ & $1.6 \pm 0 \mathrm{~g}$ & $\begin{array}{c}3.1 \pm 0.4 \mathrm{~g} \\
\quad * * \\
p=0.0074\end{array}$ & $\begin{aligned} 2.4 & \pm 0.2 \mathrm{~g} \\
& * \\
p= & 0.0121\end{aligned}$ \\
\hline
\end{tabular}

Table1: Significance values of within stimulation mode analysis. 
Chapter 2

\section{Discussion}

In the present study, it was demonstrated that Burst-SCS requires significantly more charge per second in order to achieve similar pain relief compared to Con-SCS in an experimental model of chronic neuropathic pain. Increasing amplitude with Con-SCS improved its efficacy, whereas the efficacy of Burst-SCS showed a non-monotonic relation with amplitude (Fig.8). Interestingly, Con-SCS at 66\% MT and Burst-SCS at 50\% MT were found to be equally effective in normalizing mechanical hypersensitivity. However, Burst-SCS required greater mean charge per second in order to achieve pain relief (Fig.7). Moreover, when Burst-SCS and Con-SCS were applied at a comparable mean charge per second ConSCS had a superior behavioral outcome. The amplitudes required to obtain motor thresholds were found to be significantly different, with Burst-SCS requiring significantly less amplitude in order to induce motor responses (Fig.3). The difference between Con-SCS and Burst-SCS motor thresholds, and possibly the difference in the effect of these waveforms on withdrawal thresholds, can be partially explained by their distinctive effects on affected neural structures. In particular, we note that the pulse width (PW) of the clinically-based Burst-SCS waveform $(1000 \mu \mathrm{S})$ is substantially higher than that of the clinically-based Con-SCS waveform (200 $\mu$ s pulse width). According to classical strengthduration relationships, active neurites will exhibit lower activation thresholds in response to waveforms with longer pulse widths $[29,30]$. As motor responses evoked by electrical stimulation are more likely due to neural activation than to direct muscular stimulation, the observation that MT was lower with longer PW Burst-SCS vs. shorter PW Con-SCS is expected and is consistent with findings from prior studies [17]. The importance of pulse width for the efficacy of the burst waveform was also highlighted by a recent study of Crosby and colleagues who demonstrated that burst parameters closely associated with charge delivery (pulse width, pulse number, and amplitude) were correlated with the suppression of neuronal firing of wide dynamic range neurons in the dorsal horn, whereas parameters indirectly associated with charge delivery (pulse frequency, burst frequency) did not correlate with the suppression of neuronal firing in the dorsal horn [1]. Our findings further demonstrate that Burst- and Con-SCS exhibit an essentially different behavioral pattern of pain relief, in relation to amplitude (at 66\%, 50\% and 33\% MT). For Con-SCS the amplitude is positively correlated with the behavioral outcome, as an increase in amplitude is accompanied by an increase in pain relief. Contrarily, Burst-SCS amplitude does not appear to have a positive correlation with the behavioral outcome, as $66 \% \mathrm{MT}$, the highest applied amplitude, did not produce optimal pain relief which implies a non-monotonic relation with 
amplitude. To explain this phenomenon, we first note, that the duty cycle-defined as the product of the number of stimuli with the pulse width of each stimuli divided by the total inter-burst interval-of Burst-SCS (20\%) is substantially greater than that of Con-SCS (1\%). Recently, it was hypothesized that the different outcomes of various SCS modes might be related to the active stimulation period; the balance between the ON and OFF period, i.e., Duty Cycle [31]. It is proposed that SCS could have a soothing effect on the (aberrant) dorsal horn electrical fields, potentially diminishing the (aberrant) activity of neurons that pathologically remain in a hyperactive state. [31, 32]. In line with this hypothesis, an SCS mode with a larger duty cycle like Burst-SCS will be more likely to have a substantial impact on dorsal horn electrical fields. The increased duty cycle and the high intraburst frequency of the Burst-SCS waveform act to lengthen the effective PW of the Burst-SCS waveform and preclude traditional strength-duration and charge-duration relationships from defining the relationship between single pulse parameters and neural activation thresholds [30]. Most importantly, stimulation using waveforms with longer effective pulse widths such as that used for Burst-SCS will polarize dendrites and axon terminals to a greater extent, and if stimulation is of sufficient amplitude to be supra-threshold, long effective PW waveforms may produce multiple action potentials [33]. As well, neurite polarization can alter synaptic transmission in a manner dependent on the strength of the applied stimulus [34]. With these observations in mind, we postulate that the differences in neuron polarization or activation by Burst-SCS applied at $66 \%$ MT vs. $50 \%$ may have contributed to the difference in the behavioral response of the animals to different amplitudes of Burst-SCS. However, further research is warranted to confirm 'the polarization hypothesis', as direct recordings of neuron activity were not performed in this study and it is unclear if the neural elements closest to the stimulation electrode-the dorsal columns-are actually activated or polarized by Burst-SCS [17, 35]. Along these lines, the range of amplitudes for Burst-SCS in order to achieve an optimal behavioral effect (therapeutic window) appeared to be relatively narrow given that the behavioral outcome was shown to decline rather rapidly once $50 \%$ MT (optimal Burst MT) is surpassed. This is consistent with Courtney and colleagues' finding that the therapeutic range of Burst-SCS is considerably smaller (perception amplitude $0.3 \mathrm{~mA}$, max tolerable amplitude $2.5 \mathrm{~mA}$ ) compared to Con-SCS (perception amplitude 4.2mA, max tolerable amplitude $8.9 \mathrm{~mA}$ ) [36]. However, we cannot exclude the assumption that a gradual decrease of the behavioral outcome is enclosed within the two highest amplitudes applied with Burst-SCS. It is further interesting that for Burst-SCS the analgesic effects of the two less optimal amplitudes (66\% and 33\% MT) are relatively close when compared with the less optimal amplitudes of Con-SCS (50\% and 33\% 
MT). In addition, 33\% MT Burst stimulation is able to significantly increase WTs compared to baseline WTs, whereas 33\% MT Conventional stimulation does not. Thus, Burst-SCS is effective at smaller amplitudes, yet the overall charge per second necessary to obtain a therapeutic effect is significantly higher when compared to Con-SCS. Remarkably, the large quantities of charge delivered with Burst-SCS do not result in a superior behavioral outcome, compared with Con-SCS (the maximum WT of the optimal Burst amplitude $(50 \%)$ and Conventional amplitude (66\%) did not significantly differ). This is in line with recent clinical findings from which it was concluded that stimulation with Con-SCS and Burst-SCS are equally effective in relieving neuropathic pain related to complex regional pain syndrome [37]. Furthermore, a recent systematic review concluded that the evidence for Burst-SCS in treating chronic intractable pain is considered fair and limited, AAN recommendation level $U$, with no sufficient evidence to recommend against or for BurstSCS for chronic pain reduction without paresthesia [38]. On the other hand, a 2-center comparative study on Con- versus Burst-SCS demonstrated that Burst-SCS could rescue an important amount of non-responders to conventional stimulation and could further improve pain suppression in responders to Con-SCS [14]. A possible explanation for the opposing findings was brought forward by De Ridder and Vanneste (2016), who stated that the current AAN guidelines used to assess (new) neuromodulation therapies might be an outdated methodology, and therefore the cause of negative reviews for new stimulation designs [39]. It is therefore reasonable to assume that both Con- and Burst-SCS modes are able to induce significant pain relief; despite there being significant differences in charge per second necessary for similar pain relief (Fig.7). Hence, the large pulse duration in combination with the higher pulse frequency as is required in Burst-SCS, will result in an increased energy consumption and accelerated battery depletion [38]. The significant difference in charge delivery (milliCoulomb per second) between Burst-SCS and Con-SCS seems to suggest that the amount of charge delivered to the spinal cord with SCS might not be the most important factor in pain relief. This is in line with our findings which demonstrate that when Burst-SCS and Con-SCS are applied at a comparable quantity (charge per second), Con-SCS results in a superior behavioral outcome. Recent clinical source-localized electroencephalography (EEG) data analysis suggested that burst stimulation normalizes the pain supporting/suppressing balance in contrast to tonic mode by a greater effect on the dorsal anterior cingulate cortex [35]. This is in line with previous EEG-analysis showing a more-selective modulation of the medial pain pathways by burst stimulation [40]. It was proposed that this activation normalizes a potential imbalance between ascending pain signals via the medial system and descending pain inhibitory 
activity, thereby producing superior results in comparison with conventional stimulation. This was furthermore corroborated by significant differences on the pain vigilance and awareness questionnaire following burst stimulation, which was found to improve the attention to pain and changes in pain throughout the day [40]. In this study we did not observe superior results of Burst-SCS, in comparison with Con-SCS. However, Von Frey analysis as applied in our study is a reflex-based analysis, which primarily assesses pain stimuli processed on a segmental/spinal level [41-43]. Therefore, it is likely that such a difference between Burst and Con-SCS is observed with the use of operant testing methods, addressing cognitive and motivational processing, possibly uncovering a rodent-like equivalent of pain vigilance $[41,44]$. However, it should be noted that the investigation of supraspinal pathway systems remains a research question on its own. Moreover, it is important that unambiguous parameters, such as amplitude, are assessed by means of a straightforward assessment method such as Von Frey measurement.

\section{Conclusion}

Altogether, our findings suggest that a more complex relationship defines the interplay between the parameters of SCS, the charge delivered to the spinal cord, and the therapeutic efficacy of SCS. Therefore, it is imperative that further insight is acquired into the relationship between the analgesic efficacy of Burst-SCS and its parameters (number of pulses per burst, the intraburst-interval, and the possible influence of periodicity of intraburst-interval) [45]. 


\section{References}

1. Crosby, N.D., et al., Stimulation parameters define the effectiveness of burst spinal cord stimulation in a rat model of neuropathic pain. Neuromodulation, 2015. 18(1): p. 1-8; discussion 8.

2. Kumar, K., et al., Spinal cord stimulation versus conventional medical management for neuropathic pain: a multicentre randomised controlled trial in patients with failed back surgery syndrome. Pain, 2007. 132(1-2): p. 179-88.

3. Slangen, R., et al., Spinal cord stimulation and pain relief in painful diabetic peripheral neuropathy: a prospective two-center randomized controlled trial. Diabetes Care, 2014. 37(11): p. 3016-24.

4. Geurts, J.W., et al., Spinal cord stimulation for complex regional pain syndrome type I: a prospective cohort study with long-term follow-up. Neuromodulation, 2013. 16(6): p. 523-9; discussion 529.

5. Meyerson, B.A. and B. Linderoth, Mode of action of spinal cord stimulation in neuropathic pain. J Pain Symptom Manage, 2006. 31(4 Suppl): p. S6-12.

6. Shechter, R., et al., Conventional and kilohertz-frequency spinal cord stimulation produces intensity-and frequency-dependent inhibition of mechanical hypersensitivity in a rat model of neuropathic pain. Anesthesiology, 2013. 119(2): p. 422-32.

7. Hegarty, D., Spinal cord stimulation: the clinical application of new technology. Anesthesiol Res Pract, 2012. 2012: p. 375691.

8. Yearwood, T.L., et al., Pulse width programming in spinal cord stimulation: a clinical study. Pain Physician, 2010. 13(4): p. 321-35.

9. Taylor, R.S., et al., Predictors of pain relief following spinal cord stimulation in chronic back and leg pain and failed back surgery syndrome: a systematic review and meta-regression analysis. Pain Pract, 2014. 14(6): p. 489-505.

10. Kumar, K., M. Abbas, and S. Rizvi, The use of spinal cord stimulation in pain management. Pain Manag, 2012. 2(2): p. 125-34.

11. Van Buyten, J.P., et al., High-frequency spinal cord stimulation for the treatment of chronic back pain patients: results of a prospective multicenter European clinical study. Neuromodulation, 2013. 16(1): p. 59-65; discussion 65-6.

12. Al-Kaisy, A., et al., The use of 10-kilohertz spinal cord stimulation in a cohort of patients with chronic neuropathic limb pain refractory to medical management. Neuromodulation, 2015. 18(1): p. 18-23; discussion 23.

13. Kapural, L., et al., Novel $10-\mathrm{kHz}$ High-frequency Therapy (HF10 Therapy) Is Superior to Traditional Low-frequency Spinal Cord Stimulation for the Treatment of Chronic Back and Leg Pain: The SENZA-RCT Randomized Controlled Trial. Anesthesiology, 2015. 123(4): p. 851-60.

14. De Ridder, D., et al., A 2-center comparative study on tonic versus burst spinal cord stimulation: amount of responders and amount of pain suppression. Clin J Pain, 2015. 31(5): p. 433-7.

15. de Vos, C.C., et al., Burst spinal cord stimulation evaluated in patients with failed back surgery syndrome and painful diabetic neuropathy. Neuromodulation, 2014. 17(2): p. 152-9.

16. Crosby, N.D., et al., Burst and Tonic Spinal Cord Stimulation Differentially Activate GABAergic Mechanisms to Attenuate Pain in a Rat Model of Cervical Radiculopathy. IEEE Trans Biomed Eng, 2015. 62(6): p. 1604-13.

17. Tang, R., et al., Comparison of burst and tonic spinal cord stimulation on spinal neural processing in an animal model. Neuromodulation, 2014. 17(2): p. 143-51.

18. Seltzer, Z., R. Dubner, and Y. Shir, A novel behavioral model of neuropathic pain disorders produced in rats by partial sciatic nerve injury. Pain, 1990. 43(2): p. 205-18. 
19. Truin, M., et al., Successful pain relief in non-responders to spinal cord stimulation: The combined use of ketamine and spinal cord stimulation. European Journal of Pain, 2011. 15(10).

20. Truin, M., et al., Increased efficacy of early spinal cord stimulation in an animal model of neuropathic pain. Eur J Pain, 2011. 15(2): p. 111-7.

21. Pluijms, W.A., et al., The effect of spinal cord stimulation frequency in experimental painful diabetic polyneuropathy. Eur J Pain, 2013. 17(9): p. 1338-46.

22. Chaplan, S.R., et al., Quantitative assessment of tactile allodynia in the rat paw. J Neurosci Methods, 1994. 53(1): p. 55-63.

23. Smits, H., et al., Effect of spinal cord stimulation in an animal model of neuropathic pain relates to degree of tactile "allodynia". Neuroscience, 2006. 143(2): p. 541-6.

24. Smits, H., M. van Kleef, and E.A. Joosten, Spinal cord stimulation of dorsal columns in a rat model of neuropathic pain: evidence for a segmental spinal mechanism of pain relief. Pain, 2012. 153(1): p. 177-83.

25. van Beek, M., et al., Spinal cord stimulation in experimental chronic painful diabetic polyneuropathy: Delayed effect of High-frequency stimulation. Eur J Pain, 2017. 21(5): p. 795803.

26. Cui, J.G., et al., Spinal cord stimulation attenuates augmented dorsal horn release of excitatory amino acids in mononeuropathy via a GABAergic mechanism. Pain, 1997. 73(1): p. 87-95.

27. De Ridder, D., et al., Burst spinal cord stimulation: toward paresthesia-free pain suppression. Neurosurgery, 2010. 66(5): p. 986-90.

28. Mills, C., et al., Estimating efficacy and drug ED50's using von Frey thresholds: impact of weber's law and log transformation. J Pain, 2012. 13(6): p. 519-23.

29. Geddes, L.A. and J.D. Bourland, The strength-duration curve. IEEE Trans Biomed Eng, 1985. 32(6): p. 458-9.

30. Reilly, J.P., Applied bioelectricity: from electrical stimulation to electropathology. 2012: Springer Science \& Business Media.

31. Miller, J.P., et al., Parameters of Spinal Cord Stimulation and Their Role in Electrical Charge Delivery: A Review. Neuromodulation, 2016. 19(4): p. 373-84.

32. Elbasiouny, S.M. and V.K. Mushahwar, Suppressing the excitability of spinal motoneurons by extracellularly applied electrical fields: insights from computer simulations. J Appl Physiol (1985), 2007. 103(5): p. 1824-36.

33. Bikson, M., et al., Effects of uniform extracellular DC electric fields on excitability in rat hippocampal slices in vitro. J Physiol, 2004. 557(Pt 1): p. 175-90.

34. Rahman, A., et al., Cellular effects of acute direct current stimulation: somatic and synaptic terminal effects. J Physiol, 2013. 591(10): p. 2563-78.

35. De Ridder, D. and S. Vanneste, Burst and Tonic Spinal Cord Stimulation: Different and Common Brain Mechanisms. Neuromodulation, 2016. 19(1): p. 47-59.

36. Courtney, P., et al., Improved Pain Relief With Burst Spinal Cord Stimulation for Two Weeks in Patients Using Tonic Stimulation: Results From a Small Clinical Study. Neuromodulation, 2015. 18(5): p. 361-6.

37. Kriek, N., et al., Preferred frequencies and waveforms for spinal cord stimulation in patients with complex regional pain syndrome: A multicentre, double-blind, randomized and placebocontrolled crossover trial. Eur J Pain, 2017. 21(3): p. 507-519.

38. Hou, S., K. Kemp, and M. Grabois, A Systematic Evaluation of Burst Spinal Cord Stimulation for Chronic Back and Limb Pain. Neuromodulation, 2016. 19(4): p. 398-405.

39. De Ridder, D. and S. Vanneste, Response: A Systematic Evaluation of Burst Spinal Cord Stimulation for Chronic Back and Limb Pain. Neuromodulation, 2016. 19(7): p. 785-786.

40. De Ridder, D., et al., Burst spinal cord stimulation for limb and back pain. World Neurosurg, 2013. 80(5): p. 642-649 e1. 
Chapter 2

41. Mogil, J.S., Animal models of pain: progress and challenges. Nat Rev Neurosci, 2009. 10(4): $p$. 283-94.

42. Woolf, C.J., Long term alterations in the excitability of the flexion reflex produced by peripheral tissue injury in the chronic decerebrate rat. Pain, 1984. 18(4): p. 325-43.

43. Matthies, B.K. and K.B. Franklin, Formalin pain is expressed in decerebrate rats but not attenuated by morphine. Pain, 1992. 51(2): p. 199-206.

44. Harte, S.E., et al., Mechanical Conflict System: A Novel Operant Method for the Assessment of Nociceptive Behavior. PLoS One, 2016. 11(2): p. e0150164.

45. Huh, Y. and J. Cho, Discrete pattern of burst stimulation in the ventrobasal thalamus for antinociception. PLoS One, 2013. 8(6): p. e67655. 


\section{CHAPTER 3}

\section{Burst spinal cord stimulation in peripherally injured chronic neuropathic rats: a delayed effect.}

Koen P.V. Meuwissen, Jianwen Wendy Gu, Tianhe C. Zhang, Elbert A. J. Joosten.

Pain Practice, Volume 18, Issue 8, 2018 988-996.

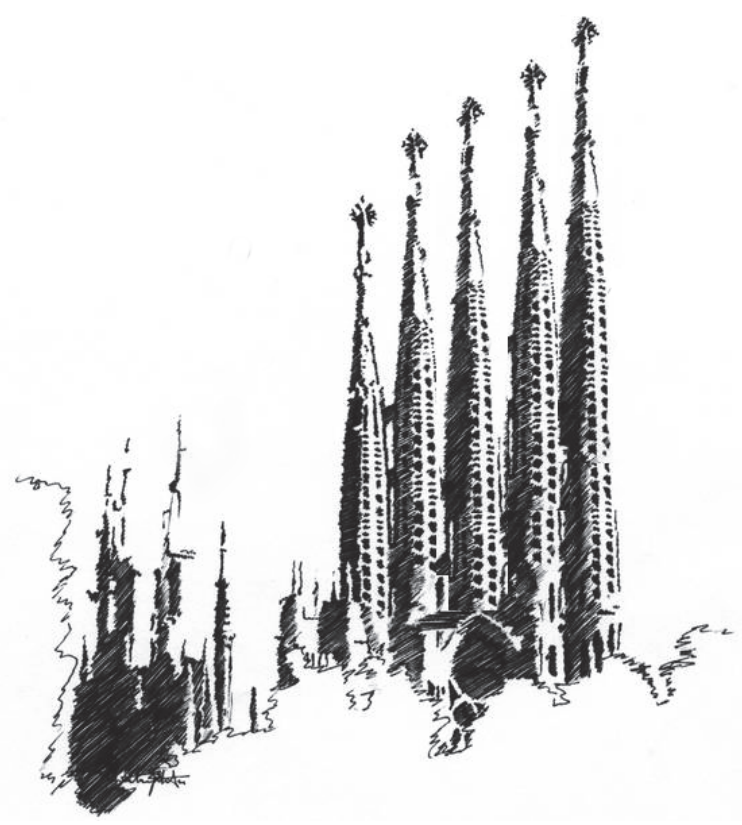




\section{Abstract}

OBJECTIVE: Two well-known spinal cord stimulation (SCS) paradigms, Conventional (Con) and Burst-SCS, are hypothesized to exert their anti-nociceptive effects through different stimulation-induced mechanisms. We studied the course of the behavioral anti-nociceptive effect during 60 minutes of SCS and 30 minutes post SCS, in a rat model of chronic neuropathic pain.

MATERIAL AND METHODS: Animals received a unilateral partial sciatic nerve ligation, after which they were implanted with quadripolar electrodes into the epidural space at vertebral level T13 ( $n=43$ rats). While receiving either Con-SCS or biphasic Burst-SCS, pain behavior of the rats was assessed by means of Paw Withdrawal Thresholds (WT) in response to the application of Von Frey monofilaments.

RESULTS: After 15 minutes of Con-SCS ( $n=21$ ), WT's significantly differed from baseline ( $p=$ $0.04)$, whereas WT's of the Burst-SCS ( $n=22)$ group did not. After 30 minutes of SCS, WT's of the Con-SCS and Burst SCS group reached similar levels, both significantly different from baseline, indicating a comparable anti-nociceptive effect for these SCS-paradigms. Yet, the WT's of the Burst-SCS group were still significantly increased compared with baseline at 30 minutes post stimulation, whereas the WT's of the Con-SCS group did not.

CONCLUSIONS: To conclude, biphasic Burst-SCS results in a delayed anti-nociceptive effect after onset of the stimulation, as compared with Con-SCS, in a chronic neuropathic pain model. Furthermore, biphasic Burst-SCS seems to exhibit a delayed wash-out of analgesia after stimulation is turned off. 


\section{Introduction}

Over the last four decades, spinal cord stimulation (SCS) has become a safe and effective therapeutic tool for the treatment of various chronic pain conditions [1-3]. Conventional (Con), or tonic, SCS has proven to be effective for the management of various selected chronic neuropathic pain conditions, often refractory to pharmacological tools, like Failed Back Surgery Syndrome and Complex Regional Pain Syndrome [2-5]. Con-SCS administers continuous pulses of electrical stimulation to the dorsal columns of the spinal cord with frequencies within the range of $40-60 \mathrm{~Hz}[6,7]$. The SCS effect emerges from electrodes implanted in the epidural space between the spinal cord and the vertebral column. Despite the positive outcome, the reported success rate of Con-SCS in clinical studies has improved little over the last decade and therefore remains suboptimal, with roughly $50 \%$ pain relief for $50 \%$ of the patients $[4,8]$. In an effort to improve the efficacy of SCS several alternative stimulation paradigms have been developed. One such example is Burst-SCS; a paradigm which uses periodic bursts of pulses rather than the continuous pulses as used in Con-SCS $[9,10]$. Clinical studies, including placebo controlled studies, have suggested that Burst-SCS decreases pain intensity to a greater degree than Con-SCS $[9,11]$. However, recent evidence shows that pain relief with Burst-SCS is not necessarily better than Con-SCS, and different patients might have a preference for different SCS-modalities $[12,13]$. It thus seems that Burst-SCS remains to be optimized, and to that end fundamental research is required. Preclinical studies have already provided insights into the working mechanisms of Burst-SCS by investigating its electrophysiological effects in the spinal cord [14] and describing the associated neurochemical mechanisms [15]. Yet, on a behavioral level the literature remains rather restricted, with one study demonstrating that Burst-SCS reduces hyperalgesia and restores physical activity levels more so than Con-SCS, and one study which assesses the effect of various amplitudes on mechanical hypersensitivity for Burstand Con-SCS $[16,17]$. Therefore, a better understanding of the behavioral anti-nociceptive effect of Burst-SCS as compared to Con-SCS in an experimental neuropathic pain model is needed. This is important in view of further optimization of the Burst-SCS paradigm and might have serious implications for its clinical use in the treatment of neuropathic pain. 


\section{Materials and Methods}

\section{Ethics Statement}

All experiments were performed in accordance with the European Directive for the Protection of Vertebrate Animals Used for Experimental and Other Scientific Purposes (86/609/EU). The protocol was approved by the Animal Research Committee of the Maastricht University Medical Centre (DEC-protocol 2014-086).

\section{Animals}

All experiments were performed using male Sprague Dawley rats $(n=52$, which were youngadult ( 5 weeks of age) at the start of the experiment (150-200 g). Animals were housed in groups of 2, in filter-top polycarbonate cages in a climate-controlled vivarium maintained under controlled temperature $\left(21 \pm 1^{\circ} \mathrm{C}\right)$, relative humidity $(55 \pm 15 \%)$ and artificial lighting (12:12 light/dark cycle) with distilled water and rodent food available ad libitum. The vivarium was equipped with a mobile radio, continuously producing background music at 45 decibel, in order to desensitize the animals for translocation and experimenter related noise. All procedures were conducted between 09:00 and 16:00 hours.

\section{Partial Sciatic Nerve Ligation (PSNL)}

A unilateral ligation of the left sciatic nerve was performed as described by Seltzer et al. 1990 [18], and previously applied in our laboratory $[19,20]$. In short, animals were anesthetized with 3-5\% isoflurane (Abbott Laboratories Ltd., Kent, U.K.) and air enriched with $100 \%$ oxygen at a constant flow rate of $250 \mathrm{ml} / \mathrm{min}$. Body temperature was maintained at $37.5^{\circ} \mathrm{C}$ using an automatic heating pad. The sciatic nerve from the left hind paw was exposed by blunt dissection. Upon discovery the nerve was carefully freed from surrounding connective tissue and partially (approximately $1 / 3$ ) ligated by an $8 / 0$ non-absorbable silk suture with a reverse cutting micro needle. Post ligation, the wound was closed with a $4 / 0$ silk suture, and the development of tactile hypersensitivity (mechanical allodynia) was monitored over the following 14 days. The presence of mechanical hypersensitivity was confirmed at 14 days post- 100 surgery, only if the log $(50 \%$ withdrawal threshold (WT)) was decreased by 0.2 units compared to 101 baseline (day 0) [21]. 


\section{Assessment of mechanical hypersensitivity (von Frey assay)}

Mechanical hypersensitivity was assessed using von Frey filaments, as described in Van Beek et al. [22]. Von Frey testing was always conducted in the same room, which was isolated from all external sound and light sources, maintained under controlled temperature $\left(21 \pm 1^{\circ} \mathrm{C}\right)$ and relative humidity $(55 \pm 15 \%)$. The test-room was equipped with a mobile radio, continuously producing background music at 45 decibels. WT to von Frey filaments was assessed using the 'up-down method' [23] whereby von Frey filaments with approximately linearly incrementing stiffness on a logarithmic scale were applied to the plantar surface of the hind paws of the rats for 5 seconds. For statistical analysis the $50 \%$ WTs were logarithmically transformed to yield a linear scale.

\section{Implantation of spinal cord stimulation device}

The implantation of the SCS device was performed according to the standard protocol used in our institution $[20,24,25]$, which was based on the method originally developed at the Karolinska Institute [26]. In short, the spinal cord was exposed by a midline, lumbar incision, after topical administration of $0.5 \%$ lidocaine (Xylocain, $50 \mathrm{mg} / \mathrm{g}$, AstraZeneca) followed by laminectomy at level T13. During the full procedure, the dura was kept intact. A custommade cylindrical 4-contact lead $(0.72 \mathrm{~mm}$ diameter; Boston Scientific Neuromodulation, Valencia, CA, USA) was introduced into the epidural space. The electrode was put caudally below the adjacent 1 or 2 lamina. Electrode configuration was set at alternating cathode and anode settings (rostral to caudal: + - + -). Subsequently, the electrode was secured to a spinous process with tissue adhesive (Histoacryl ${ }^{\circledR}$, B Braun Medical BV, Oss, the Netherlands) to prevent electrode migration. The electrode wires were tunneled subcutaneously to the animal's neck and the stimulator-connectors were attached to the skin. After implantation of the electrodes, the rats were given 2 days for recovery prior to the initiation of SCS.

\section{Spinal cord stimulation}

For stimulation of the dorsal columns an A-M systems stimulator (MultiStim: Programmable 8-Channel Stimulator (Model 3800) $220 \mathrm{~V} / 50 \mathrm{~Hz}$ ) fitted with a stimulus Isolator (Model 3820 for A-M Systems MultiStim) was used. For all experiments, the stimulator was set to deliver constant current biphasic stimulation for both the Conventional and Burst modes. Con-SCS Motor Threshold (MT) was determined at the following settings: pulse width of $200 \mu \mathrm{S}$ administered at a frequency of $2 \mathrm{~Hz}$. Burst-SCS MT was determined at the following settings: pulse width of $1000 \mu \mathrm{S}$, five pulses (449 $\mathrm{Hz}$ intra-burst frequency) administered at an 
interburstfrequency of $2 \mathrm{~Hz}$. The amplitude was gradually increased until symmetrical contractions of the lower trunk and/or hind limbs were visually observed or perceived by hand. Then, either a Con-SCS paradigm (frequency $50 \mathrm{~Hz}$, pulse width $200 \mu \mathrm{S}$ ) [20], or a biphasic Burst-SCS paradigm (interburst-frequency $40 \mathrm{~Hz}$, pulse width $1000 \mu \mathrm{S}, 5$ spikes at $449 \mathrm{~Hz}$ intra-burst frequency), was applied at 66\% MT for 60 minutes [10, 17] (Fig.1). In contrast to literature of de Ridder et al., who applies monophasic BurstDR-stimulation, we applied biphasic Burst-stimulation [9-11, 27]. In all experiments, the WT to von Frey filaments was assessed before the start of SCS treatment, at 15', 30', 60' min. after stimulation was turned on, and at $30 \mathrm{~min}$. after stimulation was turned off ( $90 \mathrm{~min}$, after the start of SCS). The investigator was blinded to the stimulation condition during behavioral testing.

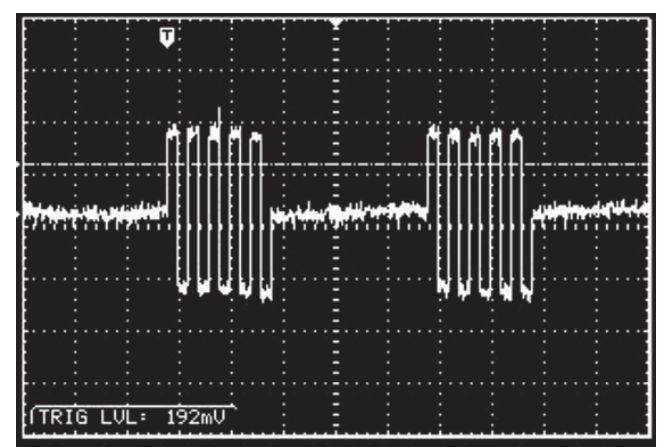

Fig.1: Constant current burst mode $(\mu \mathrm{A}): 1-\mathrm{ms}$ biphasic spikes with a 1.1-ms spike interval $(449-\mathrm{Hz}$ spike mode) at $40 \mathrm{~Hz}(40-\mathrm{Hz}$ burst mode). Stimulation delivered by the A-M systems stimulator (MultiStim: Programmable 8-Channel Stimulator (Model 3800). (C) 2018 Wiley \& Sons, Inc. all rights reserved.

\section{Timeline of experiments}

After acclimatization to the vivarium, 52 animals underwent a partial sciatic nerve ligation (PSNL) (as described in section 2.3). During the subsequent 14 day observation period, animals received Von Frey measurements in order to assess the development of tactile hypersensitivity (see section 2.3 and Fig.2). Animals that had not developed tactile hypersensitivity at day 14 post ligation (DPL14) were excluded from the study and did not receive implantation of the SCS electrode. The 46 animals which had successfully developed tactile hypersensitivity received implantation of an SCS-electrode at DPL14, as described in section 2.5. Animals for which the SCS-electrode was successfully implanted $(n=45)$ were given two days to recover from the surgery before the Motor-Threshold (MT) was assessed 
at DPL17. Prior to MT-assessment, animals were connected to the stimulator as if to receive stimulation. Animals with a MT deviating more than two standard deviations from the mean and/or animals with a MT which deviated more than $500 \mu \mathrm{A}$ over the MT-assessment days were excluded from the study. Animals which did not show a robust MT, were excluded from the study and did not receive SCS (two animals did not show a robust MT). The remaining animals that showed a robust MT (n=43), at DPL17, were randomly divided over the Con-SCS or Burst-SCS group. Mechanical hypersensitivity was assessed, by Von Frey WT assessment, at 15, 30, 40 and 60 stimulation of sham-stimulation (amplitude was set at 0), and 90 minutes post sham-stimulation. At DPL18 animals received SCS for 60 minutes. Mechanical hypersensitivity was assessed based on WT to von Frey filaments, at 15, 30, 40 and 60 of stimulation, and 90 minutes post stimulation.

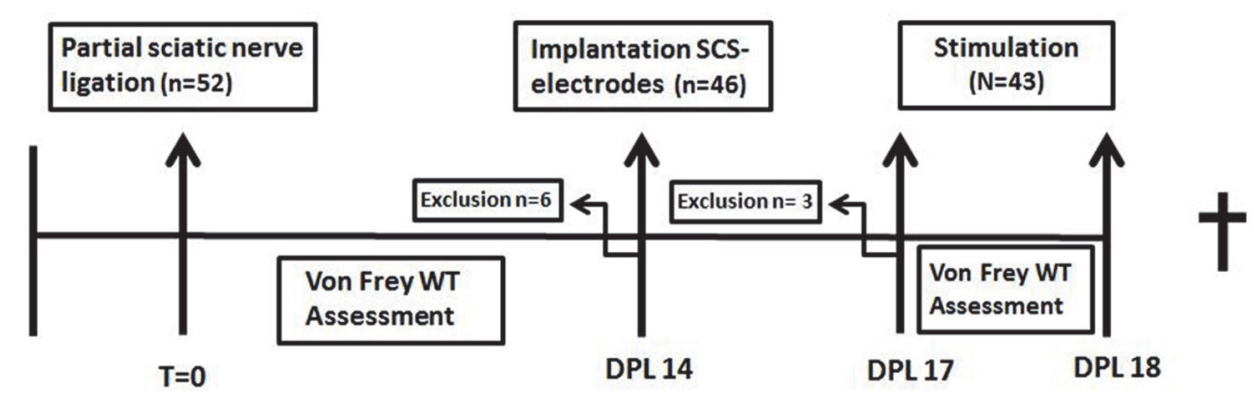

Fig. 2: Timeline of experiments

\section{Data Analysis}

The WT's to von Frey filaments are presented as mean \pm standard error of the mean (SEM). For statistical analysis von Frey data were logarithmically transformed to obtain a linear scale and to account for Weber's Law [28]. For statistical analysis of differences in the withdrawal thresholds over time within groups, the non-parametric Friedman test was used followed by followed by Dunn's post hoc test. For the analysis of differences in the withdrawal thresholds between groups, ipsilateral and contralateral withdrawal thresholds, the nonparametric Mann-Whitney-U-test was used. All statistical analyses were performed with $\alpha=0.05$ using IBM SPSS statistics 23 . 


\section{Results}

\section{Development of tactile hypersensitivity}

Pre-nerve injury, WT's of the ipsilateral hind paws were comparable with the WT's of the contralateral hind paws (ipsilateral $12.4 \pm 0.8 \mathrm{~g}$ vs. contralateral $12.3 \pm 1 \mathrm{~g}$ ) in all animals. Out of a total of 52 animals that received a unilateral partial ligation of the sciatic nerve, 6 animals did not develop tactile hypersensitivity, and were excluded from the study. The remaining 46 animals qualified as hypersensitive to mechanical stimulation by von Frey filaments ( $p<0.01$; ipsilateral average WT's: $12.4 \pm 0.8 \mathrm{~g}$ (pre-lesion) to $1.2 \pm 0.4 \mathrm{~g}$ (DPL14)) (see Methods section 2.3 and Fig.3) and were selected for the implantation of the SCS electrodes study. Of the 46 animals which received an SCS-electrode, 43 were found eligible for the SCS-experiments (Fig. 2). The ipsilateral ligation had no significant effect on the withdrawal thresholds of the contralateral WT's $(p=0.42$; pre-surgery $12.3 \pm 1 \mathrm{~g}(\mathrm{n}=43)$ vs. post-surgery $13.1 \pm 0.8 \mathrm{~g}$ contralateral $\left.W T^{\prime} s(n=43)\right)$.

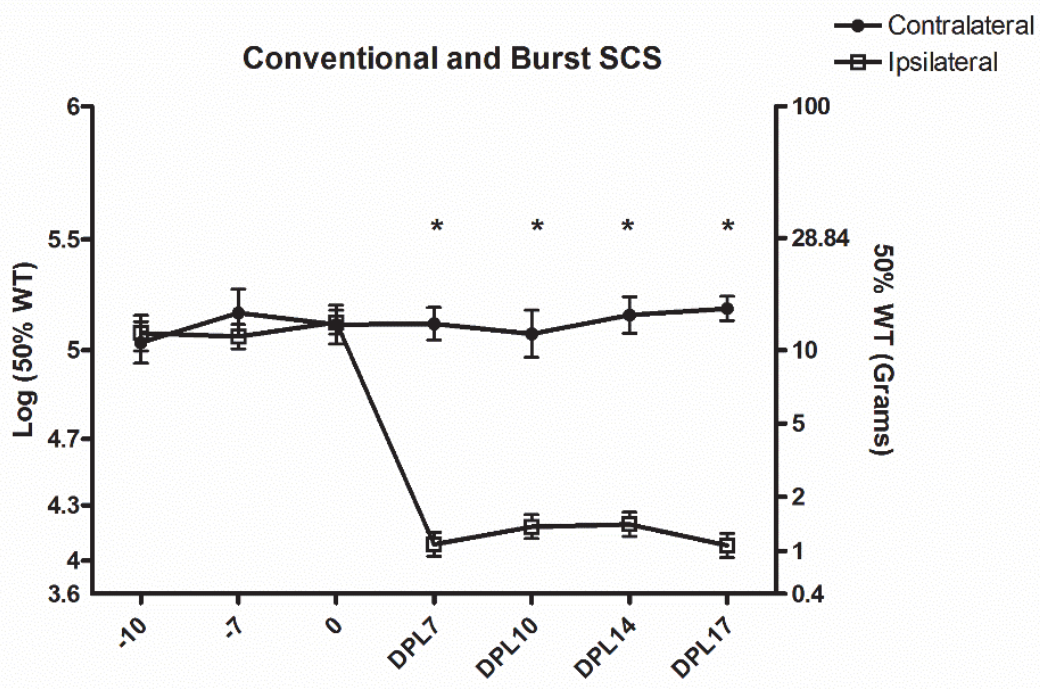

Fig.3: Development of tactile hypersensitivity for the SCS-selected animals, based on paw withdrawal threshold to von Frey hair stimulation after unilateral sciatic nerve ligation in adult rat $(n=43)$. ${ }^{*} p<$ 0.05 for ipsilateral versus contralateral WT's. 


\section{Spinal Cord Stimulation}

Animals received 60 minutes of SCS at $66 \%$ MT ( $n=43$; see section 2.6 of Methods and Fig.2). The average motor thresholds for Con-CS $(135 \pm 4 \mu \mathrm{A})$ and Burst-SCS $(57 \pm 3.5 \mu \mathrm{A})$ were found to be statistically different $(p<0.01)$ (Fig.4). WTs to von Frey filaments was assessed before the start of SCS treatment, at 15', 30', 60' min. after stimulation was turned on, and at $30 \mathrm{~min}$. after stimulation was turned off (90 min, after the start of SCS). No significant differences were observed in ipsilateral paw withdrawal thresholds (WT's) at baseline, preSCS, between groups ( $p=0.62$; Con-SCS $1.2 \pm 0.4 \mathrm{~g}[\mathrm{n}=21]$ vs. Burst-SCS $1.5 \pm 0.7 \mathrm{~g}[\mathrm{n}=22]$ ). During SCS WT's of both the Con-SCS group $\left(X^{2}=89.7 ; p<0.001\right)$ and Burst-SCS group $\left(X^{2}=90.5 ; p<0.001\right)$ significantly differed over time, while the Sham-SCS did not $\left(X^{2}=15.9 ; p\right.$ $=0.7)$. After 15 mins of SCS WT's of the Con-SCS group were significantly increased, compared with baseline WT's ( $p=0.03 ; 1.2 \pm 0.4 \mathrm{~g}$ vs $8.6 \pm 1.3 \mathrm{~g}$ ). Meanwhile, after 15 mins of SCS, WT's of the Burst-SCS group did not significantly differ from baseline WT's ( $p=0.16$; $1.5 \pm 0.7 \mathrm{~g}$ vs $3.5 \pm 1.7 \mathrm{~g}$ ). Furthermore, after 15 mins of stimulation WT's of the Con-SCS group and Burst-SCS group significantly differed ( $p=0.04$; Con-SCS: $8.6 \pm 1.3 \mathrm{~g}$ vs Burst SCS: $3.5 \pm 1.7 \mathrm{~g}$ ) (Fig.5+6). After 30 mins of stimulation, 45 mins of stimulation, and 60 mins of stimulation WT's of both the Burst-SCS group ( $p=0.02, p=0.03$ and $p=0.02$ respectively) and Con-SCS group ( $p=0.03, p=0.03$ and $p=0.03$ respectively) significantly differed from baseline WT's. Also, WT's of the Burst-SCS group and Con-SCS group did not significantly differ at 30 mins of stimulation ( $p=0.63), 45$ mins of stimulation $(p=0.75)$, and 60 mins of stimulation ( $p=0.47$ ) (Fig.5). However, at 30 mins post stimulation WT's of the Burst-SCS group were still significantly higher as compared with baseline $(p=0.04 ; 1.5 \pm 0.7 \mathrm{~g}$ vs $5.5 \pm$ $2.6 \mathrm{~g}$ ), whereas WT's of the Con-SCS group were not ( $p=0.5 ; 1.2 \pm 0.4 \mathrm{~g}$ vs $1.9 \pm 1.1 \mathrm{~g}$ ). Furthermore, at 30 mins post stimulation we observed a strong trend for the difference between WT's of the Con-SCS group and Burst-SCS group ( $p=0.06$; Con-SCS: $1.9 \pm 1.1 \mathrm{~g}$ vs Burst SCS: $5.5 \pm 2.6 \mathrm{~g}$ ) (Fig.5+6). 


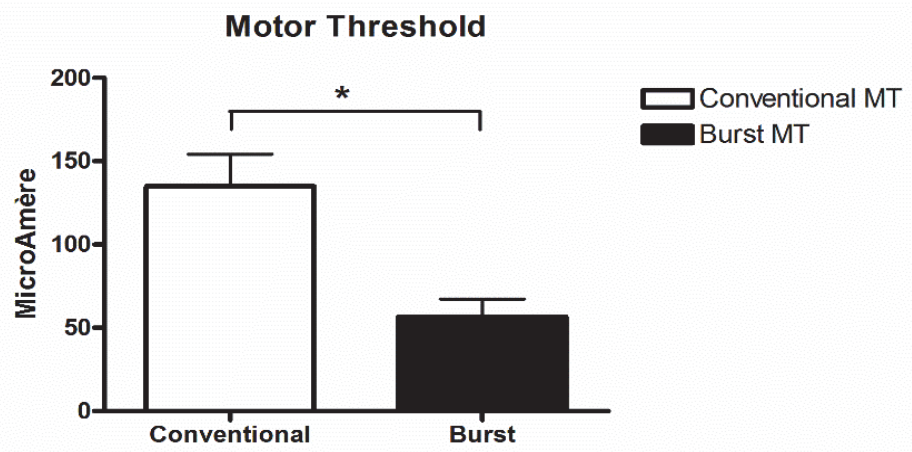

Fig.4: Motor threshold (MT) assessed by means of Conventional and Burst stimulation. Con-SCS MT was determined at the following settings: pulse width of $200 \mu \mathrm{S}$ administered at a frequency of $2 \mathrm{~Hz}$. Burst-SCS MT was determined at the following settings: pulse width of $1000 \mu \mathrm{S}$, five pulses $(500 \mathrm{~Hz}$ intraburst-frequency) administered at an interburst-frequency of $2 \mathrm{~Hz}$. (* $p<0.05$ for Con-MTs versus Burst-MTs).

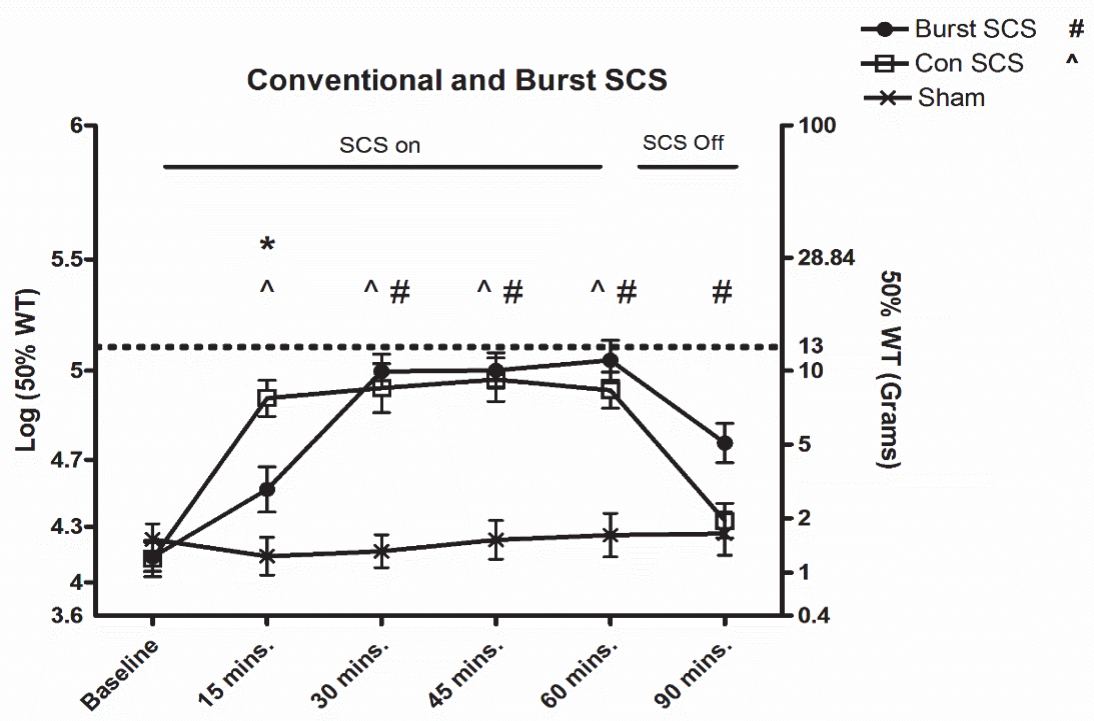

Fig.5: The effect of Con-SCS ( $n=21)$ and Burst-SCS $(n=22)$ on the paw withdrawal thresholds (WT's) based on sensitivity to von Frey filaments. WT's were assessed at 15, 30, 45, and 60 mins of stimulation and 30 mins. post stimulation. The dotted line represents the average WT baseline prior to sciatic nerve ligation (\# $p<0.05$ for Burst-SCS vs. baseline; ${ }^{\wedge} p<0.05$ for Con-SCS vs. baseline; ${ }^{*} p<0.05$ for Burst-SCS vs Con-SCS). 


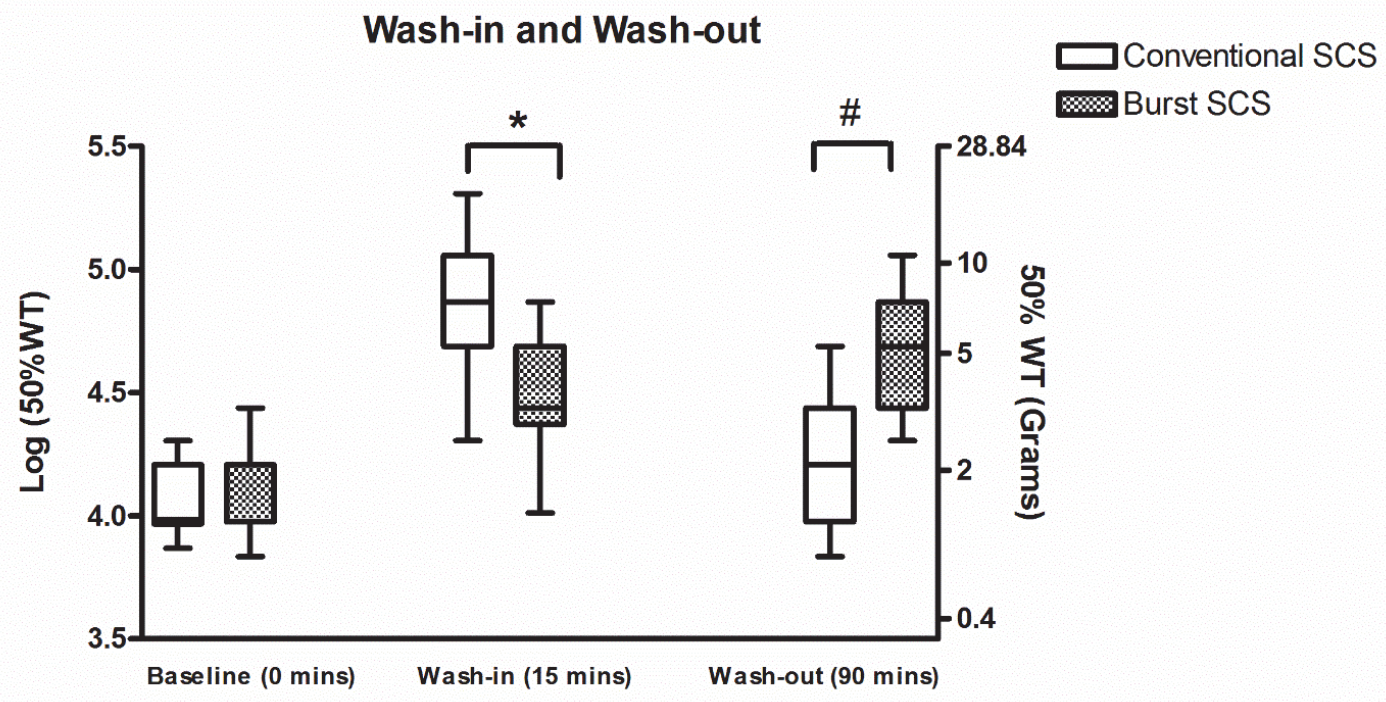

Fig.6 Boxplot of the WT's of Con-SCS and Burst-SCS for the wash-in period (15 minutes of SCS) and wash-out period (30 minutes post-SCS). (* $p<0.05$ for Burst-SCS vs Con-SCS, \# $p<0.07$ for Burst-SCS vs. Con-SCS)

\section{Discussion}

The aim of this study was to evaluate the behavioral anti-nociceptive effect of biphasic Burst-SCS, as compared with Con-SCS, on mechanical hypersensitivity in an experimental chronic neuropathic pain model. We report a delayed anti-nociceptive effect of Burst-SCS after onset of the stimulation, as compared with Con-SCS. Furthermore, Burst-SCS seems to exhibit a delayed wash-out of analgesia after stimulation is turned off. However, no difference in maximum anti-nociceptive effect of Burst-SCS, as compared with Con-SCS, was noted in this model of chronic neuropathic pain. The amplitudes required to obtain motor thresholds were found to be significantly different, with Burst-SCS requiring significantly less amplitude in order to induce motor responses (Fig.4). This is in line with a recently published study of Meuwissen et al. (2018), where a similar difference between Burst-MT and Conventional-MT was reported [17]. Since the introduction of Burst-SCS for the treatment of chronic neuropathic pain in 2010, several mechanistic studies have been performed in order to define the working mechanisms and improve its effectiveness [14, 15]. On the other hand, the behavioral anti-nociceptive effect of Burst-SCS in experimental studies has received little attention. A recent behavioral study demonstrated that Biphasic 
Burst-SCS requires significantly more mean charge per second in order to achieve similar pain relief, as compared with Con-SCS, in an experimental model of chronic neuropathic pain [17]. In this study the researchers observed that with increasing amplitude Con-SCS improved its efficacy, whereas the efficacy of Burst-SCS showed a non-monotonic relation with amplitude. Furthermore, a non-significant delayed effect of Burst-SCS was observed. However, due to the small number of animals this effect was not substantiated. Another behavioral study demonstrated that Burst-SCS reduces hyperalgesia and restores physical activity levels more than tonic stimulation at standard frequencies in a neuropathic animal model based on spared nerve lesions[16]. Animals showed an increase in paw withdrawal threshold and physical activity levels during stimulation with Burst-SCS, which was found to be more effective than sham and tonic SCS. Thus, in contrast to our findings they reported a superior anti-nociceptive effect of Burst-SCS, as compared with Con-SCS. However, it is important to note that neuropathic pain was induced using the spared nerve injury model, which is known to be more severe as compared with the partial sciatic nerve ligation model applied in our study $[29,30]$. This seems to support the idea that specific waveforms may be selectively beneficial for specific conditions $[9,31]$. This is reflected in the clinical setting where Burst-SCS was demonstrated to be more effective in providing additional pain reduction for specific conditions [13]. Secondly, the pulse width of Con-SCS applied in our study was less than half the pulse width used by Gong and colleagues ( $200 \mu \mathrm{sec} v \mathrm{~s} 500 \mu \mathrm{sec}$ respectively) [16]. As Con-SCS is generally applied with a pulse width of $\pm 200 \mu \mathrm{sec}$, it is conceivable that the animals in their study experienced more side-effects during SCS (paresthesia), prohibiting an optimal anti-nociceptive effect of Con-SCS [32]. Remarkably, Gong and colleagues did also not report a delayed effect of Burst-SCS, as compared with Con-SCS [16]. However, it should be noted that, during SCS, paw withdrawal thresholds (WT's) were not assessed in a repeated fashion. Therefore, it is plausible that a potential delayed effect of Burst-SCS remained undetected. Interestingly, a delayed behavioral effect of High Frequency SCS (HF-SCS; $500 \mathrm{~Hz}$ ), as compared with standard tonic SCS $(50 \mathrm{~Hz})$, has been reported in chronic painful diabetic polyneuropathy animals [22]. Interestingly, in a recent review it was hypothesized that $10 \mathrm{kHz}$ and Burst-SCS might both modulate the medial pain pathway, and could be fundamentally similar neurostimulation designs [33]. The similarity regarding (delayed) effect onset observed in our study and the study by Van Beek et al. does seem to suggest that Burst-SCS and High-Frequency-SCS rely on similar neuronal pathways and it would be interesting to further investigate the mechanisms involved with these SCS-paradigms. Being as the latter findings are related to diabetic polyneuropathy it was hypothesized that the HF-SCS increased vasodilation via antidromic 
stimulation of unmyelinated C-fibers, treating the pain etiotropically [34]. In contrast, ConSCS is considered a symptomatic treatment, which covers the affected painful area with paresthesia. It thus seems that SCS delivered with specific parameter settings does affect different physiological processes. Indeed, computer modelling has indicated that SCS frequency, at least partly, determines the firing of Wide Dynamic Range (WDR) neurons projecting to the brain [35]. It was demonstrated that SCS with frequencies above $100 \mathrm{~Hz}$ do not result in inhibition of WDR signaling; this strongly suggests that higher SCS frequencies induce pain relief via alternative mechanisms. Although it should be taken into account that the frequencies tested were most likely applied at supra-perception amplitudes, in contrast to the sub-perception amplitudes at which Burst-SCS was applied in our study. Along these lines, it has been suggested that Burst-SCS and Con-SCS mediate their anti-nociceptive effect through different underlying mechanisms [9]. This is corroborated by preclinical electrophysiological studies, indicating that Burst-SCS might not rely on the activation of $G_{A B A}$ receptors and thus not on presynaptic modulation of GABA release for the inhibition of nociceptive $C$ - and $A_{d}$-fiber, whereas Con-SCS does $[15,36]$. Furthermore, the effect of orthodromic stimulation of the $A \beta$-fibers in the dorsal columns is suggested to differ between Burst- and Con-SCS: Burst-SCS was found not to increase spontaneous activity of gracile nucleus neurons in the brainstem, whereas Con-SCS did [37]. These findings suggest that the anti-nociceptive effect of Burst-SCS and Con-SCS might be established via modulation of fundamentally different pain pathways. This is in line with clinical studies, which, based on FMRI and EEG analyses, demonstrate that the antinociceptive effect mediated via Burst-SCS includes the modulation of the medial pain pathways, whereas Con-SCS does not $[9,38]$. The medial pain pathway is associated with the supraspinally processed affective and attentional components of pain. Following this line of reasoning, it might be possible that the stimulation of specific pathways via different SCS-paradigms requires a different timeframe in order to fully establish its anti-nociceptive properties. Thus, this might underlie the onset timing difference between the behavioral effects of Burst- and Con-SCS as observed in our study. A difference in effect-onset for BurstSCS and Con-SCS is furthermore in line with unpublished clinical anecdotes. Furthermore, it is known that the medial pain pathway is associated with attention (for pain), which has led to the hypothesis that Burst-stimulation might exert its main effect by an attentionmodulating effect [9]. Building on this hypothesis it is conceivable that such an attentionmodulation effect, mediated by brain structures associated with the limbic system, reverberates after cessation of stimulation, as compared to the processing of sensorydiscriminative components of pain as seen with Con-SCS. Therefore, one could hypothesize 
that the attention-modulating effect of Burst-SCS might echo on after the stimulation is terminated, which is reflected by a delayed wash-out effect, as compared with Con-SCS. Yet, future experiments should be performed in order to shed more light on the differences in pathway activation associated with different waveforms. However, it should be noted that the biphasic Burst-waveform, as applied in our study, has not yet been demonstrated to modulate the medial pain pathway. Future studies should elucidate whether there is a difference in pathway activation for monophasic BurstDR-SCS and biphasic Burst-SCS. In this study we did not observe superior results of Burst-SCS, in comparison with Con-SCS. Yet, Von Frey analysis as applied in our study is known to be a reflex-based assessment method which primarily assesses pain stimuli processed on a segmental/spinal level [39-41]. Future studies assessing the effect of Burst- versus Con-SCS by means of a non-reflex based operant testing method, in which supraspinal aspects of pain are assessed, could potentially uncover a superior effect for Burst-SCS. An innovative example is the Mechanical Conflict-Avoidance System (MCAS), an operant testing method for preclinical pain assessment, which uses painmotivated behavior to capture cognitive and motivational aspects of pain in rodents [42]. It should be noted that the investigation of supraspinal elements of pain remains a research question on its own. Hence, it is important that unambiguous stimulation aspects, such as a wash-in and/or wash-out effect, are assessed by means of a straightforward assessment method such as Von Frey measurement.

\section{Limitations}

Only one behavioral assessment method was used. As Burst-SCS is hypothesized to recruit the medial-spinothalamic tract future studies should include the analysis of emotionalaffective aspects of pain in animal studies. An innovative example is the Mechanical Conflict-Avoidance System (MCAS), an operant testing method for preclinical pain assessment, which uses pain-motivated behavior to capture cognitive and motivational aspects of pain in rodents [42]. 


\section{Conclusion}

To conclude, biphasic Burst-SCS results in a delayed anti-nociceptive effect after onset of the stimulation, as compared with Con-SCS. Furthermore, Burst-SCS seems to exhibit a delayed wash-out of analgesia after stimulation is turned off. Yet, the maximum antinociceptive effect based on paw withdrawal responses did not differ between Burst- and Con-SCS. The maximum anti-nociceptive effect based on paw withdrawal responses did not differ between Burst- and Con-SCS. As the anti-nociceptive effect of Burst- and Con-SCS might be related to modulation of fundamentally different pain pathways, the use of operant testing methods, addressing cognitive and motivational aspects of pain, is highly recommended to further elucidate the behavioral differences between Burst-SCS and ConSCS. 


\section{References}

1. Slangen, R., et al., Spinal cord stimulation and pain relief in painful diabetic peripheral neuropathy: a prospective two-center randomized controlled trial. Diabetes Care, 2014. 37(11): p. 3016-24.

2. Kumar, K., et al., Spinal cord stimulation versus conventional medical management for neuropathic pain: a multicentre randomised controlled trial in patients with failed back surgery syndrome. Pain, 2007. 132(1-2): p. 179-88.

3. Geurts, J.W., et al., Spinal cord stimulation for complex regional pain syndrome type I: a prospective cohort study with long-term follow-up. Neuromodulation, 2013. 16(6): p. 523-9; discussion 529.

4. Kumar, K., M. Abbas, and S. Rizvi, The use of spinal cord stimulation in pain management. Pain Manag, 2012. 2(2): p. 125-34.

5. Kumar, K., S. Rizvi, and S.B. Bnurs, Spinal cord stimulation is effective in management of complex regional pain syndrome l: fact or fiction. Neurosurgery, 2011. 69(3): p. 566-78; discussion 5578-80.

6. Meyerson, B.A. and B. Linderoth, Mode of action of spinal cord stimulation in neuropathic pain. J Pain Symptom Manage, 2006. 31(4 Suppl): p. S6-12.

7. Shechter, R., et al., Conventional and kilohertz-frequency spinal cord stimulation produces intensity- and frequency-dependent inhibition of mechanical hypersensitivity in a rat model of neuropathic pain. Anesthesiology, 2013. 119(2): p. 422-32.

8. Taylor, R.S., et al., Predictors of pain relief following spinal cord stimulation in chronic back and leg pain and failed back surgery syndrome: a systematic review and meta-regression analysis. Pain Pract, 2014. 14(6): p. 489-505.

9. De Ridder, D., et al., Burst spinal cord stimulation for limb and back pain. World Neurosurg, 2013. 80(5): p. 642-649 e1.

10. De Ridder, D., et al., Burst spinal cord stimulation: toward paresthesia-free pain suppression. Neurosurgery, 2010. 66(5): p. 986-90.

11. De Ridder, D., et al., A 2-center comparative study on tonic versus burst spinal cord stimulation: amount of responders and amount of pain suppression. Clin J Pain, 2015. 31(5): p. 433-7.

12. Kriek, N., et al., Preferred frequencies and waveforms for spinal cord stimulation in patients with complex regional pain syndrome: A multicentre, double-blind, randomized and placebocontrolled crossover trial. Eur J Pain, 2016.

13. de Vos, C.C., et al., Burst spinal cord stimulation evaluated in patients with failed back surgery syndrome and painful diabetic neuropathy. Neuromodulation, 2014. 17(2): p. 152-9.

14. Crosby, N.D., et al., Stimulation parameters define the effectiveness of burst spinal cord stimulation in a rat model of neuropathic pain. Neuromodulation, 2015. 18(1): p. 1-8; discussion 8.

15. Crosby, N.D., et al., Burst and Tonic Spinal Cord Stimulation Differentially Activate GABAergic Mechanisms to Attenuate Pain in a Rat Model of Cervical Radiculopathy. IEEE Trans Biomed Eng, 2015. 62(6): p. 1604-13.

16. Gong, W.Y., L.M. Johanek, and K.A. Sluka, A Comparison of the Effects of Burst and Tonic Spinal Cord Stimulation on Hyperalgesia and Physical Activity in an Animal Model of Neuropathic Pain. Anesth Analg, 2016.

17. Meuwissen, K.P.V., et al., Conventional-SCS vs. Burst-SCS and the Behavioral Effect on Mechanical Hypersensitivity in a Rat Model of Chronic Neuropathic Pain: Effect of Amplitude. Neuromodulation, 2018. 21(1): p. 19-30.

18. Seltzer, Z., R. Dubner, and Y. Shir, A novel behavioral model of neuropathic pain disorders produced in rats by partial sciatic nerve injury. Pain, 1990. 43(2): p. 205-18. 
19. Truin, M., et al., Successful pain relief in non-responders to spinal cord stimulation: The combined use of ketamine and spinal cord stimulation. European Journal of Pain, 2011. 15(10).

20. Truin, M., et al., Increased efficacy of early spinal cord stimulation in an animal model of neuropathic pain. Eur J Pain, 2011. 15(2): p. 111-7.

21. Pluijms, W.A., et al., The effect of spinal cord stimulation frequency in experimental painful diabetic polyneuropathy. Eur J Pain, 2013. 17(9): p. 1338-46.

22. van Beek, M., et al., Spinal cord stimulation in experimental chronic painful diabetic polyneuropathy: Delayed effect of High-frequency stimulation. Eur J Pain, 2017. 21(5): p. 795803.

23. Chaplan, S.R., et al., Quantitative assessment of tactile allodynia in the rat paw. J Neurosci Methods, 1994. 53(1): p. 55-63.

24. Smits, H., et al., Effect of spinal cord stimulation in an animal model of neuropathic pain relates to degree of tactile "allodynia". Neuroscience, 2006. 143(2): p. 541-6.

25. Smits, H., M. van Kleef, and E.A. Joosten, Spinal cord stimulation of dorsal columns in a rat model of neuropathic pain: evidence for a segmental spinal mechanism of pain relief. Pain, 2012. 153(1): p. 177-83.

26. Meyerson, B.A., et al., Spinal cord stimulation in animal models of mononeuropathy: effects on the withdrawal response and the flexor reflex. Pain, 1995. 61(2): p. 229-43.

27. De Ridder, D. and S. Vanneste, Response: A Systematic Evaluation of Burst Spinal Cord Stimulation for Chronic Back and Limb Pain. Neuromodulation, 2016. 19(7): p. 785-786.

28. Mills, C., et al., Estimating efficacy and drug ED50's using von Frey thresholds: impact of weber's law and log transformation. J Pain, 2012. 13(6): p. 519-23.

29. Flor, H., New developments in the understanding and management of persistent pain. Curr Opin Psychiatry, 2012. 25(2): p. 109-13.

30. Stancak, A., et al., Functional magnetic resonance imaging of cerebral activation during spinal cord stimulation in failed back surgery syndrome patients. Eur J Pain, 2008. 12(2): p. 137-48.

31. Pope, J.E., S. Falowski, and T.R. Deer, Advanced waveforms and frequency with spinal cord stimulation: burst and high-frequency energy delivery. Expert Rev Med Devices, 2015. 12(4): p. 431-7.

32. Yearwood, T.L., et al., Pulse width programming in spinal cord stimulation: a clinical study. Pain Physician, 2010. 13(4): p. 321-35.

33. De Ridder, D., S. Perera, and S. Vanneste, Are $10 \mathrm{kHz}$ Stimulation and Burst Stimulation Fundamentally the Same? Neuromodulation, 2017. 20(7): p. 650-653.

34. Gao, J., et al., Effects of spinal cord stimulation with "standard clinical" and higher frequencies on peripheral blood flow in rats. Brain Research, 2010. 1313: p. 53-61.

35. Zhang, T.C., J.J. Janik, and W.M. Grill, Modeling effects of spinal cord stimulation on widedynamic range dorsal horn neurons: influence of stimulation frequency and GABAergic inhibition. J Neurophysiol, 2014. 112(3): p. 552-67.

36. Cui, J.G., et al., Spinal cord stimulation attenuates augmented dorsal horn release of excitatory amino acids in mononeuropathy via a GABAergic mechanism. Pain, 1997. 73(1): p. 87-95.

37. Tang, R., et al., Comparison of burst and tonic spinal cord stimulation on spinal neural processing in an animal model. Neuromodulation, 2014. 17(2): p. 143-51.

38. Moens, M., et al., Spinal cord stimulation modulates cerebral function: an fMRI study. Neuroradiology, 2012. 54(12): p. 1399-407.

39. Mogil, J.S., Animal models of pain: progress and challenges. Nat Rev Neurosci, 2009. 10(4): p. 283-94.

40. Woolf, C.J., Long term alterations in the excitability of the flexion reflex produced by peripheral tissue injury in the chronic decerebrate rat. Pain, 1984. 18(4): p. 325-43.

41. Matthies, B.K. and K.B. Franklin, Formalin pain is expressed in decerebrate rats but not attenuated by morphine. Pain, 1992. 51(2): p. 199-206. 
Chapter 3

42. Harte, S.E., et al., Mechanical Conflict System: A Novel Operant Method for the Assessment of Nociceptive Behavior. PLoS One, 2016. 11(2): p. e0150164. 


\section{CHAPTER 4}

\section{Burst and Tonic Spinal Cord Stimulation Both Activate Spinal GABAergic Mechanisms to Attenuate Pain in a Rat Model of Chronic Neuropathic Pain.}

Koen P.V. Meuwissen, Luuk E. de Vries, Tianhe C. Zhang, Jianwen Wendy Gu, Elbert A. J. Joosten.

Accepted in Journal of Pain Practice

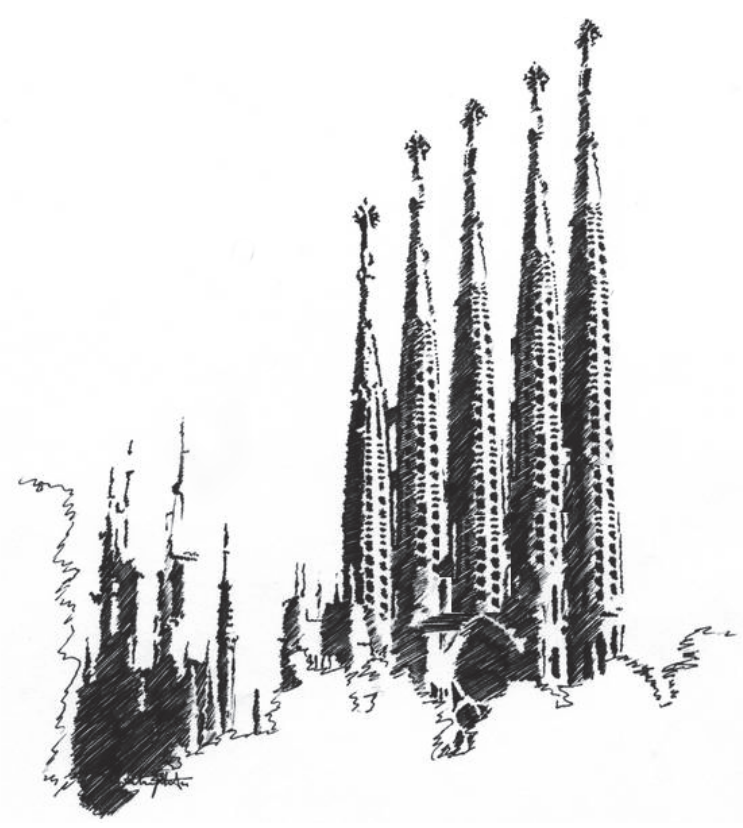




\begin{abstract}
Background: Experimental and clinical studies have shown that Tonic Spinal Cord Stimulation (SCS) releases GABA in the spinal dorsal horn. Recently, it was suggested that Burst SCS does not act via spinal GABAergic mechanisms. Therefore, we studied spinal GABA-release during Burst and Tonic SCS, both anatomically and pharmacologically, in a well-established chronic neuropathic pain model.
\end{abstract}

Methods: Animals received a partial sciatic nerve ligation (PSNL). Quantitative immunohistochemical (IHC) analysis of intracellular GABA-levels in the lumbar L4-L6 dorsal spinal cord was performed after 60 min of Burst, Tonic or Sham SCS in PSNL rats $(n=16)$. In a second pharmacological experiment the effect of intrathecal administration of the $G A B A_{A^{-}}$ antagonist bicuculline $(5 \mu \mathrm{g})$ and the $\mathrm{GABA}_{\mathbf{B}}$-antagonist phaclofen $(5 \mu \mathrm{g})$ was assessed. Paw withdrawal thresholds to von Frey filaments of PSNL-rats $(n=20)$ were tested during 60 minutes of Burst and Tonic SCS, 30 mins post intrathecal administration of the drugs.

Results: Quantitative IHC-analysis of GABA-immunoreactivity (IR) in spinal dorsal horn sections of animals that had received Burst SCS $(n=5)$ showed significantly lower intracellular GABA-levels, when compared to Sham SCS sections $(n=4)(p=0.0201)$ and Tonic SCS sections $(n=7)(p=0.0077)$. Intrathecal application of $\mathrm{GABA}_{A}$-antagonist bicuculline $(5 \mu \mathrm{g})(n=10)$ or the $\mathrm{GABA}_{B}$-antagonist phaclofen $(5 \mu \mathrm{g})(\mathrm{n}=10)$ resulted in ablation of the analgesic effect for both Burst SCS and Tonic SCS.

Conclusions: In conclusion, our anatomical and pharmacological data demonstrate that, in this well-established chronic neuropathic animal model, the analgesic effect of both Burst SCS and Tonic SCS is mediated via spinal GABAergic mechanisms. 


\section{Introduction}

Tonic Spinal Cord Stimulation (SCS) is a last-resort treatment method for patients that suffer from intractable chronic neuropathic pain [1-6]. The standard Tonic SCS-protocol consists of continuous, tonic, electrical stimulation applied to the dorsal columns of the spinal cord with a frequency within the range of $40-80 \mathrm{~Hz}$ and a pulse width in between $200-500 \mu \mathrm{Sec}$ $[2,7,8]$. The concept of SCS emerged as a direct application of the 'Gate Control Theory', by Melzack and Wall in 1965 [7, 9]. It was postulated that antidromic stimulation of the nonnociceptive $A \beta$-fibers could close the 'spinal gate', located in the dorsal horn of the spinal cord. Closing of the 'gate' is facilitated by inhibitory interneurons located in the superficial laminae of the dorsal horn and it is believed that the neurotransmitter $\gamma$-aminobutyric acid (GABA) plays a pivotal role in this process [8, 10,11]. During the development of neuropathic pain Janssen and colleagues observed, in a partial sciatic nerve ligation (PSNL)model, increased intracellular levels of GABA in the dorsal horn [12]. Later, it was demonstrated that Tonic SCS decreased intracellular GABA-immunoreactivity in the dorsal horn of these PSNL-rats [13]. At the same time, extensive experimental microdialysis-work has demonstrated that Tonic SCS increases extracellular GABA levels in the dorsal horn of allodynic PSNL-rats [14-16]. Thus, GABAergic interplay seems to be an important aspect of the analgesic mechanisms underlying Tonic SCS in the PSNL-model of chronic neuropathic pain. The role of GABA in segmental SCS-mechanisms was further elucidated by the administration of pharmacological agents that specifically modulate GABA-release in the dorsal horn during Tonic SCS in PSNL-neuropathic rats. Local perfusion with a $G_{A B A_{B}-}$ receptor antagonist in the dorsal horn transiently abolished the SCS-induced effect in neuropathic rats [16] and rats not receiving adequate pain relief with Tonic SCS (nonresponders) were turned into responders by administration of the $\mathrm{GABA}_{B}$-receptor agonist Baclofen [17]. The aforementioned preclinical findings were successfully translated to the clinic where neuropathic pain patients with a deficient Tonic SCS effect had improved pain relief following the intrathecal administration of baclofen [18], further confirming the theory that local spinal GABAergic mechanisms are pivotal for the effects of Tonic SCS. While GABA plays a role in the underlying mechanism of Tonic SCS, the neurotransmitters involved in other stimulation paradigms, such as Burst SCS, have not been clearly identified. As patients show different responses to different stimulation paradigms, the underlying mechanisms may be different [19]. The Burst waveform consists of closely spaced pulses delivered in a packet or burst, directly followed by a quiescent period or interburst interval [20]. Burst SCS has proven to be effective in FBSS and Radiculopathy patients, and clinical 
trials have demonstrated its ability to help patients to reduce their analgesic intake [21-23]. In addition, Burst SCS reduces neuropathic pain without generating paresthesia in the affected limb or area [24-26]. Yet, from a mechanistic point of view, the Burst waveform is still in its infancy. Source-localized EEG-studies and patient questionnaires suggest that Burst SCS preferentially activates the medial pain pathway, hence modulates emotionalaffective pain aspects $[25,27]$. On a segmental level, an experimental electrophysiological study has aimed to elucidate the involvement of GABA in the spinal mechanism underlying

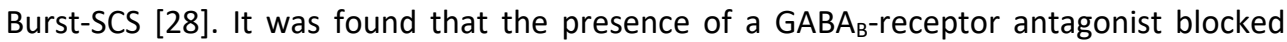
attenuation of dorsal horn neuronal firing during Tonic SCS but not Burst SCS. Furthermore, blood serum GABA measurements showed that systemic GABA levels were not increased following Burst SCS. From this, the authors concluded that Burst SCS might not act via spinal GABAergic mechanisms [31]. However, it should be mentioned that the aforementioned study was performed not only in an uncommon rat model for chronic neuropathic pain, the Painful Cervical Nerve Root Compression model, but these experiments were also terminal and thus no behavioral testing during the conscious state of the animals was performed [31]. This makes interpretation of these data in the context of understanding the role of spinal GABA in a chronic neuropathic pain model difficult, as most experimental data on pain relief and the spinal GABAergic mechanism underlying Tonic SCS have been documented and studied in the well-described and validated partial sciatic nerve ligation (PSNL) model or a similar nerve injury model for peripheral mononeuropathy [13-17, 29]. Therefore, in order to further understand the spinal mechanism underlying Burst-SCS we aimed to study, both anatomically and pharmacologically, the role of GABA in behavior and pain relieving mechanisms underlying Burst and Tonic SCS, in a well-established chronic neuropathic PSNL model. 


\section{Material and Methods}

\section{Ethics Statement}

The experiments were performed in accordance with the European Directive for the Protection of Vertebrate Animals Used for Experimental and Other Scientific Purposes (86/609/EU). The protocol was approved by the Animal Research Committee of the Maastricht University Medical Centre (Project License number: 2017-022).

\section{Animals}

All experiments were performed using male Sprague Dawley rats $(n=36$, which were youngadult ( 5 weeks of age) at the start of the experiment (150-200 g). Sixteen animals were used in the first experiment in which we assessed the quantitative immunohistochemical (IHC)analysis of GABA- immunoreactivity (IR) in spinal dorsal horn sections of animals after SCS $(n=16)$. Twenty animals were used in the second experiment in which we intrathecally

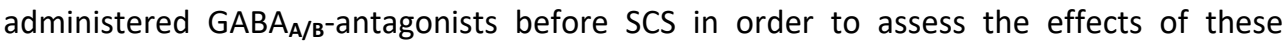
pharmacological agents on behavioral pain relieving effects of SCS $(n=20)$. Animals were housed in groups of 2 , in polycarbonate cages in a climate-controlled vivarium maintained under controlled temperature $\left(21 \pm 1^{\circ} \mathrm{C}\right)$, relative humidity $(55 \pm 15 \%)$ and artificial lighting (12:12 reversed light/dark cycle) with distilled water and rodent food available ad libitum. The vivarium was equipped with a central radio system, continuously producing background music at 45 decibel, in order to desensitize the animals for experimenter related noise. All procedures were conducted between 09:00 and 16:00 hours. Experiments were conducted during the dark, active, phase of the rodent circadian rhythm.

\section{Partial Sciatic Nerve Ligation (PSNL)}

A unilateral ligation of the left sciatic nerve was performed as described by Seltzer et al. 1990 [29], and previously applied in our laboratory [30-33]. In short, animals were anesthetized with 3-5\% isoflurane (Abbott Laboratories Ltd., Kent, U.K.) and air enriched with $100 \%$ oxygen at a constant flow rate of $250 \mathrm{ml} / \mathrm{min}$. By use of an automatic heating pad body temperature was maintained at $37.5^{\circ} \mathrm{C}$. The nervus ischiadicus from the left hind paw was exposed by blunt dissection and carefully freed from surrounding connective tissue. Subsequently, the nerve was partially (approximately $1 / 3$ ) ligated using $8 / 0$ nonabsorbable silk suture. The wound was then closed with $4 / 0$ silk sutures. Development of mechanical hypersensitivity (mechanical allodynia) was monitored with the use of von Frey assays for 14 consecutive days. At day 14, presence of mechanical hypersensitivity was 
confirmed if the log (50\% paw withdrawal threshold (PWT)) was decreased by 0.2 units compared to baseline (day 0) [34].

\section{Assessment of mechanical hypersensitivity (von Frey assay)}

Mechanical hypersensitivity was assessed by use of von Frey filaments. Von Frey assessment was always conducted in the same room, isolated from external sounds and equipped with artificial red light sources (temperature: $21 \pm 1^{\circ} \mathrm{C}$, and relative humidity $: 55 \pm 15 \%$ ). Prior to testing, animals were placed in the behavioral set-up for 15 mins in order to acclimate to the new surroundings. The assessment-room was equipped with a mobile radio, continuously producing background music at 45 decibels. PWTs to von Frey filaments were assessed using the 'up-down method' [35]. Von Frey filaments of linearly incrementing stiffness (bending forces 0.6, 1.2, 2.0, 3.6, 5.5, 111 8.5, 15.1g and $28.84 \mathrm{~g}$ ) were applied to the plantar surface of the hind paw of the rats for 5 consecutive seconds. A negative response (the hind paw was not withdrawn) was followed by the subsequent filament with greater bending force. In contrast, a positive response (the hind paw was withdrawn) was followed by application of the previous filament with a lower bending force. After completion of a sequence of six consecutive responses the 50\% PWT was calculated [35]. The predetermined cut-off value was set at $28.84 \mathrm{~g}$. For statistical analysis the $50 \%$ PWTs were logarithmically transformed to yield a linear scale.

\section{Tissue preparation}

For immunohistochemistry experiments, sixteen animals in experiment 1 were divided into three groups: sham SCS ( $=4)$ and Tonic SCS $(n=7)$ and Burst SCS $(n=5)$. Animals were sacrificed 60 mins after the start of (sham-) SCS. Tissue perfusion was performed transcardially with a mixture of $4 \%$ paraformaldehyde and $15 \%$ picric acid in $0.2 \mathrm{M}$ phosphate buffer (PBS; pH 7.6) after anesthesia with pentobarbital (100 mg/kg body weight). Then, lumbar spinal cord regions L4-L6 were removed by a laminectomy, postfixated overnight at $4^{\circ} \mathrm{C}$ and cryoprotected for $24 \mathrm{~h}$ in $10 \%$ sucrose. This was followed by at least $72 \mathrm{~h}$ incubation in $25 \%$ sucrose (in $0.1 \mathrm{M} \mathrm{PBS} ; \mathrm{pH} 7.6$ ) at $4^{\circ} \mathrm{C}$. Subsequently, tissues were frozen in solid carbon dioxide. Thirty $\mu \mathrm{m}$ thick transverse cryosections were mounted on gelatine-coated glass slides and stored at $-20^{\circ} \mathrm{C}$ until staining procedures were performed. 


\section{Immunohistochemical detection of GABA}

The immunohistochemistry-protocol was performed as described in Janssen et al. (2012) [13]). In short, staining procedures were performed at room temperature unless stated otherwise. Firstly, glass slides were air-dried for 2 hours and subsequently washed in Trisbuffered saline (TBS, $0.1 \mathrm{M}, \mathrm{pH}$ 7.6) including 0.3\% Triton X-100 (TBS-T), TBS and TBS-T. Blocking was performed with $2 \%$ normal donkey serum (Sigma-Aldrich, Zwijndrecht, The Netherlands). The serum was diluted in TBS-T and applied for a 1-h incubation period. Then, the sections were incubated with a polyclonal rabbit anti-GABA antibody (1:5000 diluted in TBS-T; Sigma- Aldrich, Zwijndrecht, The Netherlands, A2052) for a time period of 48 hours. After 48 hours, excess of primary antibody was removed by use of TBS after which sections were incubated with the secondary alexa fluor 488 donkey anti-rabbit IgG antibody (1:100 diluted in TBS-T; Invitrogen, Breda, The Netherlands, A21206) for 2 hours. Subsequently, sections were rinsed with TBS, and coverslipped with TBS/glycerol (20\%/80\%).

\section{Quantification of immunostaining}

Quantitative immuhistochemical analysis of spinal dorsal horn GABA staining was performed as as described by Janssen et al. $(2012)[12,13]$. Photomicrographs were taken of both ipsi- and contralateral lumbar L4-L6 spinal cord immunostained sections using a Provis AX70 fluorescent microscope (Olympus, Hamburg, Germany). The microscope was connected to a digital black and white video camera (U-CMAD-2, Olympus), equipped with CellPC software. Lamina of Rexed 1-3 of the dorsal horn were determined as regions of interest for the GABA-IR analysis [36]. Grayscale values were calculated for these laminae. Analysis of gray scale spinal cord pictures was performed by a blinded observer by use of the AnalySIS software program CellP@ (Soft Imaging Systems, Münster, Germany).

\section{Implantation of spinal cord stimulation device}

The implantation of the SCS device was performed according to the standard protocol used in our institution [31-33, 37, 38]. In short, a small laminectomy was made at level Thoracic 13 (Th13), after which the spinal cord was exposed by use of a surgical rotary tool. A custommade cylindrical 4-contact lead $(0.72 \mathrm{~mm}$ diameter; Boston Scientific Neuromodulation, Valencia, CA, USA) was inserted into the epidural space, in the caudal direction. Electrode configuration was set at alternating cathode and anode settings (rostral to caudal: + - + -). Then, the electrode was secured to a spinous process with tissue adhesive (Histoacryl ${ }^{\circledR}, \mathrm{B}$ Braun Medical BV, Oss, the Netherlands) to prevent electrode migration. The electrode wires were tunneled subcutaneously to the neck of the animal and the stimulator- 
connectors were attached with $4 / 0$ silk sutures. Animals were given 2 days for recovery prior to the initiation of SCS-experiments.

\section{Intrathecal implantation}

After successful implantation of the SCS device twenty animals in experiment 2 received a small laminectomy was made at level Thoracic 12 (Th12), after which the spinal cord was exposed by use of a surgical rotary tool. The membrane was carefully opened after which a polyethylene catheter from Instech Laboratories (27ga, length $1.5 \mathrm{~cm}$ ) was inserted and tunneled intrathecally to the level of the L3-L5 spinal segments as described by Truin et al. (2011) [30]. The catheter was secured to a spinous process with tissue adhesive (Histoacryl ${ }^{\circledR}$, B Braun Medical BV, Oss, the Netherlands) to prevent migration. Subsequently, the catheter was slowly flushed with $10 \mu \mathrm{l}$ of saline and the wound was closed with 4/0 silk sutures. Animals with signs of paralysis directly after the surgery were excluded. Correct placement of the catheter was confirmed when lidocaine $2 \%$ injection (10 $\mathrm{mg} / \mathrm{ml}$ ) resulted in paralysis or dragging of the hind limbs [30].

\section{Spinal cord stimulation}

For stimulation of the dorsal columns an A-M systems stimulator (MultiStim: Programmable 8-Channel Stimulator (Model 3800) $220 \mathrm{~V} / 50 \mathrm{~Hz}$ ) fitted with a stimulus Isolator (Model 3820 for A-M Systems MultiStim) was used. The stimulator was set to deliver constant current biphasic stimulation for both the Tonic and Burst SCS modes. Tonic SCS Motor Threshold (MT) was determined at the following settings: pulse width of $200 \mu \mathrm{S}$ administered at a frequency of $2 \mathrm{~Hz}$. Burst SCS MT was determined at the following settings: pulse width of $1000 \mu \mathrm{S}$, five pulses ( $449 \mathrm{~Hz}$ intra-burst frequency) administered at an inter-burst frequency of $2 \mathrm{~Hz}$. The amplitude was gradually increased until symmetrical contractions of the hind limbs were perceived by hand and/or visually observed. Then, either a Tonic SCS paradigm at $66 \%$ of MT (frequency $50 \mathrm{~Hz}$, pulse width $200 \mu \mathrm{S}$ ) [20], or a biphasic Burst-SCS paradigm at $50 \% \mathrm{MT}$ (inter-burst-frequency $40 \mathrm{~Hz}$, pulse width $1000 \mu \mathrm{S}, 5$ spikes at $449 \mathrm{~Hz}$ intra-burst frequency) was applied for 60 minutes [24, 33]. The SCS-parameters were based on previously determined optimal settings for pain relief with Burst SCS (50\% MT) and Tonic SCS $(66 \% \mathrm{MT})[31,33]$. In all experiments, the PWTs to von Frey filaments were assessed before the start of SCS treatment (baseline), at 15', 30', 45' and 60' min. after stimulation was turned on. The investigator was blinded to the stimulation condition throughout the whole experiment. 


\section{Intrathecal administration of $\mathrm{GABA}_{\mathrm{A} / \mathrm{B}}$ Receptor Antagonist (experiment 2)}

The concentration of the $\mathrm{GABA}_{\mathrm{A} / \mathrm{B}}$-receptor antagonists bicuculline $(5 \mu \mathrm{g})(\geq 97 \%$ (NMR); Sigma-Aldrich) and phaclofen ( $5 \mu \mathrm{g})$ ( $\geq 97 \%$ (NMR); Sigma-Aldrich) was chosen based on literature demonstrating a dose-response curve for both antagonists [39]. As the peak dorsal horn drug concentrations occurred after $30 \mathrm{~min}$ after intrathecal administration [39], antagonists were applied to the spinal cord 30 mins before the SCS and von Frey testing protocols were initiated. Intrathecal administration of the $\mathrm{GABA}_{\mathrm{A} / \mathrm{B}}$-receptor antagonists $(5 \mu \mathrm{g})$ was followed by the administration of $20 \mu \mathrm{l}$ saline. Vehicle administration consisted of $10 \mu \mathrm{l}$ saline followed by the administration of $20 \mu \mathrm{l}$ saline.

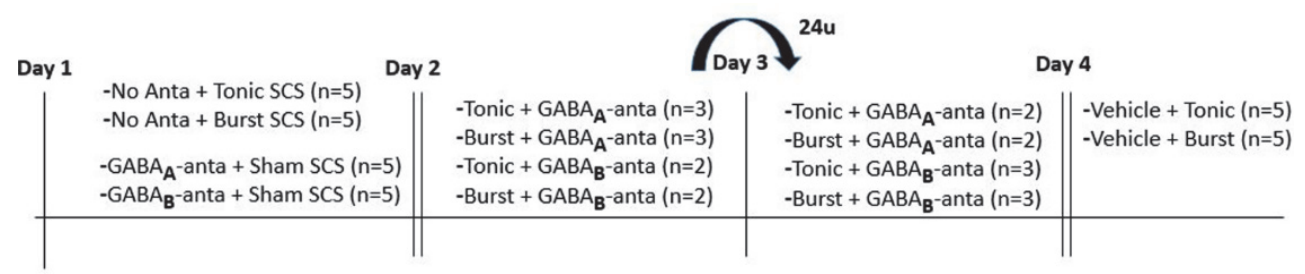

Fig.1: Timeline of experiment 2: pharmacological intrathecal experiments.

\section{Data Analysis}

PWT's to von Frey filaments are presented as mean \pm standard error of the mean (SEM). In line with previous Von Frey studies [32, 33, 40], von Frey data were logarithmically transformed to obtain a linear scale and to account for Weber's Law [41]. For statistical analysis of differences in the withdrawal thresholds over time within groups of experiment 1 and 2, the non-parametric Friedman test was used followed by followed by Dunn's post hoc test. For the analysis of differences in the withdrawal thresholds between groups, ipsilateral and contralateral withdrawal thresholds, the nonparametric Mann-Whitney- $U$ test was used. Before statistical analysis of GABA-IR gray values in experiment 1 , a ShapiroWilk normality test was performed. Then, the Kruskal-Wallis test was performed in order to assess statistical differences over group means. Dunn's Multiple Comparison Test followed this in order to assess significant differences between the group means. All statistical analyses were performed with $\alpha=0.05$ using IBM SPSS statistics 23 . 


\section{Results}

\section{Development of mechanical hypersensitivity (von Frey), and SCS}

In experiment 1, after PSNL-surgery, ipsilateral hindpaw paw withdrawal thresholds (PWTs) were significantly lower than ipsilateral hindpaw PWTs in experiment 1 ( $p=0.019)$; all 16 animals qualified as hypersensitive due to increased response to mechanical stimulation by von Frey filaments (ipsilateral average PWTs: $11.5 \pm 0.9 \mathrm{~g}$ (pre-lesion) vs. $1.1 \pm 0.5 \mathrm{~g}$ (postlesion); $\mathrm{p}=0.0042$ ) (see Methods) (Fig.2A). Burst SCS ( $n=5)$, significantly increased PWTs compared to baseline PWTs at $15(p=0.046), 30(p=0.025), 45(p=0.016)$, and 60 mins of $\operatorname{SCS}(p=0.019)$ (Fig.3A). For Tonic SCS $(n=7)$, PWTs significantly differed from baseline PWTs at $15(p=0.027), 30(p=0.026), 45(p=0.033)$, and 60 mins of $\operatorname{SCS}(p=0.031)$ (Fig.3A). Sham SCS $(n=4)$ did not increase PWTs at any time points (Fig.3A).
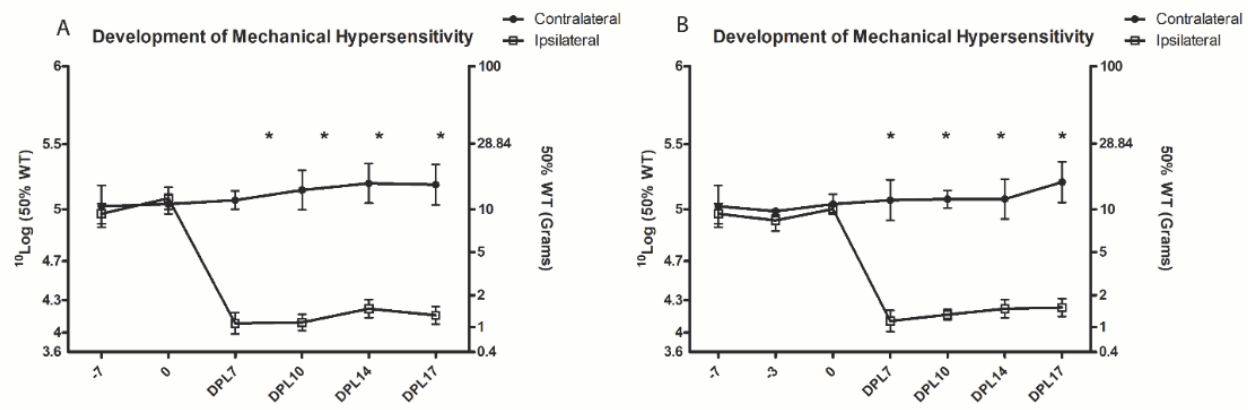

Fig.2 A) Development of mechanical hypersensitivity for experiment 1 ( $n=16)$ B) Development of mechanical hypersensitivity for experiment $2(n=20) .{ }^{*} p<0.05$ for ipsilateral versus contralateral PWT's. DPL=Days Post Ligation.

In experiment 2 (see methods), after PSNL-surgery, ipsilateral hindpaw PWTs were significantly lower than ipsilateral hindpaw PWTs $(p=0.023)$ : all 20 animals qualified as hypersensitive (ipsilateral average PWTs: $10.9 \pm 1.1 \mathrm{~g}$ (pre-lesion) vs. $1.3 \pm 0.7 \mathrm{~g}$ (post-lesion); $\mathrm{p}=0.0064$ ) (see Methods) (Fig.2B). For Burst SCS ( $n=10)$, PWTs significantly differed from baseline PWTs at $15(p=0.041), 30(p=0.027), 45(p=0.028)$, and 60 mins. of SCS $(p=$ 0.022 ) (Fig.3B). For Tonic SCS ( $n=10)$, PWTs significantly differed from baseline PWTs at 15 ( $p=0.029), 30(p=0.028), 45(p=0.031)$, and 60 mins of SCS ( $p=0.035)$ (Fig.3B). No significant differences were reported between development of mechanical hypersensitivity in experiment 1 and 2. In addition, no significant differences were reported between SCS 
time points in experiment 1 and 2 . When SCS-data of experiment 1 and 2 were pooled, Burst SCS ( $n=15)$ and Tonic SCS ( $n=17)$, significantly increased PWTs compared to baseline PWTs at 15 ( $p=0.042 ; p=0.028$ resp.), 30 ( $p=0.027 ; p=0.026$ resp.), 45 ( $p=0.021 ; p=0.032$ resp.), and 60 mins of stimulation ( $p=0.025 ; p=0.033$ resp.) (Fig.3C). Burst SCS PWTs and Tonic SCS PWTs significantly differed at 15 mins of SCS ( $p=0.038$ ) (Fig.3C).
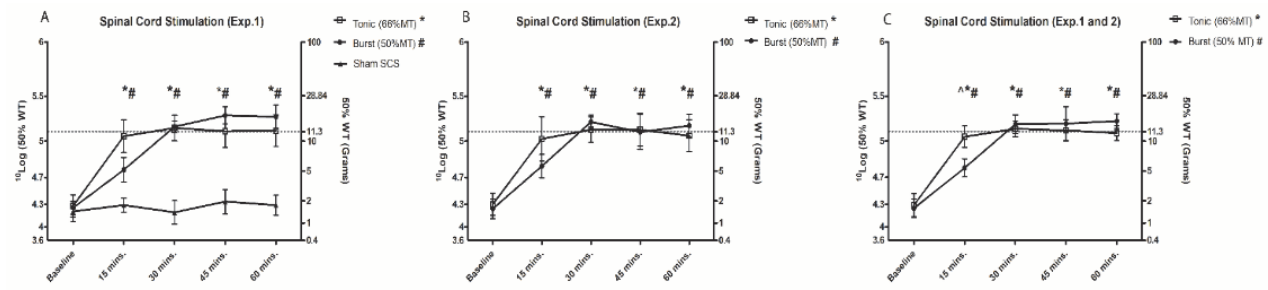

Fig.3 A) The effect of Tonic SCS ( $n=7)$, Burst SCS $(n=5)$ and Sham SCS $(n=4)$ on PWT's based on sensitivity to von Frey filaments in experiment 1. B) The effect of Tonic SCS $(n=10)$ and Burst SCS $(n=10)$ on PWT's in experiment 2. C) The effect of Tonic SCS ( $n=17)$, Burst SCS ( $n=15)$ on PWT's pooled data from experiment 1 and experiment 2. The dotted line represents the average PWT baseline prior to sciatic nerve ligation. ( $~ p<0.05$ for Burst-SCS vs. Tonic SCS; \# $p<0.05$ for Burst SCS vs. baseline; * $p<0.05$ for Tonic SCS vs. baseline).

\section{Spinal DH GABA-immunoreactivity}

In experiment 1 , anti-GABA immunohistochemistry revealed an intense immunoreactivity (IR) predominantly in laminae 1-3 of the lumbar spinal dorsal horn with clear identification of GABA-IR cell bodies (Fig. 4B). Grayscale values were calculated for these laminae. The Shapiro-Wilk normality test was not passed $(p=0.0395)$. Accordingly, a Kruskal-Wallis test was performed which showed a significant difference between mean gray values for the Sham-SCS, Tonic-SCS and Burst SCS group ( $p=0.0085, \mathrm{KW}$-statistic=9.524). Statistical testing of GABA staining intensity in spinal $\mathrm{DH}$ and gray values revealed that the Tonic SCS group, although it showed a strong tendency, did not differ significantly from the Sham-SCS group ( $p=0.1609$ ), the Burst SCS group differed significantly from the Sham-SCS group ( $p=$ $0.0201)$, and from the Tonic SCS group ( $p=0.0077)$. 
A

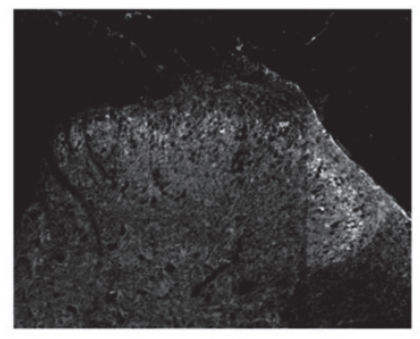

B

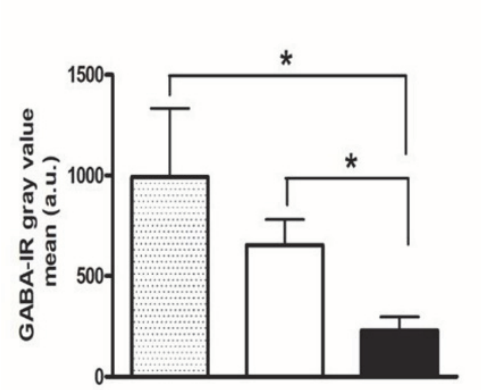

Tonic SCS
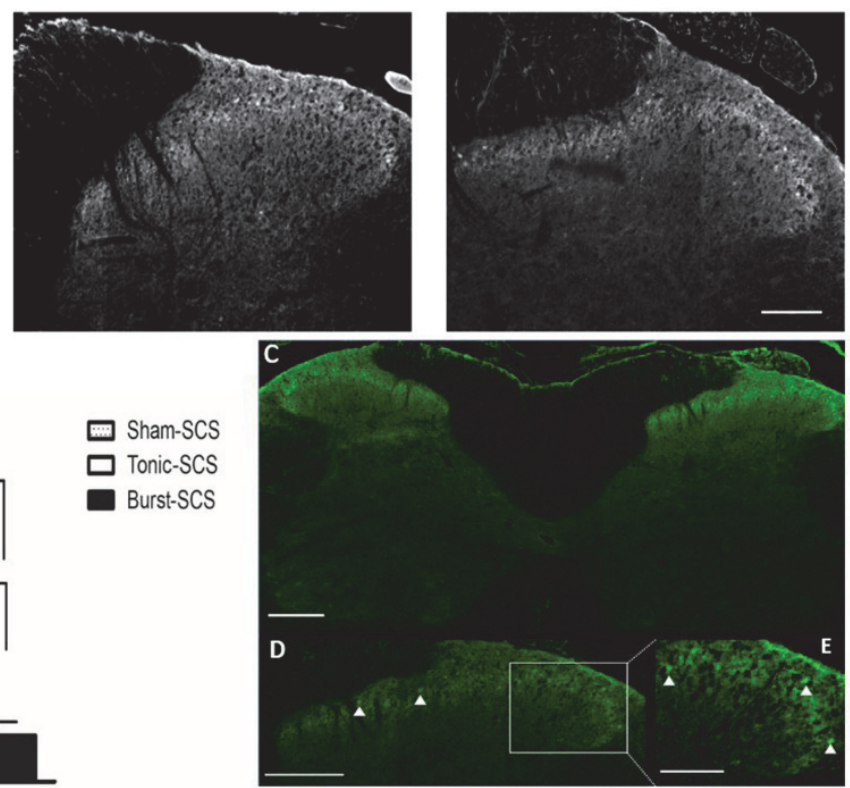

Fig.4: A) SCS-induced alterations in intracellular GABA levels in the L4-L6 spinal dorsal horn. Representative grayscale photomicrographs of the upper laminae of the ipsilateral dorsal horn of a sham SCS- animal, Tonic SCS animal, and Burst SCS animal. Scale bar $=100 \mu \mathrm{m}$. B) Quantified GABAIR in laminae 1-3 of the spinal dorsal horn L4-L6 of sham SCS animals $(n=4)$, Tonic SCS ( $n=7)$ animals and Burst SCS $(n=5)$ animals. * $p<0.05$. C) Representative overview of the immunostaining of the left and right dorsal horn of the spinal cord. Scale bar $=100 \mu \mathrm{m}$. D) Arrowheads point out activated GABAIR neuronal profiles, Scale bar $=100 \mu \mathrm{m}$. E) Scale bar $=50 \mu \mathrm{m}$. 


\section{Intrathecal GABA-antagonist administration}

In experiment 2 (Fig.1), thresholds for mechanical hypersensitivity were not affected after 15 mins of Tonic SCS with intrathecal administration of the $\mathrm{GABA}_{A}$-antagonist bicuculline, as PWT's were significantly increased as compared to baseline ( $p=0.044)$ ( (Fig.5A). Meanwhile, at 30 mins SCS $(p=0.38), 45$ mins SCS $(p=0.65)$, and 60 mins $(p=0.32)$ of Tonic SCS, thresholds for mechanical hypersensitivity were affected as PWTs did not significantly differ from baseline PWTs. Administration of the $G_{A B A}$-antagonist bicuculline affected thresholds for mechanical hypersensitivity at all time points during Burst SC. PWWs did not significantly differ from baseline PWTs at 15 mins $(p=0.74), 30$ mins $(p=0.49), 45$ mins ( $p$ $=0.52)$, and 60 mins of Burst SCS $(p=0.67)$ (Fig.5A).

After intrathecal administration of the $G_{A B A}$-antagonist phaclofen thresholds for mechanical hypersensitivity did not differ from baseline for both Tonic SCS and Burst SCS (Fig.5B). PWTs at 15 mins SCS ( $p=0.35, p=0.38$ resp.), 30 mins SCS ( $p=0.56, p=0.41$ resp.), 45 mins SCS ( $p=0.43, p=0.50$ resp.), and 60 mins of SCS ( $p=064, p=0.59$ resp.) did not significantly differ from baseline PWTs (Fig.5B).

In a control experiment, administration of vehicle (saline-minus antagonist) was administered. Thresholds for mechanical hypersensitivity were not affected as PWT's significantly increased over time for both Tonic-SCS $(n=10)$ and Burst-SCS $(n=10)$ at 15 mins ( $p=0.01, p=0.01$ resp.), 30 mins ( $p=0.02, p=0.01$ resp.), 45 mins $(p=0.01, p=0.03$ resp.), and 60 mins ( $p=0.01, p=0.01$ resp.), as compared to baseline (Fig.5C).

Administration of the $\mathrm{GABA}_{A}$-antagonist bicuculline $(n=5)$ and the $\mathrm{GABA}_{B}$-antagonist phaclofen $(n=5) 30$ minutes prior to Sham SCS did not increase PWTs, as compared to Baseline PWT's (Fig.5D).

Administration of the $\mathrm{GABA}_{A}$-antagonist bicuculline $(n=5)$ and the $\mathrm{GABA}_{B}$-antagonist phaclofen $(n=5) 30$ minutes prior to Von Frey testing decreased PWTs of the contralateral paw (unaffected by the sciatic nerve ligation) at 15 mins ( $p=0.01, p=0.01$ resp.), 30 mins ( $p=0.02, p=0.01$ resp.), 45 mins ( $p=0.01, p=0.03$ resp.), and 60 mins $(p=0.01, p=0.01$ resp.), of Von Frey testing, as compared to Minus 30 Baseline PWTs (Fig.6). 


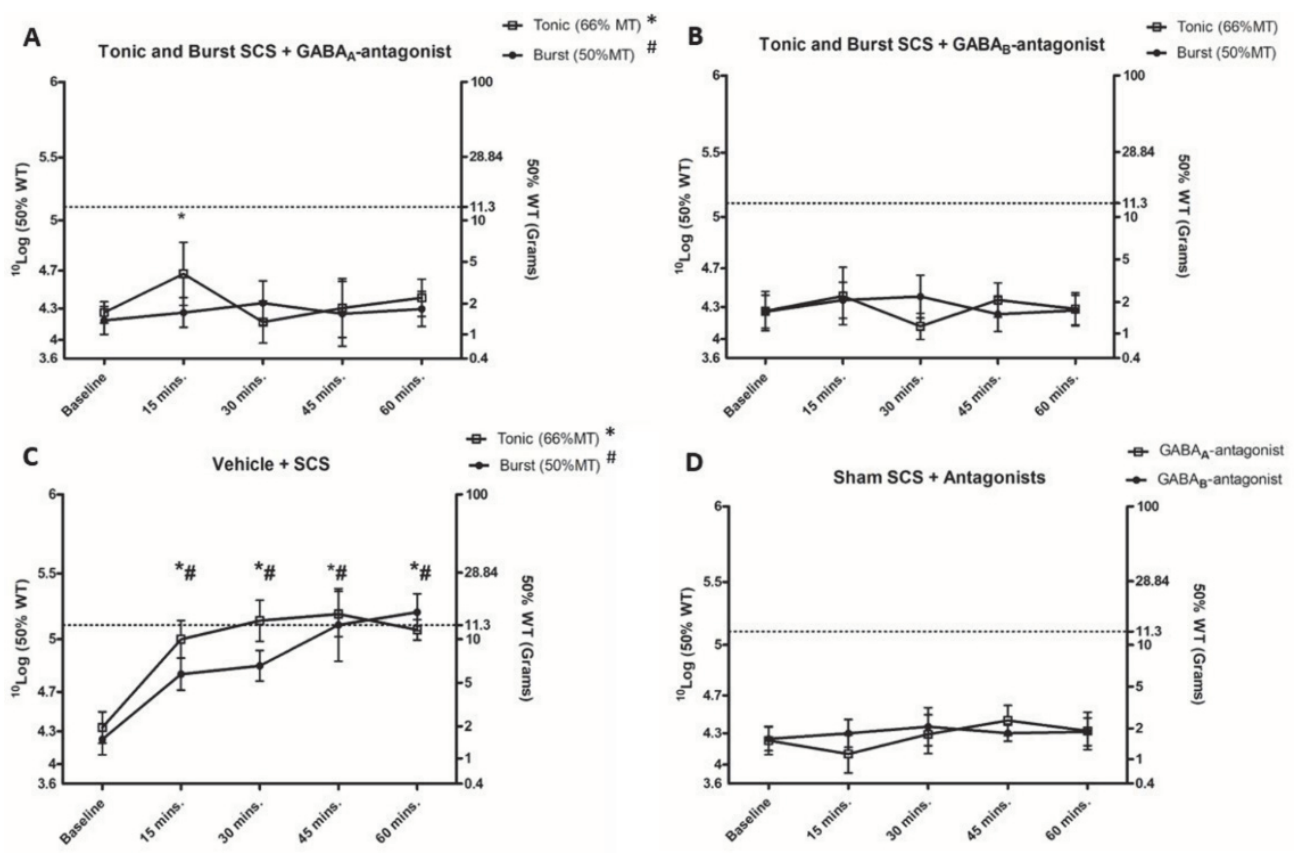

Fig.5: A) The effect of Tonic-SCS $(n=10)$ and Burst-SCS $(n=10)$ on the paw withdrawal thresholds (PWT's) based on sensitivity to von Frey filaments, 30 mins post GABA $A_{A}$-antagonist bicuculline administration. PWT's were assessed at baseline, 15, 30, 45, and 60 mins. of SCS. B) The effect of

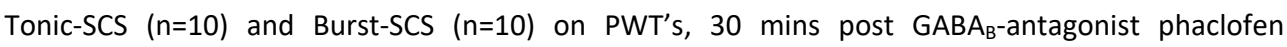
administration. PWT's were assessed at baseline, 15, 30, 45, and 60 mins. of SCS. C) The effect of Tonic-SCS $(n=5)$ and Burst-SCS $(n=5)$ on PWT's, 30 mins post vehicle administration. PWT's were assessed at baseline, 15, 30, 45, and 60 mins. of SCS. D) The effect of Sham-SCS and GABA-antagonists bicuculline $(n=10)$ or phaclofen $(n=10)$ administration on PWT's, 30 mins post antagonist administration. The dotted line represents the average PWT baseline prior to sciatic nerve ligation. (\# $p<0.05$ for Burst-SCS vs. baseline; $* p<0.05$ for Tonic-SCS vs. baseline. 


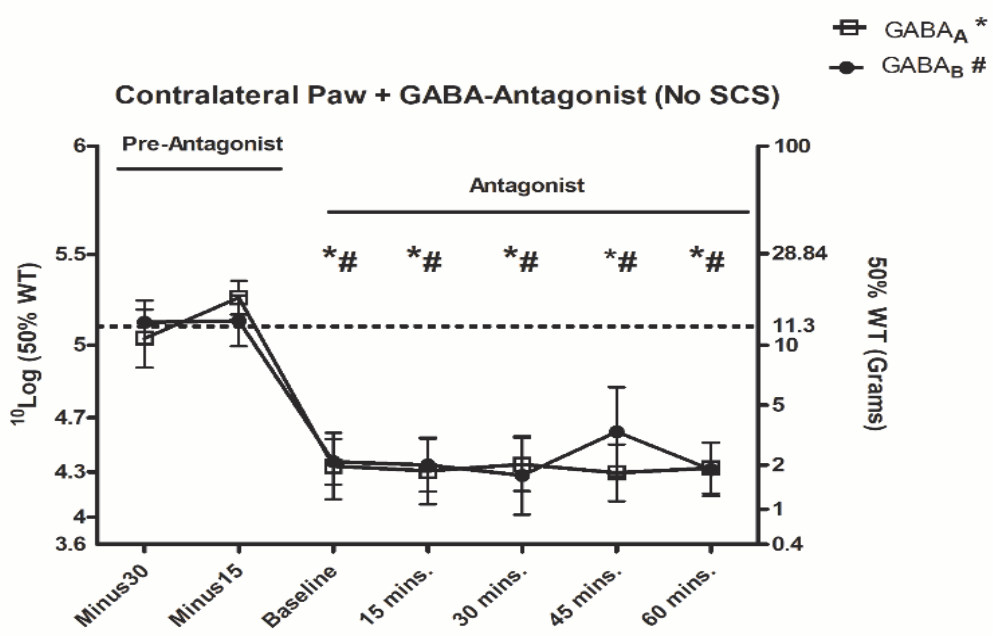

Fig.6: Paw withdrawal thresholds (PWT's) based on sensitivity to von Frey filaments of the contralateral paw 30 mins and 15 mins pre bicuculline $(n=10)$ or phaclofen administration $(n=10)$, and at baseline, 15, 30, 45 and 60 mins post antagonist administration. The dotted line represents the average PWT baseline prior to sciatic nerve ligation. (* $p<0.05$ for bicuculline vs. minus30; $\#<0.05$ for phaclofen vs. minus 30). 


\section{Discussion}

Immunohistochemistry analysis of dorsal horn sections showed a decrease in intracellular GABA levels immediately after Burst SCS. Intracellular GABA levels were significantly decreased compared to Sham-SCS animals and Tonic SCS animals. In a second experiment, administration of a $G A B A_{A}$ - and $G A B A_{B}$-receptor antagonist abolished the analgesic effect of both Tonic SCS and Burst SCS. Based on these findings we conclude that the analgesic effect of Burst-SCS is mediated via a spinal GABAergic mechanism. GABAergic interneurons are known to be important players in the pain gate system that is located in the dorsal horn of the spinal cord $[39,42,43]$, which in its turn is a fundamental component of the working mechanisms underlying Tonic SCS [8-11, 13-17, 44]. Both experimental and clinical studies have documented the importance of segmental GABA-release by turning SCS nonresponders into responders via intrathecal administration of the $G_{A B A_{B}}$ agonist baclofen (in subeffective doses)[16] [17] [18]. The majority of experimental studies on the effect of Tonic SCS were performed in sciatic nerve injury models including the sciatic nerve ligation model (PSNL) and the chronic constriction injury model. [13, 16, 17, 30-33, 37, 38, 45]. Therefore, in order to adequately compare and correlate our findings to previous literature, we deliberately chose to perform our experiments in the partial sciatic nerve ligation model. Initially, we found that intracellular GABA-levels in the rat dorsal horn were significantly decreased following 60 mins of Burst SCS, hence, an indication that Burst SCS does induce the release of GABA in the dorsal horn. Our notion was further confirmed by the results of our second experiment, where we demonstrated that a $G A B A_{A}$-antagonist phaclofen and $G_{B B} A_{B}$-antagonist bicuculline both abolished the pain relieving behavioral effect of Burst and Tonic SCS. Our findings on the effect and mechanism of Tonic SCS are in line with earlier work performed at our laboratory, where it was demonstrated that Tonic SCS decreased intracellular GABA-immunoreactivity in the dorsal horn of PSNL-rats [13]. Perhaps even more important is that, in line with our study, experimental pharmacological work has previously shown that local perfusion with a $\mathrm{GABA}_{B}$-receptor antagonist in the dorsal horn of neuropathic rats transiently abolished the Tonic SCS-induced effect [16]. Meanwhile, preclinical studies conducted also in a sciatic nerve injury model demonstrated that intrathecal administration of the $\mathrm{GABA}_{B}$-agonist baclofen, and the $\mathrm{GABA}_{A}$ agonist muscimol, administered intrathecally, resulted in a marked and long-lasting increase of withdrawal thresholds in rats not receiving adequate pain relief with Tonic SCS (non-responders), although muscimol produced a less prominent threshold increase as compared to baclofen [48]. This and our data shows that Tonic SCS operates by potentiating the spinal GABAergic 


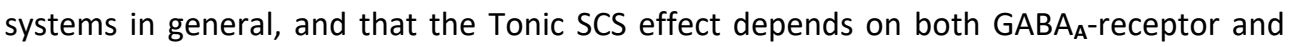
$G_{B B A_{B}}$-receptor signaling, although the SCS-effect seems to be more linked to the $G A B A_{B^{-}}$ receptor system [45]. In our study, administration of a $G_{A B A}$ - and $G_{A B A}-$ receptor antagonist prevented the pain relieving effect of Burst SCS. Our conclusion that the pain relieving effect of Burst-SCS is mediated via spinal GABAergic mechanisms may conflict with findings reported by Crosby and colleagues (2015). Based on electrophysiological analysis of neuronal firing after Burst-SCS, these authors reported that the presence of a GABA $A_{B}$ receptor antagonist did not block the attenuation of dorsal horn neuronal firing, when SCS was applied at $90 \%$ of the Motor Threshold (MT) [28]. Based on these findings the authors conclude that Burst SCS does not act via a spinal GABAergic mechanism whereas Tonic SCS does [31]. However, as the electrophysiological experiments were terminal, no behavioral testing was performed during the conscious state of the animals. Yet, behavioral analysis and thus assessment of the pain relieving effect is known to be the most important indicator of a treatment or compound's translational value [46]. Furthermore, even though the experiments provide novel insights into the working mechanisms underlying Tonic and Burst SCS, the fact that the experiments were performed in a painful cervical nerve root compression rat model makes it difficult to compare the findings to the majority of experimental SCS literature, since most experimental data on pain relief and spinal GABAergic mechanism underlying Tonic and Burst SCS have been documented and studied in peripheral nerve injury models for mononeuropathy [13-17, 29, 32, 33]. It is interesting that in the study by Crosby and colleagues both Burst and Tonic SCS was applied at $90 \%$ MT, an intensity known to induce unwanted side effects in the animals. Our study and analysis was based on previously determined optimal settings for pain relief with Burst SCS (50\% MT) and Tonic SCS (66\% MT) [31, 33]. Moreover, according to the strength-duration curve, SCS administered at 90\%, MT is above perception-threshold, while Burst SCS in the clinical setting is usually applied below perception threshold (paresthesia-free) [24, 25, 47-49]. Experimental evidence and clinical observations indicate that Burst SCS has a delayed washin effect as compared to Tonic SCS [32]. An experimental study by Meuwissen et al. (2018) demonstrated that after 15 minutes Burst SCS does induce pain relief, however, at a suboptimal level, as compared to the plateau-phase which is reached after 30 minutes (when Burst and Tonic SCS reach similar levels of pain relief) [32]. It has been proposed that Burst SCS is capable of modulating both the medial and the lateral pain pathway [25, 27]. As stated by Dr. De Ridder; "the exact mechanism of the selective routing is unknown but could be related to diameter differences at a bifurcation, in which only the burst waveform is powerful enough to overcome the higher transmission resistance of the smaller C-fibers 
that make up the medial pain pathway" [33]. Meanwhile, evidence shows that Tonic SCS, via descending pathways, exerts an inhibitory GABAergic effect in the spinal cord [50-55]. Therefore, it could be hypothesized that 5 minutes of Burst SCS, as applied in the Crosby study, was not sufficient to set in motion the delayed supraspinal mechanisms that lead to optimal pain relief as observed after the effects of Burst SCS have plateaued (30+ minutes) [32]. Additionally, after only 5 minutes of Burst SCS, Crosby and colleagues might have observed a GABA-independent segmental mechanism of Burst SCS, unaffected by the

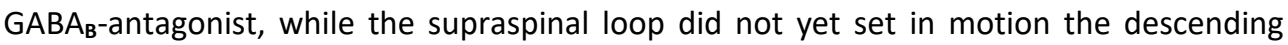
pathways that exert an inhibitory effect via GABAergic mechanisms in the spinal cord. It should be mentioned that in our study we applied biphasic active recharge Burst SCS, while Crosby and colleagues applied passive recharge burst SCS. Whether or not there are physiological or clinical differences between these burst waveforms is unknown [56, 57]. To date, no data from clinical or preclinical studies have been performed that have directly compared passive recharge Burst SCS and active recharge Burst SCS, therefore no conclusive statements regarding the efficacy of these Burst waveforms can be made. However, future research that aims to elucidate the working mechanisms of, and (possible) differences between, these waveforms is desired. Interestingly, our results demonstrate that blocking of the $G_{A B A_{A}}$-receptor does not prevent a significant increase in PWTs after 15 mins of Tonic SCS, while the effect was successfully suppressed during the remaining minutes of stimulation. This suggests that Tonic SCS possesses the ability to, initially, activate GABAindependent pain relieving mechanisms. It is believed that the supraspinal mechanisms of Tonic SCS arise from activation of the 'fast-conducting' lateral pain pathway. Once activated, supraspinal areas activated by the lateral pain pathway may modulate via descending pathways the pain-signals coming in on a segmental level, through, for example, the release of serotonin and norepinephrine at the spinal dorsal horns $[8,11,58]$.

\section{Conclusion}

In conclusion, our data, based on experiments in a validated commonly used experimental chronic neuropathic pain model, demonstrate that spinal GABA plays a role in the working mechanisms of Burst SCS in the attenuation of pain. Further analysis of the GABAergic mechanisms underlying Burst SCS at different time points could shed more light on the exact mechanisms at play. 


\section{References}

1. Kumar, K., S. Rizvi, and S.B. Bnurs, Spinal cord stimulation is effective in management of complex regional pain syndrome I: fact or fiction. Neurosurgery, 2011. 69(3): p. 566-78; discussion 5578-80.

2. Kumar, K., M. Abbas, and S. Rizvi, The use of spinal cord stimulation in pain management. Pain Manag, 2012. 2(2): p. 125-34.

3. Health Quality, O., Spinal cord stimulation for neuropathic pain: an evidence-based analysis. Ont Health Technol Assess Ser, 2005. 5(4): p. 1-78.

4. Taylor, R.S., J.P. Van Buyten, and E. Buchser, Spinal cord stimulation for complex regional pain syndrome: a systematic review of the clinical and cost-effectiveness literature and assessment of prognostic factors. Eur J Pain, 2006. 10(2): p. 91-101.

5. Reig, E. and D. Abejon, Spinal cord stimulation: a 20-year retrospective analysis in 260 patients. Neuromodulation, 2009. 12(3): p. 232-9.

6. Hou, S., K. Kemp, and M. Grabois, A Systematic Evaluation of Burst Spinal Cord Stimulation for Chronic Back and Limb Pain. Neuromodulation, 2016. 19(4): p. 398-405.

7. Linderoth, B. and R.D. Foreman, Conventional and Novel Spinal Stimulation Algorithms: Hypothetical Mechanisms of Action and Comments on Outcomes. Neuromodulation, 2017. 20(6): p. 525-533.

8. Sdrulla, A.D., Y. Guan, and S.N. Raja, Spinal Cord Stimulation: Clinical Efficacy and Potential Mechanisms. Pain Pract, 2018. 18(8): p. 1048-1067.

9. Melzack, R. and P.D. Wall, Pain mechanisms: a new theory. Science, 1965. 150(3699): p. 9719.

10. Guan, Y., Spinal cord stimulation: neurophysiological and neurochemical mechanisms of action. Curr Pain Headache Rep, 2012. 16(3): p. 217-25.

11. Vallejo, R., K. Bradley, and L. Kapural, Spinal Cord Stimulation in Chronic Pain: Mode of Action. Spine (Phila Pa 1976), 2017. 42 Suppl 14: p. S53-S60.

12. Janssen, S.P., et al., Differential GABAergic disinhibition during the development of painful peripheral neuropathy. Neuroscience, 2011. 184: p. 183-94.

13. Janssen, S.P., et al., Decreased intracellular GABA levels contribute to spinal cord stimulationinduced analgesia in rats suffering from painful peripheral neuropathy: the role of KCC2 and $\operatorname{GABA}(A)$ receptor-mediated inhibition. Neurochem Int, 2012. 60(1): p. 21-30.

14. Linderoth, B., et al., Gamma-aminobutyric acid is released in the dorsal horn by electrical spinal cord stimulation: an in vivo microdialysis study in the rat. Neurosurgery, 1994. 34(3): p. 484-8; discussion 488-9.

15. Stiller, C.O., et al., Release of gamma-aminobutyric acid in the dorsal horn and suppression of tactile allodynia by spinal cord stimulation in mononeuropathic rats. Neurosurgery, 1996. 39(2): p. 367-74; discussion 374-5.

16. Cui, J.G., et al., Spinal cord stimulation attenuates augmented dorsal horn release of excitatory amino acids in mononeuropathy via a GABAergic mechanism. Pain, 1997. 73(1): p. 87-95.

17. Cui, J.G., et al., Effect of spinal cord stimulation on tactile hypersensitivity in mononeuropathic rats is potentiated by simultaneous $G A B A(B)$ and adenosine receptor activation. Neurosci Lett, 1998. 247(2-3): p. 183-6.

18. Lind, G., et al., Baclofen-enhanced spinal cord stimulation and intrathecal baclofen alone for neuropathic pain: Long-term outcome of a pilot study. Eur J Pain, 2008. 12(1): p. 132-6.

19. Kriek, N., et al., Preferred frequencies and waveforms for spinal cord stimulation in patients with complex regional pain syndrome: A multicentre, double-blind, randomized and placebocontrolled crossover trial. Eur J Pain, 2016. 
20. Ahmed, S., et al., Burst and high frequency stimulation: underlying mechanism of action. Expert Rev Med Devices, 2018. 15(1): p. 61-70.

21. De Ridder, D., et al., A 2-center comparative study on tonic versus burst spinal cord stimulation: amount of responders and amount of pain suppression. Clin J Pain, 2015. 31(5): p. 433-7.

22. Tiede, J., et al., Novel spinal cord stimulation parameters in patients with predominant back pain. Neuromodulation, 2013. 16(4): p. 370-5.

23. Deer, T., et al., Success Using Neuromodulation With BURST (SUNBURST) Study: Results From a Prospective, Randomized Controlled Trial Using a Novel Burst Waveform. Neuromodulation, 2018. 21(1): p. 56-66.

24. De Ridder, D., et al., Burst spinal cord stimulation: toward paresthesia-free pain suppression. Neurosurgery, 2010. 66(5): p. 986-90.

25. De Ridder, D., et al., Burst spinal cord stimulation for limb and back pain. World Neurosurg, 2013. 80(5): p. 642-649 e1.

26. de Vos, C.C., et al., Burst spinal cord stimulation evaluated in patients with failed back surgery syndrome and painful diabetic neuropathy. Neuromodulation, 2014. 17(2): p. 152-9.

27. De Ridder, D. and S. Vanneste, Burst and Tonic Spinal Cord Stimulation: Different and Common Brain Mechanisms. Neuromodulation, 2016. 19(1): p. 47-59.

28. Crosby, N.D., et al., Burst and Tonic Spinal Cord Stimulation Differentially Activate GABAergic Mechanisms to Attenuate Pain in a Rat Model of Cervical Radiculopathy. IEEE Trans Biomed Eng, 2015. 62(6): p. 1604-13.

29. Seltzer, Z., R. Dubner, and Y. Shir, A novel behavioral model of neuropathic pain disorders produced in rats by partial sciatic nerve injury. Pain, 1990. 43(2): p. 205-18.

30. Truin, M., et al., Successful pain relief in non-responders to spinal cord stimulation: The combined use of ketamine and spinal cord stimulation. European Journal of Pain, 2011. 15(10).

31. Truin, M., et al., Increased efficacy of early spinal cord stimulation in an animal model of neuropathic pain. Eur J Pain, 2011. 15(2): p. 111-7.

32. Meuwissen, K.P.V., et al., Burst Spinal Cord Stimulation in Peripherally Injured Chronic Neuropathic Rats: A Delayed Effect. Pain Pract, 2018.

33. Meuwissen, K.P.V., et al., Conventional-SCS vs. Burst-SCS and the Behavioral Effect on Mechanical Hypersensitivity in a Rat Model of Chronic Neuropathic Pain: Effect of Amplitude. Neuromodulation, 2018. 21(1): p. 19-30.

34. Pluijms, W.A., et al., The effect of spinal cord stimulation frequency in experimental painful diabetic polyneuropathy. Eur J Pain, 2013. 17(9): p. 1338-46.

35. Chaplan, S.R., et al., Quantitative assessment of tactile allodynia in the rat paw. J Neurosci Methods, 1994. 53(1): p. 55-63.

36. Magoul, R., et al., Anatomical distribution and ultrastructural organization of the GABAergic system in the rat spinal cord. An immunocytochemical study using anti-GABA antibodies. Neuroscience, 1987. 20(3): p. 1001-9.

37. Smits, H., et al., Effect of spinal cord stimulation in an animal model of neuropathic pain relates to degree of tactile "allodynia". Neuroscience, 2006. 143(2): p. 541-6.

38. Smits, H., M. van Kleef, and E.A. Joosten, Spinal cord stimulation of dorsal columns in a rat model of neuropathic pain: evidence for a segmental spinal mechanism of pain relief. Pain, 2012. 153(1): p. 177-83.

39. Malan, T.P., H.P. Mata, and F. Porreca, Spinal GABA(A) and $G A B A(B)$ receptor pharmacology in a rat model of neuropathic pain. Anesthesiology, 2002. 96(5): p. 1161-7.

40. van Beek, M., et al., Spinal cord stimulation in experimental chronic painful diabetic polyneuropathy: Delayed effect of High-frequency stimulation. Eur J Pain, 2016.

41. Mills, C., et al., Estimating efficacy and drug ED50's using von Frey thresholds: impact of weber's law and log transformation. J Pain, 2012. 13(6): p. 519-23. 
42. Hao, J.X., X.J. Xu, and Z. Wiesenfeld-Hallin, Intrathecal gamma-aminobutyric acidB (GABAB) receptor antagonist CGP 35348 induces hypersensitivity to mechanical stimuli in the rat. Neurosci Lett, 1994. 182(2): p. 299-302.

43. Sivilotti, L. and C.J. Woolf, The contribution of GABAA and glycine receptors to central sensitization: disinhibition and touch-evoked allodynia in the spinal cord. J Neurophysiol, 1994. 72(1): p. 169-79.

44. Smits, H., et al., Experimental spinal cord stimulation and neuropathic pain: mechanism of action, technical aspects, and effectiveness. Pain Pract, 2013. 13(2): p. 154-68.

45. Cui, J.G., B. Linderoth, and B.A. Meyerson, Effects of spinal cord stimulation on touch-evoked allodynia involve GABAergic mechanisms. An experimental study in the mononeuropathic rat. Pain, 1996. 66(2-3): p. 287-95.

46. Mogil, J.S., Animal models of pain: progress and challenges. Nat Rev Neurosci, 2009. 10(4): p. 283-94.

47. Jonathon Miller, S.E., Eric Buchser, Lisa M. Johanek, Yun Guan, MD, Bengt Linderoth., Parameters of Spinal Cord Stimulation and Their Role in Electrical Charge Delivery. Neuromodulation: Technology at the Neural Interface, 2016: p. 12.

48. Abejon, D., et al., Is the introduction of another variable to the strength-duration curve necessary in neurostimulation? Neuromodulation, 2015. 18(3): p. 182-90; discussion 190.

49. Holsheimer, J., et al., The effect of pulse width and contact configuration on paresthesia coverage in spinal cord stimulation. Neurosurgery, 2011. 68(5): p. 1452-61; discussion 1461.

50. Barchini, J., et al., Spinal segmental and supraspinal mechanisms underlying the pain-relieving effects of spinal cord stimulation: an experimental study in a rat model of neuropathy. Neuroscience, 2012. 215: p. 196-208.

51. Song, Z., et al., Pain relief by spinal cord stimulation involves serotonergic mechanisms: an experimental study in a rat model of mononeuropathy. Pain, 2009. 147(1-3): p. 241-8.

52. Linderoth, B., et al., Dorsal column stimulation induces release of serotonin and substance $P$ in the cat dorsal horn. Neurosurgery, 1992. 31(2): p. 289-96; discussion 296-7.

53. Saade, N.E. and S.J. Jabbur, Nociceptive behavior in animal models for peripheral neuropathy: spinal and supraspinal mechanisms. Prog Neurobiol, 2008. 86(1): p. 22-47.

54. Foreman, R.D. and B. Linderoth, Neural mechanisms of spinal cord stimulation. Int Rev Neurobiol, 2012. 107: p. 87-119.

55. Linderoth, B. and R.D. Foreman, Physiology of spinal cord stimulation: review and update. Neuromodulation, 1999. 2(3): p. 150-64.

56. Meuwissen, K.P.V., et al., Response to: Fundamental Differences in Burst Stimulation Waveform Design: Eliminating Confusion in the Marketplace. Neuromodulation, 2018. 21(7): p. 721-722.

57. Falowski, S.M., Fundamental Differences in Burst Stimulation Waveform Design: Eliminating Confusion in the Marketplace. Neuromodulation, 2018. 21(3): p. 320.

58. Saade, N.E., et al., Modulation of segmental mechanisms by activation of a dorsal column brainstem spinal loop. Brain Res, 1984. 310(1): p. 180-4. 



\section{CHAPTER 5}

\section{Burst and Tonic Spinal Cord Stimulation in the Mechanical Conflict-Avoidance System: Cognitive-Motivational Aspects}

Koen P.V. Meuwissen, Maarten van Beek,, Elbert A. J. Joosten.

Accepted in Journal of Neuromodulation: Technology at the Neural Interface 2019

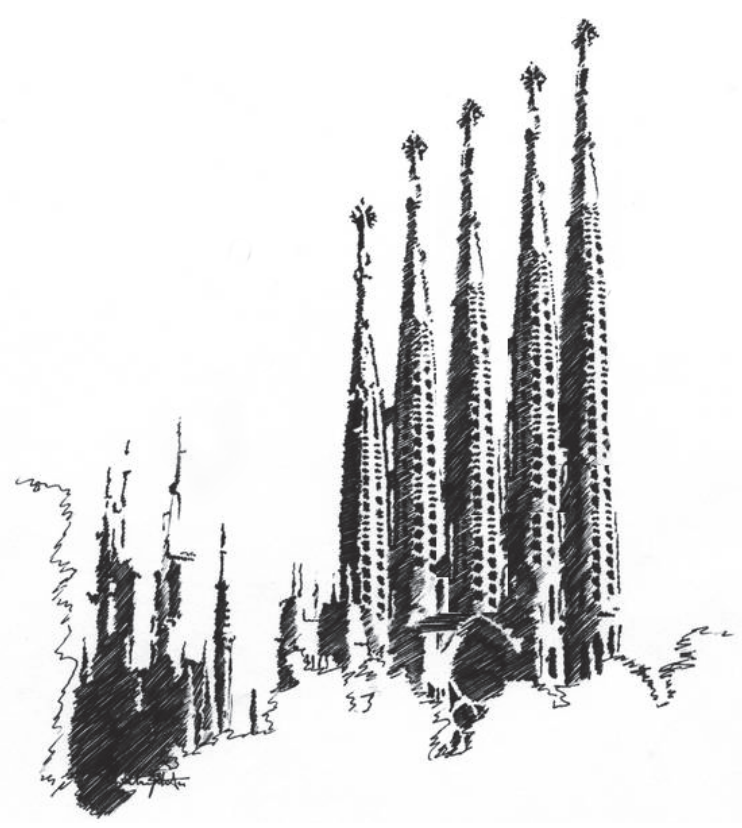





\section{Abstract}

Background: Clinical research suggests that a novel spinal cord stimulation (SCS) waveform, known as Burst-SCS, specifically targets cognitive-motivational aspects of pain. The objective of the present study was to assess the cognitive-motivational aspects of Tonicand Burst SCS-induced pain relief, by means of exit latency in the Mechanical ConflictAvoidance System (MCAS), in a rat model of chronic neuropathic pain.

Methods: Exit latency on the MCAS operant testing setup was evaluated at various probe heights for rats $(n=26)$ with chronic neuropathic pain induced by a partial sciatic nerve ligation (PSNL). Von Frey paw withdrawal analysis was performed to assess mechanical hypersensitivity. In a second experiment $(n=12)$, the behavioral effect of Tonic SCS or biphasic Burst SCS on both Von Frey analysis and MCAS exit latency was assessed.

Results: Burst SCS exit latencies differed significantly from Tonic SCS exit latencies at $4 \mathrm{~mm}$ probe height ( 3.8 vs 5.8 secs respectively; $p<0.01$ ) and $5 \mathrm{~mm}$ probe height ( 3.2 vs 5.4 secs respectively; $p<0.05)$. This difference was not detected with reflex-based Von Frey testing (Tonic-SCS vs. Burst-SCS at 30 mins stimulation: $p=0.73$, and at 60 mins stimulation; $p=$ $0.42)$

Conclusions: Testing of MCAS exit latency allows for detection of cognitive-motivational pain relieving aspects induced by either Tonic- or Burst-SCS in treatment of chronic neuropathic rats. Our behavioral findings strongly suggest that Burst-SCS specifically affects, much more than Tonic-SCS, the processing of cognitive-motivational aspects of pain. 


\section{Introduction}

The preclinical Spinal Cord Stimulation (SCS) field calls for an operant testing method able to assess cognitive-motivational aspects of pain [1]. This is becoming increasingly important now that recent electroencephalography findings suggest that Burst and Tonic SCS may have different supraspinal working mechanisms; Burst SCS is hypothesized to selectively modulate brain areas associated with the processing of attention-related cognitivemotivational aspects of pain $[2,3]$. Meanwhile, the majority of preclinical SCS studies still rely on reflex-mediated Von Frey analysis, a technique unable to assess supraspinal cognitive-motivational aspects of pain [4-12]. Recently, an operant testing method was introduced which assesses cognitive and motivational aspects of pain in animals: the Mechanical Conflict-Avoidance System (MCAS) [13]. With the MCAS the animal is placed in a brightly lit compartment which leads to a passage with a height-adjustable array of nociceptive probes. The animal needs to cross the nociceptive probes to enter the innately preferred dark area. The "lesser of two evils principle" forces the animal to choose between two opposing motivational drives: to stay in the aversive, yet non-noxious, brightly lit compartment, or, to cross the noxious probes, which is rewarded by the innately preferred dark compartment. In order to resolve this conflict it is hypothesized that the animal applies a "cost-benefit" analysis including the level of ongoing pain, the height of the probes, and the averseness of the light (= negative reinforcement) [14]. In general, as ongoing pain intensity and/ or probe height increases, animals require more time to exit the light chamber. Latency to exit the light chamber (defined as time from light being turned on to having all four paws on the probe bed) has been shown to be a stimulus-dependent measure in the Coy-MCAS system. Chronic neuropathic pain, induced by chronic constriction of the sciatic nerve, has been shown to affect latency to exit the bright compartment in the MCAS [15]. However, other neuropathic pain models still remain to be validated in the MCAS-setup. Furthermore, it is of great interest to the preclinical SCS field to assess whether the MCAS can shed light on the supraspinal mechanisms of Burst and Tonic SCS by addressing the cognitive-motivational aspects of pain that are becoming increasingly important for the assessment of novel SCS waveforms [3, 16].

Our first objective was to assess the effect of the partial sciatic nerve ligation rat model for chronic neuropathic pain on exit latency in the MCAS. As cognitive-motivational aspects cannot be detected by reflex-based Von Frey analysis, our second objective was to assess the cognitive-motivational aspects of Tonic- and Burst SCS-induced pain relief, by means of exit latency in the MCAS operant testing system. We hypothesized that both Tonic- and 
Burst SCS would reduce MCAS exit latency, which will suggest a role for cognitivemotivational aspects in SCS-induced pain relief. Furthermore, based on literature indicating that Burst SCS selectively modulates brain areas associated with the processing of attentionrelated cognitive-motivational aspects of pain, we hypothesized that Burst-SCS would have a stronger effect on MCAS exit latency, as compared to Tonic-SCS [2, 3]. 


\section{Materials and Methods}

\section{Ethics Statement}

All experiments were performed in accordance with the European Directive for the Protection of Vertebrate Animals Used for Experimental and Other Scientific Purposes (86/609/EU). The protocol was approved by the Animal Research Committee of the Maastricht University Medical Centre (DEC-protocol 2014-086).

\section{Animals}

All experiments were performed using male Sprague Dawley rats $(n=38)$, which were youngadult (5 weeks of age) at the start of the experiment (150-200 g). Animals were housed in groups of 2 , in filter-top polycarbonate cages in a climate-controlled vivarium maintained under controlled temperature $\left(21 \pm 1^{\circ} \mathrm{C}\right)$, relative humidity $(55 \pm 15 \%)$ and artificial lighting (12:12 hour light/dark cycle) with distilled water and rodent chow available ad libitum. The vivarium was equipped with a mobile radio, continuously producing background music at 45 decibels, in order to desensitize the animals for translocation and experimenter-related noise. All procedures were conducted between 09:00 and 16:00 hours.

\section{Partial Sciatic Nerve Ligation (PSNL)}

A unilateral ligation of the left sciatic nerve was performed as described by Seltzer et al. 1990 [17], and previously applied in our laboratory [9, 10]. In short, animals were anesthetized with 3-5\% isoflurane (Abbott Laboratories Ltd., Kent, U.K.). The left sciatic nerve was exposed by blunt dissection and carefully freed from surrounding connective tissue. For sham-PSNL animals, the sciatic nerve was left unaffected and the wound was closed with a $4 / 0$ silk suture. For PSNL animals, distal to the posterior biceps semitendinosus, but proximal to the little fat pad that lies a few millimeters distal to this site, the sciatic nerve was partially ligated. An $8 / 0$ non-absorbable silk suture was used to ligate approximately $1 / 3$ of the diameter of the left sciatic nerve. After ligation, the wound was closed with a $4 / 0$ silk suture. The presence of mechanical hypersensitivity was considered successful if at 13 days post-surgery, paw withdrawal thresholds (PWTs) to Von Frey stimuli $\left({ }^{10} \log (50 \%)\right)$ were decreased by 0.2 units compared with baseline (day 0$)[9$, 10]. 


\section{Assessment of mechanical hypersensitivity (von Frey assay)}

Assessment of PWTs was performed according to the standard protocol [7-10]. Mechanical hypersensitivity based on PWT was assessed according to the 'up-down method' [18]. The $50 \%$ PWT was calculated after completion of a sequence of six consecutive responses. A cut-off value of $28.84 \mathrm{~g}$ was defined. For statistical analysis, 50\% PWTs were logarithmically transformed to obtain a linear scale.

\section{Mechanical Conflict-Avoidance System (MCAS)}

Familiarization and Training. Familiarization and Training was conducted as described in detail by Harte et al. 2016 [13].

Testing Procedure. Rats underwent room acclimation for 30 minutes prior to the start of behavioral testing each day. Rats were placed individually, in random order, into the startcompartment with the light turned off and the exit door closed. Animals were acclimatized to the dark for 15 seconds, before the compartment light was turned on for the duration of the test. Twenty seconds after the light was turned on the exit door was opened. The latency to exit the light compartment was recorded by means of a stopwatch, starting from the time the exit door was opened until all four paws were placed upon the nociceptive probes. If the animal reached the dark compartment, the door was closed and the rat was returned gently to its home cage after being rewarded with 20 seconds of darkness. Failure to exit the light compartment within 20 seconds after opening of the exit door was marked as 'failed exit', which resulted in the exit door being closed and the rat being returned to its home cage. Rats that successfully escaped the light compartment but failed to enter the dark compartment after 120 seconds, were marked as failed cross, and were returned to their home cage until the next trial. The test procedure was repeated three times (trials) per test session (a minimum of 20 minutes between trials), with one test session per probe height, per day. It was decided to introduce the different probe heights in a non-randomized ascending order over the six test days (starting with $0.5 \mathrm{~mm}$ on test day 1 , followed by 1 $\mathrm{mm}$ on test day 2, $2 \mathrm{~mm}$ on test day $3,3 \mathrm{~mm}$ on test day $4,4 \mathrm{~mm}$ on test day 5 , and $5 \mathrm{~mm}$ on day 6). All test sessions were video-recorded with an ultra-wide angle glass lens camera. Recordings were started immediately after the animal was placed inside the startcompartment (with the light turned off), and were continued until the animal was returned to its home cage. After finalization of the entire experiment all recordings of exit latency were re-timed with a stopwatch, and compared with the manually collected data acquired during the experiment. 


\section{Implantation of spinal cord stimulation device}

The implantation of the SCS device was performed according to the standard protocol $[4,6$, $7,10-12,19]$. In short, the spinal cord was exposed by a midline, lumbar incision, followed by laminectomy at level T13. During the full procedure, the dura was kept intact. A custommade cylindrical 4-contact lead $(0.72 \mathrm{~mm}$ diameter; Boston Scientific Neuromodulation, Valencia, CA, USA) was introduced into the epidural space as previously was performed in Meuwissen et al. [6, 7]. The electrode was located caudally below the adjacent one or two lamina. Electrode configuration was set at alternating cathode and anode settings (rostral to caudal: +-+-$)$. After implantation of the electrodes, the rats were given 2 days for recovery prior to the initiation of SCS.

\section{Spinal cord stimulation (SCS)}

Tonic-SCS was performed according to the protocol described in Meuwissen et al. [6, 7]. The stimulator was set to deliver constant current biphasic stimulation, with a frequency of $50 \mathrm{~Hz}$ and a pulse width of $200 \mu \mathrm{S}$ at $66 \%$ of the Motor Threshold for Tonic SCS ( $\mathrm{n}=5$ ) [7, 10]. For biphasic Burst SCS $(n=5)$ the stimulator was set to an interburst-frequency $40 \mathrm{~Hz}$, a pulse width $1000 \mu \mathrm{S}$, and 5 active biphasic spikes at $449 \mathrm{~Hz}$ intraburst frequency at $50 \%$ of the Motor Threshold [6, 7]. Animals were stimulated for $30 \mathrm{~min}$. in the MCAS-set-up, with the light turned off and the door closed. Subsequently stimulation was continued during the MCAS-testing session, which was performed according to the standard MCAS-testing protocol. The animals were randomized across experimental groups, and the investigator was blinded to the experimental condition during behavioral testing. SCS in the MCASsystem was performed with use of a custom-made experimental apparatus. The cables from the stimulator were guided to a swivel, which allowed $360^{\circ}$ free movement. The cable was then further guided to the SCS connectors in the neck of the animal by means of a fendertension spring system which generated the appropriate amount of tension in order to prevent any slacking of the cables. The upper cover of the MCAS crossing area was removed to create access for the SCS-system. Removal of the upper cover was performed at the start of the experiment, before the training phase, to prevent distraction of the animal due to removal. Furthermore, animals underwent an additional training period of three days with the complete experimental apparatus before start of the SCS experiment, for the animals to become familiarized with the experimental apparatus before testing. To control for the effects of SCS in the MCAS-system, a sham-SCS group was included $(n=2)$. 


\section{Timeline of experiments}

After acclimatization to the vivarium, a two-day familiarization period was initiated, followed by a five-day training period [13]. This was followed by a two-day rest period, after which the baseline (pre-PSNL) test period was initiated, which consisted of six subsequent days of testing as described above. Subsequently, animals received either PSNL $(n=18)$ or sham-PSNL surgery $(n=8)$. Following a 14 day observation period, during which animals underwent von Frey behavioral analysis in order to assess whether mechanical hypersensitivity was successfully induced, animals were subjected to a two-day "refresher" training period (PSNL [ $n=17]$ and sham-PSNL [ $n=8]$ ). During these two days it was noted whether animals still displayed stable exit behavior. Finally, a six day, post-PSNL, testing period was initiated, identical to the baseline test period (PSNL $[n=17]$ and sham-PSNL [( $n$ = 8]) (Fig.1). In a second experiment (Fig.2), 12 animals received PSNL surgery and were subjected to the training- and testing period as described above. This period was followed by the implantation of the SCS-electrode, and a post-implantation (Pre-SCS) test week to assess possible implantation-related effects on the MCAS-outcome. Subsequently, two animals received Sham SCS, and the other animals $(n=10)$ were placed in the MCAS-system after which simultaneous MCAS-SCS testing was performed as described in section 2.7. Five animals received Burst SCS $(n=5)$, while the other five animals received Tonic SCS $(n=5)$.

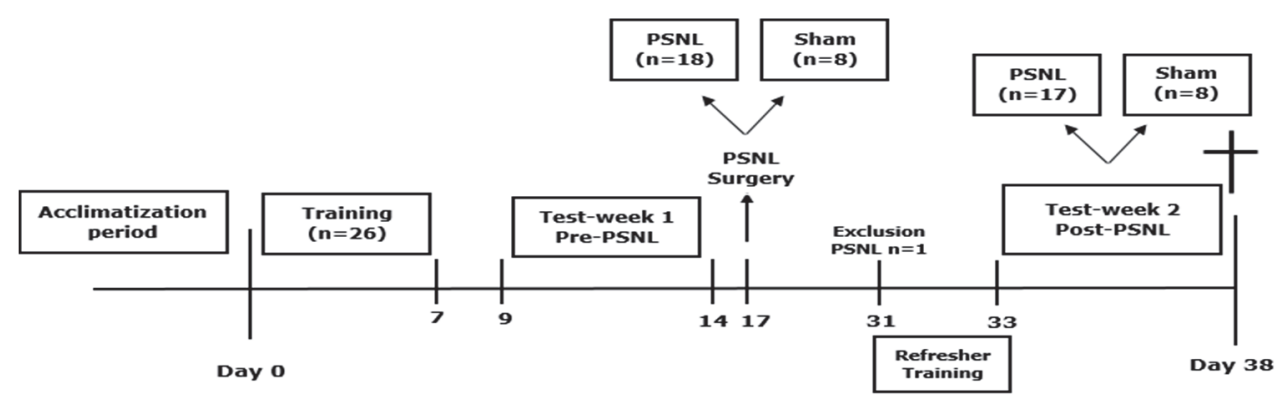

Fig.1: Timeline experiment 1 


\section{Data Analysis}

The PWTs to von Frey filaments are presented as mean \pm standard error of the mean (SEM). For statistical analysis von Frey data were logarithmically transformed to obtain a linear scale to account for Weber's Law [20]. For the analysis of differences in the withdrawal thresholds between groups, ipsilateral and contralateral PWTs were compared using paired-sampled T-tests. To account for skewness of data at higher probe heights MCAS exit latencies were logarithmically transformed. Effects of probe height on exit latencies were analyzed using repeated-measures Analysis Of Variance (ANOVA). Probe height (6 levels: 0 , $1,2,3,4$, and $5 \mathrm{~mm}$ ) was assigned as within-subjects factor and the experimental group (Sham-PSNLvs PSNL or Pre-SCS vs SCS) was assigned as between-subjects factor. If the assumption of sphericity was violated, the Greenhouse-Geisser correction was used to correct the degrees of freedom in subsequent univariate analyses. Multivariate analyses were used to test for differences between groups at specific probe heights. To assess withingroup differences for the Sham-PSNL vs PSNL group, pre- vs post-surgery, and the Pre-SCS vs SCS group, paired-samples T-tests were performed. Furthermore, bivariate correlation between bodyweights, von Frey data, and MCAS exit- latencies were performed to identify possible causalities between the different outcome measures. All statistical analyses were performed with $\alpha=0.05$ using IBM SPSS statistics 23 .

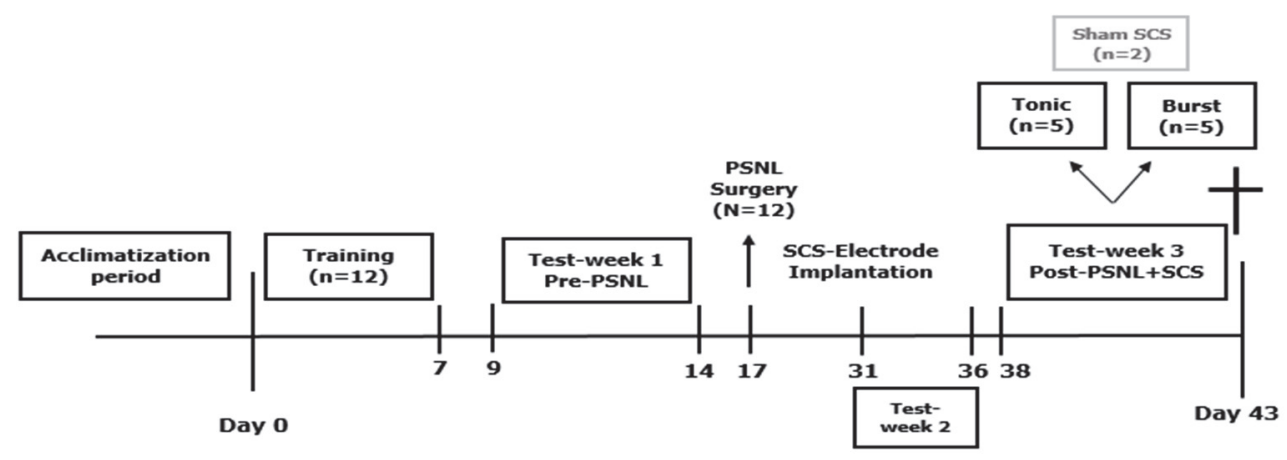

Fig.2: Timeline experiment 2 


\section{Results}

\section{Development of mechanical hypersensitivity (von Frey) in chronic neuropathic rats}

Pre-nerve injury, PWTs of the ipsilateral hind paws were comparable with the PWTs of the contralateral hind paws (ipsilateral $11.6 \pm 1.5 \mathrm{~g}$ vs. contralateral $11.1 \pm 1.3 \mathrm{~g}$ ) in all animals (at day 16 ) in experiment 1 (Fig.3A), and in experiment 2 (ipsilateral $10.9 \pm 1.1 \mathrm{~g}$ vs. contralateral $11.4 \pm 1.5 \mathrm{~g}$ ) (see Fig.3B). Out of the 18 animals that received a unilateral PSNL in experiment 1 , one animal did not develop mechanical hypersensitivity, and was excluded from the study (pre- and post-surgery). The remaining 17 animals qualified as hypersensitive to mechanical stimulation by von Frey filaments (ipsilateral average PWTs: $11.6 \pm 1.5 \mathrm{~g}$ [pre-lesion] vs. $1.6 \pm 0.9 \mathrm{~g}$ [post-lesion]; $p=.0057$ ) (see Methods) and were selected for the PSNL-group (Fig. 3A). The ipsilateral hindpaw PWTs of the PSNL-group were significantly lower than the ipsilateral hindpaw PWTs of the sham-operated animals $(p=$ 0.012). In the Sham-PSNL group, no significant difference between ipsilateral and contralateral PWTs was observed $(p=0.27)$, following the Sham-PSNL surgery, therefore, all 8 animals were selected for the Sham-PSNL group. Out of the 12 PSNL-animals in experiment 2 , all 12 animals were qualified as hypersensitive due to increased response to mechanical stimulation by von Frey filaments (ipsilateral average PWTs: $10.9 \pm 1.1 \mathrm{~g}$ (prelesion) vs. $1.3 \pm 1.6 \mathrm{~g}$ (post-lesion); $p=0.0042$ ) (see Methods) and were selected for the SCSgroup (Tonic or Burst SCS) (Fig.3B).

\section{Development of Tactile Hypersensitivity}
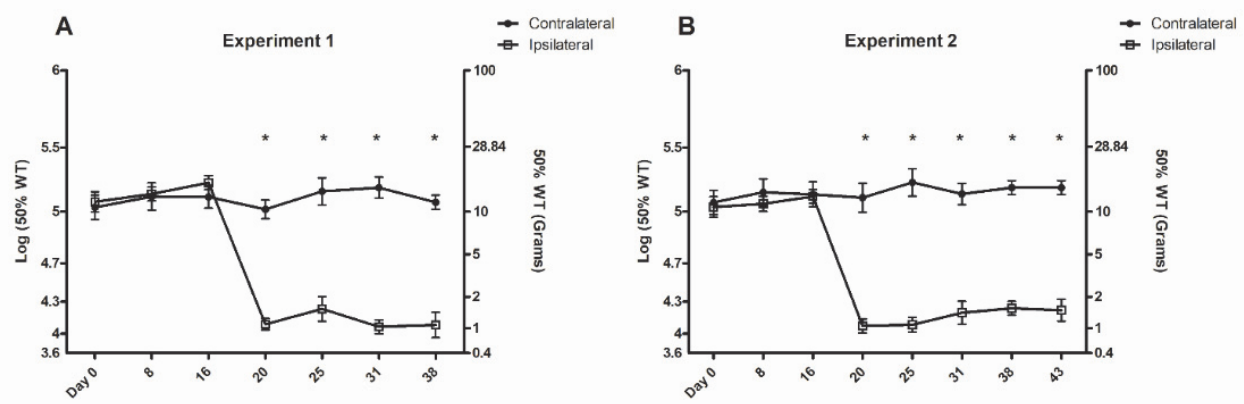

Fig. 3 A: The effect of a Partial Sciatic Nerve Ligation (PSNL) $(n=17)$ on PWTs in Experiment 1. After PSNL, the ipsilateral PWTs of the PSNL-group were significantly decreased at day 20, 25, 31 and 38, compared to the contralateral PWTs. B: The effect of a PSNL $(n=12)$ on PWTs in Experiment 2. After PSNL, the ipsilateral PWTs were significantly decreased at day 20, 25, 31, 38 and 43 compared to contralateral PWTs. 
Chapter 5

\section{Effect of SCS: von Frey analysis}

In animals of the SCS-group paw withdrawal thresholds (PWTs) to von Frey filaments was assessed before the start of SCS treatment (Tonic or Burst SCS) and at 30 and 60 mins after stimulation was turned on. No significant differences were observed in ipsilateral paw PWTs at baseline, pre-SCS, between groups ( $p=0.54$; Tonic-SCS $1.2 \pm 0.5 \mathrm{~g}[\mathrm{n}=5]$ vs. Burst-SCS 1.3 $\pm 0.4 \mathrm{~g}[\mathrm{n}=5])$. After 30 mins of stimulation, PWTs of both the Burst-SCS group $(p=0.0021$; $1.3 \pm 0.4$ g vs. $9.1 \pm 1.1 \mathrm{~g}$ ) and Tonic-SCS group ( $p=0.028 ; 1.2 \pm 0.5 \mathrm{~g}$ vs. $9.5 \pm 1.5 \mathrm{~g}$ ) significantly differed from baseline PWTs (Fig.5). After 60 mins of stimulation, PWTs of both the Burst-SCS group ( $p=0.0016 ; 1.3 \pm 0.4 \mathrm{~g}$ vs. $10.4 \pm 1.3 \mathrm{~g}$ ) and Tonic-SCS group ( $p=0.034$; $1.2 \pm 0.5 \mathrm{~g}$ vs.9.1 $\pm 1.4 \mathrm{~g}$ ) significantly differed from baseline PWTs (Fig. 5). PWTs of the Burst-SCS group and Tonic-SCS group did not significantly differ at 30 mins of stimulation ( $p$ $=0.73$; Tonic-SCS $9.5 \pm 1.5 \mathrm{~g}[\mathrm{n}=5]$ vs. Burst-SCS $9.1 \pm 1.1 \mathrm{~g}[\mathrm{n}=5])$ and 60 mins of stimulation ( $p=0.42$; Tonic-SCS $9.1 \pm 1.4 \mathrm{~g}[\mathrm{n}=5$ ] vs. Burst-SCS $10.4 \pm 1.3 \mathrm{~g}[\mathrm{n}=5]$ ) (Fig.5).

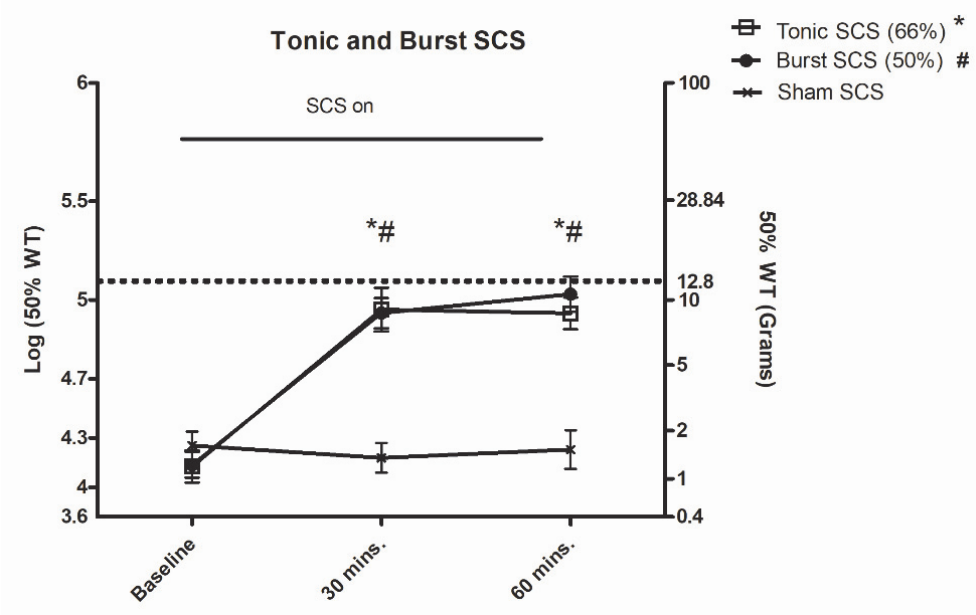

Fig. 5: The effect of Tonic-SCS ( $n=5)$ and Burst-SCS $(n=5)$ on PWTs based on sensitivity to von Frey filaments. PWTs were assessed at baseline (stimulation off) and 30 and 60 mins of SCS. The dotted line represents the average PWT baseline prior to sciatic nerve ligation ( ${ }^{*} p<0.05$ for Tonic SCS compared with pre-SCS baseline PWTs, \# $p<0.05$ for Burst SCS compared with pre-SCS baseline PWTs). 


\section{Mechanical Conflict-Avoidance Test in chronic neuropathic rats}

\section{Exit Latency}

Stimulus-response functions were obtained for PSNL $(n=17)$ and sham-PSNL rats $(n=8)$ in the MCAS both at pre-surgery (days 9-14) and at post-surgery (days 33-38). Pre-surgery: a significant main effect of probe height $(\mathrm{F} 3.264=21.971 ; p<0.001)$, but no significant interaction between probe height and group was found, suggesting that both PSNL and Sham-PSNL animals exhibited equal increase in exit latency as a function of probe height. Post-surgery: a significant main effect of probe height was noted (F3.089 $=19.722 ; p<$ 0.001 ) (Fig 4). No significant interaction effect between probe height and group was observed. Tests of Between-subject effects approached significance ( $F 1=3.427 ; p=0.077$ ), suggesting that exit latencies were different for PSNL- and sham-PSNL animals, postsurgery. Furthermore, at $4 \mathrm{~mm}$ probe height, the exit latencies of the PSNL group and shamPSNL group significantly differed (18.84 vs $10.62 \mathrm{~s} ; p=.038$ ), whereas exit latencies of the PSNL group and sham-PSNL group at $3 \mathrm{~mm}$ probe height closely approached significance (9.64 vs $5.5 \mathrm{~s} ; p=0.053)$.

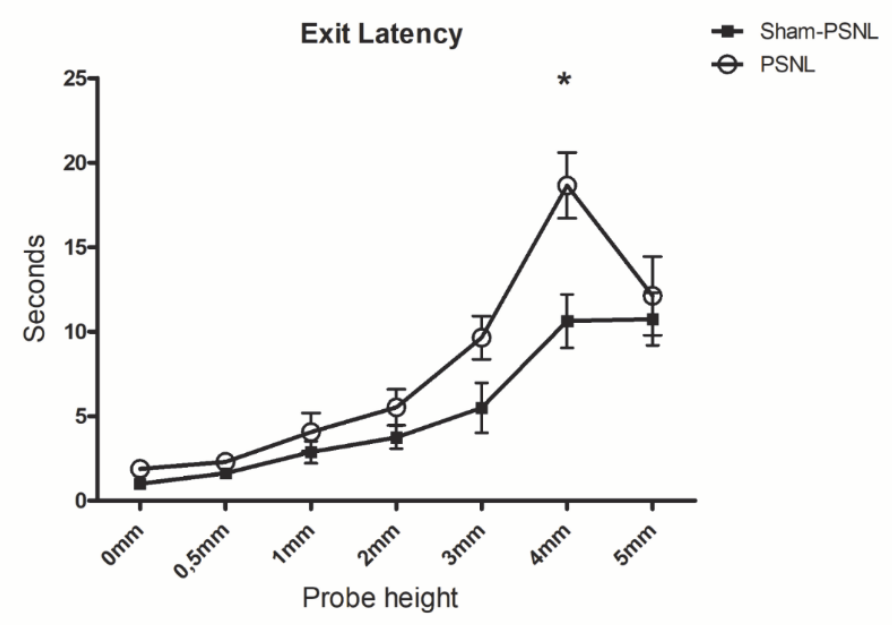

Fig. 4: Stimulus response relationship of escape latency as a function of probe height between, PSNLrats $(n=17)$, and sham-PSNL rats $(n=8)$. As probe height increases, escape latency also increases in both experimental groups $(p<0.001)$. Escape latencies of PSNL rats were significantly greater than those of sham-PSNL rats at $4 \mathrm{~mm}$ probe height (18.84 vs $10.62 \mathrm{~s} ; p=0.038$ ). 


\section{Effect of SCS: MCAS exit latency}

Post-PSNL and Pre-SCS MCAS exit latencies did not significantly differ (F1 $=0.2717 ; p=$ .6069). Thus, the implantation of the electrode itself did not have a significant effect on MCAS exit latency. Group differences in MCAS exit latency were assessed for the Pre-Tonic SCS group (the Tonic SCS group pre-stimulation) and Tonic SCS group ( $n=5)$; a significant main effect of probe height ( $\mathrm{F} 5=21.92 ; p<0.0001)$, and a significant interaction between probe height and group (F5 $=3.255 ; p=0.0147$ ) was noted (Fig 6), which indicates that MCAS exit latencies were different pre-Tonic SCS and during Tonic SCS. This was confirmed by a significant between subjects effect, where the pre-Tonic SCS group and Tonic SCS group significantly differed overall in exit latencies ( $F 1=22.42 ; p=.0015)$. Furthermore, Pre-Tonic SCS and Tonic SCS groups significantly differed at $2 \mathrm{~mm}$ probe height (6.2 vs 3.0 secs; $p<$ .05 ), $3 \mathrm{~mm}$ probe height ( $7.8 \mathrm{vs} 3.4$ secs; $p<.001$ ), and $4 \mathrm{~mm}$ probe height (10.0 vs $5.8 \mathrm{~s} ; p$ $<.01)$.

Group differences in MCAS exit latency were also assessed for the pre-Burst SCS (the Burst SCS group pre-stimulation) and Burst SCS group $(n=5)$; a significant main effect of probe height ( $\mathrm{F} 5=13.78 ; p<0.0001$ ), and a significant interaction between probe height and group (F5 $=7.797 ; p=0.0001$ ) was noted (Fig 7), which indicates that MCAS exit latencies differed between pre-Burst SCS and during Burst SCS. This was confirmed by a significant between subjects effect, where the pre-Burst SCS group and Burst SCS group significantly differed overall in exit latencies ( $F 1=31.49 ; p=0.0005)$. Furthermore, preBurst SCS and Burst SCS groups significantly differed at $2 \mathrm{~mm}$ probe height (7.2 vs 2.4 secs; $p<0.01$ ), $3 \mathrm{~mm}$ probe height ( 9.0 vs 2.8 secs; $p<0.001$ ), $4 \mathrm{~mm}$ probe height ( 9.6 vs $3.8 ; p<$ 0.001 ), and $5 \mathrm{~mm}$ probe height (10.8 vs 3.2 secs; $p<0.001$ ). 


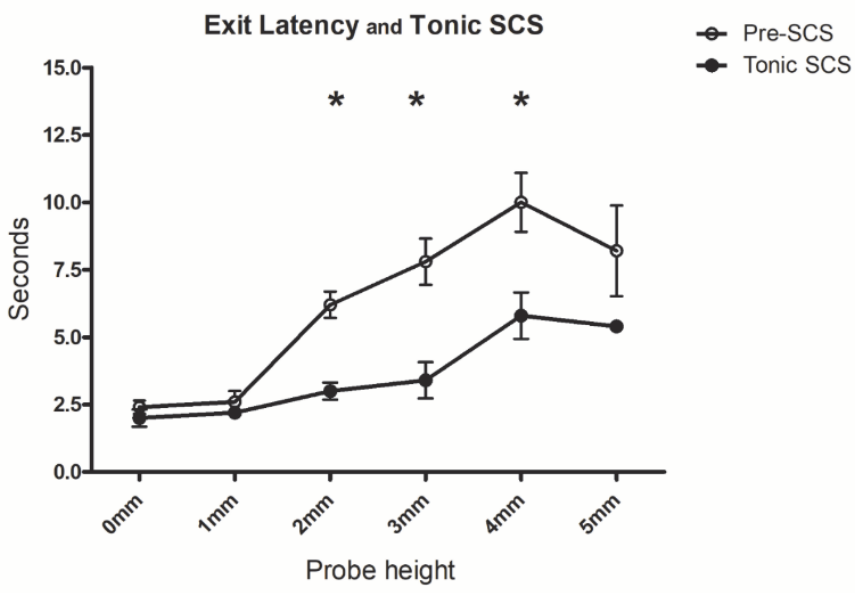

Fig. 6: Stimulus response relationship of escape latency as a function of probe height between PreSCS group and Tonic SCS group $(n=5)$. Escape latencies of SCS rats were significantly lower than those of Pre-SCS group rats at $2 \mathrm{~mm}$ probe height (6.2 vs 3.0 secs; $p<0.05$ ), $3 \mathrm{~mm}$ probeheight (7.8 vs 3.4 secs; $p<0.001), 4 \mathrm{~mm}$ probe height (10.0 vs 5.8 secs; $p<0.01)$, and $5 \mathrm{~mm}$ probeheight (8.2 vs 5.0 secs; $p<0.05)$.

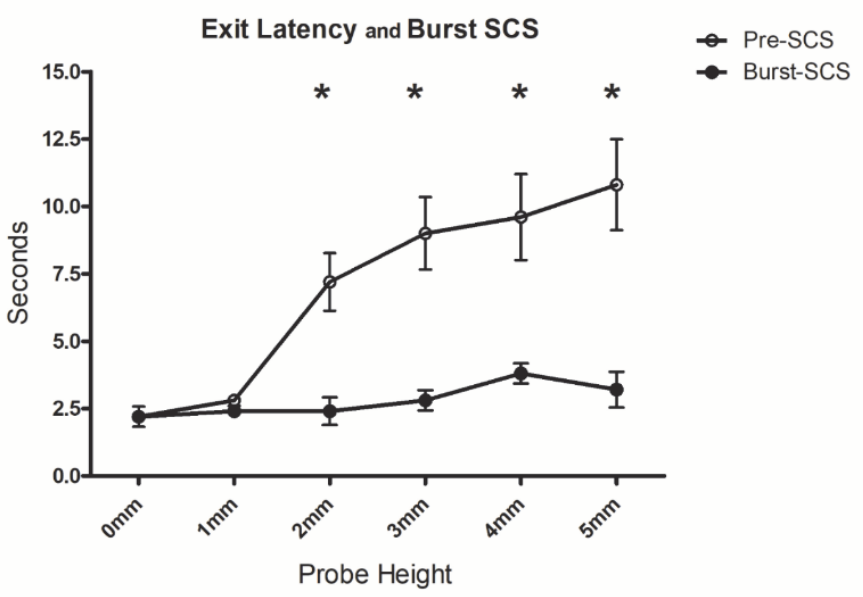

Fig. 7: Stimulus response relationship of escape latency as a function of probe height between PreSCS group and Burst SCS group $(n=5)$. Escape latencies of SCS rats were significantly lower than those of Pre-SCS group rats at $2 \mathrm{~mm}$ probe height (7.2 vs 2.4 secs; $p<0.01$ ), $3 \mathrm{~mm}$ probe height (9.0 vs 2.8 secs; $p<.001)$, $4 \mathrm{~mm}$ probe height (9.6 vs 3.8 secs; $p<0.001)$, and $5 \mathrm{~mm}$ probe height (10.8 vs 3.2 secs; $p<0.001)$. 
Area Between the Curve $(A B C)$ analysis revealed a significant difference between the $A B C$ for Pre-Burst and Burst and the ABC for Pre-Tonic and Tonic SCS (24.6 vs 15.4 secs respectively; $p=0.0225)$. Furthermore, post-hoc analysis revealed a significant difference between the Tonic-SCS group and the Burst-SCS group at $4 \mathrm{~mm}$ probe height (5.8 vs 3.8 secs respectively; $p<.01$ ) and $5 \mathrm{~mm}$ probe height (5.4 vs 3.2 secs respectively; $p<0.05$ ) (Fig.6+7). No significant differences were observed between the Pre-Sham-SCS group and the Sham$\mathrm{SCS}$ group ( $\mathrm{F} 1=63.02 ; p=0.481 ; \mathrm{n}=2)$.

\section{Area Between the Curves}

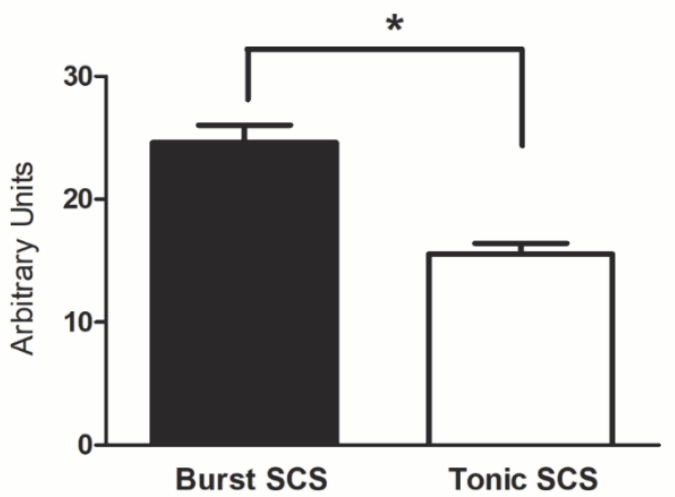

Fig. 8: Area Between the Curves for escape latency for the Pre-Tonic SCS group and Tonic SCS group $(n=5)$ and for the Pre-Burst SCS group and Burst SCS group $(n=5)$. Area Between the Curve $(A B C)$ analysis revealed a significant difference between the $A B C$ for Pre-Burst and Burst and the $A B C$ for Pre-Tonic and Tonic SCS (24.6 vs 15.4 respectively; $p=0.0225$ ). 


\section{Discussion}

Our first objective was to assess the effect of the partial sciatic nerve ligation (PSNL) rat model for chronic neuropathic pain on exit latency in the MCAS. A significant difference at $4 \mathrm{~mm}$ probe height between exit latencies of neuropathic animals and sham-animals, demonstrates that the MCAS-setup is a valid operant testing method for the assessment of affective-motivational aspects of pain in neuropathic PSNL-rats. The second objective was to assess the cognitive-motivational aspects of Tonic- and Burst SCS-induced pain relief, by means of exit latency in the MCAS. This revealed significant differences in cognitivemotivational behavior for Burst-SCS and Tonic-SCS, and these differences could not be detected by reflex-based von Frey testing. Burst-SCS furthermore seems to specifically modulate, much more than Tonic-SCS, cognitive-motivational aspects of pain behavior.

\section{MCAS Exit Latency}

this study, it was demonstrated how latency to exit from the light compartment in the MCAS was significantly increased in chronic neuropathic PSNL-rats, indicating a stimulus-response relationship. At $4 \mathrm{~mm}$ probe height PSNL-animals required significantly more time to exit the light compartment, compared with sham animals. In this respect, use of $4 \mathrm{~mm}$ probe height provides the most optimal window, allowing for discrimination between injured versus sham control animals and thus, for assessment of peripheral neuropathic pain. These findings are in line with those reported by Harte et al. (2016): here a similar significant difference in exit latency at $4 \mathrm{~mm}$ probe height was noted between naïve animals and $\mathrm{CCl}$ animals [13]. At $5 \mathrm{~mm}$ probe height, we observed a sharp drop in exit latency for PSNLanimals. Unfortunately, in the study performed by Harte et al. 2016, results from $5 \mathrm{~mm}$ probeheight were not presented. Therefore, we were unable to compare data at $5 \mathrm{~mm}$ probeheight with previous literature. Since we presented the probeheights in incremental order there is the possibility that the PSNL-animals went through a learning curve. Hence, at the fifth probeheight, the "cost-benefit" analysis including the level of ongoing pain, the height of the probes, and the averseness of the light might have been overruled by a primal instinct to carry through with the oncoming painful stimulus as quickly as possible. Another important difference between our findings and the findings of Harte and colleagues is that the animals in our study exhibit overall lower exit latencies. This could be related to the fact that Harte and colleagues performed their experiments in the inactive (light) phase of the rodent circadian rhythm, as compared to our experiments which were performed during the active (dark) phase [21]. 
In 2008, Vierck and colleagues already addressed the relevance of operant testing, after reviewing several dissociations between reflex-based testing methods and operant testing methods. Interestingly, after closer examination of the aforementioned dissociations they discovered that operant testing more often resulted in a clinically concordant outcome [1, 22]. It is furthermore known that various experimental manipulations do not affect reflex and escape responses in a similar manner, including but not limited to, morphine, naloxone, stress, subcutaneous formalin injection, cutaneous application of mustard oil, and chronic constrictive nerve injury [22-27]. Therefore, although reflex-based testing has contributed in many ways to the preclinical SCS field, it has become apparent that analysis of cognitivemotivational aspects of pain needs to be included. Thus, the MCAS may complement current behavioral testing methods [13]. In the MCAS, for exit latency, the animal is forced to choose between two opposing motivational drives: 1) escape an aversive, yet nonnoxious setting (light compartment) by subjecting itself to noxious stimuli (the nociceptive probes), or 2) avoid the noxious probes but remain in the aversive bright light compartment [13]. This process requires a cost-benefit analysis, which requires input from cortical areas that process objective aspects of pain. In line with this, we made several observations of animals investigating the nociceptive probes with their forelimbs right before the exit process in the MCAS testing system. Thus, the fact that MCAS exit latency testing may recruit ascending pathways creates an opportunity to assess the involvement of supraspinal elements, for instance, as suggested with Tonic-SCS and in particular with Burst-SCS. From a broader perspective, including operant testing methods in preclinical assessment batteries could provide a valid measurement for the cognitive-motivational aspects of pain, which in its turn could prove to be an answer to the lack of translational progress in the pain field [1].

\section{MCAS Exit Latency and Spinal Cord Stimulation}

When Tonic and Burst SCS were administered 30 minutes prior, and during MCAS-testing, a significant overall decrease in exit latency was observed for both Tonic and Burst SCS. Furthermore, at $4 \mathrm{~mm}$ probe height and $5 \mathrm{~mm}$ probe height the Tonic SCS group and the Burst SCS group significantly differed. The latter seems to suggest that Burst SCS has a more profound cognitive-motivational effect when the severity of the nociceptive stimulus is increased. Animals that received sham SCS showed no variations in exit latencies. In the clinical setting, questionnaires regarding quality of life and patients' preference tend to lean towards Burst SCS, as compared to other SCS-waveforms [28-30]. However, these days, there is still no clear consensus in literature, neither preclinical $[4,6,7]$ nor clinical $[2,3,28$ - 
37], regarding the objective analgesic efficacy of Burst SCS (as measured on the VAS-scale), as compared to other SCS-waveforms. A recent clinical study by Kriek and colleagues has shown that the preferred stimulation setting is not solely driven by the amount of pain reduction, but is also influenced by which stimulation setting feels most comfortable and provides the best user-friendliness [35]. Therefore, it is pivotal that we aim to elucidate the (supraspinal) mechanisms responsible for the level of comfort, the sensation, that accompanies SCS-waveforms. As the MCAS can provide critical insight into cognitivemotivational processing of SCS, it could serve as a preclinical tool that allows for the optimization of supraspinal mechanisms of Burst-SCS. It is important to note that the difference between Tonic SCS and Burst SCS, as observed for the exit latencies, was not present in the reflex-based Von Frey analysis. This suggests that the MCAS allows for assessment of behavioral changes in pain that are not detected by reflex-based testing. Interestingly, this is in line with clinical observations that show a similar effect for Burst SCS and Tonic SCS on objective measures such as the VAS-scale (no clinically relevant difference) [30]. Yet, a subset of patients expresses a preference for Burst SCS, over Tonic SCS, due to combination of improved psychological (cognitive-motivational) pain-aspects and the absence of unpleasant perceptions such as paresthesia [3, 28, 30, 31, 33-35, 37]. The supraspinal properties of Burst SCS were first discovered in 2013 when it was reported that Burst SCS could decrease the amount of attention patients paid to pain, in a statistically significant way, while Tonic SCS did not show a similar decrease [3]. This was in 2016 corroborated by a source-localized EEG-study that demonstrated how Burst SCS was able to normalize the 'pain supporting-suppressing balance' by having a greater effect on the dorsal anterior cingulate cortex, as compared to Tonic SCS [2]. From the aforementioned studies it was hypothesized that Burst SCS had a specific supraspinal effect on areas associated with the medial pain system, a system associated with the affective components of the pain experience $[3,38,39]$. Thus, it seems that Burst-SCS not only modulates the unpleasant sensory aspects of pain, but also affects the cognitive-motivational, mostly supraspinal, aspects of pain. Therefore, it is important that we now have a tool, the MCAS, which allows us to adequately assess these supraspinal aspects in a preclinical setting. A better understanding of the supraspinal aspects will allow further analyzing and optimizing of the emotional/motivational properties of the Burst-SCS-protocol. In conclusion, combined use of reflex-based von Frey analysis and the MCAS operant testing method provides us with the opportunity to work towards an optimal balance between sensory aspects and cognitive-motivational aspects of SCS-induced pain relief [40]. 


\section{Limitations}

Only male rats were used in order to prevent a potential bias associated with sex-related differences in neuropathic pain development in rats [41], and to avoid a potential bias related to ovarian sex steroid-induced anti-nociception in female rats [42]. Second, in contrast to Harte et al. 2016, we chose to present the various probe heights in incremental order, as previous studies have shown that initial exposure to extreme stimuli can result in large data variability, which can have a deterrent effect [43].

\section{Conclusion}

We conclude that the MCAS is a valid and reproducible method for the assessment of SCSinduced cognitive-motivational behavioral aspects of pain relief. Use of the MCAS operant testing method revealed significant differences in cognitive-motivational behavior for Burst SCS and Tonic SCS, and this difference could not be detected with reflex-based Von Frey testing. Our behavioral findings strongly suggest that Burst-SCS specifically affects, much more than Tonic-SCS, the processing of cognitive-motivational aspects of pain. 


\section{References}

1. Mogil, J.S., Animal models of pain: progress and challenges. Nat Rev Neurosci, 2009. 10(4): p. 283-94.

2. De Ridder, D. and S. Vanneste, Burst and Tonic Spinal Cord Stimulation: Different and Common Brain Mechanisms. Neuromodulation, 2016. 19(1): p. 47-59.

3. De Ridder, D., et al., Burst spinal cord stimulation for limb and back pain. World Neurosurg, 2013. 80(5): p. 642-649 e1.

4. Gong, W.Y., L.M. Johanek, and K.A. Sluka, A Comparison of the Effects of Burst and Tonic Spinal Cord Stimulation on Hyperalgesia and Physical Activity in an Animal Model of Neuropathic Pain. Anesth Analg, 2016.

5. Sato, K.L., et al., Spinal cord stimulation reduces hypersensitivity through activation of opioid receptors in a frequency-dependent manner. Eur J Pain, 2013. 17(4): p. 551-61.

6. Meuwissen, K.P.V., et al., Burst Spinal Cord Stimulation in Peripherally Injured Chronic Neuropathic Rats: A Delayed Effect. Pain Pract, 2018.

7. Meuwissen, K.P.V., et al., Conventional-SCS vs. Burst-SCS and the Behavioral Effect on Mechanical Hypersensitivity in a Rat Model of Chronic Neuropathic Pain: Effect of Amplitude. Neuromodulation, 2018. 21(1): p. 19-30.

8. van Beek, M., et al., Spinal cord stimulation in experimental chronic painful diabetic polyneuropathy: Delayed effect of High-frequency stimulation. Eur J Pain, 2016.

9. Truin, M., et al., Successful pain relief in non-responders to spinal cord stimulation: The combined use of ketamine and spinal cord stimulation. European Journal of Pain, 2011. 15(10).

10. Truin, M., et al., Increased efficacy of early spinal cord stimulation in an animal model of neuropathic pain. Eur J Pain, 2011. 15(2): p. 111-7.

11. Smits, H., et al., Effect of spinal cord stimulation in an animal model of neuropathic pain relates to degree of tactile "allodynia". Neuroscience, 2006. 143(2): p. 541-6.

12. Smits, H., M. van Kleef, and E.A. Joosten, Spinal cord stimulation of dorsal columns in a rat model of neuropathic pain: evidence for a segmental spinal mechanism of pain relief. Pain, 2012. 153(1): p. 177-83.

13. Harte, S.E., et al., Mechanical Conflict System: A Novel Operant Method for the Assessment of Nociceptive Behavior. PLoS One, 2016. 11(2): p. e0150164.

14. Coy-Laboratory, Coy Mechanical Conflict-Avoidance System for rats (Harte, Morrow method): Reference Manual. 2013.

15. Lau, D., et al., Herpes simplex virus vector-mediated expression of interleukin-10 reduces below-level central neuropathic pain after spinal cord injury. Neurorehabil Neural Repair, 2012. 26(7): p. 889-97.

16. De Ridder, D. and S. Vanneste, Does Tonic Spinal Cord Stimulation Really Influence the Medial Pain System? Neuromodulation, 2016. 19(2): p. 227-8.

17. Seltzer, Z., R. Dubner, and Y. Shir, A novel behavioral model of neuropathic pain disorders produced in rats by partial sciatic nerve injury. Pain, 1990. 43(2): p. 205-18.

18. Chaplan, S.R., et al., Quantitative assessment of tactile allodynia in the rat paw. J Neurosci Methods, 1994. 53(1): p. 55-63.

19. Cui, J.G., et al., Spinal cord stimulation attenuates augmented dorsal horn release of excitatory amino acids in mononeuropathy via a GABAergic mechanism. Pain, 1997. 73(1): p. 87-95.

20. Mills, C., et al., Estimating efficacy and drug ED50's using von Frey thresholds: impact of weber's law and log transformation. J Pain, 2012. 13(6): p. 519-23.

21. Benstaali, C., et al., Circadian rhythms of body temperature and motor activity in rodents their relationships with the light-dark cycle. Life Sci, 2001. 68(24): p. 2645-56.

22. Vierck, C.J., P.T. Hansson, and R.P. Yezierski, Clinical and pre-clinical pain assessment: are we measuring the same thing? Pain, 2008. 135(1-2): p. 7-10. 
23. King, C.D., et al., Opioid modulation of reflex versus operant responses following stress in the rat. Neuroscience, 2007. 147(1): p. 174-82.

24. Vierck, C.J., A.J. Acosta-Rua, and R.D. Johnson, Bilateral chronic constriction of the sciatic nerve: a model of long-term cold hyperalgesia. J Pain, 2005. 6(8): p. 507-17.

25. Vierck, C.J., et al., Low dose systemic morphine attenuates operant escape but facilitates innate reflex responses to thermal stimulation. J Pain, 2002. 3(4): p. 309-19.

26. Vierck, C.J., Jr., J.D. Greenspan, and L.A. Ritz, Long-term changes in purposive and reflexive responses to nociceptive stimulation following anterolateral chordotomy. J Neurosci, 1990. 10(7): p. 2077-95.

27. Vierck, C.J., Jr., R.H. Kline, and R.G. Wiley, Intrathecal substance $p$-saporin attenuates operant escape from nociceptive thermal stimuli. Neuroscience, 2003. 119(1): p. 223-32.

28. De Ridder, D., et al., A 2-center comparative study on tonic versus burst spinal cord stimulation: amount of responders and amount of pain suppression. Clin J Pain, 2015. 31(5): p. 433-7.

29. Vesper, J., et al., Burst SCS Microdosing Is as Efficacious as Standard Burst SCS in Treating Chronic Back and Leg Pain: Results From a Randomized Controlled Trial. Neuromodulation, 2018.

30. Deer, T., et al., Success Using Neuromodulation With BURST (SUNBURST) Study: Results From a Prospective, Randomized Controlled Trial Using a Novel Burst Waveform. Neuromodulation, 2018. 21(1): p. 56-66.

31. de Vos, C.C., et al., Burst spinal cord stimulation evaluated in patients with failed back surgery syndrome and painful diabetic neuropathy. Neuromodulation, 2014. 17(2): p. 152-9.

32. De Ridder, D., et al., Burst spinal cord stimulation: toward paresthesia-free pain suppression. Neurosurgery, 2010. 66(5): p. 986-90.

33. Demartini, L., et al., Comparison of Tonic vs. Burst Spinal Cord Stimulation During Trial Period. Neuromodulation, 2018.

34. Tjepkema-Cloostermans, M.C., et al., Effect of Burst Stimulation Evaluated in Patients Familiar With Spinal Cord Stimulation. Neuromodulation, 2016. 19(5): p. 492-7.

35. Kriek, N., et al., Preferred frequencies and waveforms for spinal cord stimulation in patients with complex regional pain syndrome: A multicentre, double-blind, randomized and placebocontrolled crossover trial. Eur J Pain, 2016.

36. Verrills, P., C. Sinclair, and A. Barnard, A review of spinal cord stimulation systems for chronic pain. J Pain Res, 2016. 9: p. 481-92.

37. Hou, S., K. Kemp, and M. Grabois, A Systematic Evaluation of Burst Spinal Cord Stimulation for Chronic Back and Limb Pain. Neuromodulation, 2016. 19(4): p. 398-405.

38. Kulkarni, B., et al., Attention to pain localization and unpleasantness discriminates the functions of the medial and lateral pain systems. Eur J Neurosci, 2005. 21(11): p. 3133-42.

39. Basbaum, A.I., et al., Cellular and molecular mechanisms of pain. Cell, 2009. 139(2): p. 267-84.

40. (IASP), I.a.f.t.s.o.p., Part III: Pain Terms, A Current List with Definitions and Notes on Usage. IASP Task Force on Taxonomy, 1994.

41. Devall, A.J., Z.W. Liu, and T.A. Lovick, Hyperalgesia in the setting of anxiety: sex differences and effects of the oestrous cycle in Wistar rats. Psychoneuroendocrinology, 2009. 34(4): p. 587-96.

42. Liu, N.J. and A.R. Gintzler, Prolonged ovarian sex steroid treatment of male rats produces antinociception: identification of sex-based divergent analgesic mechanisms. Pain, 2000. 85(12): p. 273-81.

43. Sotocinal, S.G., et al., The Rat Grimace Scale: a partially automated method for quantifying pain in the laboratory rat via facial expressions. Mol Pain, 2011. 7: p. 55. 


\title{
CHAPTER 6
}

\section{The Supraspinal Mechanisms of Burst Spinal Cord Stimulation: a Functional Magnetic Resonance Imaging Study with Peripherally Injured Chronic Neuropathic Rats.}

\author{
Koen P.V. Meuwissen*1,2 ${ }^{2}$ Annette van der Toorn ${ }^{3}$, Rick M. Dijkhuizen ${ }^{3}$,
} Elbert A. J. Joosten ${ }^{1,2}$.

Submitted to the journal of anesthesiology

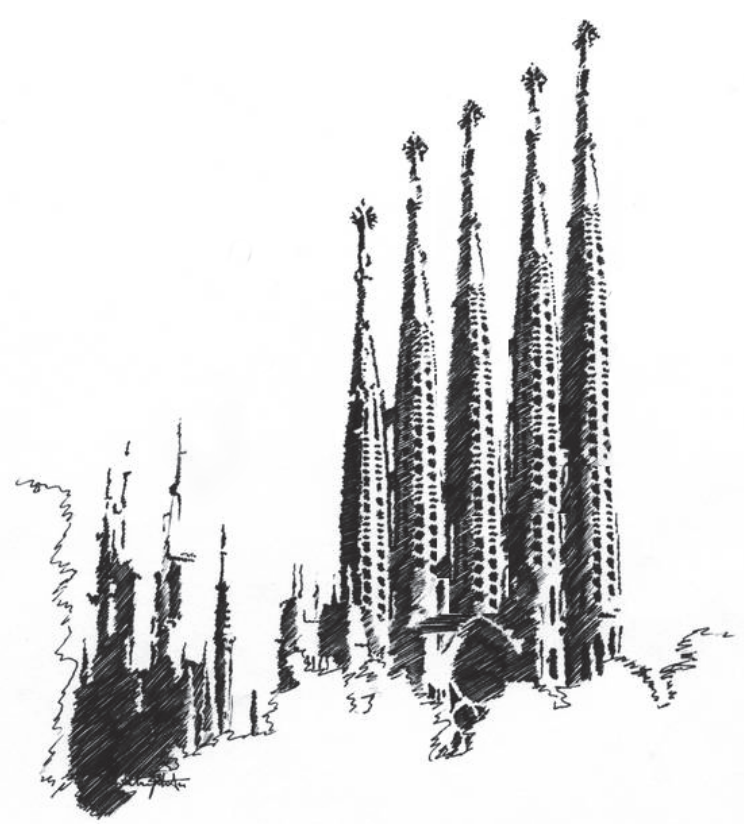




\section{Abstract}

Introduction: Spinal Cord Stimulation (SCS) is a last-resort treatment option for a variety of intractable neuropathic pain disorders. Pain relief induced by Tonic SCS acts via local spinal activation and is known to include supraspinal activation of sensorimotor brain areas. It is suggested that a more recently introduced mode of SCS, Burst SCS, modulates cognitivemotivational aspects of pain. In order to understand supraspinal mechanisms and the different aspects of pain relief induced with Tonic or Burst-SCS we used functional magnetic resonance imaging ( $\mathrm{fMRI}$ ) in chronic neuropathic rats. Based on literature, we hypothesized that both Tonic SCS and Burst SCS would result in increased BOLD-signal in brain regions associated with location and intensity of pain, and cognitive-emotional aspects of pain. Furthermore, we also hypothesized that Burst SCS would induce a more profound BOLDincrease in brain areas associated with cognitive-emotional aspects of pain, as compared to Tonic SCS.

Methods: Sprague Dawley Rats $(n=17)$ received a unilateral partial sciatic nerve ligation which resulted in chronic neuropathic pain. Two weeks after the ligation quadripolar SCSelectrodes were epidurally positioned on top of the dorsal columns at T13. Isofluraneanesthetized (1.5\%) rats received either Tonic-SCS $(n=8 ; 200 \mu$ s pulse width, $50 \mathrm{~Hz})$ or BurstSCS ( $n=9 ; 5$ pulses, 1 ms pulse width, $449 \mathrm{~Hz}$ intra-burst, $40 \mathrm{~Hz}$ inter-burst) at $66 \%$ of motorthreshold (MT). BOLD FMRI was conducted pre-, during and post-SCS using a 9.4T horizontal bore scanner (Varian, Palo Alto, CA, USA). SCS was applied in a block-design, which consisted of three times 5 minutes of SCS, alternated with three times 5 minutes resting period.

Results: Overall, Tonic SCS resulted in a moderate increase of BOLD signal levels in areas associated with the location and intensity of pain such as the primary somatosensory cortex $(z=2.7)$, and the premotor cortex $(z=2.8)$ and areas associated with cognitive-emotional aspects of pain such as the anterior cingulate cortex ( $z=3.2)$, amygdala ( $z=3.2)$ and insular cortex ( $z=4.4)$. Burst SCS resulted in a larger and more robust increase of BOLD signal levels in areas associated with the location and intensity of pain such as the primary somatosensory cortex $(z=12.8)$, and the premotor cortex $(z=14.2)$ and areas associated with cognitive-emotional aspects of pain such as the anterior cingulate cortex $(z=15.1)$, amygdala $(z=11.9)$ and insular cortex $(z=18.4)$. Additionally, Burst SCS increased BOLD-signal in the raphe nuclei $(z=8.1)$, and areas associated with the reward system such as the nucleus accumbens $(z=13.4)$, and the caudate putamen $(z=17.1)$. Tonic SCS did not induce a significant increase in BOLD-amplitude in these areas. 
Conclusions: Burst and Tonic SCS have different effects on the intensity and localization of SCS-induced changes in BOLD-levels in the brain. Overall, both Tonic SCS- and Burst SCS increased BOLD-signal in brain regions associated with location and intensity of pain, and cognitive-emotional aspects of pain. However, Burst SCS induced a more profound BOLDincrease in brain areas associated with cognitive-emotional aspects of pain, as compared to Tonic SCS. 


\section{Introduction}

Damage or disease affecting the somatosensory nervous system may result in chronic intractable neuropathic pain: a debilitating physical status that greatly influences the quality of life. Over the last two decades, Tonic spinal cord stimulation (SCS) with a frequency within the range of $40-80 \mathrm{~Hz}$ and a pulse width in between 200-500 $\mu \mathrm{Sec}$ [1-3], has been successfully applied as a last-line treatment option for patients with refractory chronic pain syndromes such as failed back surgery syndrome [4-6], complex regional pain syndrome [716] and painful diabetic polyneuropathy [17-19]. Electrical stimulation of the dorsal column with Tonic SCS results in action potentials propagating, antidromically, via A $\beta$-fibers into the spinal cord nociceptive network [20-22]. The antidromic stimulation of $A \beta$-fibers results in changed (decreased) release of glutamate, and at the same time, an increased release of GABA [23-25]. At the same time, action potentials travel orthodromically via the A $\beta$-fibers in rostral direction to supraspinal centers [26]. Early clinical fMRI-work on the supraspinal effects of Tonic SCS has shown modulation of brain regions associated with the lateral spinothalamic tract (STT) [27-29]. The lateral STT is responsible for the transmission of objective pain-aspects such as the intensity and location of the painful stimulus. This pathway projects via the thalamus to cortical areas such as the somatosensory cortex [30, 31]. An fMRI study performed in eight patients receiving Tonic SCS demonstrated that Tonic stimulation increased BOLD-levels in somatosensory cortices, sensorimotor cortex and the insula. Furthermore, it was reported that Tonic SCS induced decreased BOLD-signal in the primary motor cortices and the primary somatosensory cortex [28]. A more recent fMRIstudy, with twenty patients who received Tonic-SCS treatment for FBSS, reported deactivation of the bilateral medial thalamus and its connections to the rostral and caudal cingulate cortex and the insula [27]. Interestingly, Deogaonkar and colleagues recently reported a decreased connection strength between somatosensory and limbic areas with Tonic SCS. This led them to conclude that pain relief from Tonic SCS may also reduce negative emotional processing associated with pain [32]. Recently, a novel SCS-mode has been introduced able to specifically modulate cognitive-emotional aspects of pain with periodic bursts of electric pulses [33, 34]. Electroencephalography-analysis of 5 patients who received the SCS-mode, known as Burst SCS, showed a greater normalization of the pain supporting/ suppressing balance, and with that better modulation of cognitiveemotional aspects of pain, as compared to Tonic stimulation mode [35]. In 2013, a small clinical study demonstrated that patients who received Burst SCS significantly improved on cognitive-emotional pain-aspects like 'the amount of attention the patients paid to pain' 
and 'changes in pain' (measured by the Pain Vigilance and Awareness Questionnaire (PVAQ)) [33]. Meanwhile, patients who received Tonic or placebo stimulation did not show significant differences on the cognitive-emotional pain-aspects. From this, it was suggested that Burst SCS might activate both the lateral and the medial STT; as the latter is known to contribute specifically to the emotional affective aspects of pain via the cingulate and insular cortices and its connections in the brainstem (parabrachial nucleus) and amygdala $[31,35]$. Nevertheless, the mechanisms underlying the pain relieving effect of Burst SCS remain largely unknown, especially on a supraspinal level, and elucidating the cognitive/emotion-modulating effects of Burst SCS could aid its optimization process. Therefore, we designed an experimental study on SCS-induced pain relief in chronic neuropathic animals in which we assessed the Blood-Oxygenation-Level Dependent (BOLD) activation patterns in supraspinal brain regions both for both Burst-SCS and for Tonic-SCS. Based on literature, we hypothesized that both Tonic SCS- and Burst SCS-induced pain relief in chronic neuropathic animals would result in increased BOLD-signal in brain regions associated with location and intensity of pain, and cognitive-emotional aspects of pain. Furthermore, we also hypothesized that Burst SCS would induce a more profound BOLDincrease in brain areas associated with cognitive-emotional aspects of pain, as compared to Tonic SCS. 


\section{Methods and Materials}

\section{Ethics Statement}

All experiments were performed in accordance with the European Directive for the Protection of Vertebrate Animals Used for Experimental and Other Scientific Purposes (86/609/EU). The protocol was approved by the Animal Research Committee of the Maastricht University Medical Centre (DEC-protocol 2014-086).

\section{Animals}

All experiments were performed using male Sprague Dawley rats $(n=19)$, which were young-adult ( 5 weeks of age) at the start of the experiment (150-200 g). Animals were housed as groups of 2 , in polycarbonate cages in a climate-controlled vivarium maintained under controlled temperature $\left(21 \pm 1^{\circ} \mathrm{C}\right)$, relative humidity $(55 \pm 15 \%)$ and artificial lighting (12:12 light/dark cycle) with distilled water and rodent chow available ad libitum. All procedures were conducted between 08:00 and 18:00 hours during the light phase of the animal facility.

\section{Partial Sciatic Nerve Ligation (PSNL)}

A unilateral ligation of the left sciatic nerve was performed as described by Seltzer et al. 1990 [36], as previously applied in our laboratory [37-40]. In short, animals were anesthetized with 3-5\% isoflurane (Abbott Laboratories Ltd., Kent, U.K.). The left sciatic nerve was exposed by blunt dissection and carefully freed from surrounding connective tissue. For sham-PSNL animals, the sciatic nerve was left unaffected and the wound was closed with a $4 / 0$ silk suture. For PSNL animals, distal to the posterior biceps semitendinosus, but proximal to the little fat pad that lies a few millimeters distal to this site, the sciatic nerve was partially ligated. An 8/0 non-absorbable silk suture was used to ligate approximately $1 / 3$ of the diameter of the left sciatic nerve. After ligation, the wound was closed with a 4/0 silk suture. The presence of mechanical hypersensitivity was considered successful if at 13 days post-surgery, paw withdrawal thresholds to Von Frey stimuli $\left({ }^{10} \log (50 \%)\right)$ were decreased by 0.2 units compared with baseline (day 0) [37-40].

\section{Assessment of mechanical hypersensitivity (von Frey assay)}

Assessment of paw withdrawal threshold (PWT) was performed according to the protocol described in Meuwissen et al.[39, 40]. PWT was assessed according to the up-down method' [41]. The 50\% PWT was calculated after completion of a sequence of six 
consecutive responses. A cut-off value of $28.84 \mathrm{~g}$ was defined. For statistical analysis, $50 \%$ WTs were logarithmically transformed to obtain a linear scale.

\section{Implantation of spinal cord stimulation device}

The implantation of the SCS device was performed according to the protocol which is applied as standard implantation procedure in our institution [38-40, 42, 43], based on, and identical to the method originally developed at the Karolinska Institute [44]. In short, a small laminectomy was made at the thoracic level 13 (Th13). The laminectomy was followed by careful exposure the spinal cord by use of a surgical rotary tool. Subsequently, a custommade cylindrical 4-contact lead $(0.72 \mathrm{~mm}$ diameter; Boston Scientific Neuromodulation, Valencia, CA, USA) was inserted into the epidural space on top of the spinal cord and then carefully positioned in the caudal direction. Electrode configuration was set at alternating anode and cathode settings (rostral to caudal: + - +-). Lastly, the electrode was secured to a spinous process with tissue adhesive (Histoacryl ${ }^{\circledR}$, B Braun Medical BV, Oss, the Netherlands) to prevent electrode migration. Before the wound was closed, electrode wires were tunneled subcutaneously to the animal's hips and the stimulator-connectors were attached to the skin. After implantation of the electrodes, the rats were given 2 days for recovery prior to the initiation of SCS.

\section{Spinal cord stimulation}

Spinal cord stimulation (SCS) was performed according to the protocol described in Meuwissen et al. [40]. The Arduino Uno stimulator was set to deliver constant current biphasic stimulation for both the Tonic and Burst SCS modes. Tonic SCS Motor Threshold (MT) was determined at the following settings: pulse width of $200 \mu \mathrm{S}$ administered at a frequency of $2 \mathrm{~Hz}$. Burst SCS MT was determined at the following settings: pulse width of $1000 \mu \mathrm{S}$, five pulses ( $449 \mathrm{~Hz}$ intra-burst frequency) administered at an inter-burst frequency of $2 \mathrm{~Hz}$. The amplitude was gradually increased until symmetrical contractions of the hind limbs were perceived by hand and/or visually observed. Then, either a Tonic SCS paradigm at $66 \%$ of MT (frequency $50 \mathrm{~Hz}$, pulse width $200 \mu \mathrm{S}$ ) [23], or a biphasic Burst-SCS paradigm at $66 \% \mathrm{MT}$ (inter-burst-frequency $40 \mathrm{~Hz}$, pulse width $1000 \mu \mathrm{S}, 5$ spikes at $449 \mathrm{~Hz}$ intra-burst frequency) was applied for 60 minutes [39, 40]. 


\section{MRI acquisition}

All MR experiments were performed in a 9.4 T horizontal bore scanner (Varian, Palo Alto, CA, USA) equipped with a $20.5 \mathrm{~cm}$ gradient with gradient strengths up to $400 \mathrm{mT} / \mathrm{m}$. An actively decoupled and inductively coupled custom-made surface coil (diameter $25 \mathrm{~mm}$ ) was used for reception of the MR signal and for excitation an Helmholtz design actively decoupled excitation coil (diameter $80 \mathrm{~mm}$ ) was used. Rats were intubated and actively ventilated while restrained in the scanner by a stereotactic holder with ear plugs and a tooth holder. Body temperature was maintained at $37^{\circ} \mathrm{C}$ with a warm water mat. Oxygen saturation and heart rate and end-tidal $\mathrm{CO}_{2}$ were continuously monitored with a pulse oximeter and a capnography respectively. After correct positioning of the rat in the scanner and shimming the isoflurane level was decreased to $1.5 \%$ and anatomical images were obtained using a balanced gradient echo sequence (TR/TE $5 / 2.5 \mathrm{~ms}$, flip angle $20^{\circ}$, FOV $40 * 32 * 24 \mathrm{~mm}, 160 * 128 * 96$ points, slab $14.4 \mathrm{~mm}, \mathrm{nt}=3$, repeated 4 times with pulse angle shifts of $0^{\circ}, 90^{\circ}, 180^{\circ}$ and $270^{\circ}$ ). This acquisition took about 12 minutes and 18 seconds in which the new isoflurane level could be established. The anatomical scan was followed by a resting state $\mathrm{fMRI}$ acquisition of 800 images using a gradient-echo EPI sequence in which the phase-encode direction was encoded within the EPI-train and the slice direction was phase-encoded, obtaining a 3D image every $862.4 \mathrm{~ms}$ (TR/TE 30.8/16 ms, FOV 36*36*16.8 $\mathrm{mm}^{3}, 60^{*} 60^{*} 28$ points, flip angle $\left.13^{\circ}\right)$. After the resting state $\mathrm{fMRI}$ a series of 660 images was acquired using the same EPI acquisition, where the first 180 images were obtained in rest condition, followed by 300 images with spinal cord stimulation and ending with 180 images without stimulation. This sequence was repeated three times with intervals of about 1 to 2 minutes and followed by a final resting state fMRI acquisition of 800 images.

\section{Experimental design}

The animals received Von Frey testing prior to the partial sciatic nerve ligation (PSNL) as described in section 2.3. After the subsequent 14 day observation period, animals received Von Frey measurements in order to assess the development of mechanical hypersensitivity. All animals that received a unilateral PSNL developed mechanical hypersensitivity measured by von Frey filament assays at day 14 post ligation $(n=19)$. These 19 animals received implantation of an SCS-electrode, as described in section 2.5. Animals for which the SCSelectrode was successfully implanted $(n=19)$ were given two days to recover from the surgery before the Motor-Threshold (MT) was assessed. Prior to MT-assessment, animals were connected to the stimulator as if to receive stimulation. Animals which did not show a robust MT $(n=2)$, hence, did not show clear symmetrical contractions of the hind limbs, 
were excluded from the study and did not receive SCS. The remaining animals ( $n=17)$, were randomly divided over the Tonic-SCS $(n=8)$ or Burst-SCS group $(n=9)$. Animals were placed in the scanner, and received SCS in the scanner as described in section 2.7. Anesthesia was induced with 3-5\% isoflurane in $\mathrm{O}_{2}$ /air (1:4) and animals were endotracheally intubated for mechanical ventilation. Anesthesia during scanning was kept at a maintenance-dose of $1.5 \%$ isoflurane in $\mathrm{O}_{2}$ /air (1:4). Animals were placed in the scanner and attached to an Arduino Uno stimulator by means of a Mu-metal shielded cable set-up connected to the external electrode contacts on the hips. Stimulator software was linked and synchronized with the MRI-acquisition software for adequate timing of stimulation-onset and offset. Animals received SCS in the scanner in a block-design that consisted of three times 5 minutes of SCS, alternated with three times 5 minutes resting period.

\section{Image processing}

FMRIB's Software Library (FSL) v5.0 was used for all MRI analyses, unless mentioned otherwise. After Fourier transforming of the stimulation EPI-data the first 5 images were removed to reduce initial saturation effects. Motion correction was applied using MCFLIRT [45]. A median image was obtained from 155 motion corrected images before start of the stimulation and this image is corrected for image intensity non-uniformity (caused by the surface coil) using the MINC tool N3 [46].This was followed by brain extraction using the BET tool implemented in FSL [47]. Additional erosion and expansion steps were added to improve the brain mask. The resulting median image and its extracted brain mask were used for all later registration steps, including registration of the anatomical image to the fMRI image. The motion-corrected fMRI data were low-pass filtered in the Fourier-domain using a cut-off frequency of $0.1 \mathrm{~Hz}$ using home-made software, after which the first and last 15 images were removed. To create a template for all block-design fMRI data of the experiments, the median image of the first stimulation $\mathrm{fMRI}$ data was registered to a reference fMRI image using the linear registration tool FLIRT $[45,48]$, followed by the nonlinear registration Tool FNIRT $[49,50]$. The second and third stimulation were first linearly registered to the first stimulation data set after which the warp-file of the first stimulation series was used for registration to the reference. A two-level generalized linear model (GLM) analysis was performed. For this, an on-period of 300 images (after 155 images off) convolved with a hemodynamic response function was used as a regressor. This resulted in three activation maps (z-maps) per animal. A lower threshold of 5 was used as cut-off value for the activation maps. To allow analysis of time series data from specific regions of interest (ROIs) a template image for morphometry analysis based on the Paxinos and Watson atlas 
[51] was used $[52,53]$. Non-linear registration (FNIRT) of the fMRI images to the rat anatomical image followed by non-linear registration of the anatomical image to the template image was performed to allow the projection of the ROls defined in the Paxinos and Watson atlas on the fMRI data. The mean signal of the ROI over time was obtained for each rat and each stimulation (activation traces). It was normalized for display by dividing the signal with the mean of the first 150 images. Activation traces were also used as input for a two-level GLM analysis and the resulting z-values (three per animal) were analyzed.

\section{Data Analysis}

The WTs to von Frey filaments are presented as mean \pm standard error of the mean (SEM). For statistical analysis Von Frey data were logarithmically transformed to obtain a linear scale and to account for Weber's Law [54]. For the analysis of differences in the withdrawal thresholds between groups, ipsilateral and contralateral withdrawal thresholds were compared using paired-sampled T-tests. All statistical analyses were performed with $\alpha=$ 0.05 using IBM SPSS statistics 23. Analysis of BOLD-Amplitude was conducted on normalized data by means of a Two-Way ANOVA with Bonferroni Post-hoc analysis. Data for each ROI was averaged for all animals of each stimulation-protocol, and subsequently compared for the Tonic-SCS group and Burst-SCS group. Two-Way ANOVA with Bonferroni Post-hoc analysis was performed for BOLD-Amplitude pre-SCS and during SCS for the Tonic-SCS group and Burst-SCS group. Furthermore, Two-Way ANOVA with Bonferroni Post-hoc analysis was performed for BOLD-Amplitude of the Tonic-SCS group and Burst-SCS group during SCS. 


\section{Results}

\section{Development of mechanical hypersensitivity in chronic neuropathic rats}

Pre-nerve injury, PWTs of the ipsilateral hind paws were comparable with the PWTs of the contralateral hind paws (ipsilateral $15.3 \pm 1.9 \mathrm{~g}$ vs. contralateral $15.0 \pm 1.4 \mathrm{~g}$ ) in all animals (Fig.1A). All animals that received a unilateral PSNL developed mechanical hypersensitivity to stimulation by von Frey filaments (ipsilateral average PWTs: $15.6 \pm 1.7 \mathrm{~g}$ (pre-lesion) vs. $1.6 \pm 0.6 \mathrm{~g}$ (post-lesion); $p=.0045$ ) (Fig $1 \mathrm{~A})$.

\section{Effect of SCS: von Frey analysis}

In animals of the SCS-group ( $n=17$ ) paw withdrawal thresholds (PWTs) to von Frey filaments was assessed before the start of SCS treatment (Tonic or Burst SCS), at 30',60' min. after stimulation was turned on. No significant differences were observed in ipsilateral paw PWTs at baseline, pre-SCS, between groups ( $p=0.61$; Tonic-SCS $1.6 \pm 0.3 \mathrm{~g}[\mathrm{n}=8]$ vs. Burst-SCS 1.7 $\pm 0.6 \mathrm{~g}[\mathrm{n}=9])$. After 30 mins of stimulation, PWTs of both the Burst-SCS group ( $p=0.031 ; 1.7$ $\pm 0.6 \mathrm{~g}$ vs. $12.5 \pm 2.8 \mathrm{~g}$ ) and Tonic-SCS group ( $p=0.038 ; 1.6 \pm 0.3 \mathrm{~g}$ vs. $10.7 \pm 2.9 \mathrm{~g})$ significantly differed from baseline PWTs (Fig.1B). After 60 mins of stimulation, PWTs of both the Burst$\operatorname{SCS}$ group $(p=0.034 ; 1.7 \pm 0.6 \mathrm{~g}$ vs.11.8 $\pm 2.1 \mathrm{~g}$ ) and Tonic-SCS group $(p=0.035 ; 1.6 \pm 0.3 \mathrm{~g}$ vs.11.1 $\pm 2.5 \mathrm{~g}$ ) significantly differed from baseline PWTs (Fig.1B). PWTs of the Burst-SCS group and Tonic SCS group did not significantly differ at 30 mins of stimulation ( $p=0.44$; Tonic-SCS $10.7 \pm 2.9 \mathrm{~g}$ [n=5] vs. Burst-SCS $12.5 \pm 2.8 \mathrm{~g}[\mathrm{n}=5])$ and 60 mins of stimulation $(p=$ 0.67 ; Tonic-SCS $11.1 \pm 2.5 \mathrm{~g}$ vs. Burst-SCS $11.8 \pm 2.1 \mathrm{~g}$ ) (Fig.1B). 
A

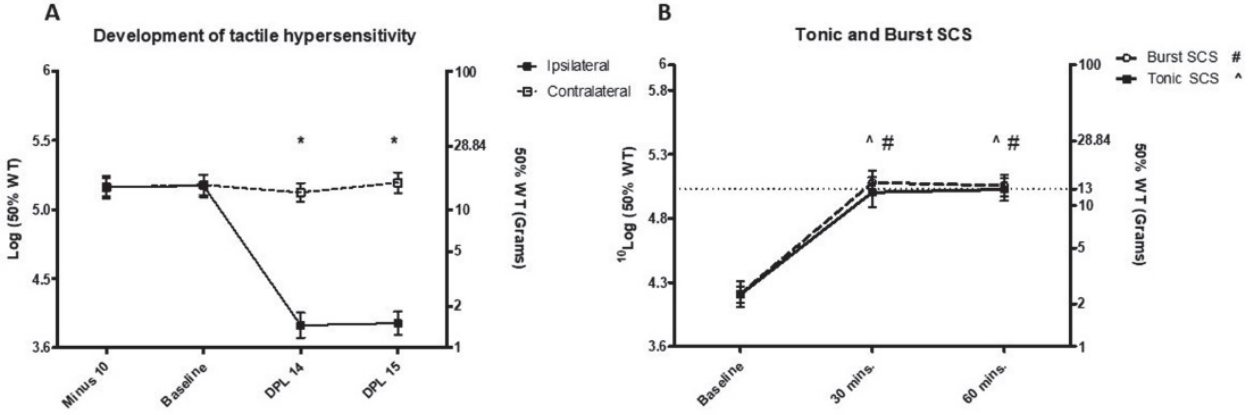

Fig. 1A: Development of mechanical hypersensitivity, based on paw withdrawal threshold s (PWT) and sensitivity to von Frey filaments, after unilateral sciatic nerve ligation in adult rat $(n=17) .{ }^{*} p<0.05$ for ipsilateral versus contralateral. B: The effect of Tonic SCS $(n=8)$ and Burst SCS $(n=9)$ on the paw withdrawal thresholds (PWT) based on sensitivity to von Frey filaments. PWT's were assessed at 30 and 60 mins. of stimulation. The dotted line represents the average WT baseline prior to sciatic nerve ligation. ( $\wedge p<0.05$ for Tonic SCS time points compared to pre-SCS baseline PWTs, \# $p<0.05$ for Burst SCS compared to pre-SCS baseline PWTs).

\section{Spinal Cord Stimulation in the MRI-scanner}

\section{Neural responses to Burst SCS and Tonic SCS}

The group average brain activation patterns in response to Tonic SCS and Burst SCS are shown in Figure 2. Z-scores per stimulation protocol for regions of interest are listed in Table 1. Tonic SCS induced significant brain activation ( $z$-value $>1.96, p<0.05$ ) in regions of interest (ROIs) associated with location and intensity of pain such as the primary somatosensory cortex (hind limb region) $(z=2.7)$, and the premotor cortex $(z=2.8)$ (Figure 2 ; Table 1). Tonic SCS also induced significant brain activation in areas associated with cognitive-emotional aspects of pain such as the anterior cingulate cortex $(z=3.2)$, amygdala $(z=3.2)$ and insular cortex $(z=4.4)$. Burst SCS resulted in a larger and more robust increase of BOLD signal levels in all ROIs, as compared to Tonic SCS. Burst SCS increased BOLD-levels in areas associated with the location and intensity of pain such as the primary somatosensory cortex (hind limb region) $(z=12.8)$, and the premotor cortex $(z=14.2)$ and areas associated with cognitive-emotional aspects of pain such as the anterior cingulate cortex $(z=15.1)$, amygdala $(z=11.9)$ and insular cortex $(z=18.4)$ (Figure 2; Table 1). Additionally, Burst SCS increased BOLD-signal in the dorsal raphe nucleus $(z=8.1)$, and areas associated with the reward system such as the nucleus accumbens $(z=13.4)$, and the caudate putamen $(z=17.1)$. 


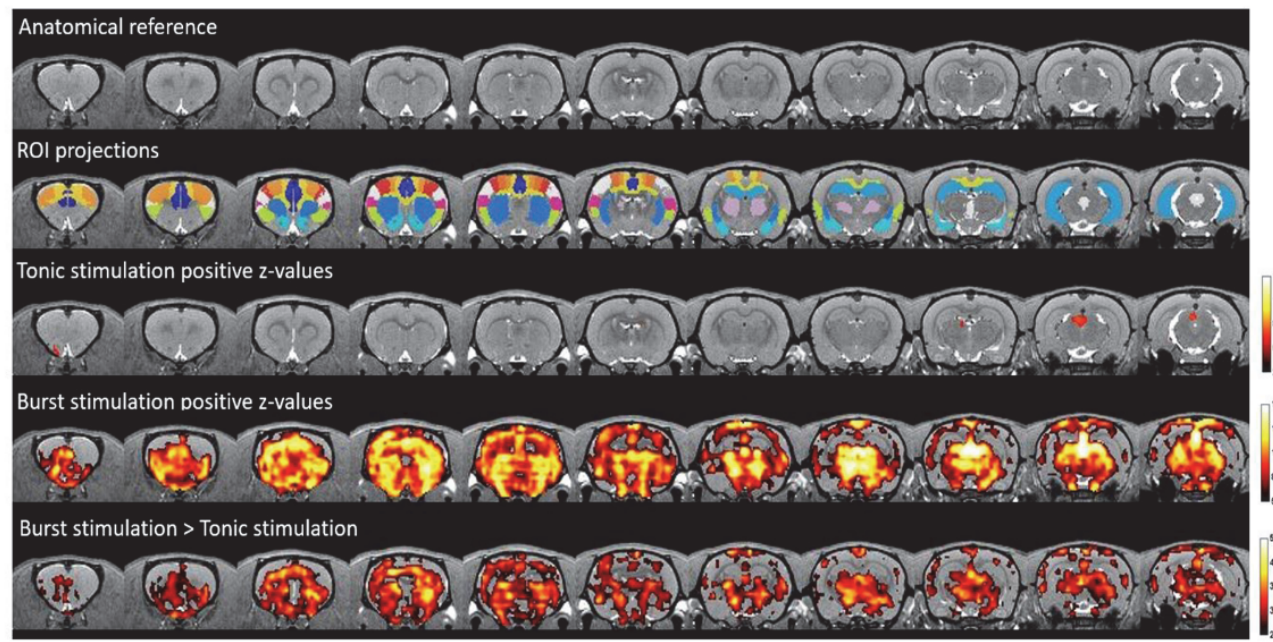

Fig.2: Mean z-values and ROls projected onto reference image for Tonic SCS (third row), Burst SCS (fourth row), and for areas that showed larger BOLD signal changes for Burst SCS as compared to Tonic SCS (fifth row).

\begin{tabular}{|c|c|c|}
\hline Regions of Interest & Tonic SCS Z-scores & Burst SCS Z-scores \\
\hline Anterior Cingulate Cortex & 3.2 & 15.1 \\
\hline Caudate Putamen & 5.4 & 17.1 \\
\hline Hippocampus & 4.2 & 11.7 \\
\hline Nucleus Accumbens & 3.7 & 13.4 \\
\hline Amygdala & 3.2 & 11.9 \\
\hline Raphe Nuclei & 2.2 & 8.1 \\
\hline Insular Cortex & 4.4 & 18.4 \\
\hline Primary Motor Cortex & 3.7 & 15.1 \\
\hline Premotor Motor Cortex & 2.8 & 14.2 \\
\hline Thalamus & 4.8 & 17.4 \\
\hline Periaqueductal Gray & 5.3 & 18.0 \\
\hline S1 Cortex (Hindlimb) & 2.7 & 12.8 \\
\hline S1 Cortex (Forelimb) & 4.2 & 17.4 \\
\hline
\end{tabular}

Table 1: Z-scores for Tonic and Burst stimulation per region of interest. 


\section{BOLD Amplitude Response Curves}

Figure 3 and 4 display the normalized BOLD signal time-course in ROls in brain-regions associated with the processing of location and intensity of pain (Fig.3) and cognitiveemotional aspects of pain (Fig.4). For both the brain-regions associated with the processing of location as well as brain regions associated with the cognitive-emotional aspects of pain Burst SCS induced a more profound increase in BOLD amplitude. Figure 5 displays the normalized BOLD signal time-course in ROIs in brain-regions associated with the processing of reward (caudate putamen and nucleus accumbens) and the raphe nuclei. A strong increase in BOLD amplitude was observed in these regions with Burst SCS, however, this was not observed for Tonic SCS (Fig.5+6, Table 2).
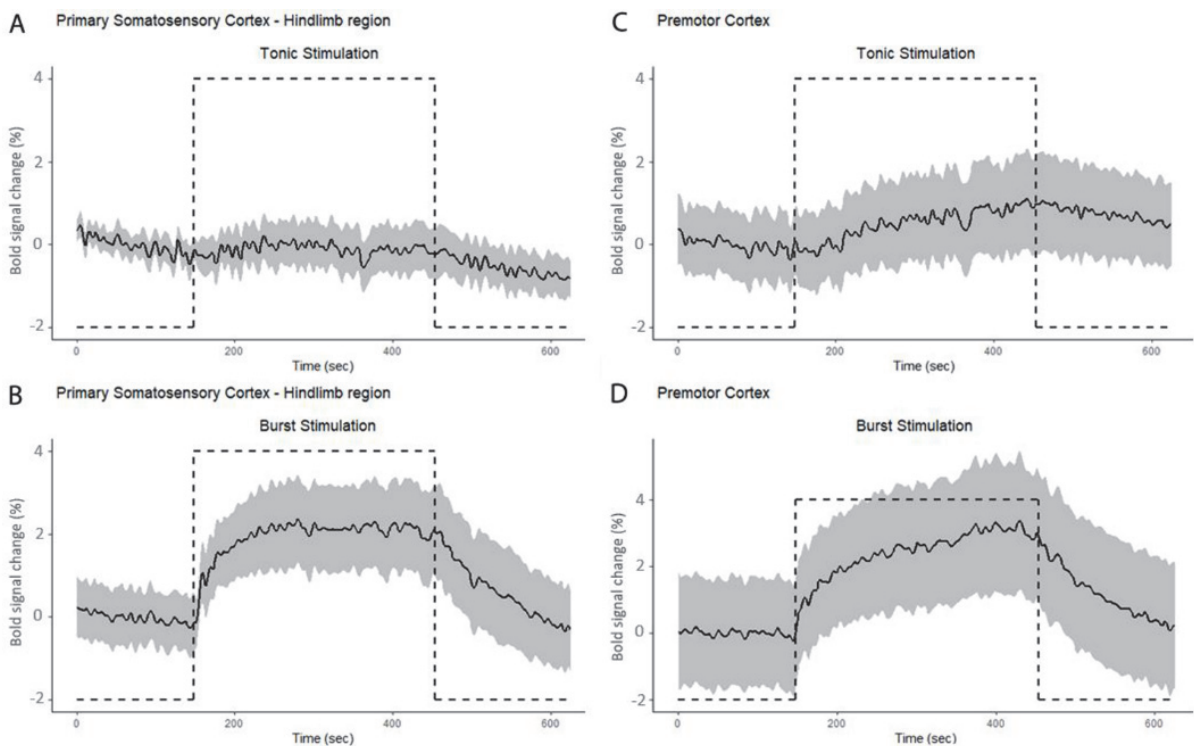

Fig.3: BOLD signal (\%). Average time-courses (mean \pm SEM) from representative brain regions associated with the processing of location and intensity of pain for Tonic SCS $(A+C)$ and Burst SCS $(B+D)$. Figure demonstrates a change of the BOLD signal response during SCS. The stimulus "on" period is shown by the dotted line. 

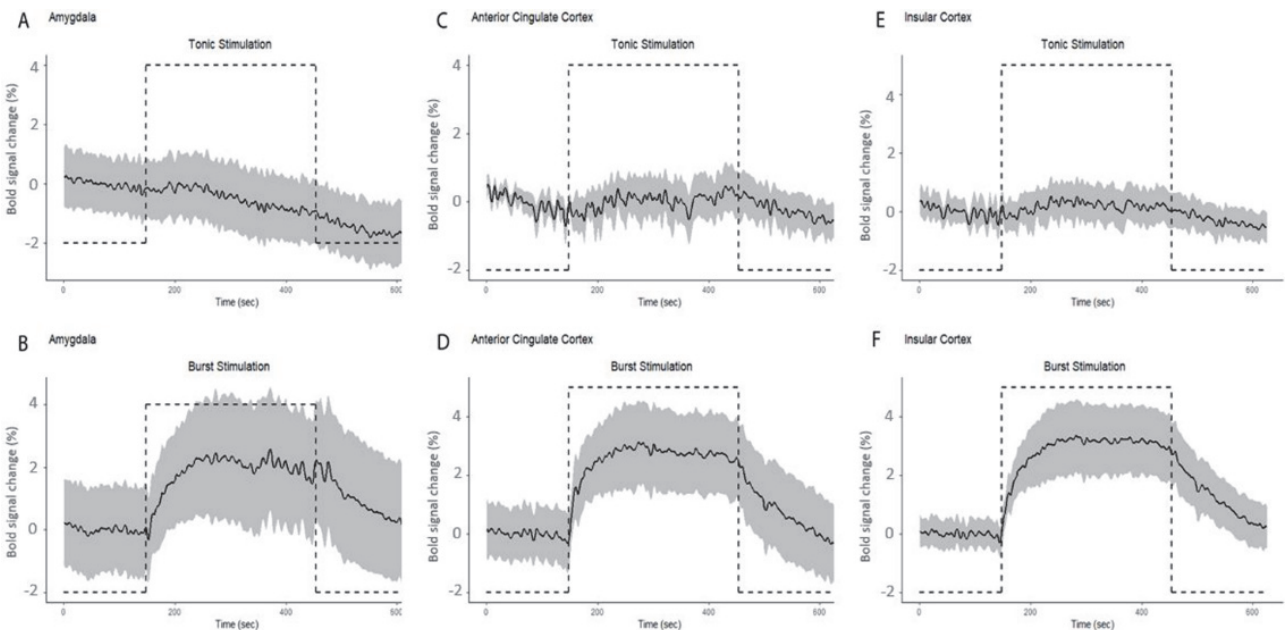

Fig.4: BOLD signal (\%). Average time-courses (mean \pm SEM) from representative brain regions brain regions associated with the processing of cognitive-emotional aspects of pain for Tonic SCS $(A+C+E)$ and Burst SCS $(B+D+F)$. Figure demonstrates a change of the BOLD signal response during SCS. The stimulus "on" period is shown by the dotted line.
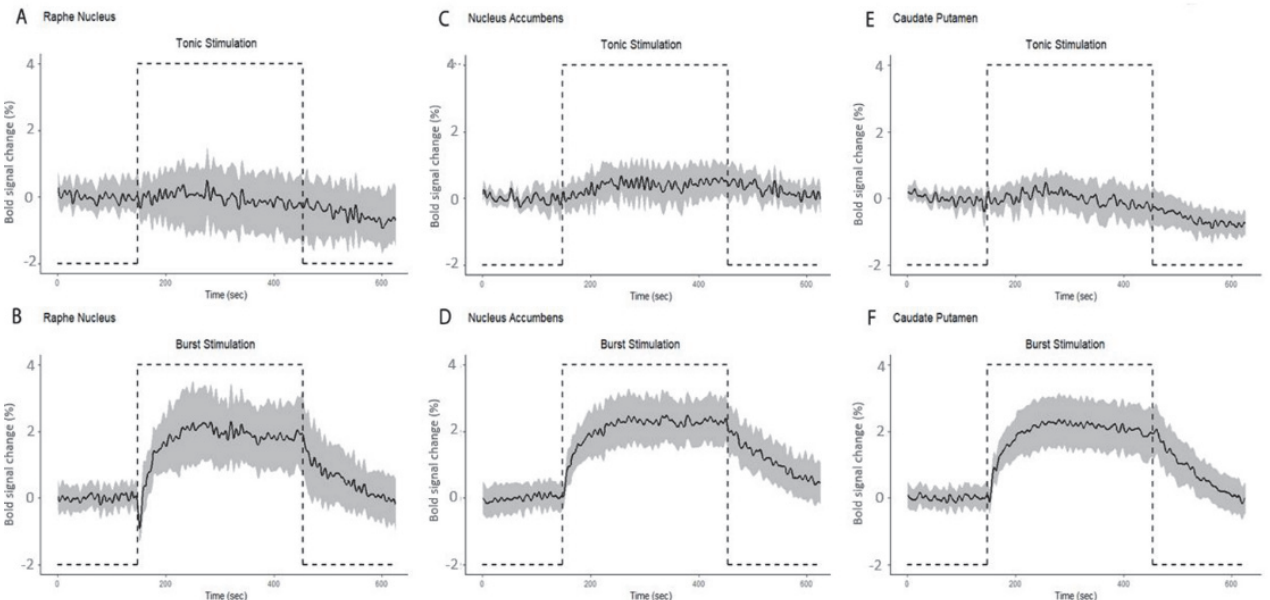

Fig.5: BOLD signal (\%). Average time-courses (mean \pm SEM) from representative brain regions associated with the processing of reward (nucleus accumbens and caudate putamen) and descending pain signals (raphe nuclei) for Tonic SCS $(A+C+E)$ and Burst SCS $(B+D+F)$. Figure demonstrates a change of the BOLD signal response during SCS. The stimulus "on" period is shown by the dotted line. 


\section{Bold Amplitude of Burst and Tonic SCS}

Analysis of Burst and Tonic SCS amplitude during stimulation showed that Burst SCS had, overall, a significantly stronger effect in the selected ROI's (Fig. 6). Two-Way ANOVA comparison of Burst pre-stimulation and Burst during stimulation showed that BOLD amplitude was significantly increased in the OFC, mPFC, ACCPFC, CPU, HIP, NucACC, Amy, Raphe, M1, M2, S1FL, S1HL, S2, as compared to pre-Burst stimulation (Table 2.) Two-Way ANOVA comparison of Tonic pre-stimulation and Tonic during stimulation showed that during stimulation showed that BOLD amplitude was significantly increased in the OFC, mPFC, ACCPFC, HIP, NuCACC, Amy, M1, M2, S1HL, S2, as compared to pre-Tonic stimulation. No significant activation differences were shown for the CPU, Raphe and S1FL (Table 2.).

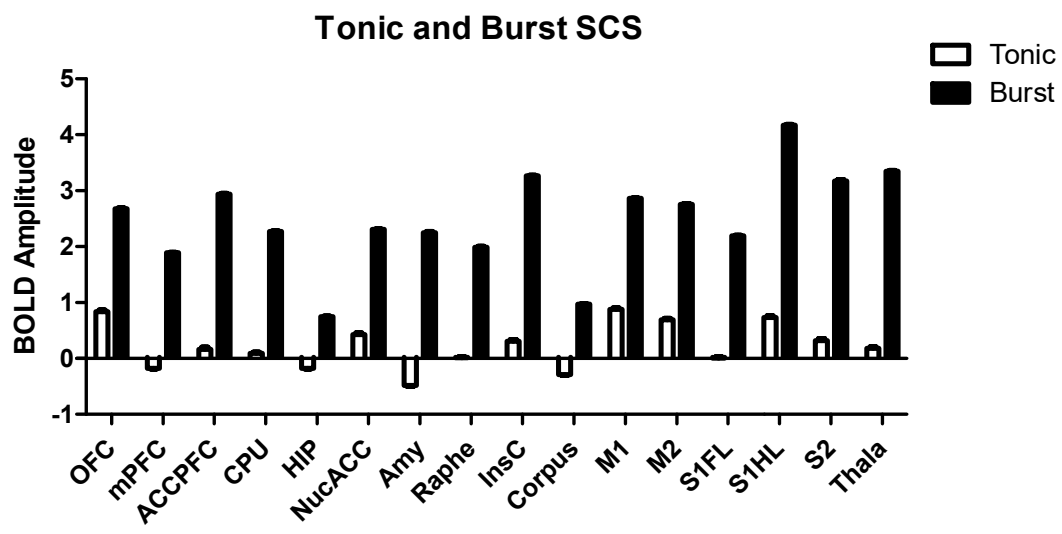

Fig.6: BOLD signal amplitude (\% change from baseline) in different ROIs during Burst and Tonic SCS. 


\begin{tabular}{|c|c|c|c|}
\hline \multicolumn{4}{|l|}{$\overline{\text { PRE VS. PLATEAU STIM }}$} \\
\hline & Difference (\%) & $P$ value & Significance \\
\hline ACCPFC & 0.2428 & $P<0.001$ & $* * *$ \\
\hline$C P U$ & 0.05059 & $P>0.05$ & ns \\
\hline HIP & -0.1950 & $P<0.001$ & $* * *$ \\
\hline NUCACC & 0.3931 & $P<0.001$ & $* * *$ \\
\hline$A M Y$ & -0.5487 & $P<0.001$ & $* * *$ \\
\hline RAPHE & -0.06078 & $P>0.05$ & ns \\
\hline INSC & 0.3323 & $P<0.001$ & $* * *$ \\
\hline$M 1$ & 0.9622 & $P<0.001$ & $* * *$ \\
\hline S1FL & 0.01319 & $P>0.05$ & ns \\
\hline S1HL & 0.7154 & $P<0.001$ & $* * *$ \\
\hline THALA & 0.1356 & $P<0.001$ & $* * *$ \\
\hline \multicolumn{4}{|l|}{ BURST SCS } \\
\hline \multicolumn{4}{|l|}{ PRE VS. PLATEAU STIM } \\
\hline & Difference (\%) & $P$ value & Significance \\
\hline ACCPFC & 2.818 & $P<0.001$ & $* * *$ \\
\hline$C P U$ & 2.199 & $P<0.001$ & $* * *$ \\
\hline HIP & 0.7168 & $P<0.001$ & $* * *$ \\
\hline NUCACC & 2.286 & $P<0.001$ & $* * *$ \\
\hline$A M Y$ & 2.029 & $P<0.001$ & $* * *$ \\
\hline RAPHE & 2.011 & $P<0.001$ & $* * *$ \\
\hline INSC & 3.192 & $P<0.001$ & $* * *$ \\
\hline M1 & 2.143 & $P<0.001$ & $* * *$ \\
\hline S1FL & 2.195 & $P<0.001$ & $* * *$ \\
\hline S1HL & 4.064 & $P<0.001$ & $* * *$ \\
\hline THALA & 3.271 & $P<0.001$ & $* * *$ \\
\hline
\end{tabular}

Table 2: BOLD signal amplitude (stimulation plateau BOLD signal minus pre-stimulation BOLD signal, $\%$ of baseline) for Tonic SCS ( $n=8)$ and Burst SCS ( $n=9)$. 


\section{Discussion}

The objective of the present study was to assess whether both Tonic SCS- and Burst SCSinduced pain relief in chronic neuropathic animals would result in increased BOLD-signal in brain regions associated with location and intensity of pain, and cognitive-emotional aspects of pain. We found that, overall, both Tonic SCS- and Burst SCS increased BOLD-signal in brain regions associated with location and intensity of pain (somatosensory cortex and premotor cortex), and cognitive-emotional aspects of pain (amygdala, anterior cingulate cortex, insular cortex). However, Burst SCS induced a more profound BOLD-increase in brain areas associated with cognitive-emotional aspects of pain, as compared to Tonic SCS. Additionally, we found that Burst SCS had a more profound BOLD-effect in the raphe nuclei and brain regions associated with the reward-system such as the nucleus accumbens and the caudate putamen. This was not observed in animals that received Tonic SCS.

Past research has demonstrated that $\mathrm{fMRI}$ can be a powerful tool for understanding and mapping of brain areas involved in the pain relieving effect of SCS [27, 28, 32]. FMRI has contributed, along with other neuro-imaging tools, to a deeper understanding of the mechanisms underlying Tonic SCS. It has been shown that Tonic SCS increases BOLD-levels in the secondary somatosensory cortex, primary sensorimotor cortex and the insula, while decreased BOLD-signals were reported in the primary motor cortex and primary somatosensory cortex. [28]. In line with these findings, in our study, we also observed increased BOLD-levels in the secondary somatosensory cortex and the insula during Tonic SCS. However, we did not observe decreased BOLD-signal during SCS in the primary motor cortex and primary somatosensory cortex. Yet, because physicians usually decrease the paresthesia threshold, in consultation with the patient, to a comfortable level, the animals in our study could have experienced higher levels of paresthesia, which would result in increased BOLD-signals in the primary somatosensory cortex. A more recent fMRI-study, with twenty patients that received Tonic-SCS treatment for FBSS, reported decreased BOLDsignal in the insula, the medial thalamus and the rostral and caudal cingulate cortex [27]. Another important finding from their study was that lesser BOLD-changes in the rostral ACC, inferior olivary nucleus and the cerebellum seemed to mark direct pain relief due to shortterm Tonic SCS. This emphasizes the role of the cerebello-thalamo-cortical circuit as a key regulator for Tonic SCS-induced pain relief and implicating the modulation and regulation of averse and negative affect related to pain with Tonic SCS [27]. This is partially in line with the findings of Deogaonkar and colleagues (2015) who demonstrated that Tonic SCS reduced affective components of pain when providing optimal pain relief. They reported a 
decreased connection strength between somatosensory and limbic areas and increased connection strength between somatosensory and default mode network with Tonic SCS [32]. This led them to hypothesize that pain relief from Tonic SCS could be induced by reduction of negative emotional processing associated with pain, allowing somatosensory areas to become more integrated into default mode activity [32]. This seems to suggest, in line with the aforementioned study and our results, that pain relief from Tonic SCS may be reducing negative emotional aspects of pain by activation of areas associated with the medial STT, be it to a lesser extent as compared to Burst SCS. Yet, this notion was challenged by De Ridder et al., who pondered whether Tonic-SCS really influences the medial pain system [55]. In response to the work of Deogaonkar and colleagues (2015), it was argued that pain unpleasantness is not correlated to a functional connectivity change between somatosensory cortex and dorsal anterior cingulate cortex/limbic areas. Therefore, they considered it premature to conclude that tonic stimulation does indeed exert its effect on pain suppression by modulating the emotional processing of pain. It thus seems that more neuro-imaging work is required in order to reach a clear consensus on the supraspinal working mechanisms of SCS.

Recently, Burst SCS was introduced to the field of neuromodulation [33, 34]. It is hypothesized that the Burst provides a more complete and adequate attenuation of the pain-experience, as initial clinical findings suggest that Burst SCS activates both the lateral and the medial STT [33]. The lateral STT is responsible for the transmission of objective painaspects such as the intensity and location of the painful stimulus. This pathway projects via the thalamus to cortical areas such as the somatosensory cortex [30, 31]. At the same time, brain signals travel along medial STT projection neurons to engage the cingulate and insular cortices via connections in the brainstem (parabrachial nucleus) and amygdala; contributing to the affective component of the pain experience. Thus, additional activation of medial STT with Burst SCS would also improve cognitive-emotional pain-aspects of neuropathic patients $[33,35]$. In this study, we found that, overall, stronger BOLD differences were observed for Burst stimulation as compared to Tonic stimulation. We observed a significantly increased BOLD-signal in the Amygdala, a key region of the medial STT-system, during Burst SCS, as compared to pre-stimulation. Meanwhile, Tonic SCS did not induce a significantly increased BOLD-signal in the Amygdala, as compared to pre-stimulation. Furthermore, two additional key-regions of the medial pain network, the insula and the cingulate cortex, showed a more profound increase in BOLD-signal during Burst SCS, as compared to Tonic SCS. This suggests that Burst stimulation indeed activates areas associated with the medial STT, which is in line with the work from the Ridder and 
colleagues who showed with encephalogram analysis that Burst SCS normalized the pain supporting/suppressing balance in contrast to Tonic SCS mode by a greater effect on the dorsal Anterior Cingulate Cortex (ACC) [35]. Meanwhile, in our study, Tonic SCS induced an increase in BOLD-levels in the primary somatosensory cortex and the premotor cortex, which is in line with previous studies which suggest that Tonic SCS mainly has an effect on cortical areas associated with the lateral STT [28]. Additionally, in our study, Tonic SCS increased BOLD-signal in areas associated with the medial pain pathway, although to a lesser extent than is seen with Burst SCS. It thus seems that both stimulation designs modify brain activity differently. It is important to mention that this in in contrast to the von Frey data obtained in this study and past literature, where Burst SCS and Tonic SCS did not significantly differ in optimal pain relieving effects $[39,40]$. Yet, it is conceivable that reflexbased assessment methods such as the von Frey are not sensitive to supraspinal cognitiveemotional aspects of pain, as reflexes are known to be processed (initially) on a spinal level [56-58].

Additionally, we found that Burst-SCS also activated brain areas associated with the reward-system; the caudate putamen and the nucleus accumbens [59]. This is not surprising, as the reward circuit play a vital role in the motivational-affective experience of pain [60]. In fact, chronic neuropathic pain is an extremely complex experience that requires the recruitment of an intricate set of central nervous system components. DosSantos and colleagues have highlighted brain areas associated with reward circuitry that play a role in the processing of pain, including the nucleus accumbens (NAc), ventral tegmental area, and the medial prefrontal cortex [60]. A study with healthy subjects has shown that at the offset of painful heat stimuli (rewarding) there is a decrease in BOLD signal in the NAc, while at the onset (aversive) an increase of BOLD-signal can be observed [61]. It could be hypothesized that part of the additional analgesic properties reported with Burst-SCS, as compared to Tonic SCS, are related to the activation of reward/motivational circuitry, and thus a feeling of reward [61]. Interestingly, the caudate putamen and the nucleus accumbens are also associated with the experience of motivation, a psychological aspect know to be affected by (neuropathic) pain [62-64]. Both acute and chronic pain can have a significant impact on the experience of motivation. More specifically, several studies have demonstrated how anatomical and functional changes in these motivational brain circuits contribute to the transition from acute to chronic pain [62]. In addition, a recent meta-analysis has reported that anhedonia is significantly associated with a reduced neural response to reward anticipation in the caudate nucleus, putamen and the nucleus 
accumbens [65]. Furthermore, the modulation of motivational pain aspects is in line with a recent experimental behavioral pain study (Meuwissen et al. 2019). By use of an operant testing method for rats, it was demonstrated that Burst SCS modulated cognitivemotivational aspects of pain behavior to a greater extent than Tonic SCS. It could be hypothesized that the increased activation levels observed in the nucleus accumbens and caudate putamen with Burst SCS are responsible for the pleasant feeling often described by patients who receive Burst stimulation. In line with this, De Ridder and Vanneste described the following: "Even though pain is commonly considered an aversive signal, in specific contexts (usually erotic) pain can be perceived as pleasurable". With this they referred to a study which showed that when pain is perceived as pleasurable, the anti-nociceptive system is activated, including the nucleus accumbens, pregenual anterior cingulate cortex and ventromedial prefrontal cortex as well as the caudate nucleus $[35,66]$. Nevertheless, the precise role the cognitive-motivational system plays in the supraspinal mechanisms underlying Burst SCS should be confirmed by future neuroimaging studies that include the complete subset of regions associated with the reward/motivation system. In our study, Burst SCS also activated the raphe nuclei. With Tonic SCS we did not observe significant BOLD-changes in this region. The raphe nuclei are an important set of nuclei in pain modulation. They have a great number of 5-HT neurons, and the vast fiber connections to other parts of the central nervous system provide the morphological basis for its painmodulating function. The descending projections of the raphe nuclei, via the nucleus raphe magnus or directly, modulate the processing of noxious stimuli in the spinal dorsal horn [67]. Interestingly, the nucleus raphe magnus receives descending afferents from the central nucleus of the amygdala, a brain region where we observed a substantial amount of activation with Burst SCS [68]. With this study, we provide a framework for future (fMRI) research on the supraspinal elements involved in pain relief during SCS. A deeper understanding of the neural pathways implicated in the neurophysiology of SCS-induced pain-relief will likely result in the development of new and alternative modalities with improved efficacy [69]. 
Chapter 6

\section{Conclusion}

Burst and Tonic SCS-induced pain relief is accompanied with different effects on the intensity and localization of SCS-induced increase of BOLD-signal in the brain. From this, we concluded that Burst SCS-induced pain relief is due to activation of brain regions associated with both objective aspects of pain and with cognitive-emotional aspects of pain relief. Secondly, we were able to demonstrate that Tonic SCS also induced increases in BOLD-signal in the aforementioned areas, but to a lesser extent. Additionally, our data suggest that Burst SCS might have additional analgesic properties over Tonic SCS in treatment of chronic neuropathic pain via the activation of brain areas associated with the reward-system and the raphe nuclei. 


\section{References}

1. Linderoth, B. and R.D. Foreman, Conventional and Novel Spinal Stimulation Algorithms: Hypothetical Mechanisms of Action and Comments on Outcomes. Neuromodulation, 2017. 20(6): p. 525-533.

2. Sdrulla, A.D., Y. Guan, and S.N. Raja, Spinal Cord Stimulation: Clinical Efficacy and Potential Mechanisms. Pain Pract, 2018. 18(8): p. 1048-1067.

3. Kumar, K., M. Abbas, and S. Rizvi, The use of spinal cord stimulation in pain management. Pain Manag, 2012. 2(2): p. 125-34.

4. North, R.B., et al., Spinal cord stimulation versus repeated lumbosacral spine surgery for chronic pain: a randomized, controlled trial. Neurosurgery, 2005. 56(1): p. 98-106; discussion 106-7.

5. Kumar, K., et al., The effects of spinal cord stimulation in neuropathic pain are sustained: a 24month follow-up of the prospective randomized controlled multicenter trial of the effectiveness of spinal cord stimulation. Neurosurgery, 2008. 63(4): p. 762-70; discussion 770.

6. Kumar, K., et al., Spinal cord stimulation versus conventional medical management for neuropathic pain: a multicentre randomised controlled trial in patients with failed back surgery syndrome. Pain, 2007. 132(1-2): p. 179-88.

7. Visnjevac, O., et al., A Comprehensive Outcome-Specific Review of the Use of Spinal Cord Stimulation for Complex Regional Pain Syndrome. Pain Pract, 2017. 17(4): p. 533-545.

8. Taylor, R.S., J.P. Van Buyten, and E. Buchser, Spinal cord stimulation for complex regional pain syndrome: a systematic review of the clinical and cost-effectiveness literature and assessment of prognostic factors. Eur J Pain, 2006. 10(2): p. 91-101.

9. Verrills, P., C. Sinclair, and A. Barnard, A review of spinal cord stimulation systems for chronic pain. J Pain Res, 2016. 9: p. 481-92.

10. Rodriguez, M.J., et al., Invasive Management for Pediatric Complex Regional Pain Syndrome: Literature Review of Evidence. Pain Physician, 2015. 18(6): p. 621-30.

11. Rho, R.H., et al., Complex regional pain syndrome. Mayo Clin Proc, 2002. 77(2): p. 174-80.

12. Reig, E. and D. Abejon, Spinal cord stimulation: a 20-year retrospective analysis in 260 patients. Neuromodulation, 2009. 12(3): p. 232-9.

13. Poree, L., et al., Spinal cord stimulation as treatment for complex regional pain syndrome should be considered earlier than last resort therapy. Neuromodulation, 2013. 16(2): p. 12541.

14. Health Quality, O., Spinal cord stimulation for neuropathic pain: an evidence-based analysis. Ont Health Technol Assess Ser, 2005. 5(4): p. 1-78.

15. Goebel, A., Current Concepts in Adult CRPS. Rev Pain, 2011. 5(2): p. 3-11.

16. Kemler, M.A., et al., The cost-effectiveness of spinal cord stimulation for complex regional pain syndrome. Value Health, 2010. 13(6): p. 735-42.

17. van Beek, M., et al., Sustained Treatment Effect of Spinal Cord Stimulation in Painful Diabetic Peripheral Neuropathy: 24-Month Follow-up of a Prospective Two-Center Randomized Controlled Trial. Diabetes Care, 2015. 38(9): p. e132-4.

18. Slangen, R., et al., Spinal cord stimulation and pain relief in painful diabetic peripheral neuropathy: a prospective two-center randomized controlled trial. Diabetes Care, 2014. 37(11): p. 3016-24.

19. de Vos, C.C., et al., Spinal cord stimulation in patients with painful diabetic neuropathy: a multicentre randomized clinical trial. Pain, 2014. 155(11): p. 2426-31.

20. Yang, F., et al., Comparison of intensity-dependent inhibition of spinal wide-dynamic range neurons by dorsal column and peripheral nerve stimulation in a rat model of neuropathic pain. Eur J Pain, 2014. 18(7): p. 978-88. 
21. Shechter, R., et al., Conventional and kilohertz-frequency spinal cord stimulation produces intensity-and frequency-dependent inhibition of mechanical hypersensitivity in a rat model of neuropathic pain. Anesthesiology, 2013. 119(2): p. 422-32.

22. Sdrulla, A.D., et al., Electrical stimulation of low-threshold afferent fibers induces a prolonged synaptic depression in lamina II dorsal horn neurons to high-threshold afferent inputs in mice. Pain, 2015. 156(6): p. 1008-17.

23. Janssen, S.P., et al., Decreased intracellular GABA levels contribute to spinal cord stimulationinduced analgesia in rats suffering from painful peripheral neuropathy: the role of KCC2 and GABA(A) receptor-mediated inhibition. Neurochem Int, 2012. 60(1): p. 21-30.

24. Cui, J.G., et al., Effect of spinal cord stimulation on tactile hypersensitivity in mononeuropathic rats is potentiated by simultaneous $G A B A(B)$ and adenosine receptor activation. Neurosci Lett, 1998. 247(2-3): p. 183-6.

25. Cui, J.G., B. Linderoth, and B.A. Meyerson, Effects of spinal cord stimulation on touch-evoked allodynia involve GABAergic mechanisms. An experimental study in the mononeuropathic rat. Pain, 1996. 66(2-3): p. 287-95.

26. Smits, H., et al., Experimental spinal cord stimulation and neuropathic pain: mechanism of action, technical aspects, and effectiveness. Pain Pract, 2013. 13(2): p. 154-68.

27. Moens, M., et al., Spinal cord stimulation modulates cerebral function: an fMRI study. Neuroradiology, 2012. 54(12): p. 1399-407.

28. Stancak, A., et al., Functional magnetic resonance imaging of cerebral activation during spinal cord stimulation in failed back surgery syndrome patients. Eur J Pain, 2008. 12(2): p. 137-48.

29. Kiriakopoulos, E.T., et al., Functional magnetic resonance imaging: a potential tool for the evaluation of spinal cord stimulation: technical case report. Neurosurgery, 1997. 41(2): p. 5014.

30. Kulkarni, B., et al., Attention to pain localization and unpleasantness discriminates the functions of the medial and lateral pain systems. Eur J Neurosci, 2005. 21(11): p. 3133-42.

31. Basbaum, A.I., et al., Cellular and molecular mechanisms of pain. Cell, 2009. 139(2): p. 267-84.

32. Deogaonkar, M., et al., Spinal Cord Stimulation (SCS) and Functional Magnetic Resonance Imaging (fMRI): Modulation of Cortical Connectivity With Therapeutic SCS. Neuromodulation, 2016. 19(2): p. 142-53.

33. De Ridder, D., et al., Burst spinal cord stimulation for limb and back pain. World Neurosurg, 2013. 80(5): p. 642-649 e1.

34. De Ridder, D., et al., Burst spinal cord stimulation: toward paresthesia-free pain suppression. Neurosurgery, 2010. 66(5): p. 986-90.

35. De Ridder, D. and S. Vanneste, Burst and Tonic Spinal Cord Stimulation: Different and Common Brain Mechanisms. Neuromodulation, 2016. 19(1): p. 47-59.

36. Seltzer, Z., R. Dubner, and Y. Shir, A novel behavioral model of neuropathic pain disorders produced in rats by partial sciatic nerve injury. Pain, 1990. 43(2): p. 205-18.

37. Truin, M., et al., Successful pain relief in non-responders to spinal cord stimulation: The combined use of ketamine and spinal cord stimulation. European Journal of Pain, 2011. 15(10).

38. Truin, M., et al., Increased efficacy of early spinal cord stimulation in an animal model of neuropathic pain. Eur J Pain, 2011. 15(2): p. 111-7.

39. Meuwissen, K.P.V., et al., Burst Spinal Cord Stimulation in Peripherally Injured Chronic Neuropathic Rats: A Delayed Effect. Pain Pract, 2018.

40. Meuwissen, K.P.V., et al., Conventional-SCS vs. Burst-SCS and the Behavioral Effect on Mechanical Hypersensitivity in a Rat Model of Chronic Neuropathic Pain: Effect of Amplitude. Neuromodulation, 2018. 21(1): p. 19-30.

41. Chaplan, S.R., et al., Quantitative assessment of tactile allodynia in the rat paw. J Neurosci Methods, 1994. 53(1): p. 55-63. 
42. Smits, $\mathrm{H}$., et al., Effect of spinal cord stimulation in an animal model of neuropathic pain relates to degree of tactile "allodynia". Neuroscience, 2006. 143(2): p. 541-6.

43. Smits, H., M. van Kleef, and E.A. Joosten, Spinal cord stimulation of dorsal columns in a rat model of neuropathic pain: evidence for a segmental spinal mechanism of pain relief. Pain, 2012. 153(1): p. 177-83.

44. Cui, J.G., et al., Spinal cord stimulation attenuates augmented dorsal horn release of excitatory amino acids in mononeuropathy via a GABAergic mechanism. Pain, 1997. 73(1): p. 87-95.

45. Jenkinson, M., et al., Improved optimization for the robust and accurate linear registration and motion correction of brain images. Neuroimage, 2002. 17(2): p. 825-41.

46. Sled, J.G., A.P. Zijdenbos, and A.C. Evans, A nonparametric method for automatic correction of intensity nonuniformity in MRI data. IEEE Trans Med Imaging, 1998. 17(1): p. 87-97.

47. Smith, S.M., Fast robust automated brain extraction. Hum Brain Mapp, 2002. 17(3): p. 143-55.

48. Jenkinson, M. and S. Smith, A global optimisation method for robust affine registration of brain images. Med Image Anal, 2001. 5(2): p. 143-56.

49. Andersson, P., et al., $\mathrm{BCl}$ control using 4 direction spatial visual attention and real-time fMRI at 7T. Conf Proc IEEE Eng Med Biol Soc, 2010. 2010: p. 4221-5.

50. Jenkinson, M., et al., Fsl. Neuroimage, 2012. 62(2): p. 782-90.

51. Paxinos, G., Watson, C., The rat brain in stereotaxic coordinates. Academic Press, New York, 2007. 6th edition.

52. Karel, P., et al., Ultrahigh-resolution MRI reveals structural brain differences in serotonin transporter knockout rats after sucrose and cocaine self-administration. Addict Biol, 2019.

53. Sarabdjitsingh, R.A., et al., Early life stress-induced alterations in rat brain structures measured with high resolution MRI. PLoS One, 2017. 12(9): p. e0185061.

54. Mills, C., et al., Estimating efficacy and drug ED50's using von Frey thresholds: impact of weber's law and log transformation. J Pain, 2012. 13(6): p. 519-23.

55. De Ridder, D. and S. Vanneste, Does Tonic Spinal Cord Stimulation Really Influence the Medial Pain System? Neuromodulation, 2016. 19(2): p. 227-8.

56. Matthies, B.K. and K.B. Franklin, Formalin pain is expressed in decerebrate rats but not attenuated by morphine. Pain, 1992. 51(2): p. 199-206.

57. Mogil, J.S., Animal models of pain: progress and challenges. Nat Rev Neurosci, 2009. 10(4): p. 283-94.

58. Woolf, C.J., Long term alterations in the excitability of the flexion reflex produced by peripheral tissue injury in the chronic decerebrate rat. Pain, 1984. 18(4): p. 325-43.

59. Berridge, K.C. and M.L. Kringelbach, Pleasure systems in the brain. Neuron, 2015. 86(3): p. 64664.

60. DosSantos, M.F., B.S. Moura, and A.F. DaSilva, Reward Circuitry Plasticity in Pain Perception and Modulation. Front Pharmacol, 2017. 8: p. 790.

61. Becerra, L. and D. Borsook, Signal valence in the nucleus accumbens to pain onset and offset. Eur J Pain, 2008. 12(7): p. 866-9.

62. Porreca, F. and E. Navratilova, Reward, motivation, and emotion of pain and its relief. Pain, 2017. 158 Suppl 1: p. S43-S49.

63. Borsook, D., et al., Reward deficiency and anti-reward in pain chronification. Neurosci Biobehav Rev, 2016. 68: p. 282-297.

64. Becker, S. and M. Diers, [Chronic pain : Perception, reward and neural processing]. Schmerz, 2016. 30(5): p. 395-406.

65. Zhang, B., et al., Mapping anhedonia-specific dysfunction in a transdiagnostic approach: an ALE meta-analysis. Brain Imaging Behav, 2016. 10(3): p. 920-39.

66. Leknes, S., et al., The importance of context: when relative relief renders pain pleasant. Pain, 2013. 154(3): p. 402-10. 
Chapter 6

67. Wang, Q.P. and Y. Nakai, The dorsal raphe: an important nucleus in pain modulation. Brain Res Bull, 1994. 34(6): p. 575-85.

68. Hermann, D.M., et al., Afferent projections to the rat nuclei raphe magnus, raphe pallidus and reticularis gigantocellularis pars alpha demonstrated by iontophoretic application of choleratoxin (subunit b). J Chem Neuroanat, 1997. 13(1): p. 1-21.

69. Flor, H., New developments in the understanding and management of persistent pain. Curr Opin Psychiatry, 2012. 25(2): p. 109-13. 


\title{
CHAPTER 7
}

\section{SUMMARY, DISCUSSION, AND CONCLUSION}

\author{
Aims and Research questions
}

The primary aim of this academic thesis was to elucidate the spinal and supraspinal mechanisms underlying Burst spinal cord stimulation induced pain relief in an experimental neuropathic pain model based on the understanding of the underlying

mechanism.

RESEARCH QUESTIONS (RQ)

Based on the aims of this academic thesis, the following research questions are formulated:

1. What is the effect of amplitude on the analgesic efficacy of Burst- and Tonic SCS in an experimental animal model of chronic neuropathic pain?

2. Does the anti-nociceptive effect over time differ for Burst SCS and Tonic SCS?

3. Is GABA involved in the spinal pain relieving mechanism of Burst SCS?

4. Is it possible to discriminate between Burst and Tonic SCS on cognitive-motivational aspects of pain relief, with use of MCAS operant testing?

5. Which brain areas are involved in the pain relieving effect of Burst SCS, as compared to Tonic SCS, in an animal model of chronic neuropathic pain?

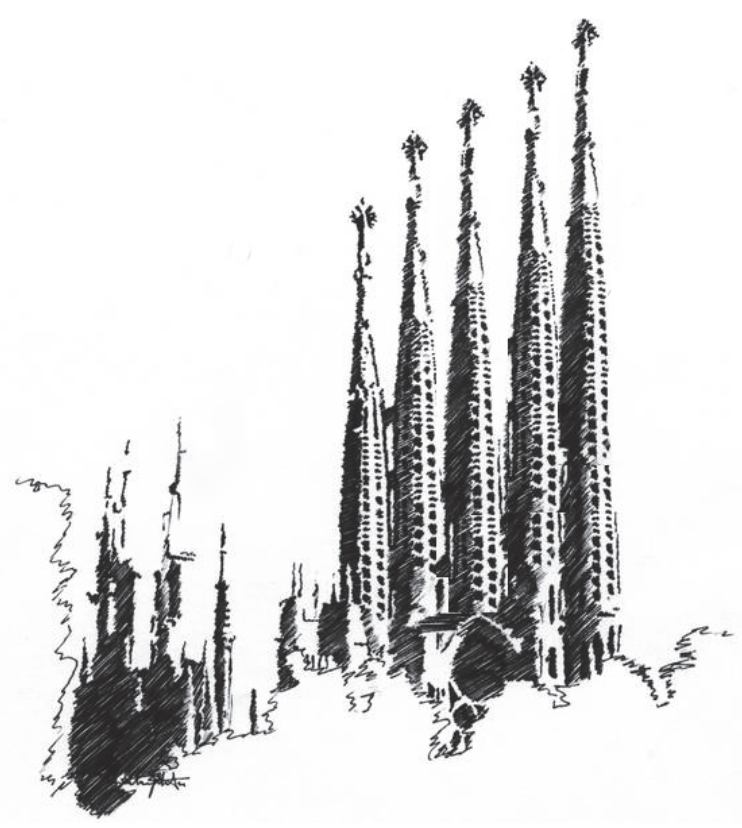


The conclusions that have been formulated based on the experiments as described in the various chapters will be discussed in broader and future perspectives and related to the aims of this thesis.

The first research question formulated was:

\section{What is the effect of amplitude on the analgesic efficacy of Burst- and Tonic SCS in an experimental animal model of chronic neuropathic pain?}

To answer this question, we assessed mechanical hypersensitivity in 12 Sprague Dawley rats using paw withdrawal thresholds (WTs) to von Frey monofilaments, at various SCS intensities (amplitudes) and multiple time points during 60 minutes of stimulation and 30 minutes post stimulation. We found that Burst SCS requires significantly more charge per second (CPS) in order to achieve similar pain relief compared to Tonic SCS, in an experimental model of chronic neuropathic pain. Increasing amplitude with Tonic SCS improved its efficacy, whereas the efficacy of Burst SCS showed a non-monotonic relation with amplitude. Interestingly, Tonic SCS at 66\% MT and Burst SCS at 50\% MT had a statistically similar maximum pain relieving effect, assessed as decrease in mechanical hypersensitivity. However, Burst SCS required higher mean CPS in order to achieve this maximum pain relieving effect. Moreover, when Burst SCS and Tonic SCS were applied at a comparable mean CPS Tonic SCS had a superior behavioral outcome. Our findings further demonstrate that Burst- and Tonic SCS exhibit an essentially different behavioral pattern of pain relief, in relation to amplitude (at $66 \%, 50 \%$ and $33 \% \mathrm{MT}$ ). For Tonic SCS, amplitude is positively correlated with behavioral outcome, as an increase in amplitude is accompanied by an increase in pain relief. Contrarily, Burst SCS amplitude does not appear to have a positive correlation with the behavioral outcome, as 66\% MT, the highest applied amplitude, did not produce optimal pain relief. This implies a non-monotonic relation with amplitude. Furthermore, the range of amplitudes for Burst SCS in order to achieve an optimal behavioral effect (therapeutic window) appeared to be relatively narrow given that the behavioral outcome was shown to decline rather rapidly once $50 \% \mathrm{MT}$ (= optimal Burst MT) is surpassed. This is consistent with the findings of Courtney and colleagues who reported that the therapeutic range of Burst SCS is considerably smaller (perception amplitude $0.3 \mathrm{~mA}$, max tolerable amplitude $2.5 \mathrm{~mA}$ ) compared to Tonic SCS (perception amplitude $4.2 \mathrm{~mA}$, max tolerable amplitude $8.9 \mathrm{~mA}$ ) [1]. However, we cannot exclude the assumption that a gradual decrease of the behavioral outcome is enclosed within the two highest amplitudes applied with Burst SCS in our study. Thus, Burst SCS is effective at smaller 
amplitudes, yet the overall CPS necessary to obtain a therapeutic effect is significantly higher when compared to Tonic SCS. Remarkably, the large quantities of charge delivered with Burst SCS do not result in a superior behavioral outcome, compared with Tonic SCS (the maximum WT of the optimal Burst amplitude (50\%) and Conventional amplitude (66\%) did not significantly differ). This is in line with recent clinical findings from which it was concluded that stimulation with Tonic SCS and Burst SCS are equally effective in relieving neuropathic pain related to complex regional pain syndrome [2]. It is, therefore, reasonable to assume that both Tonic- and Burst SCS modes are able to induce significant pain relief; despite there being significant differences in CPS necessary for similar pain relief. With the recent focus on new frequencies and waveforms in the SCS field, there is a growing appreciation for the impact of energy delivery on the central nervous system [3]. A recent clinical study suggested a possible dose-related response between the amount of energy delivered to the spinal cord and the clinical effect [4]. Understanding the physiological consequences of delivering energy to the spinal cord could aid the optimization process of (Burst) SCS and therefore deserves more future research.

When all parameters are considered together, stimulation is characterized by "charge over time," which can be delivered through various dosing strategies (Burst SCS, Tonic SCS, HF-SCS). For example, Tonic SCS "doses" therapy with a high "charge per pulse." In contrast, a dosing strategy that uses higher frequencies and/or wider pulse widths produces a more consistent delivery of energy, providing a higher CPS. Thus, stimulation parameters can be characterized as having a low or high concentration of current, and the delivery of energy could be characterized as involving low or high dose. The basic unit of electrical stimulation in neuromodulation is the pulse. The pulse consists of a specific amount of current amplitude (measured in milliAmperes, $\mathrm{mA}$ ) for a specific amount of time (pulse width). Each pulse is followed by an equal flow of current in the opposite direction in order to balance the charge delivered to the spinal cord and thereby safeguard against overstimulation (buildup of charge) which is known to result in tissue damage [5]. The "recharge" phase can be active (producing a biphasic appearance) or passive (balanced but asymmetric); in most SCS systems, charge balancing occurs after each individual pulse and in a passive manner [3]. With the introduction of the Burst waveform however, the passive recharge balancing should not be taken for granted. The Burst waveform as it was introduced to the SCS-field by Dr. De Ridder, also known as the BurstDR waveform is a passive-recharge form of Burst SCS (as opposed to an active recharge waveform applied in our studies presented in this thesis), where the built-up charge period is discharged during the interburst-period $[6,7]$. In our studies, we applied biphasic Burst waveform, that discharges in an active recharge 
fashion, during the intraburst-period, in order to maintain the charge balance. It is proposed that this difference between active and passive Burst SCS can have physiological repercussions: the 'plateau' that occurs during the 'active phase', followed by a period of relative quiescence called the "silent phase'" with BurstDR-SCS is generated by calcium influx via T-type calcium channels, generating a low threshold $\mathrm{Ca} 2+$ potential upon the crest of which sodium and potassium channel-mediated action potentials fire, creating low threshold bursts. This is hypothesized to mimic 'natural' burst firing [8-10]. However, while the burst waveform used in our study has a different shape from the one utilized in other studies, physiological or clinical differences between these burst waveforms are unknown. To date, no data from clinical or preclinical studies support the claim that passive recharge burst is more effective than active recharge burst, and future studies aiming to directly compare active and passive recharge Burst waveforms should be conducted in order to shed light upon this matter. In order to more accurately interpret the effect specific parameter variations can have on energy delivery to the spinal cord one should consult the "strengthduration curve" as presented in the review by Miller and colleagues [3]. The strengthduration curve demonstrates that short pulse widths require high amplitudes to activate a neuron or axon, while wider pulse widths require less amplitude. In vivo, each individual axon has its own strength-duration curve based on its size, grade of myelination, and distance from the locus of stimulation. The concept of the strength-duration curve has been demonstrated in studies testing activation of spinal cord axons in animal models [11], and in patients implanted with SCS systems $[12,13]$. Generally, amplitude impacts the number of fibers recruited in the spinal cord, and results in a perceived increase or decrease in the intensity and/or area of paresthesia sensation. On the other hand, pulse width is typically considered a secondary factor in controlling energy delivery, however, an increase in this parameter can also recruit a larger number of fibers, and decreases the need for amplitude. An understanding of the strength-duration curve also elucidates how a substantial amount of current can be delivered to an axon without necessarily producing an action potential. When the current delivered is below the activation threshold for a neuron, the neuron may locally depolarize, but no action potential is generated. Furthermore, large amounts of charge could theoretically be administered at extreme ends of the curve (with narrow or wide pulse width), while still remaining below threshold and avoiding the development of intense paresthesia [3]. Therefore, Burst or HF stimulation may be subthreshold with respect to neuronal activation, and sub-perception with respect to the patient's experience, yet large amounts of charge is delivered to the spinal cord and thus can manipulate spinal neural functioning. Altogether, the findings presented in Chapter 2 of this thesis suggest 
that a more complex relationship defines the interplay between the parameters of SCS, the charge delivered to the spinal cord, and the therapeutic efficacy of SCS. With our study, where we investigated the effect of amplitude variation, we have presented a framework for future parameter variation studies. We believe that greater insight into the various parameter combinations will help to optimize (Burst) SCS.

\section{Research question 2: Does the anti-nociceptive effect over time differ for Burst SCS and Tonic SCS?}

To answer the second research question 43 neuropathic animals received either Burst or Tonic SCS for 60 minutes, while undergoing behavioral von Frey assessment during 60 minutes of stimulation and 30 minutes post stimulation. We reported a delayed antinociceptive effect of Burst SCS after onset of the stimulation, as compared with Tonic SCS. Furthermore, Burst SCS seemed to exhibit a delayed washout of analgesia after stimulation was turned off. However, no difference in maximum anti-nociceptive effect of Burst SCS, as compared with Tonic SCS, was noted in our study.

t has previously been suggested that Burst SCS provides a more complete and adequate attenuation of the pain-experience, as initial clinical findings suggest that Burst SCS activates both the lateral and the medial STT [6]. This is in line with our findings presented in Chapter 6, where we described that Burst SCS induced a more profound BOLD-increase in brain areas overall, and in brain areas associated with cognitive-emotional aspects of pain, as compared to Tonic SCS. These results corroborate the prevailing hypothesis that Burst SCS stimulates supraspinal pathways able to activate brain areas associated with cognitive-motivational aspects of pain $[6,14]$. Modulation of cognitive-motivational aspects of pain with Burst SCS is also in line with our findings presented in Chapter 5, where we discussed the outcome of our behavioral study performed with use of the Mechanical Conflict Avoidance System (MCAS). With the MCAS-system, we studied operant behavior specifically correlated with cognitive-motivational aspects of pain. From this, we concluded that Burst SCS specifically affects, much more than Tonic SCS, the processing of cognitivemotivational aspects of pain. On the other hand, the main mechanism of action underlying the analgesic effect of Tonic SCS has been shown to act through antidromic activation of $A \beta$-fibers at a segmental, spinal, level $[15,16]$. Supraspinal processes, set in motion by orthodromic activation of A $\beta$-fibers with Tonic SCS, eventually lead to secondary analgesic aspects of Tonic SCS [17]. Following this line of reasoning, it could be hypothesized that activation of supraspinal pathways, as suggested with Burst SCS, requires a different 
timeframe in order to fully establish its anti-nociceptive properties, as compared to segmental mechanisms observed with Tonic SCS. Thus, the anatomical location of the main mechanisms of action might underlie the delayed onset and timing difference between the behavioral effects of Burst SCS and Tonic SCS as observed in our study (Chapter 3). Furthermore, Burst SCS has been shown to modulate attention to pain, as shown by studies that utilized the Pain Vigilance and Awareness questionnaire [6]. It is conceivable that such an attention-modulation effect, mediated by brain structures associated with the limbic system, reverberates after cessation of stimulation, as compared to direct modulation of segmental mechanisms at a spinal level. Therefore, one could hypothesize that the attention-modulating effect of Burst SCS might echo on after the stimulation is terminated, which is reflected by a delayed washout effect, as compared with Tonic SCS. Computer modelling has indicated that SCS frequency, at least partly, determines the firing of Wide Dynamic Range (WDR) neurons projecting to the brain [18]. It was demonstrated that SCS with frequencies above $100 \mathrm{~Hz}$ (as with Burst SCS) do not result in inhibition of WDR signaling; this strongly suggests that higher SCS frequencies induce pain relief via alternative mechanisms. If future research demonstrates that certain parameters are indeed involved with the modulation of specific physiological components, SCS-modes could be tailormade' for each patient, which would result in optimal pain relief for each individual patient through specific targeting of the underlying pathology. Yet, future experiments should be performed in order to shed more light on the differences in pathway activation associated with different waveforms.

\section{Research question 3: Is GABA involved in the spinal pain relieving mechanism of Burst SCS?}

For the third RQ, which was addressed in Chapter 4, experiments were performed in a widely recognized rat model for SCS and chronic neuropathic pain: partial sciatic nerve ligation (PSNL) model [19].

In our study, anatomical analysis based on detailed quantitative immunohistochemistry of spinal dorsal horn GABA revealed that 60 minutes of Tonic SCS induced a decrease (trend) of intracellular GABA-levels. This is in line with past research performed in the PSNL-model $[20,21]$. During the development of neuropathic pain Janssen and colleagues observed, in the partial sciatic nerve ligation model, increased intracellular levels of GABA in the dorsal horn [20]. In a follow-up study they then showed that Tonic SCS decreased intracellular GABA-immunoreactivity in the dorsal horn of these PSNL-rats [21]. Quantitative 
immunohistochemistry of spinal dorsal horn GABA-levels in our study further revealed a significant decrease in intracellular GABA levels after 60 minutes of Burst SCS. Intracellular GABA levels were significantly decreased compared to Sham-SCS animals and Tonic SCS animals. Intrathecal administration of the $\mathrm{GABA}_{\mathrm{A}^{-}}$and $\mathrm{GABA}_{\mathrm{B}}-$ receptor antagonist bicuculline and phaclofen revealed that blocking the spinal GABAergic mechanisms abolishes the pain relieving effect of both Tonic SCS and Burst SCS. Based on these findings, we concluded in Chapter 4 that the pain relieving effect of Burst SCS includes a spinal GABAergic mechanism. In line with our study, preclinical studies, conducted in a sciatic nerve injury model, demonstrated that intrathecal administration of the $G A B A_{B}$-agonist baclofen, and the $\mathrm{GABA}_{\mathrm{A}}$-agonist muscimol, administered intrathecally, resulted in a marked and long-lasting increase of withdrawal thresholds in rats not receiving adequate pain relief with Tonic SCS (non-responders), although muscimol produced a less prominent threshold increase as compared to baclofen [48]. This, and our data, shows that Tonic SCS operates by potentiating the spinal GABAergic systems in general, and that the Tonic SCS effect depends on both $G_{A B A_{A}}$-receptor and $G A B A_{B}$-receptor signaling, although the SCS-

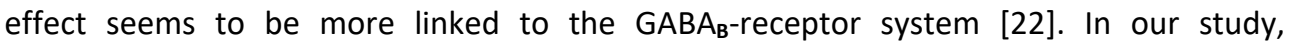
administration of a $G A B A_{A}$ - and $G A B A_{B}$-receptor antagonist also abolished the pain relieving effect of Burst SCS. Our observations, which led us to conclude that Burst SCS is mediated via spinal GABAergic mechanisms, seem not to be in line with findings reported by Crosby and colleagues (2015). Based on electrophysiological analysis of neuronal firing after Burst $\mathrm{SCS}$, these authors reported that the presence of a $\mathrm{GABA}_{B}$-receptor antagonist did not block the attenuation of dorsal horn neuronal firing, when SCS was applied at $90 \%$ of the Motor Threshold (MT) [23]. Yet, because they made use of a rather unconventional rat model of pain, and because they applied SCS at 90\% MT (an intensity known to induce unwanted side effects in the animals), it remains difficult to compare their findings to the bulk of experimental SCS studies performed with use of peripheral nerve injury models $[8,19,21$, 24-28], and SCS applied below 90\% MT.

Since Burst SCS is known to modulate structures at a supraspinal level in a different manner as compared to Tonic SCS $[6,14]$, it is remarkable that the segmental GABAergic mechanisms of these waveforms, at the spinal level, show similarities. Evidence has shown that Tonic SCS, via descending pathways, exerts an inhibitory GABAergic effect in the dorsal horn of the spinal cord [29-34]. It thus might be possible that Burst SCS via similar descending pathways is able to release GABA in the spinal dorsal horn. Taking into consideration the findings described in Chapter 3, where we observed a delayed onset for the analgesic effect of Burst SCS, the onset of GABAergic mechanisms activated by Burst 
SCS, if induced by the supraspinal loop, could be responsible for the delayed effect. On the other hand, according to existing literature it could be hypothesized that the mechanism of Burst SCS might include antidromic actions that lead to direct release of GABA in the spinal dorsal horn. Initially, the lack of paresthesia as observed with Burst and HF SCS brought forward the notion that these waveforms do not activate $A \beta$-fibers, neither orthodromically nor antidromically. Yet, $A \beta$-fibers are thickly myelinated, and thus very susceptible for depolarization with any form of SCS [35, 36]. Furthermore, Burst SCS is known to deliver a high charge per second to the spinal cord, and studies have reported that (naturally occurring) bursts have a higher probability to generate postsynaptic potentials, as compared to single tonic action potentials (which activate $A \beta$-fibers) [37]. Therefore, it is not inconceivable that $A \beta$-fibers absorb a great amount of the charge delivered with Burst SCS, which could potentially lead to subthreshold activation of said fibers [3]. Subthreshold $A \beta$-fiber activation might be insufficient for the patient to perceive paresthesia with Burst SCS, however, it might be sufficient enough for the release of GABA in the dorsal horn via antidromic activation of inhibitory GABAergic interneurons [16].

The majority of experimental studies on the effect of Tonic SCS were performed in sciatic nerve injury models including the sciatic nerve ligation model (PSNL) and the chronic constriction injury model [8, 15, 21, 22, 24, 27, 28, 38-40]. Therefore, in order to adequately compare and correlate our findings to previous literature, we deliberately chose to perform our experiments in the partial sciatic nerve ligation (PSNL) model. Animal models are pivotal for understanding the mechanism of NPP and for the development of effective analgesic therapies. Over the years, a battery of NPP models has been developed to simulate clinical NPP conditions with diverse etiology. Use of these models has contributed immensely to the understanding of NPP and underlying pathogenic mechanisms [41]. An example is the experimental study performed by Cui and colleagues who, in a sciatic nerve lesion model, demonstrated that rats not receiving adequate pain relief with Tonic SCS (non-responders) could be turned into responders via administration of sub-effective doses of the $G_{A B A}$ receptor agonist baclofen [24]. The aforementioned preclinical finding, the synergistic effect between Tonic SCS and baclofen application in PSNL rats, was successfully translated to the clinic where neuropathic pain patients, not responding adequately to Tonic SCS, showed improved pain relief following the intrathecal administration of baclofen [42]. Yet, despite the aforementioned example, progress in the field of translational pain research remains very limited [43]. Novel insights and analgesic treatments successful in experimental setting, acquired via basic science and animal models for NPP, have not led to equal development of novel clinically effective compounds. A careful re-examination of 
animal models of pain is therefore warranted, and it is all the more important that there will be consistency and a consensus on the animal models used to investigate specific areas of pain research including SCS for the treatment of NPP.

\section{Research question 4: Is it possible to discriminate between Burst and Tonic SCS on cognitive-motivational aspects of pain relief, with use of MCAS operant testing?}

To answer this RQ, which was described in Chapter 5, a novel Mechanical Conflict Avoidance System (MCAS) was used to study operant behavior specifically correlated with cognitivemotivational aspects of pain.

Escape latency on the MCAS operant testing setup was evaluated at various probe heights for rats $(n=26)$ with chronic neuropathic pain induced by a partial sciatic nerve ligation (PSNL). From this, we concluded that the MCAS is a valid and reproducible method for the assessment of SCS-induced cognitive-motivational behavioral aspects of pain relief. Use of the MCAS operant testing method revealed significant differences in cognitivemotivational behavior for Burst SCS and Tonic SCS, and this difference could not be detected with reflex-based Von Frey testing. Furthermore, our behavioral findings strongly suggested that Burst SCS specifically affects, much more than Tonic SCS, the processing of cognitivemotivational aspects of pain. Our experimental data are in line with, and partly confirm, observations with Burst SCS in the clinical setting, where questionnaires regarding attention to pain and affective aspects of pain (PVAQ) and patients' preference all tend to lean towards Burst SCS, as compared to other SCS-waveforms [44-46]. Nevertheless, till today, there is still no clear consensus in literature, neither preclinical $[8,28,47]$ nor clinical $[6,7$, $14,44-46,48-53$ ], regarding the objective analgesic efficacy of Burst SCS (as measured on the VAS-scale), as compared to other SCS-waveforms. Interestingly, a recent clinical study by Kriek and colleagues has shown that the preferred stimulation setting is not solely driven by the amount of pain reduction, but is also influenced by which stimulation setting feels most comfortable [51]. Therefore, it is vital that we aim to elucidate the (supraspinal) mechanisms responsible for the level of comfort, the sensation, that accompanies SCSwaveforms. As the MCAS can provide critical insight into cognitive-motivational processing of SCS, it could serve as a preclinical tool that allows for the optimization of supraspinal mechanisms of Burst SCS. It is important to note that the difference between Tonic SCS and Burst SCS, as observed for the exit latencies, was not present in the reflex-based Von Frey analysis. This suggests that the MCAS allows for assessment of behavioral changes in pain that are not detected by reflex-based testing. The importance of adding operant testing 
methods to the standard preclinical assessment battery for NPP has already been pinpointed by Vierck and colleagues [54]. In an extensive review they described numerous disparities between results for reflex-based testing and operant escape measures. Interestingly, the results of operant testing were often more consistent with the clinical setting, as compared to reflex-based measures. As NPP is a complex phenomenon, that is characterized by both sensory and affective disturbances [55], it is important that we now have a tool, the MCAS, which allows us to adequately assess supraspinal, cognitivemotivational aspects of NPP in a preclinical setting. A better understanding of the cognitivemotivational pain relieving aspects will allow further analysis and optimization of these specific characteristics of the Burst SCS-protocol.

Research question 5: Which brain areas are involved in the pain relieving effect of Burst SCS, as compared to Tonic SCS, in an animal model of chronic neuropathic pain?

In order to understand the supraspinal mechanisms and different aspects of pain relief induced with Burst SCS we used functional magnetic resonance imaging (fMRI) which allowed us to assess Blood-Oxygenation-Level Dependent (BOLD) signals in the brain of 17 chronic neuropathic (PSNL) rats. We specifically looked at brain regions associated with location and intensity of pain, as well as cognitive-motivational aspects of pain. The results can be summarized as follows: for both Tonic SCS and Burst SCS we observed an increase in BOLD-signal in brain regions associated with location and intensity of pain (somatosensory cortex and premotor cortex), and cognitive-emotional aspects of pain (amygdala, anterior cingulate cortex, insular cortex). However, Burst SCS induced a more profound BOLDincrease in brain areas overall, and brain areas associated with cognitive-emotional aspects of pain, as compared to Tonic SCS. The results from our study corroborate the prevailing hypothesis that Burst SCS stimulates pathways able to activate brain areas associated with cognitive-emotional aspects of pain $[6,14]$ and are in line with the behavioral outcome of our study described in Chapter 5. The cognitive-emotional brain areas are most likely activated by signals that travel along medial STT projection neurons in order to engage the cingulate cortex, the insula, and the amygdala. Significantly increased BOLD-signal was observed in the Amygdala, a key region of the medial STT-system, during Burst SCS, as compared to pre-stimulation and Tonic SCS. Furthermore, two additional key-regions of the medial pain network, the insula and the cingulate cortex, showed a much more profound increase in BOLD-signal during Burst SCS, as compared to Tonic SCS. This is in line with clinical work from the Ridder and colleagues who showed with encephalogram analysis that 
Burst SCS normalized the pain supporting/suppressing balance in contrast to Tonic SCS mode by a greater effect on the dorsal Anterior Cingulate Cortex (ACC) [14]. Nevertheless, it should also be noted that in our study Tonic SCS also increased BOLD-signal in areas associated with the medial spinothalamic pain pathway, although to a lesser extent than observed for Burst SCS. This suggests that the different stimulation paradigms, Burst and Tonic, are able to activate specific brain areas to a different extent. In the second chapter of this thesis, we discussed the behavioral effect of chronic neuropathic animals in response to various amplitudes of Burst SCS and Tonic SCS. From this, we concluded that a more complex relationship defines the interplay between the parameters of SCS and the therapeutic efficacy of SC, as the analgesic efficacy of Burst SCS showed a non-monotonic relation with Burst-amplitude. It seems that with the studies described in this thesis we have only just scratched the surface of parameter variations. More research into parameter variations for Burst SCS might reveal novel differences, yet also, commonalities, with existing and future waveforms. Moreover, it has been postulated that no one waveform will be optimal for all neuropathic pain patients, therefore the field might eventually move towards a more personalized waveform, 'tailor-made' for each patient. Yet, this requires a thorough understanding of the spinal and supraspinal mechanisms underlying (most) optimal pain relief. Extensive research has been performed, and it still being performed, in order to decipher the mechanisms responsible for the experience of pain. This has led to the formulation of solid frameworks such as the 'Pain Matrix' [56-59]. Future neuroimaging studies should aim to build a framework representative of the 'optimal pain relief signature'. This can then serve as a guideline for future SCS-waveform design. 


\section{Conclusion}

In summary, the results presented in this academic thesis show that a more complex relationship defines the interplay between parameters of Burst SCS, the charge delivered to the spinal cord, and the analgesic efficacy. Burst SCS exhibits a delayed anti-nociceptive effect after onset of stimulation, and a delayed washout of analgesia after stimulation is turned off, as compared with Tonic SCS. We also found, in a well-established chronic neuropathic animal model, that the analgesic effect of both Burst SCS and Tonic SCS is mediated via spinal GABAergic mechanisms. In a behavioral experiment, we were able to demonstrate that Burst-SCS specifically affects, much more than Tonic-SCS, the processing of cognitive-motivational aspects of pain. Lastly, we reported that both Tonic SCS and Burst SCS increase BOLD-signals in brain regions associated with cognitive-emotional aspects of pain. Yet, Burst SCS induced a more profound effect in these brain areas. Mechanism based insights and experimental studies as described in this thesis can lead to further optimization of the Burst-paradigm and eventually lead to better treatment of neuropathic pain in patients (CRPS/FBSS). 


\section{References}

1. Courtney, P., et al., Improved Pain Relief With Burst Spinal Cord Stimulation for Two Weeks in Patients Using Tonic Stimulation: Results From a Small Clinical Study. Neuromodulation, 2015. 18(5): p. 361-6.

2. Kriek, N., et al., Preferred frequencies and waveforms for spinal cord stimulation in patients with complex regional pain syndrome: A multicentre, double-blind, randomized and placebocontrolled crossover trial. Eur J Pain, 2017. 21(3): p. 507-519.

3. Miller, J.P., et al., Parameters of Spinal Cord Stimulation and Their Role in Electrical Charge Delivery: A Review. Neuromodulation, 2016. 19(4): p. 373-84.

4. Wille, F., et al., Altering Conventional to High Density Spinal Cord Stimulation: An Energy DoseResponse Relationship in Neuropathic Pain Therapy. Neuromodulation, 2017. 20(1): p. 71-80.

5. Temel, Y., et al., Monopolar versus bipolar high frequency stimulation in the rat subthalamic nucleus: differences in histological damage. Neurosci Lett, 2004. 367(1): p. 92-6.

6. De Ridder, D., et al., Burst spinal cord stimulation for limb and back pain. World Neurosurg, 2013. 80(5): p. 642-649 e1.

7. De Ridder, D., et al., Burst spinal cord stimulation: toward paresthesia-free pain suppression. Neurosurgery, 2010. 66(5): p. 986-90.

8. Meuwissen, K.P.V., et al., Conventional-SCS vs. Burst-SCS and the Behavioral Effect on Mechanical Hypersensitivity in a Rat Model of Chronic Neuropathic Pain: Effect of Amplitude. Neuromodulation, 2018. 21(1): p. 19-30.

9. Meuwissen, K.P.V., et al., Response to: Fundamental Differences in Burst Stimulation Waveform Design: Eliminating Confusion in the Marketplace. Neuromodulation, 2018. 21(7): p. 721-722.

10. Falowski, S.M., Fundamental Differences in Burst Stimulation Waveform Design: Eliminating Confusion in the Marketplace. Neuromodulation, 2018. 21(3): p. 320.

11. West DC, W.J., Strength-duration characteristics of myelinated and nonmyelinated bulbospinal axons in the cat spinal cord. J Physiol, 1983. ;337:37-50.

12. Abejon, D., et al., Is the introduction of another variable to the strength-duration curve necessary in neurostimulation? Neuromodulation, 2015. 18(3): p. 182-90; discussion 190.

13. Yearwood, T.L., et al., Pulse width programming in spinal cord stimulation: a clinical study. Pain Physician, 2010. 13(4): p. 321-35.

14. De Ridder, D. and S. Vanneste, Burst and Tonic Spinal Cord Stimulation: Different and Common Brain Mechanisms. Neuromodulation, 2016. 19(1): p. 47-59.

15. Smits, H., M. van Kleef, and E.A. Joosten, Spinal cord stimulation of dorsal columns in a rat model of neuropathic pain: evidence for a segmental spinal mechanism of pain relief. Pain, 2012. 153(1): p. 177-83.

16. Smits, H., et al., Experimental spinal cord stimulation and neuropathic pain: mechanism of action, technical aspects, and effectiveness. Pain Pract, 2013. 13(2): p. 154-68.

17. Moens, M., et al., Spinal cord stimulation modulates cerebral function: an fMRI study. Neuroradiology, 2012. 54(12): p. 1399-407.

18. Zhang, T.C., J.J. Janik, and W.M. Grill, Modeling effects of spinal cord stimulation on widedynamic range dorsal horn neurons: influence of stimulation frequency and GABAergic inhibition. J Neurophysiol, 2014. 112(3): p. 552-67.

19. Seltzer, Z., R. Dubner, and Y. Shir, A novel behavioral model of neuropathic pain disorders produced in rats by partial sciatic nerve injury. Pain, 1990. 43(2): p. 205-18.

20. Janssen, S.P., et al., Differential GABAergic disinhibition during the development of painful peripheral neuropathy. Neuroscience, 2011. 184: p. 183-94. 
21. Janssen, S.P., et al., Decreased intracellular GABA levels contribute to spinal cord stimulationinduced analgesia in rats suffering from painful peripheral neuropathy: the role of KCC2 and GABA(A) receptor-mediated inhibition. Neurochem Int, 2012. 60(1): p. 21-30.

22. Cui, J.G., B. Linderoth, and B.A. Meyerson, Effects of spinal cord stimulation on touch-evoked allodynia involve GABAergic mechanisms. An experimental study in the mononeuropathic rat. Pain, 1996. 66(2-3): p. 287-95.

23. Crosby, N.D., et al., Burst and Tonic Spinal Cord Stimulation Differentially Activate GABAergic Mechanisms to Attenuate Pain in a Rat Model of Cervical Radiculopathy. IEEE Trans Biomed Eng, 2015. 62(6): p. 1604-13.

24. Cui, J.G., et al., Effect of spinal cord stimulation on tactile hypersensitivity in mononeuropathic rats is potentiated by simultaneous $G A B A(B)$ and adenosine receptor activation. Neurosci Lett, 1998. 247(2-3): p. 183-6.

25. Linderoth, B., et al., Gamma-aminobutyric acid is released in the dorsal horn by electrical spinal cord stimulation: an in vivo microdialysis study in the rat. Neurosurgery, 1994. 34(3): p. 484-8; discussion 488-9.

26. Stiller, C.O., et al., Release of gamma-aminobutyric acid in the dorsal horn and suppression of tactile allodynia by spinal cord stimulation in mononeuropathic rats. Neurosurgery, 1996. 39(2): p. 367-74; discussion 374-5.

27. Cui, J.G., et al., Spinal cord stimulation attenuates augmented dorsal horn release of excitatory amino acids in mononeuropathy via a GABAergic mechanism. Pain, 1997. 73(1): p. 87-95.

28. Meuwissen, K.P.V., et al., Burst Spinal Cord Stimulation in Peripherally Injured Chronic Neuropathic Rats: A Delayed Effect. Pain Pract, 2018.

29. Barchini, J., et al., Spinal segmental and supraspinal mechanisms underlying the pain-relieving effects of spinal cord stimulation: an experimental study in a rat model of neuropathy. Neuroscience, 2012. 215: p. 196-208.

30. Song, Z., et al., Pain relief by spinal cord stimulation involves serotonergic mechanisms: an experimental study in a rat model of mononeuropathy. Pain, 2009. 147(1-3): p. 241-8.

31. Linderoth, B., et al., Dorsal column stimulation induces release of serotonin and substance $P$ in the cat dorsal horn. Neurosurgery, 1992. 31(2): p. 289-96; discussion 296-7.

32. Saade, N.E. and S.J. Jabbur, Nociceptive behavior in animal models for peripheral neuropathy: spinal and supraspinal mechanisms. Prog Neurobiol, 2008. 86(1): p. 22-47.

33. Foreman, R.D. and B. Linderoth, Neural mechanisms of spinal cord stimulation. Int Rev Neurobiol, 2012. 107: p. 87-119.

34. Linderoth, B. and R.D. Foreman, Physiology of spinal cord stimulation: review and update. Neuromodulation, 1999. 2(3): p. 150-64.

35. Holsheimer, J. and J.R. Buitenweg, Review: Bioelectrical mechanisms in spinal cord stimulation. Neuromodulation, 2015. 18(3): p. 161-70; discussion 170.

36. Holsheimer, J., Computer modelling of spinal cord stimulation and its contribution to therapeutic efficacy. Spinal Cord, 1998. 36(8): p. 531-40.

37. Snider, R.K., et al., Burst firing and modulation of functional connectivity in cat striate cortex. J Neurophysiol, 1998. 80(2): p. 730-44.

38. Truin, M., et al., Increased efficacy of early spinal cord stimulation in an animal model of neuropathic pain. Eur J Pain, 2011. 15(2): p. 111-7.

39. Truin, M., et al., Successful pain relief in non-responders to spinal cord stimulation: The combined use of ketamine and spinal cord stimulation. European Journal of Pain, 2011. 15(10).

40. Smits, H., et al., Effect of spinal cord stimulation in an animal model of neuropathic pain relates to degree of tactile "allodynia". Neuroscience, 2006. 143(2): p. 541-6.

41. Jaggi, A.S., V. Jain, and N. Singh, Animal models of neuropathic pain. Fundam Clin Pharmacol, 2011. 25(1): p. 1-28. 
42. Lind, G., et al., Baclofen-enhanced spinal cord stimulation and intrathecal baclofen alone for neuropathic pain: Long-term outcome of a pilot study. Eur J Pain, 2008. 12(1): p. 132-6.

43. Mogil, J.S., Animal models of pain: progress and challenges. Nat Rev Neurosci, 2009. 10(4): p. 283-94.

44. De Ridder, D., et al., A 2-center comparative study on tonic versus burst spinal cord stimulation: amount of responders and amount of pain suppression. Clin J Pain, 2015. 31(5): p. 433-7.

45. Vesper, J., et al., Burst SCS Microdosing Is as Efficacious as Standard Burst SCS in Treating Chronic Back and Leg Pain: Results From a Randomized Controlled Trial. Neuromodulation, 2018.

46. Deer, T., et al., Success Using Neuromodulation With BURST (SUNBURST) Study: Results From a Prospective, Randomized Controlled Trial Using a Novel Burst Waveform. Neuromodulation, 2018. 21(1): p. 56-66.

47. Gong, W.Y., L.M. Johanek, and K.A. Sluka, A Comparison of the Effects of Burst and Tonic Spinal Cord Stimulation on Hyperalgesia and Physical Activity in an Animal Model of Neuropathic Pain. Anesth Analg, 2016.

48. de Vos, C.C., et al., Burst spinal cord stimulation evaluated in patients with failed back surgery syndrome and painful diabetic neuropathy. Neuromodulation, 2014. 17(2): p. 152-9.

49. Demartini, L., et al., Comparison of Tonic vs. Burst Spinal Cord Stimulation During Trial Period. Neuromodulation, 2018.

50. Tjepkema-Cloostermans, M.C., et al., Effect of Burst Stimulation Evaluated in Patients Familiar With Spinal Cord Stimulation. Neuromodulation, 2016. 19(5): p. 492-7.

51. Kriek, N., et al., Preferred frequencies and waveforms for spinal cord stimulation in patients with complex regional pain syndrome: A multicentre, double-blind, randomized and placebocontrolled crossover trial. Eur J Pain, 2016.

52. Verrills, P., C. Sinclair, and A. Barnard, A review of spinal cord stimulation systems for chronic pain. J Pain Res, 2016. 9: p. 481-92.

53. Hou, S., K. Kemp, and M. Grabois, A Systematic Evaluation of Burst Spinal Cord Stimulation for Chronic Back and Limb Pain. Neuromodulation, 2016. 19(4): p. 398-405.

54. Vierck, C.J. and R.P. Yezierski, Comparison of operant escape and reflex tests of nociceptive sensitivity. Neurosci Biobehav Rev, 2015. 51: p. 223-42.

55. Torta, R., V. leraci, and F. Zizzi, A Review of the Emotional Aspects of Neuropathic Pain: From Comorbidity to Co-Pathogenesis. Pain Ther, 2017. 6(Suppl 1): p. 11-17.

56. Tracey, I. and P.W. Mantyh, The cerebral signature for pain perception and its modulation. Neuron, 2007. 55(3): p. 377-91.

57. Tracey, I. and E. Johns, The pain matrix: reloaded or reborn as we image tonic pain using arterial spin labelling. Pain, 2010. 148(3): p. 359-60.

58. Legrain, V., et al., The pain matrix reloaded: a salience detection system for the body. Prog Neurobiol, 2011. 93(1): p. 111-24.

59. Garcia-Larrea, L. and R. Peyron, Pain matrices and neuropathic pain matrices: a review. Pain, 2013. 154 Suppl 1: p. S29-43. 


\section{Valorization}

\section{Chapter 2}

The burst waveform has many parameters that can be explored and optimized, one such parameter being amplitude. Our research found that while both waveforms are equally effective, their optimal effects are achieved at different amplitude settings. To our surprise, the study received a number of critical comments; $d r$. De Ridder and dr. Vanneste criticized our study based on the shape of the waveform we used. Instead of a rebuttal, we would like to take this opportunity to underline the importance of objective and unbiased research, especially when it comes to assessing the various parameter variations of (burst) SCS. When objectivity is lost firm beliefs that lack substantial scientific foundation will prevail, causing further polarization, which will inevitably bring the progression of the field to a halt.

\section{Chapter 3}

Our investigation of the analgesic effect of burst and tonic SCS, over time, led to the observation that burst SCS requires significantly more time for its analgesic effect to fully wash in. The delayed wash-in, and subsequently delayed wash-out, had important consequences for the application of burst SCS in the clinical setting. For example, it is now corroborated by clinical observations that the burst waveform is not optimal for patients that require immediate pain relief. However, burst SCS simultaneously delivered with a waveform that does not exhibit a delayed wash-in (if the technology permits) could still yield successful outcomes in the aforementioned patient group.

\section{Chapter 4}

We demonstrated that GABA plays a key role in the analgesic mechanisms underlying burst SCS. In the past, similar experimental findings were successfully translated to the clinical setting; neuropathic pain patients with a deficient tonic SCS effect showed improved pain relief following intrathecal administration of subeffective doses of baclofen. Interestingly, our results were not in line with a study that investigated the GABAergic mechanisms underlying burst SCS in 2015. Taking into account the important role GABA plays in the mechanisms underlying tonic SCS we encourage further investigation of the GABAergic mechanisms underlying SCS waveforms. 


\section{Chapter 5}

We investigated a novel operant testing method able to assess motivational and cognitive aspects of pain processing. The pain field, especially the neuromodulation niche, is currently undergoing a shift of focus towards supraspinal processing. With the introduction of novel SCS-paradigms, that are hypothesized to have strong supraspinal effects, the preclinical field is in need of assessment methods able to address such hypotheses. In this chapter, we have paved the way for preclinical pain assessment able to address both the sensory and emotional aspects of pain. We believe that implementation of operant testing methods such as these can significantly improve the translation to the clinic.

\section{Chapter 6}

In the last chapter, by means of $\mathrm{fMRI}$, we assessed the supraspinal mechanisms of tonic and burst SCS in a chronic pain model. We found that burst SCS and tonic SCS both activate brain areas associated with the medial and lateral pain pathways. Additionally, we found that burst SCS might have additional analgesic properties over tonic SCS by the activation of brain areas associated with the reward-system and the raphe nuclei. In light of these new findings, we believe that more research is still required before we can advocate the use of specific waveforms for specific patient populations. 


\section{Curriculum Vitae}

Koen Meuwissen was born in Sittard, the Netherlands, on December the 8th in 1989. He obtained his high school degree (Atheneum) in 2009 at Trevianum Scholengroep in Sittard. In September 2009, he started his university education at Maastricht University where he enrolled in the Bachelor program of Psychology and Neuroscience. During his Bachelor internship, he studied the neural correlates of consciousness under the supervision of Dr. Tom de Graaf and Prof Dr. Alexander Sack at the department of Cognitive Psychology at Maastricht University. He obtained his Bachelor degree in 2012, in the same year he successfully applied for the Research master in Cognitive and Clinical Neuroscience with the specialization Fundamental Neuroscience. During his time in Maastricht, he joined the student association 'Koko' where he joined and chaired several committees. For his Master internship, he joined the research team of Prof. Dr. Laurence Lanfumey at the National Institute of Health and Medical Research (INSERM) in Paris, France. He was assigned to a project where he conducted the initial pharmacological profiling of synthetic compounds for cognitive enhancement of Donepezil in vivo. After successfully completing his Research Master studies in 2014, he started his PhD project at the department of Anesthesiology (MUMC+) under the supervision of Prof. Dr. Bert Joosten and Prof. Dr. Maarten van Kleef. For his PhD project, he studied the effects of Spinal Cord Stimulation (SCS) in experimental chronic neuropathic pain. He collaborated with the University of Utrecht, where he investigated the supraspinal mechanisms of the Burst SCS waveform by means of functional magnetic resonance imaging of brain activity in a rat model of chronic neuropathic pain. The results of his studies are presented in this thesis. During his PhD, he developed a great interest in neuromodulation and the medical device industry. Currently, he is working as a Field Clinical Specialist at Boston Scientific. 


\section{List of Publications}

2018 Meuwissen, K. P. V., Gu, J. W., Zhang, T. C., \& Joosten, E. A.

J. (2018). Conventional-SCS vs. Burst-SCS and the Behavioral Effect on Mechanical Hypersensitivity in a Rat Model of Chronic Neuropathic Pain: Effect of Amplitude. Neuromodulation, 21(1), 19-30. doi.org/10.1111/ner.12731.

Published.

2018 Meuwissen, K. P. V., Gu, J. W., Zhang, T. C., \& Joosten, E. A. J. (2018). Burst Spinal Cord Stimulation in Peripherally Injured Chronic Neuropathic Rats: A Delayed Effect. Pain Practice, 18(8), 988-996. doi.org/10.1111/papr.12701. Published.

2018 Meuwissen, K. P. V., Gu, J. W., Zhang, T. C., \& Joosten, E. A. J. (2018). Response to: Fundamental Differences in Burst Stimulation Waveform Design: Eliminating Confusion in the Marketplace. Neuromodulation, 21(7), 721722. doi.org/10.1111/ner.12857. Published.

2019 Meuwissen, K.P.V., van Beek, M., Hermes, D., Joosten, E.A.J. (2019). Mechanical Conflict-Avoidance System: Assessment of Motivational Aspects of Pain in Peripherally Injured Chronic Neuropathic Rats. Neuromodulation: Apr 11. doi: 10.1111/ner.12955. [Epub ahead of print].

2019 Meuwissen, K.P.V., de Vries, L.E., Zhang, T. C., Gu, J. W., Joosten, E.A.J. (2019). Burst and Tonic Spinal Cord Stimulation Both Activate Spinal GABAergic Mechanisms to Attenuate Pain in a Rat Model of Chronic Neuropathic Pain. Pain Practice: Accepted.

2019 Meuwissen, K.P.V., Van der Toorn, A., Joosten, E.A.J., Dijkhuizen, R. (2019). Burst Spinal Cord Stimulation in Peripherally Injured Chronic Neuropathic Rats: a functional magnetic resonance study. Pain: Submitted.

2017 van Reij, R. R. I., Meuwissen, K. P. V., \& Joosten, E. A. J. (2017). Anatomie en fysiologie van pijn. Anesthesiologie \& Intensive Care, 2017(4), 16-23. Published.

2015 Lardenoije, R., van den Hove, D. L. A., Vaessen, T. S. J., latrou, A., Meuwissen, K. P. V., van Hagen, B. T. J., ... Rutten, B. P. F. (2015). Epigenetic modifications in mouse cerebellar Purkinje cells: effects of aging, caloric restriction, and overexpression of superoxide dismutase 1 on 5-methylcytosine and 5hydroxymethylcytosine. Neurobiology of Aging, 36(11), 3079-3089. doi.org/10.1016/j.neurobiolaging.2015.08.001. Published. 


\section{ACKNOWLEDGEMENTS}

Allereerst mijn promotor en daily supervisor prof. Dr. Bert Joosten.

Vier jaar geleden begon ik aan mijn promotie-traject, met jou als dagelijkse begeleider. Al snel werd duidelijk dat jij een mooi team gerekruteerd had; een mix van Limburgse gezelligheid, Rotterdamse directheid en een alom vertegenwoordigde passie voor pijnonderzoek. Gedurende het project ben ik niet alleen gegroeid als onderzoeker maar ook als persoon, mede door de persoonlijke begeleiding en de positieve werksfeer. Tijdens het traject heb ik een passie voor neuromodulatie ontwikkeld. Daarom ben ik ervan overtuigd dat wij elkaar niet uit het oog zullen verliezen!

Mijn tweede promotor prof. Dr. Maarten van Kleef; ik heb het altijd zeer kunnen waarderen dat jij als afdelingshoofd anesthesiologie zo'n benaderbare en joviale houding had! Op de werkvloer heeft jouw wetenschappelijke input tijdens de onderzoeks-meetings mij vaak tot nieuwe inzichten gebracht.

Pap, ik kon altijd rekenen op jouw steun en advies. Jouw belangstelling voor mijn onderzoek heeft mij altijd veel motivatie gegeven. Het is dan ook aan jou om dit boekje samen te vatten voor de beide opa's ;)

Oma Tona, helaas heeft $u$ de verdediging niet meer mee mogen maken. Desalniettemin bestond er bij u geen enkele twijfel dat ik "doctor!" zou gaan worden en mocht/moest iedereen in het verpleegtehuis horen over mijn promotieonderzoek!

Mam en Anne, die waarschijnlijk dit dankwoord met aandacht zullen lezen;). Bedankt voor jullie steun en advies. Vanaf vandaag kunnen jullie naar dit boekje verwijzen wanneer iemand vraagt naar de kern van mijn onderzoek!

Marina, you are a tower of strength! Your unlimited supply of positivity and devotion have helped me through the seemingly endless days of writing ;) Now it is time to pick up the pace and continue to explore all that the other continents have to offer (and all that the southern part of our continent has to offer;).

Maarten en Roy; de fitboys! Wat waren wij een magisch trio bij UM-sport! In het begin stond ik sceptisch tegenover mijn lidmaatschap van de fitcrew....twee tot drie keer per week sporten....naast de dagelijkse werkzaamheden..... Maar al snel had ik de smaak te pakken. De inspanning tijdens de gymsessies gevolgd door de ontspanning erna hebben mij, zonder dat ik het meteen in de gaten had, de afgelopen 4 jaar erg geholpen! Al snel werd het lidmaatschap ook uitgebreid met extracurriculaire activiteiten zoals uitgaansavonden, 
festivals, Zweden en in de toekomst Canada! Heren, moge de vriendschap nog lang voortduren!

Vrienden, in Nederland, Europa, USA, in Maastricht, in Sittard en in Einighausen (ligt in Nederland). Een groot voordeel van het werken in een internationale setting is de wereldwijde vriendenkring die je eraan overhoudt. Echter, wanneer de wereld soms te groot wordt, is het ook fijn om weer 'back to the roots' een "boeren ambacht" feest bij te wonen in je eigen geboortedorp! Ik hoop dat de toekomst mij meer dan genoeg kansen zal geven om jullie allen van tijd tot tijd op te zoeken!

Het 'pijn team', te beginnen met Wiel, de man die je alles kon vragen! Ik denk niet dat de keren dat je mij uit de brand geholpen hebt op een hand te tellen zijn, zelfs nog nadat je met pensioen was! Glenn, de man die je alles kon vragen over horloges en boksen.... Maar enkel met die beschrijving zou ik jou te kort doen. Voordat wij beiden lid werden van het 'pijn-team' hadden we al een mooi avontuur achter de rug onder begeleiding van Carolin! Ik was dan ook aangenaam verrast toen Bert tijdens mijn interview vertelde dat ik veel zou gaan samenwerken met een persoon genaamd Glenn Franken; en of dat wel goed zou komen? Ik denk dat we 4 jaar later kunnen concluderen dat dat meer dan goed gekomen is!

Intussen is ons team behoorlijk gegroeid; Nynke, Roel, Perla, Rose, Lonne, Martijn en Thomas. Ook al was ik in de laatste fase van mijn traject niet altijd aanwezig bij de vele social events die jullie organiseerden, het deed me wel goed om te zien dat het 'pijn-team' met de dag gezelliger en hechter werd!

Jos, als coördinator van de researchmaster 'Fundamental Neuroscience' stond je aan de vooravond van mijn wetenschappelijke carrière. Gedurende de daaropvolgende twee jaren heb ik een leerzame maar ook avontuurlijke tijd beleefd met de door jou geselecteerde medestudenten! Al snel bleek dat jij jezelf onderscheidde van andere coördinatoren: je was betrokken! Betrokken bij onze vooruitgang, maar ook betrokken bij de festiviteiten;) Mede daarom vind ik het een eer dat jij ook de volgende stap in mijn wetenschappelijke carrière zal bijwonen, als voorzitter van de commissie.

Wendy Gu, you were my liaison to Boston Scientific. Your guidance and support were invaluable! It is safe to say that your friendly and open personality are among the top reasons as to why I joined Boston Scientific.

Tianhe Zhang and Rosana Esteller, I want to thank you for the outstanding and elaborate advice that I received from you during my PhD-trajectory. Your valuable comments, remarks and scientific consultation has contributed to the thesis that I am presenting here today. 
Sander van Kuijk, dank voor de statistische ondersteuning. Jouw talent om statistische zaken op een praktische en prettige manier te bespreken, heeft absoluut bijgedragen aan mijn traject.

Liong Liem, wij zullen elkaar ongetwijfeld nog tegenkomen in de neuromodulatie-wereld! $\mathrm{Na}$ onze fitness-gerelateerde discussies verwacht ik natuurlijk wel dat je dan minstens twee keer zo breed bent!

Daniel, jij zei het al in het eerste jaar van de master.... en nu zeg ik het: science is fun!

Jeroen and Bethany, our Friday evening dinners were unrivaled! The combination of wine, bad movies and deep discussions should be an internationally recognized recipe for mental relaxation.

Artemis, your outstanding (Greek) social skills have contributed to the overall atmosphere of the department! Your passion for research is inspiring, and your lust for life will have some adventures in store for the future, of that I am sure!

Robbert, you crazy bastard. Of course, I already knew you before I started my PhD. Yet, during my PhD our friendship developed through a number of great and memorable weekends in Paris and Maastricht! Even though I often lost a week due to recovery, I usually gained a month or more due to new motivation!

Simone, the United States is a better place now that you decided to move there with your family! Spread your knowledge and kindness in rural USA and I hope to see you soon!

Fred, in een ander leven waren we erkende horloge-connaisseurs geweest..... Echter, grote kunstenaars worden meestal pas na hun dood ontdekt!

Roy, bedankt voor de gastvrijheid aan de Charles Vos-Cour! En tevens bedankt voor de introductie tot ' $R$ ', je hebt uiteraard nog een Monster energy van me tegoed!

Nick en Britt, op de lab day out was het altijd even gezellig bij jullie in het down to earth hoekje!

Carolin, you were my supervisor during my first days at the lab! I could not have asked for a kinder and more welcoming supervisor! I was glad to see that you were still at the department when I returned to the lab as a PhD, and I always enjoyed your (vegetarian) contribution to the many social events that we both joined!

Jo and Yara. Jo, I am man enough to admit that I was a bit intimidated by you during my first days at the lab.... or when I wanted to leave the Alla before 6:00 am..... But what a great 
friend you turned out to be! Yara, you taught me that it is still possible to party while finishing your thesis! I took that advice to heart. I am confident that I will see you both in the future, starting with a ski-trip in Munich!

Ehsan and Gerben, I am glad you accompanied us to our very delightful trip to Porto! Besides that, we have shared some great moments at the many social events we all attended!

Sandra, een dame met doorzettingsvermogen! Ongeëvenaard op zowel werkgebied als sportief gebied;) Ik hoop dat je het naar je zin hebt in Aachen!

Paul, Fred, João en Maarten, I think it is safe to say we did not do anything out of the ordinary in Barcelona. We saw all the touristic attractions and learned a lot about the city's rich history!

What do you get when you combine an overwhelming level of positivity with unrivaled and meticulous climbing abilities: Gusta!

Richard, dank voor de altijd vriendelijke en persoonlijke ondersteuning vanuit de IVD. Gedurende mijn PhD heb ik ervaren dat je niet alleen een hardwerkend maar ook een bijzonder aardig persoon bent!

Voor alle mensen die ik vergeten ben: bij dezen bedankt voor de fantastische tijd die ik met jullie in Maastricht heb mogen beleven! 